Portland State University

PDXScholar

Spring 6-4-2015

\title{
Revealing Structural Organization with Liquid Crystal-based Spectral Imaging Polarimetry
}

James Campbell Gladish

Portland State University

Follow this and additional works at: https://pdxscholar.library.pdx.edu/open_access_etds

Part of the Other Electrical and Computer Engineering Commons

Let us know how access to this document benefits you.

\section{Recommended Citation}

Gladish, James Campbell, "Revealing Structural Organization with Liquid Crystal-based Spectral Imaging Polarimetry" (2015). Dissertations and Theses. Paper 2325.

https://doi.org/10.15760/etd.2322

This Dissertation is brought to you for free and open access. It has been accepted for inclusion in Dissertations and Theses by an authorized administrator of PDXScholar. Please contact us if we can make this document more accessible: pdxscholar@pdx.edu. 
Revealing Structural Organization with Liquid Crystal-based Spectral Imaging Polarimetry

by

James Campbell Gladish

A dissertation submitted in partial fulfillment of the requirements for the degree of

\author{
Doctor of Philosophy \\ in \\ Electrical and Computer Engineering
}

\author{
Dissertation Committee: \\ Donald Duncan, Chair \\ Scott Prahl \\ Martin Siderius \\ Lisa M. Zurk \\ Erik Sànchez
}

Portland State University

2015 
(C) 2015 James Campbell Gladish 


\begin{abstract}
Structural organization refers to the particular ordering of scatterers. Probing structural organization by imaging polarized spectral scatter provides insight into the composition of a medium, and can aid in remote sensing, the identification of tissue pathologies, and material characterization and differentiation. The vector nature of polarized light enables it to interact with optical anisotropies within a medium, while the spectral aspect of polarization is sensitive to small-scale structure. However, many polarization studies have limitations, as they provide qualitative image analysis, incomplete anisotropy information, or both. The ability to image the effects of anisotropy and small-scale structure at multiple wavelengths is key for parameterizing structural organization. The Stokes/Mueller formalism is a framework that quantifies a medium's complete spectral polarization response, and allows for the parameterization of structural organization. Additionally, advances in liquid crystal (LC) technology have resulted in new polarimetric devices. These computer-controlled devices impart spectral polarization effects on the millisecond timescale with no mechanically moving hardware, providing the ability for making rapid polarimetric measurements. This dissertation describes a methodology for revealing structural organization by exploiting the Stokes/Mueller formalism and by utilizing measurements from a spectral imaging polarimeter constructed from variable retardance LC devices, such as liquid crystal variable retarders (LCVRs) and a liquid crystal tunable filter (LCTF). The methodology includes developing the system, the Stokes/Mueller model, and all
\end{abstract}


of the procedures, calibrations, and data interpretation. Developing the system also consists of component and system calibration, a system sensitivity and performance analysis, and finally test measurements for system validation. The final validation measurement is made on a mineral sample for inferring structural organization. 


\section{Dedication}

In memory of my father, Jim Gladish. 


\section{Acknowledgments}

I want to express my deepest gratitude to my advisor, Dr. Donald Duncan, for the tremendous amount of support, guidance, encouragement, and insight he has given me over the years. He convincingly and persistently conveyed the virtue of rigorous scholarship, and instilled that in me as well. Without him this project would not have been possible.

I would also like to thank Drs. Scott Prahl, Martin Siderius, Lisa Zurk, and Erik Sànchez for serving on my committee. Their feedback and advice was instrumental in shaping this dissertation.

Additionally, I want to thank Dr. Alexander Ruzicka from the PSU Geology department for lending me samples and providing valuable input.

To my colleauges from both OHSU and PSU, I want to thank you for your helpful conversations and friendship. In particular, thanks to Niloy Choudhury, Elaine Wells-Gray, David Levitz, Kevin Phillips, Ravikant Samatham, John Gebbie, Sam Henry, Gabe Kniffin, Elizabeth Küsel, Lanfranco Muzi, and Scott Schecklman.

Last but certainly not least, thank you to my wonderful wife Jill for her love and enduring patience. Throughout this process, she has been my dearest friend, biggest cheerleader, voice of reason, and counselor. She was always there to cheer me up, and has stood by me through the good times and bad. 


\section{Table of Contents}

Abstract $\quad$ i

Dedication $\quad$ iii

Acknowledgments $\quad$ iv

List of Tables vii

List of Figures viii

1 Summary statement 1

1.1 Motivation . . . . . . . . . . . . . . . . . . 1

1.2 Contributions and significance . . . . . . . . . . . . 3

2 Research problem $\quad 5$

2.1 Introduction . . . . . . . . . . . . . . . . . 5

2.2 Structural organization . . . . . . . . . . . . . . . . . 8

2.3 Stokes/Mueller formalism . . . . . . . . . . . . . . . . . . 12

2.4 Polarized spectral scatter . . . . . . . . . . . . . . . . . 20

2.5 Polarimetry ........................ 23

2.5.1 Measurement strategies . . . . . . . . . . . . 26



2.6 Liquid crystal technology . . . . . . . . . . . . . . . . . 40

2.6.1 Liquid crystals . . . . . . . . . . . . . . . . . 41

2.6.2 Liquid crystal variable retarders . . . . . . . . . . . . . . . . 42

2.6.3 Liquid crystal tunable filters . . . . . . . . . . . . . . 46

3 System construction and assessment 49

3.1 Introduction . . . . . . . . . . . . . . . . . . . . 49

3.2 System configuration . . . . . . . . . . . . . . . 50

3.3 Component characterization ................. 53 
3.3.1 Transmittance measurements . . . . . . . . . . . . . 54

3.3.2 LCVR tuning curve calibration . . . . . . . . . . . 58

3.4 System assessment . . . . . . . . . . . . . . . . 65

3.4.1 Polarimeter calibration . . . . . . . . . . . . . . 65

3.4.2 LCVR alignment and temperature effects . . . . . . . . 73

3.4 .3 Performance analysis . . . . . . . . . . . . . . . . . . . . . 99

3.4.4 Test Measurements . . . . . . . . . . . . . . . . . . . 122

4 Coherence theory 147

4.1 Introduction . . . . . . . . . . . . . . . . . . . 147

4.2 The van Cittert-Zernike theorem . . . . . . . . . . . . 148

5 Structural organization measurements 158

5.1 Introduction . . . . . . . . . . . . . . . . . . 158

$5.2 \quad$ LCVR . . . . . . . . . . . . . . . . . . . . . . . 159

5.3 Terrestrial earth rock . . . . . . . . . . . . . . . . . . 185

5.4 Butterfly wing scales $\ldots \ldots \ldots \ldots \ldots \ldots$

5.5 Nanofibers . . . . . . . . . . . . . . . . . . . . . 204

6 Conclusion and future work 208

6.1 Conclusion . . . . . . . . . . . . . . . . . . . . . . . 208

6.2 Future work . . . . . . . . . . . . . . . . . . 210

6.3 Contributions . . . . . . . . . . . . . . . . . . . 212

$\begin{array}{ll}\text { References } & 215\end{array}$

Appendix A Relating the Jones and Stokes/Mueller formalisms $\quad 227$

Appendix B Common Jones and Mueller matrices 233

$\begin{array}{ll}\text { Appendix C Singular value decomposition } & 239\end{array}$

$\begin{array}{ll}\text { Appendix D Lyot filter } & 246\end{array}$

Appendix E Coherence functions 252

Appendix F Köhler illumination $\quad 262$

$\begin{array}{ll}\text { Appendix G Polarizer characterization } & 266\end{array}$

$\begin{array}{ll}\text { Appendix H Incidence angle effects } & 274\end{array}$ 


\section{List of Tables}

3.1 Training set retardance values (in waves) for the Stokes generator LCVRs. 73

3.2 Measurement retardance values (in waves) for the polarimeter LCVRs. 73

3.3 Nominal training set retardance values (in waves) . . . . . . . . . . . 103

3.4 Nominal Stokes generator retardance values (in waves). . . . . . . . . 103

3.5 Nominal retardance values (in waves) for the polarimeter measurements.103

3.6 High retardance values (in waves) for the training set. . . . . . . . . . 103

3.7 High retardance values (in waves) for the Stokes generator. . . . . . . 103

3.8 High retardance values (in waves) for the polarimeter measurements. 103

5.1 Description of the seven transmitted and scattered components associated with the LCTF and LCVR. . . . . . . . . . . . . . . . 171 


\section{List of Figures}

2.1 Right) Human eye with the cornea labeled; Left) Corneal thickness consisting primarily of the stroma; Top) alternating layers of collagen fibrils within the stroma. Figures taken from $[28-30]$. . . . . . . .

2.2 Optical effects produced by calcite, a birefringent mineral. When viewing a pencil through calcite, a double image is formed due to birefringence. Figure taken from $[31] \ldots \ldots \ldots \ldots \ldots$

2.3 Electric field vector orientations for the six polarization states that define the Stokes vector. . . . . . . . . . . . . . . . . . . . 14

2.4 Poincaré sphere showing the six polarization states that combine to produce the Stokes vector. . . . . . . . . . . . . . .

2.5 Schematic representation of a sequence of polarization elements with input and output Stokes vectors. . . . . . . . . . . . . 20

2.6 Schematic of the rotating polarizer measurement configuration. . . . . 27

2.7 Schematic of the rotating retarder measurement configuration. . . . . 28

2.8 Illustration of a Wollaston prism producing orthogonally polarized outputs exhibiting shear. . . . . . . . . . . . . 30

2.9 Nematic liquid crystal polarization devices. . . . . . . . . . . . 41

2.10 Meadowlark LCVR construction. Figure taken from [109]. Not to scale. 43

2.11 Schematic of the liquid crystal director and the fast and slow axes. The director is parallel to the long axis of the LCs (which is also the slow axis), and describes their preferred orientation. . . . . . . . . .

2.12 Schematic of the liquid crystal director and the liquid crystal tilt angle. The fast axis is parallel to the $x$-axis, which is perpendicular to the page. 46

2.13 Schematic of a single Lyot filter stage built using a liquid crystal element. Figure taken from $[112] \ldots \ldots \ldots$. . . . . . . 47

3.1 Schematic of the measurement configuration. The illumination module is on top and the detection module is on bottom. . . . . . . . . 51

3.2 Photograph of the complete measurement configuration. . . . . . . 52

3.3 Schematic of the component transmittance measurement. . . . . . . 55

3.4 Principal transmittances for the Newport polarizers. . . . . . . . . 57 
3.5 LCTF spectral transmittance. . . . . . . . . . . . . . . . 57

3.6 Spectral transmittance for the four LCVRs. . . . . . . . . . . . 58

3.7 Schematic of the calibration configuration for each LCVR. . . . . . . 59

3.8 Wrapped retardance tuning curve corresponding to equation (3.6) for LCVR 3 at $600 \mathrm{~nm} . \ldots \ldots \ldots$. . . . . . . . . . . . . 62

3.9 a) LCVR 1 tuning curves at three wavelengths (500,600, and $700 \mathrm{~nm}$ ),

b) LCVR 1 tuning curves as a function of wavlength and drive voltage. 63

3.10 a) LCVR 2 tuning curves at three wavelengths (500, 600, and $700 \mathrm{~nm})$,

b) LCVR 2 tuning curves as a function of wavlength and drive voltage. 63

3.11 a) LCVR 3 tuning curves at three wavelengths (500, 600, and $700 \mathrm{~nm}$ ),

b) LCVR 3 tuning curves as a function of wavlength and drive voltage. 64

3.12 a) LCVR 4 tuning curves at three wavelengths (500, 600, and $700 \mathrm{~nm}$ ),

b) LCVR 4 tuning curves as a function of wavlength and drive voltage. 64

3.13 Poincaré sphere showing the training set Stokes vectors. The red circles represent linear Stokes vectors, the blue stars represent primarily elliptical Stokes vectors. . . . . . . . . . . . . . . 72

3.14 Schematic of LCVR construction. Not to scale. . . . . . . . . . 76

3.15 Tilt and tip for the LCVRs. . . . . . . . . . . . . . . . 81

3.16 Schematic representation of the simulation configuration. . . . . . . 82

3.17 User-derived tuning curves for the four LCVRs. . . . . . . . . . 86

3.18 a) Comparison of an intentionally misaligned LCVR measurement and model prediction for LCVR 1. b) Residual between model predictions and measurements for a series of tilt angles. . . . . . . . . . .

3.19 a) Comparison of an LCVR measurement and model prediction for LCVR 1 at a given temperature. b) Residual between model predictions and measurements for a series of temperatures. . . . . . . . 88

3.20 Liquid crystal layer retardance sensitivities for LCVRs 1 and 2. . . . 89

3.21 Fast axis sensitivities for LCVRs 1 and $2 \ldots \ldots \ldots \ldots$. . . . . 90

3.22 Condition number as a function of wavelength for the misalignment configurations. Theoretical minimum is 1.732 , represented by the black line in each plot. . . . . . . . . . . . . . .

3.23 Mueller matrix element uncertainty at $400 \mathrm{~nm}$ (blue bars) and 720 $\mathrm{nm}$ (red bars) for the Stokes generator LCVR misalignments. The abscissa for each element represents the four Stokes generator LCVR misalignments $(1 \mathrm{X}, 1 \mathrm{Y}, 2 \mathrm{X}, 2 \mathrm{Y})$. The ordinate shows the standard deviation of the zero-mean error, which is unitless because the Mueller matrix is normalized. . . . . . . . . . . . . . . . . 
3.24 Mueller matrix element uncertainty at $400 \mathrm{~nm}$ (blue bars) and $720 \mathrm{~nm}$ (red bars) for the LCVR temperature perturbations. The abscissa of each element represents the four LCVRs in the system $(1,2,3,4)$. The ordinate shows the standard deviation of the zero-mean error, which is unitless because the Mueller matrix is normalized. . . . . . . . . . .

3.25 Simulation data illustrating the "stretching" effect of the LC layer retardance curve caused by misalignment.

3.26 a) Systematic retardance domain shaded in green on the Poincaré sphere. The red circles and blue stars denote the systematic training set. b) Retardance values for the systematic and arbitrary training sets. . . . . . . . . . . . . . . . . .

3.27 Mueller matrix uncertainty at $400 \mathrm{~nm}$ and $700 \mathrm{~nm}$ for low retardance values (i.e., the nominal values) for the Stokes generator and polarimeter for the Stokes generator misalignments. . . . . . . . . . . . . .

3.28 Mueller matrix uncertainty at $400 \mathrm{~nm}$ and $700 \mathrm{~nm}$ for the high retardance values for the Stokes generator and polarimeter for the Stokes generator misalignments. . . . . . . . . . . . . .

3.29 Mueller matrix uncertainty at $400 \mathrm{~nm}$ and $700 \mathrm{~nm}$ for low retardance values in the Stokes generator and high retardance values in the polarimeter for the Stokes generator misalignments. . . . . . . . . . .

3.30 Mueller matrix uncertainty at $400 \mathrm{~nm}$ and $700 \mathrm{~nm}$ for high retardance values in the Stokes generator and low retardance values in the polarimeter for the Stokes generator misalignments. . . . . . . . . .

3.31 Mueller matrix uncertainty at $400 \mathrm{~nm}$ and $700 \mathrm{~nm}$ for low retardance values (i.e., the nominal values) for the Stokes generator and polarimeter for the temperature perturbations. . . . . . . . . . . .

3.32 Mueller matrix uncertainty at $400 \mathrm{~nm}$ and $700 \mathrm{~nm}$ for the high retardance values for the Stokes generator and polarimeter for the temperature perturbations. . . . . . . . . . . . . . .

3.33 Mueller matrix uncertainty at $400 \mathrm{~nm}$ and $700 \mathrm{~nm}$ for low retardance values in the Stokes generator and high retardance values in the polarimeter for the temperature perturbations. . . . . . . . . . . .

3.34 Mueller matrix uncertainty at $400 \mathrm{~nm}$ and $700 \mathrm{~nm}$ for high retardance values in the Stokes generator and low retardance values in the polarimeter for the temperature perturbations. . . . . . . . . . . .

3.35 Mueller matrix uncertainty at $700 \mathrm{~nm}$ for low retardance values (i.e., the nominal values) for both the Stokes generator and polarimeter for the arbitrary and systematic training sets. . . . . . . . . . . . . 
3.36 Mueller matrix uncertainty at $700 \mathrm{~nm}$ for the high retardance values for both the Stokes generator and polarimeter for the arbitrary and systematic training sets. . . . . . . . . . . . . . . .

3.37 Mueller matrix uncertainty at $700 \mathrm{~nm}$ for low retardance values in the Stokes generator and high retardance values in the polarimeter for the arbitrary and systematic training sets. . . . . . . . . . . . .

3.38 Mueller matrix uncertainty at $700 \mathrm{~nm}$ for high retardance values in the Stokes generator and low retardance values in the polarimeter for the arbitrary and systematic training sets.

3.39 Mueller matrix uncertainty at $400 \mathrm{~nm}$ and $700 \mathrm{~nm}$ for low retardance values (i.e., the nominal values) for both the Stokes generator and polarimeter for a 6 -member systematic training set. . . . . . . . .

3.40 Mueller matrix uncertainty at $400 \mathrm{~nm}$ and $700 \mathrm{~nm}$ for low retardance values (i.e., the nominal values) for both the Stokes generator and polarimeter for a 12-member systematic training set. . . . . . . . . .

3.41 Mueller matrix uncertainty at $400 \mathrm{~nm}$ and $700 \mathrm{~nm}$ for low retardance values (i.e., the nominal values) for both the Stokes generator and polarimeter for a 24-member systematic training set. . . . . . . . .

3.42 Mueller matrix uncertainty at $400 \mathrm{~nm}$ and $700 \mathrm{~nm}$ for low retardance values (i.e., the nominal values) for both the Stokes generator and polarimeter for a 36-member systematic training set.

3.43 Mueller matrix uncertainty at $400 \mathrm{~nm}$ and $700 \mathrm{~nm}$ for low retardance values (i.e., the nominal values) for both the Stokes generator and polarimeter for a 48-member systematic training set. . . . . . . . .

3.44 a) Image of the $10 \times 10$ micrometer grid on the distortion target. b) Image of groups six and seven on the resolution target. The colored boxes contain element six of group seven, which has the maximum target resolution of $228 \mathrm{lp} / \mathrm{mm}$ in object space. . . . . . . . . . . . .

3.45 Cropped and zoomed images of the colored boxes from Fig. 3.44b, a) Vertical lines from the green box, b) Horizontal lines from the yellow

3.46 Contrast as a function of spatial frequency for the line patterns. . . . 126

3.47 Schematic (plan view) of the measurement configuration for measuring specular reflection from the mirror. . . . . . . . . . . . . . . 127

3.48 Condition number images for the two configurations at two wavelengths. 128

3.49 Condition number mean and standard deviation as a function of wavelength for the two configurations. . . . . . . . . . . . . . . . . . 128

3.50 Air Mueller matrix mean and standard deviation as a function of wavelength for the two configurations. . . . . . . . . . . . . . 
3.51 RMS error for the entire air Mueller matrix as a function of wavelength for the two configurations. . . . . . . . . . . . . . .

3.52 Condition number mean and standard deviation as a function of wavelength for the five training set sizes. The data are separated into two wavelength regions for clearer interpretation. . . . . . . . . . .

3.53 Air Mueller matrix mean and standard deviation as a function of wavelength for the five training set sizes. . . . . . . . . . . .

3.54 RMS error for the entire air Mueller matrix as a function of wavelength for the five training set sizes. . . . . . . . . . . . .

3.55 Mean and standard deviation of each polarizer Mueller matrix image as a function of wavelength. The red dashed lines represent the error bounds from the uncertainty expressed by equation (3.29). . . . . . .

3.56 Mean and standard deviation of each HWP Mueller matrix image as a function of wavelength for a fast axis orientation of $\approx 0^{\circ}$. The red dashed lines represent the error bounds from the uncertainty expressed by equation $(3.29) . \ldots \ldots \ldots \ldots \ldots \ldots$

3.57 Mean and standard deviation of each HWP Mueller matrix image as a function of wavelength for a fast axis orientation of $\approx 30^{\circ}$. The red dashed lines represent the error bounds from the uncertainty expressed by equation $(3.29) . \ldots \ldots \ldots \ldots \ldots \ldots$

3.58 Mean and standard deviation of each HWP Mueller matrix image as a function of wavelength for a fast axis orientation of $\approx 60^{\circ}$. The red dashed lines represent the error bounds from the uncertainty expressed by equation (3.29). . . . . . . . . . . . . . . . .

3.59 Mean and standard deviation of each QWP Mueller matrix image as a function of wavelength for a fast axis orientation of $\approx 0^{\circ}$. The red dashed lines represent the error bounds from the uncertainty expressed by equation (3.29). . . . . . . . . . . . . . . . . .

3.60 Mean and standard deviation of each QWP Mueller matrix image as a function of wavelength for a fast axis orientation of $\approx 30^{\circ}$. The red dashed lines represent the error bounds from the uncertainty expressed by equation (3.29). . . . . . . . . . . . . . . . .

3.61 Mean and standard deviation of each QWP Mueller matrix image as a function of wavelength for a fast axis orientation of $\approx 60^{\circ}$. The red dashed lines represent the error bounds from the uncertainty expressed by equation $(3.29) . \ldots \ldots \ldots \ldots \ldots \ldots \ldots$

3.62 Specific rotation as a function of wavelength for glucose (ORD curve). 140

3.63 Glucose measurements represented by a) equation (3.31), b) equation (3.33) 
3.64 Glucose measurement error bounds for the a) regular optical activity computation, b) modified optical activity computation. . . . . . . 143

4.1 Schematic for illustrating the propagation of mutual coherence. . . . . 150

4.2 Geometry describing the van Cittert-Zernike theorem. . . . . . . . . . 152

5.1 Schematic of the LCVR construction. Not to scale. . . . . . . . . 160

5.2 User-derived tuning curves for the two LCVRs used. . . . . . . . . . . 162

5.3 Schematic of the LCVR spectral scatter measurements. . . . . . . . . 168

5.4 Illustration of the seven transmitted and scattered components associated with the LCTF and LCVR. . . . . . . . . . . . . . . 171

5.5 Basic schematics of the four measurement configurations for isolating LCVR scatter. In parentheses are the numbers corresponding to the measured components for each configuration. . . . . . . . . . . .

5.6 Fractional scattering component $\log _{10}\left(F_{R}(V, \lambda)-F_{R}(10, \lambda)\right)$ as a function of drive voltage and wavelength for the two LCVRs at the three fast axis orientations. This figure shows how the scattered intensity varies with drive voltage, wavelength, and fast axis orientation, and also the repeatability of the measurements. . . . . . . . . . . . .

5.7 Four individual scattering spectra at various drive voltages and their respective model fits to Eq. 5.6 for LCVR 2 at a fast axis of $90^{\circ}$. This figure qualitatively shows the accuracy with which the model represents the measurement. . . . . . . . . . . . . . . . . . . 176

5.8 Liquid crystal tilt angle as a function of drive voltage. . . . . . . . . 177

5.9 Correlation length $L$ as a function of tilt angle for the two LCVRs at the three fast axis orientations. . . . . . . . . . . . 177

5.10 The power exponent $\alpha$ as a function of tilt angle for the two LCVRs at the three fast axis orientations . . . . . . . . . . .

5.11 Brightfield microscope image of the terrestrial earth rock. This image did not come with a scale bar, but the sample is $\approx 8 \mathrm{~mm}$ wide, $\approx 28$ $\mathrm{mm}$ long, and has a thickness of $\approx 36 \mu \mathrm{m}$. The green numbered boxes correspond to regions imaged by the polarimeter.

5.12 Mueller matrix images for region 1 at two wavelengths. The $(1,1)$ element is the raw intensity image with a separate colorbar. . . . . .

5.13 Model fits to the $(4,3)$ element for a single point from region 1 . The fitted parameters were then applied to the analytic forms of the remaining Mueller matrix elements. . . . . . . . . . . . . . .

5.14 Results from region 1. Raw intensity image (top left) and SEM-EDS image (top right), along with results from the model fits to the Mueller matrix element $(4,3)$ (bottom three images) . . . . . . . . . . 
5.15 Results from region 2. Raw intensity image (top left) and SEM-EDS image (top right), along with results from the model fits to the Mueller matrix element $(4,3)$ (bottom three images). . . . . . . . . . . . .

5.16 Results from region 3. Raw intensity image (top left) and SEM-EDS image (top right), along with results from the model fits to the Mueller matrix element $(4,3)$ (bottom three images). . . . . . . . . . . .

5.17 Results from region 4. Raw intensity image (top left) and SEM-EDS image (top right), along with results from the model fits to the Mueller matrix element $(4,3)$ (bottom three images). . . . . . . . . . . . .

5.18 Results from region 5. Raw intensity image (top left) and SEM-EDS image (top right), along with results from the model fit to the Mueller matrix element $(3,3)$ (bottom three images). . . . . . . . . . . . . 195

5.19 Raw intensity backscatter image of the butterfly wing scales. . . . . . 201

5.20 Mueller matrix backscatter images from the butterfly wing scales at two wavelengths. The $(1,1)$ element is the raw intensity image with a separate colorbar. . . . . . . . . . . . . . . . 202

5.21 Raw intensity backscatter image of the nanofiber mat. . . . . . . . . 205

5.22 Mueller matrix backscatter images from the nanofibers at two wavelengths. The $(1,1)$ element is the raw intensity image with a separate colorbar. . . . . . . . . . . . . . . 206 


\section{Summary statement}

\subsection{Motivation}

This work aims to use the properties of light, such as intensity, wavelength, and polarization, to infer the properties of a medium. More specifically, this work describes a methodology for revealing a medium's structural organization, and consists of a thorough description of the conceptual framework, the measurement system, and the interpretation of measurements. The conceptual framework describes a formalism for mathematically manipulating polarized light, which is used for system calibration and for simulating polarization measurements. In addition, the framework describes the relationship between the aforementioned properties of light and structural organization. Details regarding the measurement system, a liquid crystal-based spectral imaging goniometric polarimeter, include a description of its components, construction, and calibration. Lastly, measurement interpretation involves determining

the significance of the data, and how it can used for inferring structural organization.

The vector nature of polarized light enables it to interact with two different 
axes within a medium. If optical anisotropies are present along these axes, the resultant vector changes. In addition, the spectral aspect of polarization is sensitive to small-scale structure, which can produce a resonance phenomenon that results in a periodicity in the spectral polarization signature. Thus, the study of structural organization through spectral imaging polarimetry is sensitive to multiple effects. Furthermore, the field of polarimetry has seen significant growth in recent years, thanks in part to advances in liquid crystal (LC) technology. These advances have produced new devices, such as the liquid crystal variable retarder (LCVR) and the liquid crystal tunable filter (LCTF). This research utilizes the fundamentals of polarimetry and the recent advances in polarimetric technology to develop a new polarimetric approach for studying structural organization. The rationale for this approach is:

1. Many polarization studies have limitations, as they provide qualitative image analysis, incomplete anisotropy information, or both. The ability to image the effects of anisotropy and small-scale structure at multiple wavelengths enhances the assessment of structural organization, as it allows for a more comprehensive evaluation of the medium. This work uses the Stokes/Mueller formalism for describing a medium's complete spectral polarization response, which incorporates optical anisotropies such as retardance. Using this formalism, structural organization parameters can be inferred.

2. LC-based spectral polarization devices feature millisecond switching times, no mechanically moving hardware, a wide range of tunable states, and electronic 
control. These attributes allow for an approach that avoids error typically associated with mechanical movement. Additionally, other methods can be time consuming and error-prone as they employ rotating filter wheels, polarization elements that undergo rotation or translation, or polarization elements that require movement into and out of the system. LC-based spectral imaging polarimetry provides a rapid, automated approach for obtaining spatially resolved estimates of the complete spectral Mueller matrix.

\subsection{Contributions and significance}

This work will enhance the polarimetry body of knowledge by making several contributions to this area of study. These contributions will be to:

1. Develop a methodology for revealing structural organization with spectral polarimetric imaging. This methodology consists of system construction and measurement interpretation. No polarimetric system utilizes the combination of four LCVRs with an LCTF to generate polarization states and analyze spectral

polarization effects. The current system is capable of making spatial, spectral, polarization, and scatter measurements. Spectral polarization measurements will be used for inferring structural organization parameters of minerals in rock samples. These parameters will be in the context of the Stokes/Mueller calculus.

2. Develop a new error analysis technique for LCVR-based polarimetry. LCVR 
misalignment and temperature effects cause systematic error, which propagates through to the sample Mueller matrix. Simulations show a relationship exists between LCVR misalignments and temperature perturbations, and error in specific Mueller matrix elements.

3. Characterize LCVRs in terms of scatter and spatial organization. The nanometer size of the liquid crystals inside LCVRs suggest these devices produce Rayleigh scatter. However, the liquid crystal spatial organization produces non-Rayleigh scatter. These effects are dictated by the state of the LCVR, where the effects also impact system performance. Thus, LCVR characterization serves as a component of system performance analysis, and as a test case for interpreting structural organization measurements.

4. System optimization of LCVR-based polarimeters. The simulation architecture from the error analysis technique can also be used for exploring the optimum number and type of training set Stokes vectors and the optimum polarimeter settings. Optimization ultimately minimizes calibration time and increases accuracy. 


\section{Research problem}

\section{$2.1 \quad$ Introduction}

The motivation for this work is to infer the properties of a medium from the properties of light, such as intensity, wavelength, and polarization. In this work, a methodology is described for revealing structural organization, where this methodology uses concepts from the Stokes/Mueller calculus and measurements from a liquid crystal-based spectral imaging goniometric polarimeter.

Structural organization refers to the particular ordering of scatterers. Measuring spectral polarization effects at various observation angles (i.e., goniometry) serves as a method for probing structural organization. These effects can reveal structural organization features that describe the scatterers in a medium, such as shape, organization, and orientation [1]. For example, spectral features aid in distinguishing between media types [2] or medium constituents [3]. Polarization effects reveal characteristics about the structure and the symmetry of the scatterers [4-7]. Angular information discloses structural information about the scatterers [8,9]. Therefore, probing structural organization provides insight into the composition of a medium, 
and can aid in identifying tissue pathologies [10-13], remote sensing [2, 14, 15], and material characterization and differentiation [16-18].

Another aspect of structural organization includes directionally-dependent properties, or anisotropy. Thus, if scatterers have a particular ordering that is consistent in a given direction, it will exhibit anisotropy. These effects can be probed with polarimetry, as the vector nature of polarized light is sensitive to directionallydependent properties. A polarimetric formalism for describing the polarization-altering properties of a medium is the Stokes/Mueller calculus [19]. This formalism parameterizes optical anisotropies in terms of retardance.

Structural organization can also be probed by the spectral nature of polarized light. For example, when small-scale structure exists in a medium, it can produce a resonance phenomenon that is evident in the spectral polarization signature. The resonance causes a periodicity in the polarization response, and this periodicity is related to the structure [4].

Another formalism for parameterizing structural organization comes from the field of coherence theory, and is the generalized van-Cittert Zernike theorem. This theorem is a scalar representation of light propagation that relates the far field intensity pattern to the source coherence [20]. Although this theorem does not account for polarization effects, a method is described that uses this theorem while still incorporating polarized light. 
Typical methods for measuring polarized spectral scatter can be cumbersome, as moving elements (i.e., rotating filter wheels and polarizers or translating prisms) can be time consuming and error-prone, and point detectors do not provide spatial information. Implementing spectral polarization measurements has recently been improved upon through developments in liquid crystal (LC) technology [21]. Advances in liquid crystal technology have resulted in new LC-based spectral polarization devices. These computer-controlled devices impart spectral polarization effects on the millisecond timescale with no mechanically moving hardware, providing the ability for making rapid spectral polarimetric measurements.

This dissertation describes a method for elucidating structural organization by exploiting the Stokes/Mueller calculus and by utilizing a measurement system constructed from variable retardance $\mathrm{LC}$ devices, such as liquid crystal variable retarders (LCVRs) and a liquid crystal tunable filter (LCTF). Additionally, this dissertation will describe structural organization and the aforementioned theories in detail, while also providing a comprehensive analysis of the system and its individual components. The analysis will include individual component characterization, a description of the system construction, calibration, and optimization, and a new error analysis technique for liquid crystal-based active polarimetry. Upon system calibration and optimization, polarization measurements are made on several known samples. Finally, the system is used for parameterizing the structural organization of minerals in a rock sample. 
The remainder of this dissertation explains the research problem, liquid crystal devices, relevant models, measurement system, and results. Chapter 2 provides information describing the research problem, such as structural organization, the Stokes/Mueller formalism, polarimetry measurement strategies and optimization, and liquid crystal technology. Chapter 3 describes system construction and calibration, which consists of component and system calibration, a sensitivity and performance analysis, and test measurements. Chapter 4 provides details about coherence theory and the van Cittert-Zernike theorem. Chapter 5 shows results from structural organization measurements. Chapter 6 includes conclusions, future work, and a list of publications relevant to this work. Finally, the appendices contain details supporting specific sections of the dissertation.

\subsection{Structural organization}

Structural organization is a fundamental property of any medium, whether the medium is natural or man-made. The ability to quantify structural organization is very important in understanding the medium as a whole and how the medium interacts with its environment. This section provides a description of structural organization, presents several examples, and describes various optical effects caused by structural organization.

In the current context, structural organization refers to the specific ordering 
of subwavelength scatterers, where the ordered scatterers produce optical effects that are different from the effects generated by the individual scatterers themselves. As a result, the optical effects are due to morphology (i.e., the form and structure of specific features) rather than molecular makeup [22,23]. Optical effects typically include modifications to the scattered intensity as a function of wavelength, polarization, and propagation direction. These angle-dependent color and angle-dependent polarization effects are the result of light interacting with subwavelength scatterers through interference, diffraction, or scattering [22,23].

Many biological and industrial examples exhibit specific optical effects that depend on structural organization. For instance, structural color in nature can be found in insects (beetles, butterflies, and moths), birds (hummingbirds and peacocks), and gemstones (opal and pearl) $[22,23]$. These examples have subwavelength structures that provide precise control over optical characteristics such as hue, brightness, saturation, and directionality [24]. The human cornea also controls directionality, as the specific organization of the nanometer-sized collagen fibrils cause transparency in the cornea [25]. In terms of polarization, certain beetles [22] and butterflies [26] reflect specific circular and linear polarization states, respectively, based on their structural organizations, rather than selective molecular absorption. Minerals are another example of media that can alter polarization. Because of their unique crystalline structure, minerals exhibit different refractive indices along different axes within the medium, called birefringence [19]. 




Figure 2.1: Right) Human eye with the cornea labeled; Left) Corneal thickness consisting primarily of the stroma; Top) alternating layers of collagen fibrils within the stroma. Figures taken from [28-30]

Two examples from above, the cornea and minerals, warrant additional discussion. The cornea is the transparent outer window of the eye. In the center of the eye, the cornea is $\approx 500 \mu \mathrm{m}$ thick (see Fig. 2.1), with most of that dedicated to the stroma [27]. Stromal thickness consists of over 200 layers of parallel collagen fibrils, where the layers are arranged at various rotation angles throughout the depth of the stroma [27]. As an example, the top of Fig. 2.1 shows three stromal layers, where the fibrils in the middle layer are oriented such that they are perpendicular to the plane of the paper. Collagen fibrils have diameters of $\approx 30 \mathrm{~nm}$, and scattering predictions based on this size show the cornea should be opaque [25]. However, the close-packing of the fibrils (i.e., their structural organization) cause the cornea to be transparent [25].

Minerals also produce specific optical effects. Because of their crystalline structure, minerals have different refractive indices along their crystallographic axes. The 




Figure 2.2: Optical effects produced by calcite, a birefringent mineral. When viewing a pencil through calcite, a double image is formed due to birefringence. Figure taken from [31].

relative difference between the refractive indices is called birefringence. When light is incident on a birefringent mineral, the refractive index mismatch refracts the light into two beams, which travel at different velocities. Thus, when viewing a scene through a birefringent mineral such as calcite, a double image is seen due to the birefringence. Figure 2.2 shows a pencil when viewed through calcite [31].

The biological examples cited above have also motivated industrial applications. The natural photonic structures of insects have been the template for photonic crystals in integrated optics technologies $[32,33]$. By mimicking natural structures, photonic crystals have been developed for applications in detectors, spectroscopes, display screens, and integrated circuits [32]. Additionally, the polarization-altering helicoidal structure in some beetle shells have inspired liquid crystal analogues and optical diodes [34]. Finally, other more common industrial applications that produce angle-dependent color or angle-dependent polarization effects based on structural organization exist. These include special-effect automotive paints and security 
printing [35], diffraction gratings [36], antireflection surfaces [37], and thin-film polarizers [38].

As can be seen from this section, typical optical effects caused by structural organization involve the wavelength, polarization, and propagation direction of light. In terms of corneal characterization, interference effects must be accounted for, thus a statistical characterization involving fields is more appropriate. On the other hand, for mineral characterization, a formalism is needed that uses intensity and can account for polarization effects. The Stokes/Mueller formalism does this, as it uses intensities for describing polarization effects as a function of wavelength and propagation direction, and is the topic of the next section.

\subsection{Stokes/Mueller formalism}

The Stokes/Mueller formalism fully describes the polarization state of light and the polarization-altering properties of a medium in terms of the Stokes vector and the Mueller matrix, respectively. These two constructs can also be a function of wavelength, observation angle, and spatial coordinates (e.g., an image). This formalism assumes the incoherent addition of waves, and can account for any polarization state and depolarizing systems. Additionally, it does so with a real observable quantity, intensity [39]. Given that the Stokes/Mueller formalism incorporates an easily 
measurable quantity, intensity, it will be used instead of another commonly used formalism, the Jones formalism. For additional information about the Jones formalism and how it relates to the Stokes/Mueller formalism, see Appendix A.

\section{The Stokes vector}

The Stokes vector describes the polarization content of an incident beam. Defining the Stokes vector begins with a description of polarized light, as fundamental polarization states constitute a Stokes vector. The polarization state of light refers to the direction of oscillation of the electric field vector, which can be resolved into two orthogonal components, $E_{x}$ and $E_{y}[40]$. Depending on the polarization state, either one or both of these components exist, often with a constant relative phase delay $\delta$ between them. These three values define the polarization state.

Six polarization states are used for defining the Stokes vector, each with specific values for $E_{x}, E_{y}$, and $\delta[40]$. For example, when $E_{x}=1, E_{y}=0$, and $\delta=0$ radians, horizontal linear polarization results (see Fig. 2.3a). Values of $E_{x}=0, E_{y}=1$, and $\delta=0$ radians produce vertical linear polarization, shown in Fig. 2.3b. The combination of $E_{x}=E_{y}=1$ and $\delta=0$ radians yields $+45^{\circ}$ linear polarization (Fig. 2.3c), while $E_{x}=E_{y}=1$ and $\delta=\pi$ radians gives $-45^{\circ}$ linear polarization (Fig.2.3d). Finally, for right-circular polarization (RCP), $E_{x}=E_{y}=1$ and $\delta=\pi / 2$ radians (Fig. 2.3e), and for left-circular polarization (LCP), $E_{x}=E_{y}=1$ and $\delta=3 \pi / 2$ radians (Fig. 2.3f). RCP and LCP obtain their names from the path the tip of the electric 




(a) Horizontal linear polarization



(c) $+45^{\circ}$ linear polarization



(e) Right-circular polarization (RCP)



(b) Vertical linear polarization



(d) $-45^{\circ}$ linear polarization



(f) Left-circular polarization (LCP)

Figure 2.3: Electric field vector orientations for the six polarization states that define the Stokes vector. 
field vector traces in time. In other words, when observing RCP by looking towards the source (i.e., in the $-z$ direction in Fig. 2.3e), the tip traces a circular path in a clockwise direction. Conversely, when observing LCP by looking towards the source, the tip traces a circular path in a counterclockwise direction.

A Stokes vector can describe any polarization state of light, either unpolarized, partially polarized, or fully polarized. The Stokes vector $\mathbf{S}$ is given by $[40,41]$

$$
\mathbf{S}=\left[\begin{array}{c}
I \\
Q \\
U \\
V
\end{array}\right]=\left[\begin{array}{c}
E_{x}^{2}+E_{y}^{2} \\
E_{x}^{2}-E_{y}^{2} \\
2 E_{x} E_{y} \cos \delta \\
2 E_{x} E_{y} \sin \delta
\end{array}\right]=\left[\begin{array}{c}
I_{H}+I_{V} \\
I_{H}-I_{V} \\
I_{+45^{\circ}}-I_{-45^{\circ}} \\
I_{R C P}-I_{L C P}
\end{array}\right]
$$

where $I$ is the total intensity represented by the sum of the horizontally polarized component $I_{H}$ and the vertically polarized component $I_{V}, Q$ describes the amount of linear horizontal or vertical polarization and is the difference between $I_{H}$ and $I_{V}$, $U$ describes the amount of linear $\pm 45^{\circ}$ polarization and is the difference between the polarized components $I_{+45^{\circ}}$ and $I_{-45^{\circ}}$, and $V$ describes the amount of right- and left-circular polarization and is the difference between $I_{R C P}$ and $I_{L C P}$. Additionally, for fully polarized light, the Stokes vector is normalized relative to the total intensity of the beam. Thus, the $I$ element is equal to one, and the other three elements $(Q$, $U$, and $V$ ) have values on the interval $[-1,1]$. 
Partially polarized light is a combination of unpolarized light and fully polarized light. Partial polarization is expressed as

$$
\mathbf{S}_{\text {partial }}=\mathbf{S}_{\text {unpolarized }}+\mathbf{S}_{\text {polarized }}=(1-D O P)\left[\begin{array}{c}
I \\
0 \\
0 \\
0
\end{array}\right]+D O P\left[\begin{array}{c}
I \\
Q \\
U \\
V
\end{array}\right] \text {, }
$$

where DOP refers to the degree of polarization. The DOP describes the degree to which the light is polarized, and is defined as

$$
D O P=\frac{\sqrt{Q^{2}+U^{2}+V^{2}}}{I},
$$

with values on the interval $[0,1]$. The Stokes vector elements can also be combined to produce other metrics that describe the degree to which the light is polarized. These include the degree of linear polarization (DOLP) with values on the interval $[0,1]$, and the degree of circular polarization (DOCP) with values on $[-1,1][19]$,

$$
\begin{gathered}
D O L P=\frac{\sqrt{Q^{2}+U^{2}}}{I}, \\
D O C P=\frac{V}{I} .
\end{gathered}
$$

It is often convenient to visualize different states of polarized light relative 




Figure 2.4: Poincaré sphere showing the six polarization states that combine to produce the Stokes vector.

to each other. The Poincaré sphere is a graphical tool that aids in this (see Fig. 2.4), where the three sphere axes correspond to the elements $Q, U$, and $V$ of the Stokes vector. This graphical tool is a unit sphere where completely polarized light is represented on its surface by a point, with coordinates specified by the Stokes vector elements. Linear polarization states lie on the equator, while the north and south poles represent right- and left-circular polarization, respectively. Elliptical polarization states lie in between the equator and the poles [39]. Figure 2.4 shows the locations of the six polarization states of the Stokes vector on the Poincaré sphere.

\section{The Mueller matrix}

The Mueller matrix $\mathbf{M}$ is a $4 \times 4$ real matrix describing the polarization-altering properties of a medium or optical component (for examples of component Mueller 
matrices, see Appendix B: Common Mueller matrices). Given an incident Stokes vector $\mathbf{S}$, the Mueller matrix describes how a medium linearly transforms the incident Stokes vector to an output Stokes vector $\mathbf{S}^{\prime}$

$$
\mathbf{S}^{\prime}=\mathbf{M S}=\left[\begin{array}{llll}
m_{11} & m_{12} & m_{13} & m_{14} \\
m_{21} & m_{22} & m_{23} & m_{24} \\
m_{31} & m_{32} & m_{33} & m_{34} \\
m_{41} & m_{42} & m_{43} & m_{44}
\end{array}\right] \mathbf{S} .
$$

Although not shown here explicitly, each element of the Mueller matrix can be a function of wavelength, observation angle, and spatial coordinates. Like the Stokes vector, the Mueller matrix is normalized. This normalization is relative to element $m_{11}$. Thus, $m_{11}=1$, and the remaining elements have values on the interval $[-1,1]$.

Polarization properties accounted for in the Mueller matrix include diattenuation, retardance, and depolarization. Diattenuation describes the dependence of the intensity transmittance on the incident polarization state. Retardance denotes a polarization-dependent phase change, and depolarization represents the coupling of polarized light into unpolarized light [19].

Row one of the Mueller matrix $\left[\begin{array}{llll}m_{11} & m_{12} & m_{13} & m_{14}\end{array}\right]$ represents diattenuation, as this row indicates how the output intensity varies with the incident polarization state. Column one of the Mueller matrix $\left[\begin{array}{llll}m_{11} & m_{21} & m_{31} & m_{41}\end{array}\right]^{T}$ represents the Stokes vector of the output intensity for unpolarized incident light. Retardance typically 
can be associated with six specific elements $\left(m_{23}, m_{24}, m_{32}, m_{34}, m_{42}\right.$, and $\left.m_{43}\right)$, as these elements describe how a given polarization state transforms to other polarization states.

For example, the $m_{23}$ element represents how incident light polarized at $\pm 45^{\circ}$ is converted to linear horizontal or vertical polarization. The $m_{32}$ element does the opposite, as it transforms incident horizontal or vertical polarization to $\pm 45^{\circ}$ polarization. These two elements are sensitive to linear retardance oriented at $0^{\circ}$ and $90^{\circ}$. The $m_{24}$ element shows the conversion of circularly polarized incident light into horizontal or vertical linear polarization, while $m_{42}$ does the same conversion, but in the opposite direction. This element pair is sensitive to linear retardance oriented at $\pm 45^{\circ}$. The $m_{34}$ and $m_{43}$ elements convert circular polarization to $\pm 45^{\circ}$ linear polarization and $\pm 45^{\circ}$ linear polarization to circular, respectively. These elements exhibit sensitivity to circular retardance effects [19]. The diagonal elements $\left(m_{22}, m_{33}\right.$, and $\left.m_{44}\right)$ offer insight into depolarization, however, this is only true when the off-axis elements are at or near zero. Pure depolarization corresponds to the case in which all elements (except $m_{11}$ ) are zero. Partial depolarization corresponds to the case in which the diagonal elements have values on the interval $(0,1)[19]$.

If a system consists of a series of $q=1,2, \ldots, Q$ polarization elements, their respective Mueller matrices can be multiplied together to create a composite Mueller 




Figure 2.5: Schematic representation of a sequence of polarization elements with input and output Stokes vectors.

matrix $\mathbf{M}_{c}$ for the system (see Fig. 2.5) [19],

$$
\mathbf{S}^{\prime}=\mathbf{M}_{Q} \mathbf{M}_{Q-1} \ldots \mathbf{M}_{q} \ldots \mathbf{M}_{2} \mathbf{M}_{1} \mathbf{S}=\mathbf{M}_{c} \mathbf{S}
$$

In summary, the Stokes/Mueller formalism fully describes the polarization state of light and the polarization-altering properties of an optical component or medium as a function of wavelength, observation angle, and spatial coordinates. Because of the versatility of this calculus, it can be used for describing the polarization effects in a measurement system or media polarization parameters. Thus, it is appropriate for calibrating polarimeters and describing polarized spectral scatter in terms of additive intensities, which is the topic of the next section.

\subsection{Polarized spectral scatter}

The interaction of light with a medium can reveal important information regarding a medium's properties. A medium can either absorb, scatter, or transmit light, and these mechanisms can alter spectrally-dependent properties of light such as intensity, 
polarization, and propagation direction. The ability to interpret these alterations provides a method for nondestructive evaluation of medium properties. This section describes two specific case of the interaction between polarized light and a medium, the information these interactions can provide, and the method in which the information is obtained.

Polarized spectral transmission through a material is an example of the light/medium interaction. This type of measurement can reveal medium properties such as diattenuation, birefringence (linear retardance), optical activity (circular retardance), refractive index uniformity, or defects (voids, cracks, or bubbles) [19, 42]. Methods for obtaining these properties include polarizing light microscope measurements, Stokes vector measurements, or Mueller matrix measurements.

Many researchers have used these three measurement modalities for obtaining the aforementioned properties, but with limitations. For example, polarizing light microscopes typically do not provide quantitative data, and often require a trained specialist for interpreting the interference colors due to birefringence [43]. Stokes vector measurement systems typically do not measure the full Mueller matrix, thus they do not capture all polarization effects [19]. Many Mueller matrix systems exist (see Section 2.5.1, Measurement strategies), however, not all of them possess spectral capabilities. The spectral aspect of polarization is sensitive to small-scale structure, as a periodicity in the polarization response describes the structure [4]. 
Polarized spectral scatter from a material surface serves as another particular case of the light/medium interaction. Measuring various components of polarized spectral scatter can elucidate many properties about a surface, such as thin film thickness, complex refractive index, surface roughness, surface features (blemishes, scratches), surface or subsurface defects, particulate contamination, and grain boundaries $[41,44]$. Methods for obtaining the various components of polarized spectral scatter and the aforementioned medium properties can include ellipsometry [45-47], linearly polarized angular scatter measurements [48-54], or bidirectional reflectance distribution function (BRDF) measurements [55-58].

Although much research has been done to measure different features of polarized spectral scatter, minimal work has been done employing polarized spectral scatter for estimating the complete Mueller matrix for material characterization. Some of the polarized spectral scatter research using the Mueller matrix framework has been applied to measure the dielectric tensor of anisotropic materials $[16,59,60]$. Others have used Mueller matrix measurements to estimate critical dimensions of diffracting structures $[17,18,61]$. Also, the Mueller matrix framework has been used to characterize biological tissue in terms of surface roughness [11], depolarization [13], and retardance/diattenuation [62].

As this section shows, there are various cases of the light/medium interaction. With these various cases comes an equally diverse set of measurement techniques and methods of parameterizing the medium. However, generally speaking, probing the 
interaction between polarized light and a medium requires some type of polarimetric measurement configuration. The following section describes polarimetry and the fundamental concepts motivating this field.

\subsection{Polarimetry}

Polarimetry is the science of measuring the vector nature of light. A polarimeter is the device used to make these measurements. Fundamentally, all polarimeters perform the same task: measure the Stokes vector. Polarimeters can achieve this by acting as stand-alone devices, termed passive polarimetry, or in combination with a Stokes generator (a device used to generate specific incident polarization states), called active polarimetry [19]. Estimating the Mueller matrix requires active polarimetry, as specific Stokes vector measurements in combination with specific incident polarization states allow for the estimation of the entire Mueller matrix. Although there are polarimeters in many different configurations with an abundance of measurement strategies, optimization techniques, and components, the rest of the current section will focus on a general overview of polarimeters. Specific polarimeter characteristics will be discussed in the following two sections, 2.5.1 and 2.5.2.

In general, polarimeters consist of analyzing optics and a polarizationinsensitive detector (i.e., a photodiode or camera). Because of this insensitivity, it is only possible to directly measure the $I$ element of the Stokes vector. The remaining 
three elements $(Q, U$, and $V)$ must be inferred from additional measurements. As a result, polarimeters must possess the ability to make tunable polarization measurements, where the analyzing optics are altered for measuring the $I$ component of different polarization states. Manipulation of the measurements then leads to inference of the Stokes vector.

Conceptually, all polarimeters operate in a similar manner. Accurate Stokes vector estimation requires a series of intensity measurements at different polarimeter settings, where a composite Mueller matrix (see equation (2.7)) represents each polarimeter measurement. When using an intensity detector, only the $I$ element of the output Stokes vector can be measured. The $I$ element equals the inner product of the first row of the composite Mueller matrix and the input Stokes vector. Therefore, the detector output is equal to [63]

$$
I_{\text {out }}^{(n)}=m_{11}^{(n)} I_{\text {in }}+m_{12}^{(n)} Q_{i n}+m_{13}^{(n)} U_{i n}+m_{14}^{(n)} V_{i n}
$$

where the superscript $n$ denotes the polarimeter configuration for the $n^{\text {th }}$ measurement. A series of measurements then can combine to create a system of linear equations,

$$
\mathbf{V}=\mathbf{A S}
$$

where $\mathbf{V}$ is an $n \times 1$ vector of observed intensities, $\mathbf{A}$ is the $n \times 4$ analysis matrix with each row containing the first row of the $n^{\text {th }}$ composite Mueller matrix, and $\mathbf{S}$ is 
a $4 \times 1$ unknown Stokes vector. The analysis matrix $\mathbf{A}$ describes the $n$ configurations of the polarimeter. In principle, Stokes vector estimation can be achieved with four measurements (i.e., with four unique polarimeter configurations), but often more are made to increase precision.

Estimating the unknown Stokes vector typically involves inverting the analysis matrix A. This inversion is straightforward when $n=4$ measurements are made, as the matrix $\mathbf{A}$ is square. The inverted matrix $\mathbf{A}^{-1}$ is commonly known as the data reduction matrix (DRM) W such that $\mathbf{W}=\mathbf{A}^{-1}$. This procedure for estimating the Stokes vector is frequently called a data reduction technique, which reduces polarimeter characterization into a single matrix, the data reduction matrix [19]. The DRM can then be used to estimate the unknown Stokes vector,

$$
\hat{\mathbf{S}}=\mathbf{W V}
$$

For the case when $\mathbf{A}$ is not square, (i.e., $n>4$ ), then $\mathbf{S}$ is solved for in a least-squares sense,

$$
\hat{\mathbf{S}}=\left(\mathbf{A}^{T} \mathbf{A}\right)^{-1} \mathbf{A}^{T} \mathbf{V}
$$

where $\left(\mathbf{A}^{T} \mathbf{A}\right)^{-1} \mathbf{A}^{T}$ is the pseudoinverse of $\mathbf{A}$.

Regardless of the number of measurements made, determining the analysis matrix A requires knowledge about the polarimeter components, in terms of either a 
parameterization of the components, or the assumption of ideal component behavior. A technique for estimating the DRM by making $n>4$ measurements with no a priori knowledge about polarimeter component behavior is described in Section 3.4.1.

In conclusion, this section summarized the main points relevant to polarimetry, and described a method for obtaining the data reduction matrix. Arriving at the data reduction matrix can be achieved with various measurement strategies, as many have been devised in an attempt to accurately estimate the DRM. These strategies are the subject of the next section.

\subsubsection{Measurement strategies}

Although accurate Stokes vector estimation emerges as the principal goal common to all polarimeters, a wealth of measurement strategies exist in pursuit of realizing this goal. Measurement strategies can be categorized by many different criteria, such as the time scale for Stokes vector acquisition (series or parallel measurements), the means of modulating the phase relationships of polarization states, the components employed, or the spectral dependence of the measurements. These criteria are important as they emphasize the uniqueness of the system described in this dissertation. For this section, measurement strategies are discussed in terms of their components, and include systems based on rotating polarizers [64,65], rotating retarders [66-71], photoelastic modulators (PEMs) [72-77], birefringent uniaxial crystals [78-82], polarization gratings (PGs) [83-85], and variable retardance 




Figure 2.6: Schematic of the rotating polarizer measurement configuration.

devices $[2,10,11,13-15,17,18,51,60-63,86-98]$.

Measurement configurations constructed from rotating polarizers consist of a rotating polarizer in either the polarimeter or in both the Stokes generator and polarimeter (see Fig. 2.6) [40]. These configurations implement measurements in series, as polarization information is acquired sequentially. Most polarizers used in such applications are dichroic sheet polarizers, which absorb polarized light more strongly in one direction than in the orthogonal direction [38]. Thus, these devices ignore the relative phase between the orthogonal field components.

Rotating polarizer measurements can be implemented by either placing the polarizers at predetermined orientations [19] or by continuously rotating the polarizers [45]. The rotation encodes the Stokes vector elements into the frequency components of the detected signal. The primary advantages of these systems include a wide spectral range and the ability to easily implement an imaging configuration. However, rotating polarizer systems experience systematic error due to the polarizer mechanical movements and vibrations, and are limited to measuring only the Mueller 


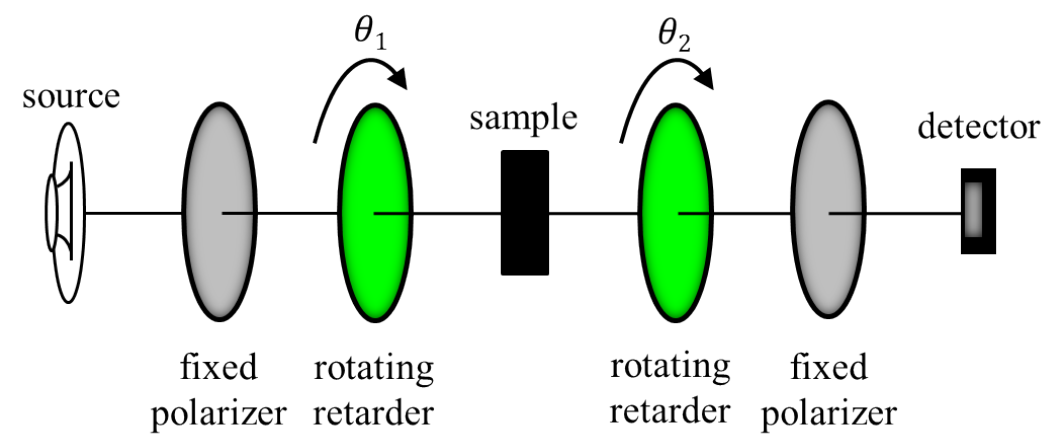

Figure 2.7: Schematic of the rotating retarder measurement configuration.

matrix elements associated with linear polarizations (the upper left $3 \times 3$ submatrix). Furthermore, these configurations are not suitable for rapidly varying samples.

Rotating retarder systems utilize a single rotating retarder in the polarimeter or a pair of rotating retarders (see Fig. 2.7), one each in the Stokes generator and the polarimeter (for Mueller matrix measurements) [40]. Retarders are birefringent elements that introduce a relative phase delay between orthogonal polarization components. Like the rotating polarizer systems, the rotating retarder systems acquire polarization information sequentially. However, the retarders in these systems introduce a phase shift between orthogonal polarization components. The retarders can be rotated to predetermined positions or rotate at a specific angular increment relative to each other, encoding the Mueller matrix elements into the Fourier components of the detected signal. Although the calibration and construction is relatively straightforward, the rotating retarders introduce vibration, limit acquisition time, and are not suitable for rapidly varying samples [68]. Additionally, they introduce beam wander, as some retarders have wedges between the crystal plates within the device that cause 
the image of a point source to trace out a circular path on the camera array $[66,67]$. This effect produces image misregistration and results in Stokes vector error.

Polarimetric systems based on photoelastic modulators (PEMs) also rely on various harmonics in the measured signal. PEMs operate by using transducers to apply oscillatory stress to a birefringent element inside the device, where this stress is applied at a fixed frequency in the $\mathrm{kHz}$ range. Systems utilizing PEMs introduce a phase shift between orthogonal polarization components while also making sequential polarization measurements. Systems based on these devices exhibit high sensitivity and signal-to-noise (SNR) characteristics due to high modulation frequencies and synchronous detection. Although synchronous detection requires a lock-in amplifier and a point detector, it is possible to use PEMs in imaging configurations. However, incorporating PEMs into imaging systems is not straight-forward, as the high frequency, free-running nature of the devices can not be tuned to accommodate relatively slower CCD cameras [75]. Such imaging systems can overcome these issues by employing time-gating techniques for synchronizing CCD measurements with the PEMs [76,77]. Additionally, systems using only two polarimeter PEMs can measure the Stokes vector, while systems employing four PEMs can measure the full Mueller matrix [74]. Shortcomings of PEMs include misalignment effects and frequency drift [77].

Systems employing birefringent uniaxial crystals act as Fourier transform imaging spectrometers (FTIS). These systems, also called birefringent or polarization interferometers, differ from conventional FTIS in that they achieve interference effects 




Figure 2.8: Illustration of a Wollaston prism producing orthogonally polarized outputs exhibiting shear.

from a common path configuration (i.e., the reference and sample beams follow the same optical path) instead of the more traditional Michelson interferometer configuration (i.e., the reference and sample beams follow different paths).

Birefringent interferometers typically employ various combinations of optically anisotropic elements, such as wedge prisms [78], Savart plates [79-81] or Wollaston prisms [82]. In general, these systems use a birefringent element to produce shear between the orthogonal polarization states of the incident beam. Figure 2.8 shows a schematic of a Wollaston prism, which consists of a pair of wedge prisms cemented together. A Wollaston prism splits incident light into two orthogonally polarized outputs with a specific angular divergence, where the prism wedge angle controls the divergence. Savart plates are different as they are constructed from a pair of equal-thickness birefringent plates cemented together. This construction results in orthogonally polarized outputs that exhibit shear, or a lateral displacement [99]. In terms of the inteferometers, the sheared beam is analyzed with additional birefringent elements (that are either static or dynamic), and then the beams are recombined to interfere at the detector plane. 
Static birefringent interferometers have used wedge prisms [78] or Savart plates [79]. These components generate four collinearly propagating, sheared beams that are recombined at the image plane to form an intensity inteferogram with spatial carrier fringes. Encoded in the fringes are spatially-dependent Stokes vectors. Fourier transforming the interferogram produces the power spectrum, which contains a series of peaks that represent the Stokes vector elements. Filtering the peaks in the spatial frequency domain, followed by Fourier inversion, produces the Stokes vector elements. These systems typically have been used only with quasi-monochromatic light, as fringe visibility decreases with decreasing temporal coherence length of the incident light [100].

Dynamic systems utilize either a pair of moving wedge prisms $[80,81]$ or moving Wollaston prisms [82] and can be used with broadband illumination. The prisms produce shear between orthogonal polarization states, which are then combined by a polarizer and lens to form an interferogram at each pixel in the image plane. Translating one of the prisms through the beam introduces a variable optical path difference (OPD) between the two sheared beams, thus producing a temporal interferogram at each pixel. This interferogram is a function of the OPD. Fourier transforming the interferogram and then filtering gives the spectral and polarization content at each pixel.

Both the static and dynamic systems employing birefringent uniaxial crystals have advantages and drawbacks. In general, these common-path systems are robust, 
as they exhibit stability (relatively insensitive to vibrations) and simplicity (typically fewer optical components) [78-82]. However, static systems exhibit wavelengthdependent carrier frequencies. Therefore, a wide optical bandwidth produces multiple carrier frequencies, thus reducing fringe visibility. In order to obtain high visibility fringes, narrow bandpass filters must be used [100]. As a result, static systems typically suffer from limited spectral information (or the need for multiple wavelength filters) and low signal-to-noise ratios. On the other hand, efforts have been made for reducing the bandwidth limitation by introducing dynamic prisms, but this creates vibration sensitivities [100]. Finally, these systems also suffer from information loss during the Fourier filtering phase. For example, in the spatial frequency domain of static systems, the spatial frequency resolution can be inadequate. This causes overlap of the channels that represent the Stokes vector elements, resulting in cross-talk and information loss [79].

Polarization grating (PG)-based systems rely on diffractive polarization effects to provide shear. A PG functions similarly to a Wollaston prism as it diffracts light into forward-propagating, orthogonal polarizations [83]. Similar to systems using uniaxial crystals, PG-based systems encode the Stokes parameters into amplitude modulated carrier frequencies. PG-based systems employ a series of polarization gratings, such that the first PG diffracts the incident beam into orthogonal polarization states in the \pm 1 diffraction orders, then subsequent PGs introduce spectrally-dependent shear. Measuring linear polarization states requires two PGs, while measuring the 
full Stokes vector requires four PGs and a quarter wave plate [84,85]. An analyzer and lens recombine the sheared beams at the image plane, producing spatially modulated interference fringes, which contain the polarimetric information. PG systems can be used in broadband applications and can acquire spectral polarization information in a single snapshot. However, they also suffer from reconstruction artifacts due to aliasing. In other words, the channels containing the individual Stokes vector components overlap, causing an unwanted interaction between adjacent channels. Furthermore, the zero-order transmission of the PGs can cause error in the reconstructed Stokes vector, and like the birefringent uniaxial systems, suffer from reduced information content due to spatial frequency filtering.

Variable retarder systems rely on birefringent liquid crystal devices to alter the incident polarization state with no mechanically moving hardware. These devices impart a phase delay between orthogonal polarization components based on the tilt angle of the LCs inside the device. Two specific types of variable retarders used in polarimetric systems are ferroelectric liquid crystal cells (FLCs) $[18,61,87,89]$ and liquid crystal variable retarders (LCVRs) [2,10,11,13-15,17,51,60,62,63,86,88,90-98]. Systems using these two types of devices can be optimized at multiple wavelengths and can acquire a complete Mueller matrix on the order of seconds, but suffer from temperature and alignment sensitivities.

The LCs in FLC devices possess permanent dipole moments, where the moments can be reversed to change the effects produced by the device. This results in a 
birefringent device with switchable fast axis orientations that are $45^{\circ}$ apart. Typically, FLCs are manufactured to act as either switchable half-wave plates or switchable quarter-wave plates [101] and can modulate settings more quickly than LCVRs [89]. Systems using four FLC devices (a pair in both the Stokes generator and the polarimeter) can measure the complete Mueller matrix $[87,89]$.

On the other hand, LCVRs can apply a wide range of retardances, ranging from 0 to $2 \pi$ radians for a given wavelength. A polarimeter consisting of two LCVRs can estimate a Stokes vector $[2,11,14,15,51,63,86,90,92-95,97]$, while measuring the complete Mueller matrix requires two additional LCVRs in the Stokes generator [13, 17,60,62,88,91]. Moreover, LCVR variability permits better polarimeter optimization than FLC-based systems, as FLC devices have a limited number of degrees of freedom. In other words, once the FLC fast axis orientation is selected, the second orientation is dictated by the device. Thus, FLC polarimeters are limited to two degrees of freedom, resulting in only four analyzer configurations. Conversely, LCVR polarimeters have four degrees of freedom, providing more than four analyzer configurations. Because of this, LCVR-based systems display better noise propagation effects than FLC systems [89].

As this section shows, many different measurement strategies have been devised for estimating the Stokes vector. However, LCVR-based polarimetry emerges as a measurement strategy with advantages over the others. For example, LCVRbased systems exhibit no mechanically moving hardware that can cause vibrations 
or beam wander, as in rotating polarizer and rotating retarder systems. Systems using LCVRs also have no loss of information due to Fourier filtering as experienced by birefringent uniaxial systems and PG-based systems. Additionally, LCVRs are computer-controlled, which is in contrast to PEMs, as these devices operate at a fixed frequency and are not tunable (i.e., they operate at a fixed frequency). Finally, relative to FLCs, systems using LCVRs can be optimized more effectively. Regardless of the measurement strategy, the next step is optimizing the polarimeter, which is the topic of the ensuing section. Because this research focuses on LCVR-based polarimetry, optimization is discussed in terms of this type of system.

\subsubsection{Optimization}

Optimization consists of determining the polarimeter configuration and set of measurements that best suppress error amplification and propagation. As mentioned previously, Stokes vector estimation involves solving a system of linear equations. Therefore, polarimeter optimization is analogous to improving the condition of the system of equations (i.e., the analysis matrix A). System conditioning refers to the sensitivity of a linear system of equations to errors, and how those errors propagate [90]. Subsequently, optimization strives to determine the system of equations that mitigate the effects of random error (measurement noise) and systematic error (individual component uncertainty). Another factor influencing optimization includes the calibration method employed. Typically data reduction calibration techniques 
focus on determining a calibration matrix (i.e., the DRM) that describes the polarimeter. In contrast, another technique, the eigenvalue calibration method (ECM), emphasizes measurements on specific calibration samples rather than polarimeter design. In other words, the ECM utilizes a series of measurements on several known samples to generate a calibration matrix for the polarimeter [102]. Due to the broad range of measurement strategies, optimization will be discussed here only in terms of non-Fourier based systems, with an emphasis on variable retardance polarimeters. An extensive collection of optimization techniques for other measurement strategies can be found elsewhere $[2,45,63-65,68-74,103,104]$.

As noted previously, system optimization can be quantified in terms of the condition number [63]. The condition number is a matrix property that describes its nearness to singularity. A minimum condition number indicates that the linear independence of rows and columns is maximized [105]. The condition number $\kappa$ is defined as

$$
\kappa(\mathbf{B})=\|\mathbf{B}\|_{\rho}\left\|\mathbf{B}^{-1}\right\|_{\rho},
$$

where $\left\|\mathbf{B}^{-1}\right\|_{\rho}$ is the $p$-norm of matrix $\mathbf{B}$. The user is free to choose the matrix norm; here the $L_{2}$ norm is used. By definition, the $L_{2}$ norm allows one to calculate the condition number from the ratio of the largest to smallest singular values of the matrix $\mathbf{B}$ [106]. Thus, the condition number can be expressed as

$$
\kappa(\mathbf{B})=\frac{\sigma_{\max }}{\sigma_{\min }},
$$


where $\sigma_{\max }$ and $\sigma_{\min }$ are the maximum and minimum non-zero singular values of B. Although the condition number functions as an important optimization metric, it provides only generalized guidance for the design process [98]. Another metric, measuring the Mueller matrix of air, provides a more detailed look at the design process. This construct, which is ideally the identity matrix, is sensitive to systematic error in the system. In other words, specific types of systematic error can cause a particular type of error signature in the Mueller matrix of air (see Section 3.4.2 for more details). Finally, measuring the Mueller matrix of air indirectly assesses system conditioning, as lower errors associated with this matrix suggest not only a wellcalibrated polarimeter, but also a well-conditioned system.

System condition depends on the number and type of polarimeter measurements, along with the configurations used in making the measurements. Many groups have focused on mathematical simulations for determining optimum configurations and measurements. However, there also have been a number of researchers who have used a geometrical interpretation involving the Poincaré sphere for arriving at optimum polarimeter configurations and measurements $[70,95,103,104]$.

In the Poincaré sphere interpretation, one can envision each measurement configuration as producing a Stokes vector plotted on the Poincaré sphere. Interpreting these Stokes vectors as the vertices of a generally irregular polyhedron leads to the inscription of the polyhedron inside the Poincaré sphere. The volume of the polyhedron is inversely proportional to the condition number. Selecting maximally 
spaced vertices leads to a maximum polyhedron volume, thus forming a regular polyhedron $[2,90,98]$. Therefore, polarimeter design can be limited to configurations and measurements that only inscribe regular polyhedrons inside the Poincaré sphere, thus minimizing error propagation. One is able to make $n \geq 4$ optimum measurements as long as they maximize the regular polyhedron volume. Implementing more than four measurements can mitigate random error, which introduces redundancy and increases the SNR by a factor of $\sqrt{n}[2]$. Variable retardance polarimeters can access the entire Poincaré sphere surface, resulting in a continuum of optimum configurations [98]. Tyo has shown that for variable retardance polarimeters consisting of two LCVRs, the optimum condition number can be achieved for retarder fast axis orientations of $0^{\circ}$ and $45^{\circ}$, which also gives access to the entire Poincaré sphere $[63,98]$.

While most polarimeter optimization schemes focus on configuration design, as shown in the previous paragraph, some researchers have investigated calibration techniques as well, as this choice poses another method of minimizing error propagation. Calibration techniques emerging as the most prevalent include the eigenvalue calibration method (ECM) [102], data reduction [93], and fine-adjustment of drive voltage settings for variable retardance polarimeters [97]. The ECM uses three characteristic Mueller matrices to represent the Stokes generator, sample, and the polarimeter. By acquiring up to four measurement sets using four different reference samples, one can recover Mueller matrices for the Stokes generator and polarimeter. Then, the system Mueller matrices can be combined with subsequent sample measurements to 
determine the sample Mueller matrix. This technique requires no a priori information about the Stokes generator or polarimeter. Limitations of this technique include aligning and then switching the reference samples, and the requirement that the Mueller matrices of the four reference samples (two smooth, isotropic surfaces and two linear polarizers) be known perfectly.

Data reduction techniques seek to use a $4 \times n$ data reduction matrix $\mathbf{W}$ to estimate an arbitrary Stokes vector, based on $n$ measurements. In the general case, the DRM is computed by taking the pseudoinverse of the $n \times 4$ analysis matrix $\mathbf{A}$. When $n>4$, the pseudoinverse is computed in a least-squares sense. This technique requires an a priori estimate of the analyzing components in order to determine the analysis matrix A. This estimate can be in the form of either component parameterization or assuming ideal component behavior. However, optical components are imperfect, and physical effects, such as misalignment and reflections, can cause uncertainties in the analysis matrix. Boulbry et al. [93] have established a data reduction technique that requires no a priori knowledge of polarimeter components. This technique makes no polarimeter assumptions other than linearity, thus shifting systematic error onto the Stokes generator. Additionally, this technique requires presenting a series of known Stokes vectors (the training set) to the polarimeter. Measurements on the training set establish a relationship between the Stokes generator and polarimeter, from which the DRM can be computed using the singular value decomposition (SVD) [105]. The SVD is a matrix factorization method often used for solving ill-conditioned inverse 
problems, and can minimize random error through truncation. A more in-depth discussion of this technique and the SVD is presented in Section 3.4.1 and Appendix C, respectively. The SVD is selected as a computational tool for aiding in the calibration method for the current research.

In conclusion, this section provided an overview of polarimeter optimization, with an emphasis on variable retardance polarimeters. These polarimeters, which consist of two LCVRs, can be configured such that the optimum condition number is achieved with $n \geq 4$ measurements and device fast axes of $0^{\circ}$ and $45^{\circ}$. Variable retardance devices, such as LCVRs, are founded on liquid crystal technology. This technology, along with LCVRs and an additional liquid crystal device, will be discussed in the following section.

\subsection{Liquid crystal technology}

Liquid crystal technology has seen rapid development due to the growth of display applications in the electronics industry, such as in televisions, laptops, and mobile phones. This development also has expanded into the photonics industry, as the wide variety of liquid crystals, along with their unique electro-optic properties, make them suitable for a broad range of photonics devices [21]. Two such devices of interest are based on nematic liquid crystals (see Fig. 2.9), the liquid crystal variable retarder 


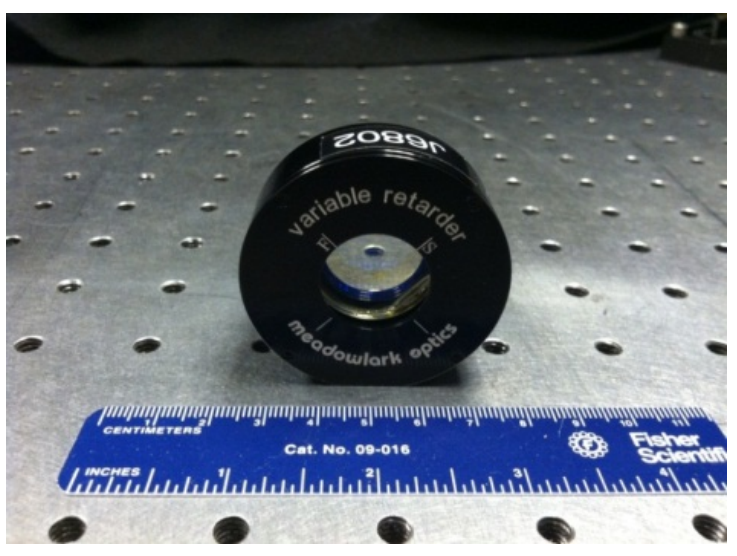

(a) LCVR

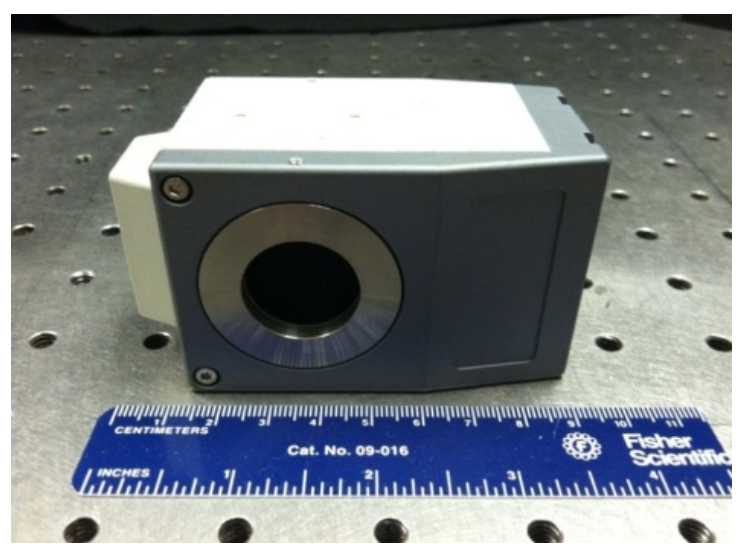

(b) LCTF

Figure 2.9: Nematic liquid crystal polarization devices.

(LCVR), manufactured by Meadowlark Optics, and the VariSpec liquid crystal tunable filter (LCTF), manufactured by Cambridge Research \& Instrumentation, Inc. (CRI). Liquid crystals, LCVRs, and the LCTF will be discussed in the following sections.

\subsubsection{Liquid crystals}

Liquid crystals are a state of matter that exhibit both the properties of crystalline solids and isotropic liquids. The particles in the liquid crystal phase exhibit fluidity and an inability to support shear, but also some degree of periodic order as in a crystalline material. The degree of order determines the anisotropy of the LC optical and electrical properties [107]. Although a variety of LC phases exist, the particles of the different phases tend to have certain features in common, such as elongated or disc-like shapes on the nanometer size scale, and are easily polarizable [107]. 
The many types of LCs are classified primarily in terms of their ordering. A unique type is the nematic liquid crystal, which is uniaxial and rod-like. They exhibit a high degree of long-range orientational order, but due to their random positioning, lack positional order $[107,108]$. Nematic LCs, and in general all LCs, are sensitive to external stimuli, such as electric fields and alignment layers on the

enclosure surfaces $[107,108]$. The ability to control external stimuli leads to increased LC ordering. The increased order and the ability to control it in nematics creates strong, tunable birefringence effects, which are useful in optics applications.

\subsubsection{Liquid crystal variable retarders}

Liquid crystal variable retarders employ a user-controlled drive voltage to adjust the effective birefringence of the device, thus allowing change of an incident polarization state. These devices, filled with a birefringent nematic liquid crystal material, impart retardance effects on a millisecond time scale with no mechanically moving hardware. The retardance is computer-controlled and can vary up to a full wave for a given wavelength. These effects are due to the particular construction, discussed in the following paragraphs.

A schematic of the Meadowlark LCVR is shown in Fig. 2.10 [109]. These devices contain a proprietary nematic liquid crystal material, which is sealed in a cavity constructed from two fused silica windows. The cavity is on the order of $\approx 10$ $\mu \mathrm{m}$ thick, while the windows have a clear aperture of $\approx 18 \mathrm{~mm}$ in diameter. Each 


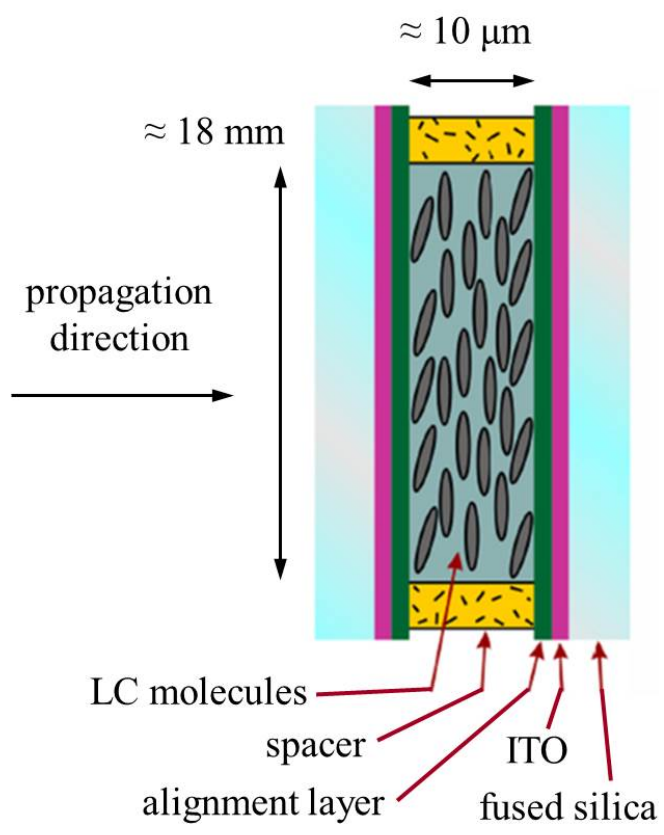

Figure 2.10: Meadowlark LCVR construction. Figure taken from [109]. Not to scale. window is coated with an alignment layer and a current-conducting layer, indium tin oxide (ITO). The alignment layer is a thin dielectric that is applied and then gently rubbed, where the rubbing produces grooves that cause the LCs to have a particular alignment. Electrodes are attached to the windows to create a voltage difference across the cavity, thus establishing the electric field across the LCs [109].

LCVRs function by delivering a user-controlled, $2 \mathrm{kHz}$ square-wave AC waveform (i.e., an electric field) across the LCs. The waveform has a zero-average voltage and a peak-to-peak voltage that ranges from zero to \pm 10 volts [109]. Employing an $\mathrm{AC}$ waveform prevents damaging ionic buildup form occurring in the LC layer. AC waveforms are used because a DC waveform can cause the injection of supplementary charge carriers, which leads to alignment and positioning instabilities. Furthermore, 
DC waveforms can lead to chemical degradation of the nematic mixture [110]. The peak-to-peak voltage controls birefringence effects.

Varying the peak-to-peak voltage (also known as the drive voltage) allows the user to control LC orientation and hence the effective birefringence. The electric field causes the LCs in the liquid crystal layer to undergo a global, uniform orientation change, thus changing the applied retardance of the device. Specific retardance values correspond to a drive voltage as defined by the unique tuning curve of the LCVR. At lower drive voltages, the LCs are approximately perpendicular to the optical axis, and thus display a higher birefringence. At higher drive voltages, the LCs rotate and become parallel to the optical axis, decreasing the birefringence. LCs at the windows, however, are not free to rotate because they are pinned by the windows. This surface pinning causes a residual retardance, which keeps the LCVR from reaching zero retardance. To achieve zero retardance, LCVRs have a fixed polymer retarder, called the compensator, attached to the liquid crystal cell. The compensator, unlike the LCs, has a constant retardance value that does not change with drive voltage.

LCs exhibit a particular orientation inside the LCVR cavity, based on the alignment layer and drive voltage. The director, a dimensionless unit vector parallel to the long axis of the LCs, describes this preferred orientation [107,108]. The long axis of the LCs also has a higher refractive index relative to the short axis. Thus, the LC long axis is the slow axis of the device, and coincides with the director. The angle of the director projection onto the transverse $(x, y)$ plane defines the slow axis 




Figure 2.11: Schematic of the liquid crystal director and the fast and slow axes. The director is parallel to the long axis of the LCs (which is also the slow axis), and describes their preferred orientation.

(see Fig. 2.11). Additionally, the director defines the LC tilt angle, as the tilt angle is between the director and the optical axis. Figure 2.12 shows the tilt angle for a single liquid crystal. Values of the tilt angle range from $\approx 0^{\circ}$ at a drive voltage of 0 volts to values of $\approx 90^{\circ}$ at 10 volts. The tilt angle as a function of drive voltage $\beta(V)$ is related to the liquid crystal layer retardance through the expression [111]

$$
\beta(V)=\cos ^{-1}\left(\frac{\delta_{L C}(V)}{\delta_{\max }}\right)^{1 / 2}
$$

where $\delta_{L C}(V)$ is the liquid crystal layer retardance and $\delta_{\max }$ is the maximum LCVR retardance given by $\delta_{\max } \approx\left(\delta_{10 \text { volts }}-\delta_{0 \text { volts }}\right)$.

In summary, LCVRs exhibit unique electro-optic properties based on the characteristics of the LC mixture inside the device. For example, the nematic LCs used by the LCVRs exhibit optical anisotropy, a high degree of orientational order, and a high 



Figure 2.12: Schematic of the liquid crystal director and the liquid crystal tilt angle. The fast axis is parallel to the $x$-axis, which is perpendicular to the page.

sensitivity to external stimuli. These characteristics, along with the tunability of the LCVRs, make these devices attractive options for spectral polarimetric applications. Another LC-based device, the liquid crystal tunable filter, is discussed next.

\subsubsection{Liquid crystal tunable filters}

Liquid crystal tunable filters transmit a specific wavelength and exclude others by electronically controlling LC elements based on a user input. LCTFs essentially act as an interference filter that combines the technology of LCVRs and Lyot filters [112]. Like LCVRs, the LCTF is computer-controlled with millisecond switching times and no mechanically moving hardware. Using the LCTF consists of simply selecting a transmission wavelength. The remainder of this section describes the construction and functionality of the LCTF.

The precursor to the LCTF, the Lyot filter, utilizes a series of birefringent 


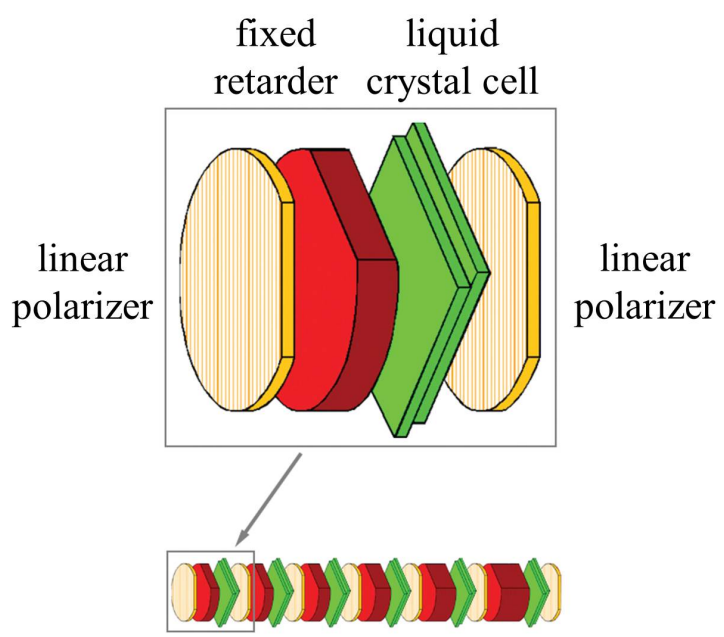

Figure 2.13: Schematic of a single Lyot filter stage built using a liquid crystal element. Figure taken from [112].

plates of different thicknesses and orientations. This configuration imparts phase shifts between orthogonal polarization components, resulting in a unique transmission profile after each plate. The Lyot filter ultimately transmits a specific bandwidth of wavelengths [21]. See Appendix D for a more detailed explanation of the Lyot filter.

The VariSpec LCTF, based on the Lyot filter, consists of a series of birefringent stages. Each stage includes a polarizer, a fixed retarder, and a nematic liquid crystal cell (see Fig. 2.13) [112]. Varying the voltage across the LC cells shifts the transmission spectrum of each stage, which permits wavelength tunability. The voltage changes are automated, and are based on a user input. The LCTF has a clear aperture of $20 \mathrm{~mm}$ and a full width at half maximum bandwidth of $7 \mathrm{~nm}$ [113].

In closing, the main benefits of the LC devices are their millisecond switching times, no mechanically moving hardware, a wide range of tunable states, and 
electronic control. Drawbacks include LCVR alignment and temperature sensitivities, and low transmission at shorter wavelengths for the LCTF. Despite the drawbacks, these two devices are used in the polarimeter. LC device employment is discussed in the next chapter, which describes system construction and calibration. 


\section{System construction and assessment}

\subsection{Introduction}

Spectral imaging goniometric polarimeter measurements provide spatially-dependent polarization information as a function of wavelength and observation angle. Proper quantitative interpretation of the spectral, spatial, angular, and polarization content revealed by the measurements relies heavily on the accuracy and sensitivity of the system. Thus, a thorough understanding of the individual component functionality and the overall system functionality is required. Understanding arises from exercises such as individual component characterization, system calibration, sensitivity simulations, and test measurements. Additionally, a detailed understanding of the theoretical framework describing the system is necessary.

The rest of this chapter addresses the aforementioned topics, but first begins with an overview of the system configuration. This overview provides context for component functionality and location in the system. After the system configuration overview, the discussion proceeds with a description of the component characterization techniques. These techniques include those for characterizing the polarizers, 
LCTF, and LCVRs. After component characterization is the system assessment, which includes a description of polarimeter calibration, simulations for assessing LCVR alignment and temperature sensitivities and their impacts on system performance, and test measurements for determining accuracy and sensitivity.

\subsection{System configuration}

The spectral imaging polarimeter configuration consists of two modules, an illumination module (Fig. 3.1, top) and a detection module (Fig. 3.1, bottom). Figure 3.2 shows a photograph of the complete measurement configuration. The illumination module delivers a specific polarization state to the sample at a given wavelength. It achieves polarization control by employing a Stokes generator (constructed from two LCVRs), and wavelength selection by employing an LCTF. This module operates based on Köhler illumination [114], which generates homogeneous illumination at the sample plane and ensures that an image of the illumination source is not visible in the image plane (see Appendix F for more details). Additionally, Köhler illumination provides some degree of control over the illumination coherence [114].

The initial section of the illumination module consists of a broadband light source (Dolan-Jenner DC950 illuminator, 150 Watt, $6 \mathrm{~mm}$ diameter fiber) followed by a $25 \mathrm{~mm}$ focal length lens, and then a variable-diameter iris. The iris acts as a field stop and changes the size of the illumination region. Next, a VariSpec LCTF 




Figure 3.1: Schematic of the measurement configuration. The illumination module is on top and the detection module is on bottom.

(Cambridge Research \& Instrumentation, Inc.) with a $7 \mathrm{~nm}$ bandwidth permits wavelength selection in the spectral range of $400-720 \mathrm{~nm}$. A Newport broadband polarizing beam splitter (PBS) follows and ensures horizontally polarized light is incident on the Stokes generator. After the PBS is a $170 \mathrm{~mm}$ focal length lens to approximately collimate the light. Following the lens are the Stokes generator components, LCVR 1 and LCVR 2 (Meadowlark Optics, LRC-200). LCVR 1 has a fast axis of $45^{\circ}$ with respect to the $x$-axis of the laboratory reference frame, while LCVR 2 is mounted with its fast axis at $0^{\circ}$. Following the second LCVR is a long working distance, 20x infinity corrected objective lens (Mitutoyo), which focuses the light onto a sample. This objective has a working distance of $30.5 \mathrm{~mm}$. Typically, for Köhler illumination, a second variable-diameter iris is placed before the objective lens, where the iris acts as an aperture stop to specify the angular extent of the 


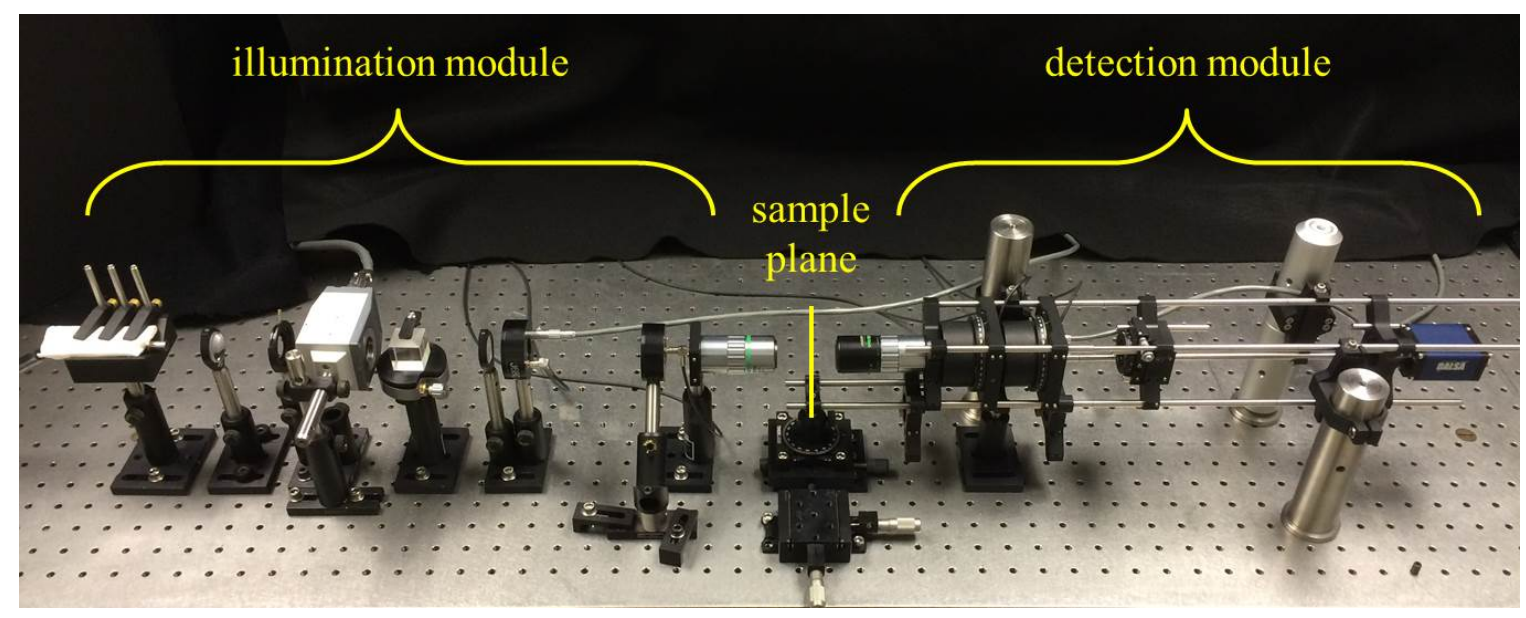

Figure 3.2: Photograph of the complete measurement configuration.

illumination cone at the sample plane. Here, the rear aperture $(\approx 5 \mathrm{~mm}$ in diameter $)$ of the objective lens acts as the aperture stop. It determines the apparent angular subtense of the source and thus the spatial coherence length [114].

The detection module begins after the sample plane and contains the polarimeter and a CCD camera. The polarimeter is constructed from a separate pair of LCVRs, while the camera images the polarized spectral scatter. The first element of the detection module is another long working distance, 20x infinity corrected objective lens (Mitutoyo), with a working distance of $20.5 \mathrm{~mm}$. Following the objective are LCVRs 3 and 4 (Meadowlark Optics, LRC-200), with fast axes at $0^{\circ}$ and $45^{\circ}$, respectively. Next is an analyzer oriented at $90^{\circ}$ with respect to the $x$-axis (i.e., vertically polarized). After the analyzer is a lens $(\mathrm{f}=200 \mathrm{~mm})$ and finally a CCD camera (Dalsa Genie M640-1/2). The detection module, enclosed in a cage system, rotates around the sample plane and provides the ability to perform angular scatter measurements. The cage system is connected to a rotation stage, where the stage is mounted directly 
under the sample. A MATLAB routine controls the electro-optical components and CCD camera. For example, based on user inputs, the routine executes commands for cycling through a series of LCTF wavelengths and LCVR retardance settings. While the routine cycles through wavelength and polarization settings, it also directs the CCD camera to acquire an image at each incremental step through wavelength and polarization. Ultimately, this routine acquires an intensity data cube that is a function of spatial coordinates, wavelength, incident polarization state, and polarimeter setting, all for a given observation angle.

In conclusion, this section described the components employed in the overall measurement system and their functionality. The next section addresses individual component characterization, along with the measurement configurations used for obtaining these characterizations.

\subsection{Component characterization}

Component characterization refers to quantifying the specific functionality of a given component. Characterization can result in the estimation of parameters, which describes the functionality. For example, polarizer characterization determines the spectrally-dependent principal intensity transmittances $k_{1}^{2}$ and $k_{2}^{2}$. LCTF and LCVR characterization consists of measuring the wavelength-dependent transmittance in order to determine the spectral response of the devices and their SNR. Another aspect 
of LCVR characterization includes obtaining the tuning curve and assessing tuning curve sensitivity to temperature and alignment. Once these characteristics are determined, system calibration can be performed.

\subsubsection{Transmittance measurements}

Transmittance measurements provide information describing the wavelengths with the highest SNR. This information aids in determining the most effective spectral window to use for the overall system. Transmittance measurements were made on the polarizer, LCTF, and LCVRs. Due to the complexity in determining the principal transmittances of the polarizers, this topic is described separately in Appendix G. The liquid crystal device measurements are discussed next.

A schematic of the system for measuring the LCTF and LCVR transmittances is shown in Fig. 3.3. The measurement uses the same illumination configuration as the overall system configuration (i.e., Köhler illumination), with the exception that the 20x objective lens is replaced with a longer focal length lens and a variable-diameter iris. Köhler illumination permits control of the beam diameter for the measurement, even though these measurements do not require this type of control.

The configuration consists of the broadband source, a $25 \mathrm{~mm}$ focal length lens, an iris, a $170 \mathrm{~mm}$ focal length lens, another iris, and a $200 \mathrm{~mm}$ focal length lens. The ensuing components are the component to be characterized and an Ocean Optics 


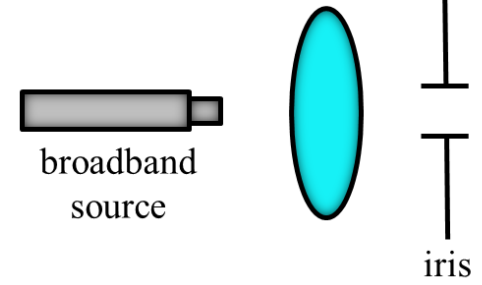

(field stop)

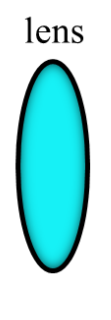

Figure 3.3: Schematic of the component transmittance measurement.

spectrometer, model HR2000+ES (14 bit). Computing the transmittance involves making three measurements. The first is a measurement on the component, and the other two measurements are a noise measurement with the light source blocked and a measurement with the component removed. The transmittance is calculated from the equation

$$
T(\lambda)_{\text {component }}=\frac{I(\lambda)_{\text {component }}-I(\lambda)_{\text {noise }}}{I(\lambda)_{\text {lamp }}-I(\lambda)_{\text {noise }}}
$$

where $I(\lambda)_{\text {component }}$ is the intensity transmitted by the component, $I(\lambda)_{\text {lamp }}$ is the signal the spectrometer detects in the absence of the component, and $I(\lambda)_{\text {noise }}$ is the spectral noise measurement made by blocking the light source.

For the LCTF measurements, the LCTF is cycled from $400 \mathrm{~nm}$ to $720 \mathrm{~nm}$ in increments of $20 \mathrm{~nm}$. At each LCTF wavelength setting, the spectrometer uses a $1 \mathrm{~ms}$ integration time and averages 1,000 spectra to produce one output spectrum. A MATLAB routine controls the LCTF and data acquisition. This routine cycles through the wavelengths on the LCTF, and at each wavelength, saves a text file of the spectrum acquired by the spectrometer. 
The LCVR transmittance measurements consist of spectrometer settings that use a $1 \mathrm{~ms}$ integration time and 5,000 averaged spectra to produce one output spectrum. A Matlab routine controls data acquisition, where the routine saves a text file of the spectrum acquired by the spectrometer. The drive voltage and temperature did not impact LCVR transmission, thus the LCVR settings were not changed.

\section{Results}

The principal transmittances $k_{1}^{2}$ and $k_{2}^{2}$ for the Newport sheet polarizers (model 10LPVIS-B) are shown in Fig. 3.4 (see Appendix G for a description of the measurement configuration and model). The polarizers exhibit poor performance at the shorter wavelengths. However, the polarizer characterization matches the manufacturer's specifications. Newport cites a minimum extinction ratio $\left(k_{1}^{2} / k_{2}^{2}\right)$ of 700 and an extinction ratio at $633 \mathrm{~nm}$ of 4,000. According to Fig. 3.4, the minimum extinction ratio is 424 and the extinction ratio at $633 \mathrm{~nm}$ is 4,071 . The discrepancy could be due to a lower lamp signal at the shorter wavelengths, thus producing a lower SNR.

The spectral transmittance $T(\lambda)_{\mathrm{LCTF}}$ for unpolarized illumination is shown in Fig. 3.5 and agrees well with the manufacturer's calibration. According to the figure, the LCTF has higher transmittances at longer wavelengths. At $400 \mathrm{~nm}$, transmittance is $0.4 \%$, and at $700 \mathrm{~nm}$, transmittance is $25 \%$. It should be noted that the data in Fig. 3.5 represent transmittance for unpolarized illumination. For polarized illumination parallel to the LCTF transmission axis, the transmittance doubles. 


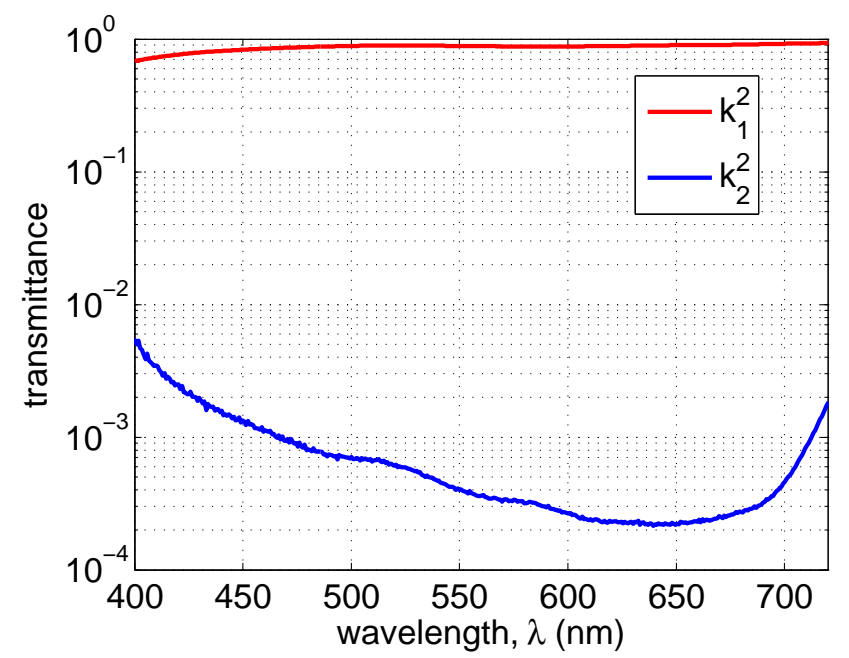

Figure 3.4: Principal transmittances for the Newport polarizers.



Figure 3.5: LCTF spectral transmittance. 


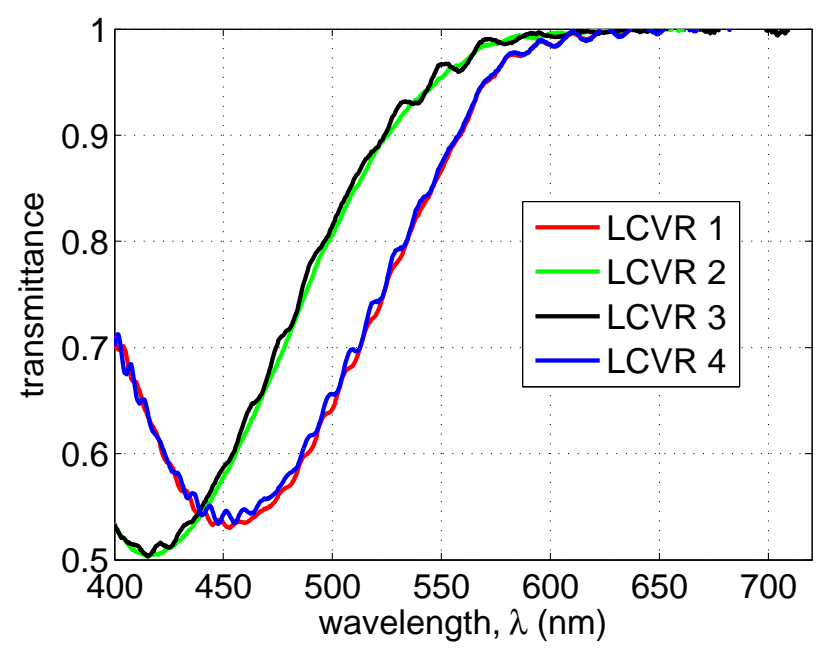

Figure 3.6: Spectral transmittance for the four LCVRs.

The spectral transmittance $T(\lambda)_{\mathrm{LCVR}}$ for each LCVR is shown in Fig. 3.6. This agrees well with the manufacturer's specifications, as LCVRs 1 and 4 have wavelength coatings for the $650-950 \mathrm{~nm}$ spectral region, while LCVRs 2 and 3 have coatings for the $450-700 \mathrm{~nm}$ spectral region. The ripple structure in the transmittances could be a result of etalon effects associated with the LCVRs. As with the other components, LCVRs exhibit poorer transmittance performance at shorter wavelengths.

\subsubsection{LCVR tuning curve calibration}

LCVR calibration results in a device-specific function that maps a drive voltage to a retardance. This tuning curve is acquired by placing an LCVR between crossed polarizers and measuring the transmitted intensity. The measured intensity corresponds to the phase delay between the orthogonal polarization components of the incident illumination. Converting the measured intensity to retardance produces the tuning 


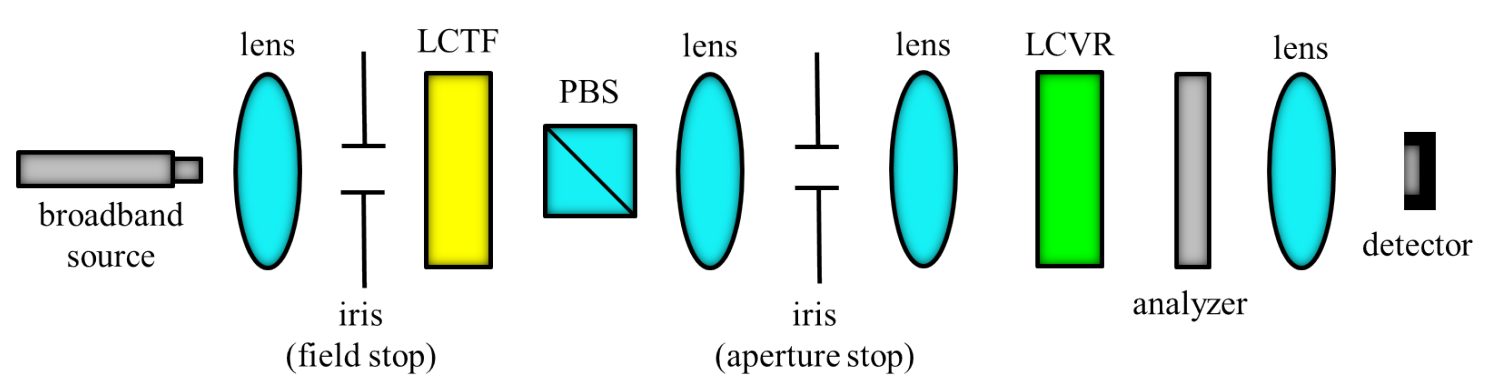

Figure 3.7: Schematic of the calibration configuration for each LCVR.

curve. Because the liquid crystals exhibit dispersion, tuning curves are obtained at multiple wavelengths.

Due to temperature and alignment sensitivities (discussed in the next section), each tuning curve corresponds to a unique set of conditions. Figure 3.7 shows a schematic for acquiring the tuning curves. The illumination portion of the configuration is a slightly modified version of the overall system illumination shown in Fig. 3.1. The only difference is that the objective lens is removed and replaced with a lens of focal length $220 \mathrm{~mm}$ and a variable-diameter iris. This modification produces a beam diameter of $\approx 6 \mathrm{~mm}$ incident on the sample (i.e., the LCVRs). Each LCVR is mounted with its fast axis at an angle of $45^{\circ}$ relative to the $x$-axis of the laboratory reference frame. Additionally, the two LCVRs with temperature control mechanisms were set to $22{ }^{\circ} \mathrm{C}$, while the other two were at room temperature, $\approx 20{ }^{\circ} \mathrm{C}$. Following the LCVR is a vertically oriented analyzer (Newport, 10LP-VIS-B) and an Ophir photodiode detector.

The measurements consist of cycling through the drive voltage of each LCVR 
for a series of LCTF settings. The LCTF cycles from $400 \mathrm{~nm}$ to $720 \mathrm{~nm}$ in increments of $20 \mathrm{~nm}$. At each wavelength, the LCVR cycles from $0-10$ volts in increments of 0.1 volt. A MATLAB routine controls the LCTF, LCVR, and data acquisition.

The calibration of an LCVR is described using the Stokes/Mueller formalism, shown in the following equation:

$$
\mathbf{S}^{\prime}=\mathbf{M}_{\text {analyzer }}\left(k_{1}, k_{2}\right) \mathbf{M}_{w}\left(45^{\circ}, \delta(V)\right) \mathbf{M}_{\mathrm{PBS}}\left(p_{1}, p_{2}\right) \mathbf{S}
$$

where $k_{1}$ and $k_{2}$ are the principal transmittances of the analyzer, $\delta(V)$ is the voltagedependent LCVR retardance, $p_{1}$ and $p_{2}$ are the principal transmittances of the PBS, and $\mathbf{S}=\left[\begin{array}{llll}1 & 0 & 0 & 0\end{array}\right]^{T}$. After dropping the voltage dependence for simplicity, the output Stokes vector is

$$
\mathbf{S}^{\prime}=\left[\begin{array}{c}
\frac{1}{2}\left(k_{1}^{2}+k_{2}^{2}\right)\left(p_{1}^{2}+p_{2}^{2}\right)+\frac{1}{2}\left(k_{1}^{2}-k_{2}^{2}\right)\left(p_{1}^{2}-p_{2}^{2}\right) \cos \delta \\
\frac{1}{2}\left(k_{1}^{2}+k_{2}^{2}\right)\left(p_{1}^{2}-p_{2}^{2}\right)+\frac{1}{2}\left(k_{1}^{2}-k_{2}^{2}\right)\left(p_{1}^{2}+p_{2}^{2}\right) \cos \delta \\
0 \\
p_{1} p_{2}\left(k_{1}^{2}-k_{2}^{2}\right) \sin \delta
\end{array}\right]
$$

and the signal at the detector $S_{D}$ is the first element of $\mathbf{S}^{\prime}$,

$$
S_{D}=\frac{1}{2}\left(k_{1}^{2}+k_{2}^{2}\right)\left(p_{1}^{2}+p_{2}^{2}\right)+\frac{1}{2}\left(k_{1}^{2}-k_{2}^{2}\right)\left(p_{1}^{2}-p_{2}^{2}\right) \cos \delta
$$


Normalization of the measured signal results in the expression

$$
S_{n}=\frac{S_{D}-\min S_{D}}{\max S_{D}-\min S_{D}}=\frac{1}{2}(1-\cos \delta) .
$$

One is then able to compute the retardance as a function of drive voltage,

$$
\delta(V)=\frac{\cos ^{-1}\left(1-2 S_{n}\right)}{2 \pi},
$$

where the factor of $2 \pi$ converts the retardance from radians to waves. The inverse cosine term in equation (3.6) limits the retardance range to the interval $[0,0.5]$ waves. The actual retardance, however, exhibits values outside of this range, so the retardance curve from equation (3.6) requires unwrapping [115]. The unwrapping points at lower drive voltages are sensitive to alignment, temperature, and scatter effects. Figure 3.8 shows the retardance from equation (3.6) before unwrapping for LCVR 3 at $600 \mathrm{~nm}$. Additionally, this figure also shows the sensitivity of the unwrapping points, as the relative maximum at approximately 1.9 volts should have a value of $\delta=0.5$ waves, and the relative minimum at approximately 2.1 volts should have a value of $\delta=0$ waves.

Figure 3.9a shows three unwrapped tuning curves for LCVR 1 at wavelengths of $500 \mathrm{~nm}, 600 \mathrm{~nm}$, and $700 \mathrm{~nm}$, while Fig. 3.9b shows a 2D map of retardance as a function of wavelength and drive voltage for LCVR 1. Due to the low SNR at the shorter wavelengths, tuning curves below $440 \mathrm{~nm}$ cannot be computed. Therefore, 


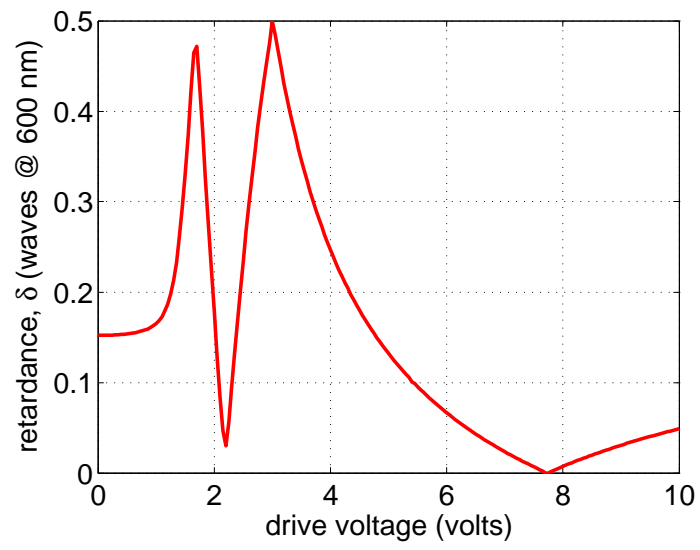

Figure 3.8: Wrapped retardance tuning curve corresponding to equation (3.6) for LCVR 3 at $600 \mathrm{~nm}$.

the spectral range in Fig. $3.9 \mathrm{~b}$ is $440-720 \mathrm{~nm}$. The following three figures, Figs. 3.10, 3.11, and 3.12, show the same data but for LCVRs 2,3 , and 4, respectively.

Figures 3.9-3.12 show the effects of dispersion. In general, dispersion is manifested in these figures as an increase in retardance at the shorter wavelengths for lower drive voltages. For the individual tuning curve figures (Figs. 3.9a-3.12a), dispersion causes the tuning curves acquired at shorter wavelengths to have a larger magnitude, especially in the steep region of the tuning curve. In the 2D maps (Figs 3.9b-3.12b), the spectral dependence produces a slight clockwise rotation, in the plane of the page, of the tuning curve structure.

With the relevant components characterized, the discussion now moves to system assessment. The next section includes a description of the calibration method and techniques for assessing system functionality. 




(a)



(b)

Figure 3.9: a) LCVR 1 tuning curves at three wavelengths (500, 600, and $700 \mathrm{~nm}$ ), b) LCVR 1 tuning curves as a function of wavlength and drive voltage.



(a)

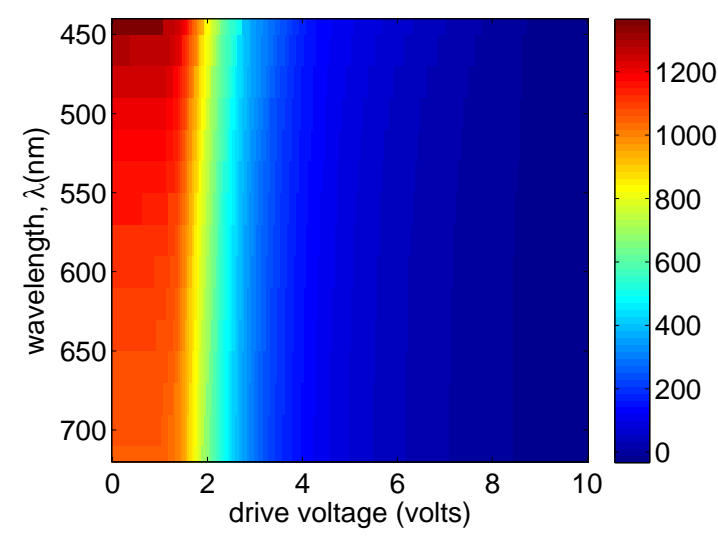

(b)

Figure 3.10: a) LCVR 2 tuning curves at three wavelengths (500, 600, and $700 \mathrm{~nm}$ ), b) LCVR 2 tuning curves as a function of wavlength and drive voltage. 


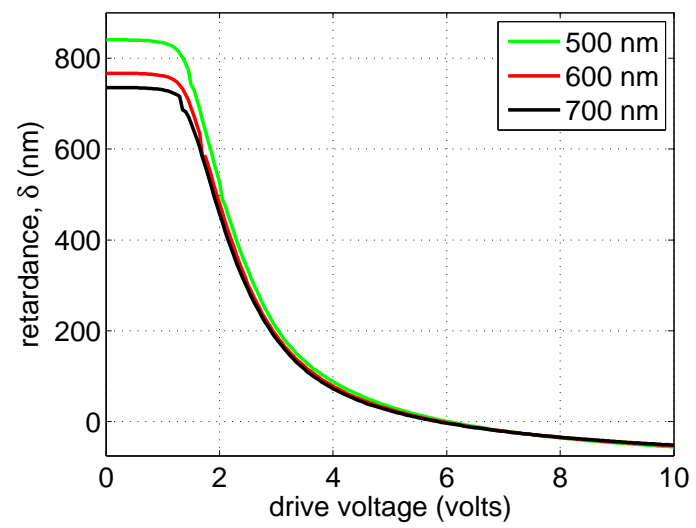

(a)

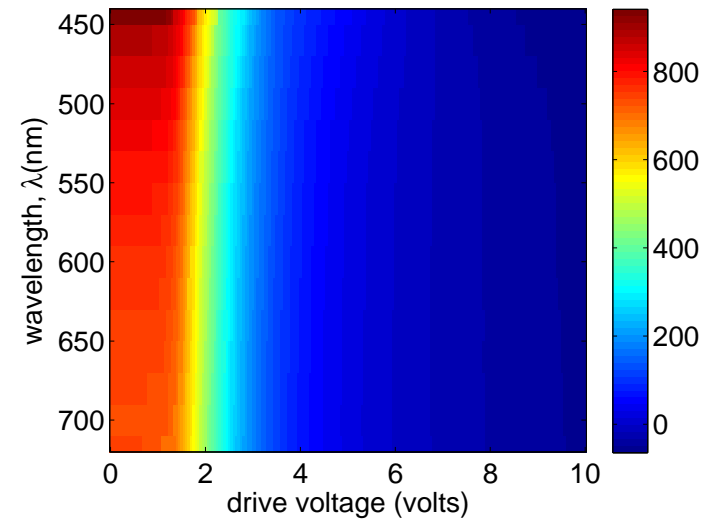

(b)

Figure 3.11: a) LCVR 3 tuning curves at three wavelengths (500, 600, and $700 \mathrm{~nm}$ ), b) LCVR 3 tuning curves as a function of wavlength and drive voltage.

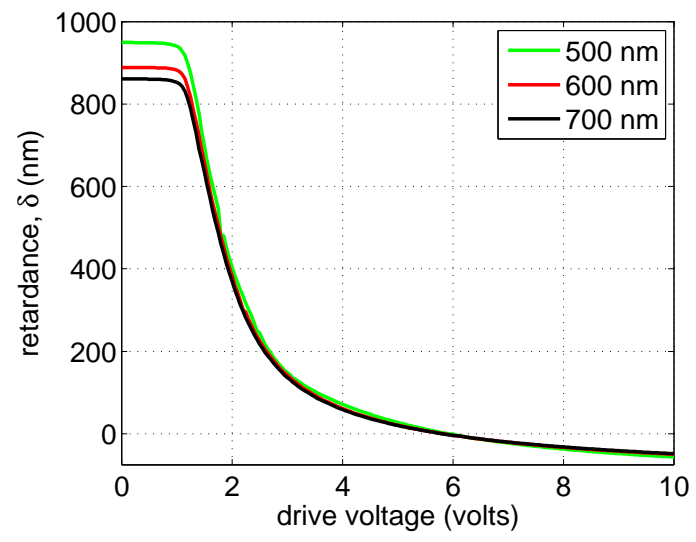

(a)

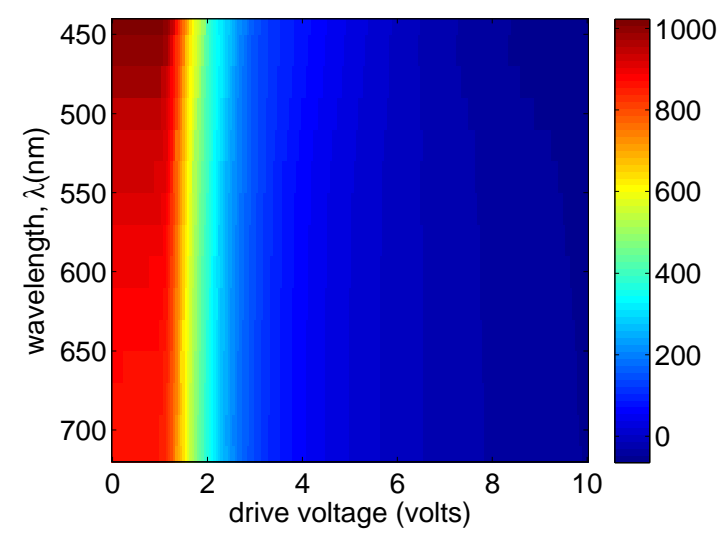

(b)

Figure 3.12: a) LCVR 4 tuning curves at three wavelengths (500, 600, and $700 \mathrm{~nm}$ ), b) LCVR 4 tuning curves as a function of wavlength and drive voltage. 


\subsection{System assessment}

Assessing system performance uses the Stokes/Mueller formalism for providing a quantitative analysis of the performance of the system as a whole. This analysis quantifies several features of the system, including the ability of the system to suppress systematic and random errors. Error suppression is addressed with a theoretical description of the calibration method. Another aspect is the sensitivity of the system to alignment and temperature uncertainties, which is explored through a series of simulations. The final aspect of system assessment is the accuracy and sensitivity of the system when measuring polarization effects. This last aspect is determined through a series of test measurements. The rest of this chapter describes the calibration, simulations, and test measurements.

\subsubsection{Polarimeter calibration}

Accurate Stokes vector estimation requires a properly calibrated polarimeter. Calibration aims to characterize the polarimeter in terms of a data reduction matrix (DRM) W. This data reduction matrix relates the polarimeter measurements I to an estimate of the incident Stokes vector. In principle, it is possible to determine an arbitrary polarization state using four measurements. In this case the data reduction matrix would be of dimension $4 \times 4$. Typically, however, greater accuracy is obtained by performing more than four measurements. In the case of $n$ measurements, the 
data reduction matrix would be of dimension $4 \times n$. Calibration of the polarimeter (calculation of the elements of the data reduction matrix) is performed by presenting a series of known Stokes vectors to the polarimeter stage.

Systems in which the number of measurements exceed the number of unknowns are said to be over-determined. In such cases, the system of equations is solved by calculating the pseudoinverse of the measurement vector. This pseudoinverse is referred to as the least-squares solution. Here, an alternative approach is used employing the singular value decomposition (SVD) [93]. Such an approach is more robust than the least-squares solution in that it accounts for error in the independent and dependent variables (i.e., it is a total least squares solution).

The approach outlined in this section estimates the data reduction matrix without requiring any a priori knowledge about the polarimeter. The process of estimating the DRM, also known as polarimeter training, involves presenting a series of $m$ known Stokes vectors, the training set, to the polarimeter. The polarimeter makes a series of $n$ measurements on each member of the training set. The following equation describes this process:

$$
\mathbf{S}=\mathbf{W I}
$$

where $\mathbf{S}$ is a $4 \times m$ matrix containing the training set, $\mathbf{I}$ is an $n \times m$ measurement vector, and $\mathbf{W}$ is the data reduction matrix of dimension $4 \times n$. If $n>4$, then $\mathbf{W}$ is 
solved for in a least-squares sense,

$$
\hat{\mathbf{W}}=\mathbf{S I}^{T}\left(\mathbf{I I}^{T}\right)^{-1}
$$

where $\mathbf{I}^{T}\left(\mathbf{I I}^{T}\right)^{-1}$ is the pseudoinverse of $\mathbf{W}$. Once $\hat{\mathbf{W}}$ is determined, an unknown Stokes vector can be estimated from subsequent measurements,

$$
\hat{\mathbf{S}}=\hat{\mathbf{W} I}
$$

where $\hat{\mathbf{S}}$ is $4 \times 1, \mathbf{I}$ is $n \times 1$, and $\hat{\mathbf{W}}$ is $4 \times n$.

However, when estimating $\hat{\mathbf{W}}$, the matrix $\mathbf{I I}^{T}$ in equation (3.8) is not wellconditioned and inversion of this matrix can lead to errors in $\hat{\mathbf{W}}$. The condition of a matrix refers to its nearness to singularity and also describes its ease of invertability. Additionally, solving this in a least squares sense is based on the assumption that there is no error in the independent variable. An alternative technique in calculating the pseudoinverse of I entails use of the SVD [93] (See Appendix C). The SVD is a matrix factorization method, often used for solving ill-conditioned inverse problems.

As shown in Appendix C, the measurement matrix $\mathbf{I}$ can be rewritten as

$$
\mathbf{I}=\mathbf{U} \Sigma \mathbf{V}^{T}
$$


where $\mathbf{U}$ is $n \times n, \boldsymbol{\Sigma}$ is $n \times m$, and $\mathbf{V}^{T}$ is $m \times m$. The inverse $\mathbf{I}^{+}$is

$$
\mathbf{I}^{+}=\mathbf{V} \boldsymbol{\Sigma}^{+} \mathbf{U}^{T},
$$

where $\boldsymbol{\Sigma}^{+}$is the inverse of $\boldsymbol{\Sigma}$, and every non-zero diagonal entry in $\boldsymbol{\Sigma}$ is replaced by its reciprocal.

Substituting equation (3.10) into equation (3.7) and rearranging gives an expression for the DRM,

$$
\hat{\mathbf{W}}=\mathbf{S V} \boldsymbol{\Sigma}^{+} \mathbf{U}^{T}=\mathbf{S}_{i} \mathbf{V}\left[\begin{array}{ccc}
\frac{1}{\sigma_{1}} & & \\
& \ddots & \\
& & \\
0 & & \frac{1}{\sigma_{p}}
\end{array}\right] \mathbf{U}^{T}
$$

where $\hat{\mathbf{W}}$ is $4 \times n, \mathbf{S}$ is $4 \times m, \mathbf{V}$ is $m \times m, \boldsymbol{\Sigma}^{+}$is $m \times n, p=\min (m, n), \sigma_{p}$ are the singular values of $\hat{\mathbf{W}}$, and $\mathbf{U}^{T}$ is $n \times n$.

The SVD describes the signal and the noise in the system. This description is in terms of the "instrument" space and the "data" space (the $\mathbf{U}$ and $\mathbf{V}$ matrices in equation (3.10), respectively). The singular values link these two spaces, where the size of the singular value represents the strength of the link. When taking the pseudoinverse of a singular matrix, any zero singular values can have their inverses set to zero. However, small, non-zero eigenvalues that exist outside of the system 
dimension (i.e., the dimension of the Stokes vector) signify noise. These singular values can also have their reciprocals set to zero, which is a technique called truncation. If the small, non-zero singular values are not set to zero, their inverses will be large and will cause error in the pseudoinverse. Truncation enforces a total least squares solution while also reducing the dimensionality of the solution. This in effect gives the solution closest to the data set, yet has the desired dimensions. Because the reference vectors in $\mathbf{S}$ span four dimensions, $\boldsymbol{\Sigma}$ should have a range with only four dimensions. Consequently, the pseudoinverse $\boldsymbol{\Sigma}^{+}$can be truncated and the number of nonzero singular values in $\boldsymbol{\Sigma}^{+}$can be reduced to four,

$$
\hat{\mathbf{W}}=\mathbf{S V} \operatorname{diag}\left(1 / \sigma_{1}, \ldots, 1 / \sigma_{4}, \quad 0, \ldots, 0\right) \mathbf{U}^{T}
$$

where $\hat{\mathbf{W}}$ is $4 \times n, \mathbf{S}$ is $4 \times m, \mathbf{V}$ is $m \times m, \boldsymbol{\Sigma}^{+}$is $m \times n, p=4, \sigma_{p}$ are the singular values of $\hat{\mathbf{W}}$, and $\mathbf{U}^{T}$ is $n \times n$.

Optimizing the data reduction matrix $\mathbf{W}$ results in minimizing its condition number. A minimum condition number indicates that the linear independence of rows and columns is maximized. Tyo [63] proved that the theoretical minimum condition number for $\mathbf{W}$ is

$$
\kappa(\mathbf{W})=(D-1)^{1 / 2}
$$

where $D$ is the dimensionality of the Stokes vector to be reconstructed. Since all four elements of the Stokes vector are desired, $D=4$ and $\kappa(\mathbf{W})=\sqrt{3}$. 
The calibration used here entails making $n=6$ measurements on each incident Stokes vector. By making six measurements, redundancy is introduced and the system of equations is over-determined. This has the effect of making the system less sensitive to noise and error. Recall that these six measurements have been chosen to give the minimum theoretical condition number.

As previously discussed, the $6 \times 1$ measurement vector $\mathbf{I}$ is

$$
\mathbf{I}=\left(\begin{array}{llllll}
I_{H} & I_{V} & I_{+45^{\circ}} & I_{-45^{\circ}} & I_{R C P} & I_{L C P}
\end{array}\right)^{T},
$$

where the components of $\mathbf{I}$ consist of the basic elements of the Stokes vector. For this measurement vector, the DRM can be shown to be [63]

$$
\mathbf{W}=\left[\begin{array}{cccccc}
\frac{1}{3} & \frac{1}{3} & \frac{1}{3} & \frac{1}{3} & \frac{1}{3} & \frac{1}{3} \\
1 & -1 & 0 & 0 & 0 & 0 \\
0 & 0 & 1 & -1 & 0 & 0 \\
0 & 0 & 0 & 0 & 1 & -1
\end{array}\right]
$$

This DRM has a condition number of $\kappa(\mathbf{W})=\sigma_{\max } / \sigma_{\min }=1.414 / 0.817=\sqrt{3}$.

Generating the data reduction matrix typically consists of delivering a set of 36 presumed known reference Stokes vectors to the polarimeter, where the polarimeter makes six measurements on each Stokes vector. Therefore, in the $\mathbf{S}=\mathbf{W I}$ expression used for estimating $\mathbf{W}$, the measurement matrix $\mathbf{I}$ has dimensions of $6 \times 36$ and 
the matrix $\mathbf{S}$ has dimensions of $4 \times 36$. The training set Stokes vectors are labeled as "presumed" due to uncertainty in the alignment and temperature of the Stokes generator LCVRs.

Accurately estimating $\hat{\mathbf{W}}$ requires a training set that spans the entire Poincaré sphere [93]. Spanning the Poincaré sphere exposes the polarimeter to a wide range of reference polarization states, thus ensuring that the polarimeter can reproduce these states from subsequent measurements. The training set consists of two equally-spaced subsets that trace out paths along different great circles: a linearly polarized subset along the Poincaré sphere equator and a primarily elliptically polarized subset that follows a path from pole to pole along a meridian line. The training set is shown in Fig. 3.13, where the red circles correspond to linear Stokes vectors and the blue stars correspond to primarily elliptical Stokes vectors. Although a 36 member training set has also been used in the literature [11,93], this number is somewhat arbitrary, as a rationale for the quantity was not given. The size of the training set is explored in Section 3.4.3.

The Stokes generator LCVRs produce the original 36 member training set from a predetermined set of drive voltages that correspond to specific retardances. Arriving at the drive voltages consists of two main steps. The first step requires the Stokes/Mueller calculus, and involves computing the specific retardances that produce the training set. The computations reveal that varying the retardance of LCVR 1 from $0-1$ waves and fixing the LCVR 2 retardance at either 0.25 waves or 


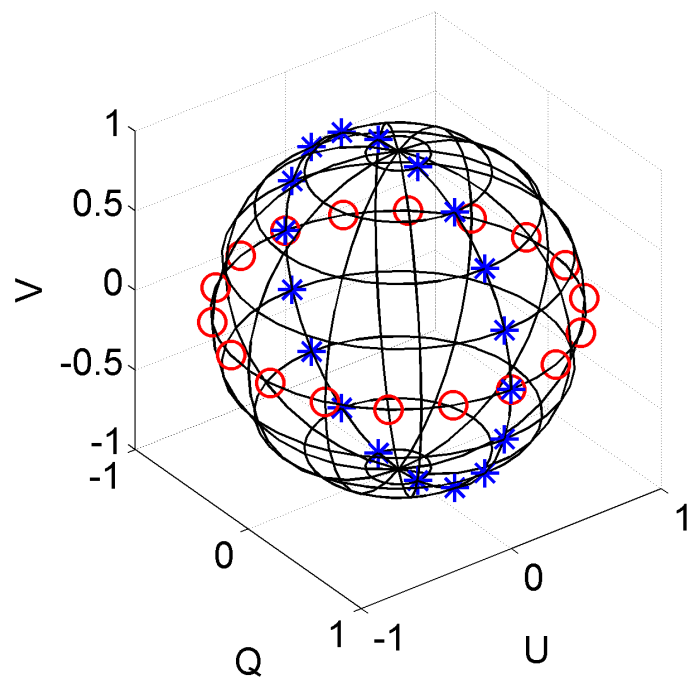

Figure 3.13: Poincaré sphere showing the training set Stokes vectors. The red circles represent linear Stokes vectors, the blue stars represent primarily elliptical Stokes vectors.

0 waves produces the training set. These retardances are summarized in Table 3.1. Lastly, the tuning curves are employed for selecting the drive voltages that produce the desired retardances.

The procedure for determining the polarimeter retardances is the same as that for the Stokes generator retardances. The retardance values corresponding to the six measurements for the pair of LCVRs are shown in Table 3.2. The tuning curves are used for determining the drive voltages that correspond to the desired retardances.

In conclusion, this section described a method for polarimeter calibration that requires no a priori knowledge about the polarimeter components. The method expresses calibration in terms of the data reduction matrix, which is estimated using the SVD. Using the SVD allows for a total least squares solution and the option of 
Table 3.1: Training set retardance values (in waves) for the Stokes generator LCVRs.

\begin{tabular}{|c|c|c|}
\hline & Linear subset & Elliptical subset \\
\hline LCVR $1\left(\mathrm{FA}=45^{\circ}\right)$ & {$[0,1]$} & {$[0,1]$} \\
\hline LCVR $2\left(\mathrm{FA}=0^{\circ}\right)$ & 0.25 & 0 \\
\hline
\end{tabular}

Table 3.2: Measurement retardance values (in waves) for the polarimeter LCVRs.

\begin{tabular}{|c|c|c|c|c|c|c|}
\hline & $\mathrm{H}$ & $\mathrm{V}$ & $+45^{\circ}$ & $-45^{\circ}$ & $\mathrm{RCP}$ & $\mathrm{LCP}$ \\
\hline $\mathrm{LCVR} 3\left(\mathrm{FA}=0^{\circ}\right)$ & 0 & 0 & 0.25 & 0.25 & 0 & 0 \\
\hline LCVR 4 $\left(\mathrm{FA}=45^{\circ}\right)$ & 0.5 & 0 & 0.75 & 0.25 & 0.25 & 0.75 \\
\hline
\end{tabular}

truncation. These two features of the SVD result in the suppression of systematic and random errors. The calibration method can also be used for assessing the propagation of systematic error, and the Mueller matrix sensitivity to these errors. This is the topic of the next section, as system sensitivity to LCVR alignment and temperature effects are explored through a series of simulations.

\subsubsection{LCVR alignment and temperature effects}

It is well known that in liquid crystal-based active polarimetry, alignment and temperature effects impact polarimeter performance. Practically speaking, when constructing a polarimetric measurement system from LCVRs, unavoidable alignment and temperature uncertainties will occur, leading to systematic error that propagates to the Mueller matrix. Typical calibration methods use only a single metric 
to assess polarimeter performance (the condition number) and often ignore the relationship between systematic error and specific Mueller matrix elements. The current section explores alignment and temperature effects in a Stokes generator and polarimeter, each consisting of two LCVRs, through a series of simulations to calibrate the polarimeter and measure the Mueller matrix of air. These effects are explored by modifying an existing LCVR model to incorporate alignment and temperature uncertainties. This new approach offers insight into system performance by associating particular Mueller matrix element error with specific LCVR effects.

Within the large volume of variable retardance polarimetry literature, there is a considerable amount of work regarding optimum polarimeter configurations [63, 90, 91, 95,98] and calibration techniques $[93,94,97,102,116]$. When reporting the effectiveness of a given configuration or calibration, many researchers use the condition number, which quantifies the sensitivity of a system to error. Typical error sources can include random error (i.e., measurement noise) or systematic error (i.e., nonideal optical components, position error, or temperature uncertainty). Implicit in these reports, however, is the assumption that one is able to reliably deliver known polarization states with a Stokes generator in order to calibrate the polarimeter. For example, when implementing data reduction methods, such as in [93], an accurate Stokes generator is assumed. Although the condition number is a valuable metric for assessing system performance in the presence of error, it serves primarily as a general indicator of error magnification and propagation for the given linear system. In other 
words, for a linear system represented by the equation $\mathbf{A x}=\mathbf{b}$, the condition number of $\mathbf{A}$ relates how much errors in $\mathbf{A}$ and/or $\mathbf{b}$ are magnified in the solution vector $\mathbf{x}$.

When performing Mueller matrix polarimetry, the desired result is the complete Mueller matrix. While the condition number aids in assessing the error sensitivity of the system, it does not easily provide error uncertainties for specific elements of the measured Mueller matrix. Although a well-conditioned polarimeter would be expected to produce Mueller matrix measurements with less error than a poorly conditioned polarimeter, the question arises as to what specifically causes particular Mueller matrix element uncertainty. The results here show there exists a relationship between specific Mueller matrix elements and LCVR alignment and temperature uncertainties, and they provide supplemental information beyond the condition number.

LCVRs are computer-controlled birefringent devices filled with a proprietary liquid crystal mixture [109]. They employ a user-controlled drive voltage to adjust device birefringence in order to alter an incident polarization state. The LCVRs used here, manufactured by Meadowlark Optics, use a unique construction for achieving this. A schematic of the LCVR construction is shown in Fig. 3.14. As illustrated in this figure, the LC layer is sealed in a cavity constructed from two glass windows. Each window is also coated with an alignment layer and a current conducting layer, indium tin oxide (ITO). Electrodes are attached to the windows for creating a voltage difference across the cavity, thus establishing the electric field across the LC layer [109]. These devices change the polarization state of light with no mechanical 


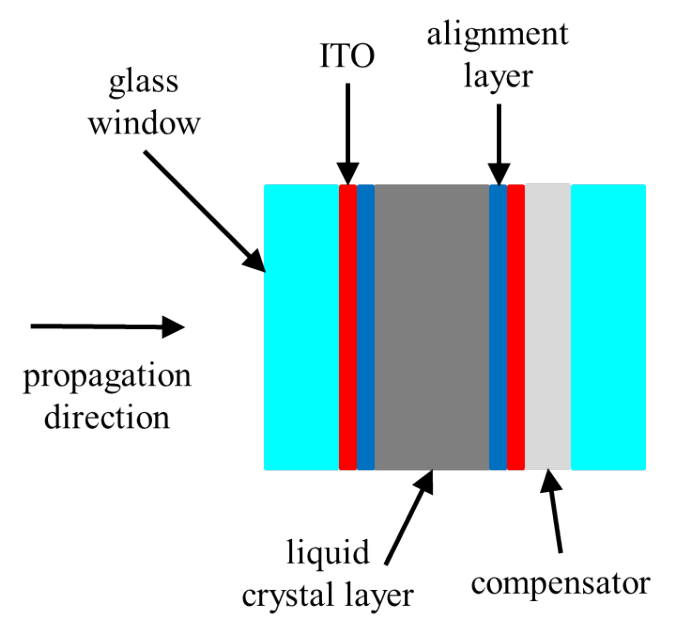

Figure 3.14: Schematic of LCVR construction. Not to scale.

movement by delivering a square wave $\mathrm{AC}$ drive voltage across the LC layer. An AC waveform is necessary for preventing damaging ionic buildup [109]. The voltage causes the LCs to undergo a global, uniform orientation change, thus changing the overall retardance. In order to attain zero retardance, a compensator, which is a fixed polymer retarder, is attached to the LC cell. LCVRs impart spectral retardance effects on a millisecond time scale, where the retardance can vary up to a full wave for a given wavelength. The drive voltage corresponds to a specific retardance as defined by the user-derived tuning curve of the LCVR.

Tuning curves are acquired during a separate calibration, and these curves are sensitive to LCVR alignment and temperature. Great care must be taken when inserting the devices into the Stokes generator or polarimeter, as any deviation from the tuning curve acquisition alignment or temperature will result in systematic error. Consequently, the primary source of systematic error associated with LCVRs is due 
to misalignment (tip, tilt, or both) and temperature, which causes changes to the LC layer birefringence, and hence the tuning curve, of the device.

In this section, alignment and temperature effects are explored in a polarimetric system constructed from four LCVRs, where the Stokes generator and polarimeter each consist of a pair of these devices. This is achieved by simulating misalignments in the Stokes generator LCVRs and temperature uncertainties in both the Stokes generator and polarimeter LCVRs. Polarimeter LCVR misalignment is not explored because the calibration technique used here assumes the systematic error occurs in the Stokes generator. Additionally, the calibration technique establishes a calibration matrix that compensates for small, constant inaccuracies in the polarimeter components. It is also assumed that the temperature uncertainty occurs after calibration but before measurements are made. Each simulation type, either for misalignment or temperature, consists of two main procedures. The first procedure involves polarimeter calibration, and the second procedure simulates Mueller matrix measurements on air. Finally, results include LCVR sensitivity, polarimeter calibration, and Mueller matrix uncertainty.

\section{Theory}

In this section, the Stokes/Mueller calculus is used for describing polarization effects. This formalism describes how a medium interacts with polarized light and can be used for describing optical components, such as retarders. For example, the Mueller 
matrix for a retarder is of the general form

$$
\mathbf{M}(\delta, \theta)=\left[\begin{array}{cccc}
1 & 0 & 0 & 0 \\
0 & a^{2}+c b^{2} & (1-c) a b & -b d \\
0 & (1-c) a b & b^{2}+c a^{2} & a d \\
0 & b d & -a d & c
\end{array}\right],
$$

where $a=\cos 2 \theta, b=\sin 2 \theta, c=\cos \delta, d=\sin \delta, \delta$ is the retardance, and $\theta$ is the fast axis with respect to the $x$-axis of the laboratory coordinate system [19]. The retarder in Fig. 3.14 can be described by the following expression $[111,117]$

$$
\mathbf{M}_{\mathrm{LCVR}}=\mathbf{M}\left(\delta_{C}, \theta_{C}\right) \mathbf{M}\left(\delta_{L C}(V), \theta_{L C}\right),
$$

where $\delta_{C}$ is the constant retardance of the compensator, $\theta_{C}$ is the fast axis of the compensator, $\delta_{L C}(V)$ is the voltage-dependent retardance of the LC layer, and $\theta_{L C}$ is the LCVR fast axis. The compensator fast axis is oriented at $90^{\circ}$ with respect to the nominal LCVR fast axis (i.e., $\theta_{C}=\theta_{L C}+\pi / 2$ ). Additionally, each Mueller matrix in equation (3.18) has the form shown in equation (3.17), with the exception that the LC layer retardance is a function of drive voltage. Therefore, any Mueller matrix element containing the $\delta_{L C}(V)$ term will also be voltage dependent. The retarder description in equation (3.18) allows the incorporation of LCVR alignment and temperature effects. A model developed by Xiao and Voelz, which is used for 
incorporating alignment effects into equation (3.18) [111,117], is discussed in the next two paragraphs. In addition, equation (3.18) and information supplied by the LCVR manufacturer is used for incorporating temperature effects, which is also discussed later in this section.

In order to incorporate tilt and tip angle effects into the retarder Mueller matrix, a variation of the formalism put forth by Xiao and Voelz $[111,117]$ is used. In this development, Xiao and Voelz modeled the voltage-dependent response of an LCVR to rays at oblique incidence angles, where the incident rays were resolved into $x$ and $y$ components in the laboratory coordinate system. This system is a right-handed coordinate system with the positive $z$-axis as the propagation direction. Resolving the rays into their orthogonal components permitted the authors to parameterize the relationship between the incident illumination angle and the voltage-dependent orientation of the LCs in the LC layer. This parameterization allowed them to estimate the modified LC layer retardance as a function of illumination angle $\xi$ and the voltage-dependent LC tilt angle $\beta(V)$. They also found that the fast axis exhibited a dependence on illumination angle and LC tilt angle. Additionally, the retardance of the compensator has a slight dependence on illumination angle. These results were incorporated into the LCVR Mueller matrix of equation (3.18). The modified LCVR Mueller matrix takes the form

$$
\mathbf{M}_{\mathrm{LCVR}}=\mathbf{M}\left[\delta_{C}(\xi), \theta_{C}\right] \mathbf{M}\left[\delta_{L C}(\beta(V), \xi), \theta_{L C}(\beta(V), \xi)\right]
$$


where $\delta_{C}(\xi)$ is the illumination angle-dependent retardance of the compensator, the voltage- and illumination-angle-dependent retardance of the LC layer is $\delta_{L C}(\beta(V), \xi)$, and $\theta_{L C}(\beta(V), \xi)$ is the LCVR fast axis, also dependent on voltage and illumination angle. Both Mueller matrices in equation (3.19) display the form shown in equation (3.17), except that elements containing $\delta_{C}$ are a function of illumination angle, and elements containing $\delta_{L C}$ and $\theta_{L C}$ are a function of LC tilt angle and incident angle.

Here, the formalism is slightly modified to interpret the oblique incidence angles as a tilt or tip of the LCVR in the laboratory coordinate system. A positive tilt is defined as a clockwise rotation about the $y$ axis in the $x$ - $z$ plane, and a positive tip is defined as a clockwise rotation about the $x$-axis in the $y$-z plane (see Fig. 3.15). The modified Xiao formalism is used to alter the LC layer retardance and fast axis based on LCVR tilt or tip. This is necessary, as the tuning curves are obtained from separate LCVR calibration measurements before the LCVRs are relocated to the Stokes generator or polarimeter. This modified formalism results in an LC layer retardance and apparent fast axis orientation based on LCVR tip or tilt, as a function of drive voltage. These parameters, along with the incident-angle dependent compensator retardance, are used in the modified Mueller matrix of equation (3.19).

Incorporating temperature effects consists of modifying equation (3.18) such that it now has the form

$$
\mathbf{M}_{\mathrm{LCVR}}=\mathbf{M}\left[\delta_{C}, \theta_{C}\right] \mathbf{M}\left[\delta_{L C}(\beta(V), T), \theta_{L C}\right],
$$



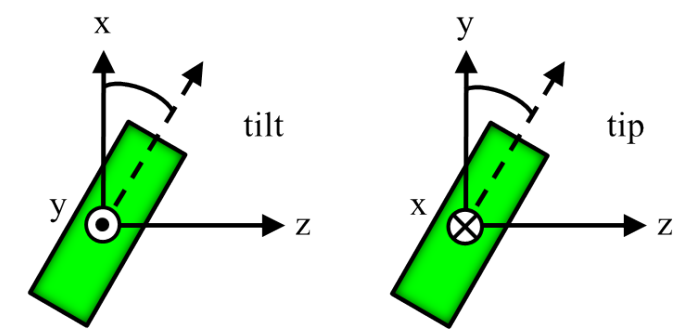

Figure 3.15: Tilt and tip for the LCVRs.

where $\delta_{L C}(\beta(V), T)$ is the LC layer retardance that depends on voltage and temperature. The Mueller matrices in equation (3.20) have the same form as equation (3.17), and elements containing $\delta_{L C}$ are voltage and temperature dependent. The LC layer temperature dependence is specified by the device manufacturer and is discussed in more detail later in this section.

The modified LCVR Mueller matrices serve as important constructs in the polarimeter calibration simulations. Polarimeter calibration establishes a relationship between polarimeter measurements and the incident Stokes vectors. Polarimeter calibration involves using a Stokes generator for presenting a series of known Stokes vectors (i.e., the training set) to the polarimeter and then using the polarimeter for making a series of six intensity measurements on each training set member. The calibration technique is the same technique as that used in Section 3.4.1.

\section{Simulations}

The simulation configuration used here mimics the actual measurement configuration, which consists of a polarization state generator (i.e., Stokes generator) and a detection 


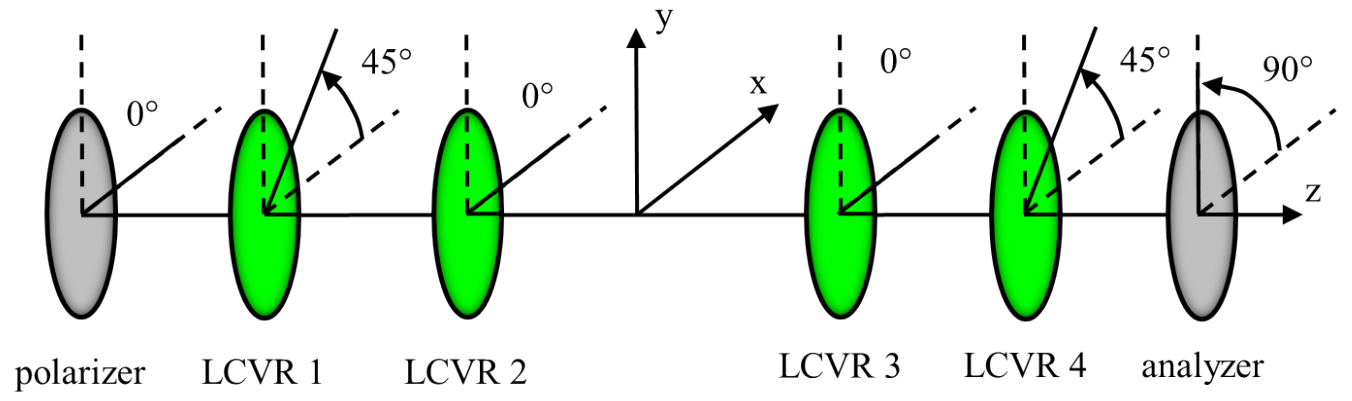

Figure 3.16: Schematic representation of the simulation configuration.

module (see Fig. 3.16). Components used in the Stokes generator include a linear horizontal polarizer, LCVR 1, and LCVR 2 (Meadowlark Optics, LRC-200). LCVR 1 has a fast axis of $45^{\circ}$ with respect to the $x$-axis of the laboratory reference frame, while LCVR 2 has a fast axis of $0^{\circ}$. The detection module contains the polarimeter and consists of LCVRs 3 and 4 (Meadowlark Optics, LRC-200), with fast axes at $0^{\circ}$ and $45^{\circ}$, respectively, and an analyzer oriented at $90^{\circ}$ with respect to the $x$-axis (vertically polarized). Simulations were implemented in MatLaB using the Mueller calculus, previously acquired LCVR tuning curves, and the modified Xiao formalism. The simulations ultimately produced an output Stokes vector that was used for estimating a Mueller matrix. The output Stokes vector $\mathbf{S}^{\prime}$ was computed from the expression

$$
\mathbf{S}^{\prime}=\mathbf{M}_{\text {analyzer }} \mathbf{M}_{\mathrm{LCVR} 4} \mathbf{M}_{\mathrm{LCVR} 3} \mathbf{M}_{\mathrm{LCVR} 2} \mathbf{M}_{\mathrm{LCVR} 1} \mathbf{M}_{\text {polarizer }} \mathbf{S},
$$

where $\mathbf{M}_{\text {analyzer }}$ represents a vertically oriented polarizer, $\mathbf{M}_{\text {polarizer }}$ is a Mueller matrix representing a polarizer oriented horizontally, and $\mathbf{S}=\left[\begin{array}{llll}1 & 0 & 0 & 0\end{array}\right]^{T}$ represents unpolarized incident light. Depending on the effect investigated, only one of the LCVR 
Mueller matrices will have a functional dependence on temperature or alignment, while the other three will not.

The tuning curves for LCVRs 1 and 4 were obtained at normal incidence and $22^{\circ} \mathrm{C}$, as both had temperature control mechanisms. The tuning curves for LCVRs 2 and 3 were obtained at normal incidence and room temperature, $\approx 20{ }^{\circ} \mathrm{C}$. Using the modified Xiao formalism produced modified LC layer retardances, modified apparent fast axis angles, and the resulting Mueller matrices for a number of alignment and temperature uncertainties.

The misalignment simulation consisted of two main procedures. The first procedure involved calibrating the polarimeter with a misaligned Stokes generator, and the second required a measurement on air using the misaligned Stokes generator and calibrated polarimeter. Theoretically, air measurements should produce a Mueller matrix that is the identity matrix, making these results a simple method for assessing polarimeter performance. Additionally, LCVR misalignments were investigated individually, as only one parameter was varied at a time. This meant that the entire simulation was performed for a misalignment in the $x$-direction of LCVR 1 only, then a new simulation for misalignment in the $y$-direction of LCVR 1 only, then the $x$-direction of LCVR 2 only, and then the $y$-direction of LCVR 2 only. Each misalignment simulation was performed for a wavelength range of $400-720 \mathrm{~nm}$ at $20 \mathrm{~nm}$ increments and for 100 different misalignment configurations. Each different individual misalignment configuration from the set of 100 resulted from selecting a 
randomized alignment perturbation for a given LCVR. For example, the first misalignment simulation was the result of 100 alignment perturbations in the $x$-direction of LCVR 1.

Polarimeter calibration was implemented for a training set consisting of $n=$ 36 Stokes vectors. The training set consisted of the same Stokes vectors described previously in Section 3.4.1. The initial step of the calibration procedure was to select a misalignment for a given Stokes generator LCVR. Misalignment angles were drawn from a zero-mean normal distribution with a standard deviation of $\sigma=0.707^{\circ}$. Next, modified versions of the LC layer retardance, compensator retardance, apparent fast axis angle, and LCVR Mueller matrix were computed based on the misalignment. Using the modified LCVR Mueller matrix, calculations were made for determining the perturbed calibration Stokes vectors. The perturbed Stokes vectors were then delivered to the polarimeter. Next, the polarimeter made six measurements on each perturbed Stokes vector. Finally, the data reduction matrix $\mathbf{W}$ and its corresponding condition number were computed.

The second procedure of the misalignment simulation was to measure the Mueller matrix of air. To achieve this, six additional perturbed incident Stokes vectors $\left(\mathrm{H}, \mathrm{V},+45^{\circ},-45^{\circ}, \mathrm{RCP}, \mathrm{LCP}\right)$ were delivered to the polarimeter with the identically misaligned Stokes generator. The polarimeter then made six measurements on each incident Stokes vector, and the previously determined data reduction matrix $\mathbf{W}$ was used for estimating the six Stokes vectors. From the estimated Stokes vectors, the 
Mueller matrix elements were computed by combining specific Stokes vector elements as seen in [10].

As with the alignment simulations, the temperature simulations consisted of two main procedures. First, the polarimeter was calibrated with an aligned Stokes generator using tuning curves at their nominal temperatures. Next, the Mueller matrix measurements were simulated with a given LCVR experiencing a temperature perturbation. This perturbation was a random draw from a zero-mean normal distribution with a standard deviation of $\sigma=0.707^{\circ}$ and was applied only to the LC layer retardance (not the compensator). This was done using information supplied by the manufacturer. According to the manufacturer [109], the overall retardance of a LC cell decreases as temperature increases by approximately $0.4 \%$ per ${ }^{\circ} \mathrm{C}$. Therefore, to simulate temperature effects, the temperature draw was multiplied by both 0.004 and the nominal LC layer retardance and then added to the nominal LC layer retardance as an offset. The new LC layer retardance was then used in the modified LCVR Mueller matrix with the nominal fast axis. The Mueller matrix measurements consisted of 100 different temperature perturbations for the given LCVR. Temperature simulations were done individually for each of the four LCVRs and at the same wavelength range as the misalignment simulations. 


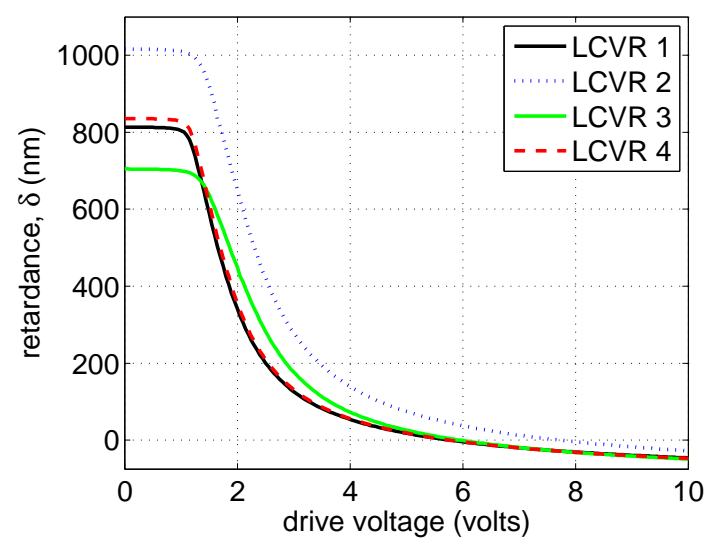

Figure 3.17: User-derived tuning curves for the four LCVRs.

\section{Results}

Figure 3.17 shows the measured tuning curves at normal incidence for the four LCVRs. These tuning curves also serve as the nominal inputs to the simulations and represent the combined effects of the LC layer and the compensator. The tuning curves were obtained by placing each LCVR between crossed polarizers and recording the transmitted intensity as a function of drive voltage. The tuning curve measurements were made using an $852 \mathrm{~nm}$ diode laser. Employment of the Mueller calculus allowed for the computation of retardance from the intensity measurement, and then a phase unwrapping algorithm was applied to the retardance tuning curves. Additional tuning curve measurements were made with intentional misalignments and different temperatures for comparison with the model.

Next, the nominal tuning curve from LCVR 1 was used as the input to the modified Xiao formalism and then compared with results from the two additional 
measurement sets. The first set of measurements were made with LCVR 1 intentionally misaligned and at $22{ }^{\circ} \mathrm{C}$. The second measurement set was made on LCVR 1 at normal incidence but at various temperatures. The intentionally misaligned LCVR measurements were performed at four different tilt angles, denoted as $\gamma_{x}$. Additionally, the temperature measurements were done at four different temperatures for normal illumination. Figure 3.18a shows the normalized intensity transmitted by the LCVR between crossed polarizers as a function of drive voltage for a misalignment of $\gamma_{x}=3^{\circ}$ and the model prediction. Figure 3.18b shows the residual between the model predictions and measurements for a series of tilt angles, including $\gamma_{x}=3^{\circ}$. Figure 3.19a shows the normalized intensity transmitted by the LCVR between crossed polarizers as a function of drive voltage for a temperature of $25^{\circ} \mathrm{C}$ and the model prediction. Figure $3.19 \mathrm{~b}$ shows the residual between the model predictions and measurements for a series of temperatures, including $25{ }^{\circ} \mathrm{C}$. These figures demonstrate that the measurements and modified model are in good agreement.

The modified LC retardance and fast axis data were then used for determining retardance and fast axis sensitivities for LCVRs 1 and 2 relative to misalignment. Sensitivity is defined as

$$
S=\frac{\Delta R / R}{\Delta \gamma / \gamma},
$$

where $R$ is the observable (i.e., the feature of interest) and $\gamma$ is a parameter, either the tilt or tip. LC layer retardance sensitivities for LCVRs 1 and 2 are shown in Fig. 3.20, while fast axis sensitivities are shown for the same devices in Fig. 3.21. The 


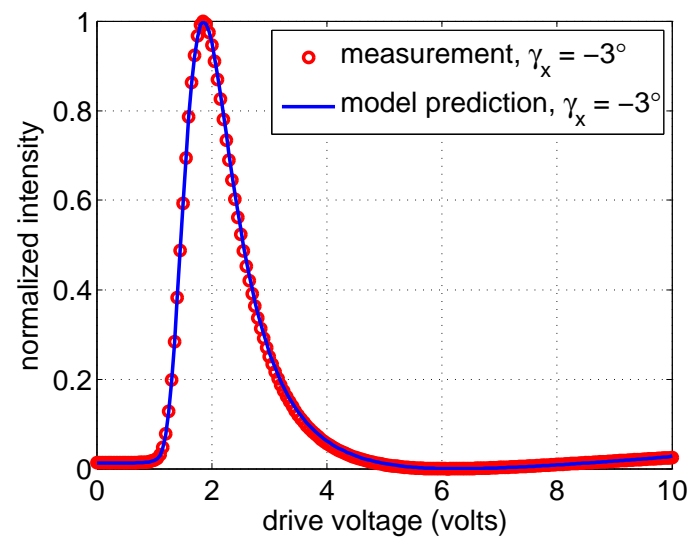

(a)

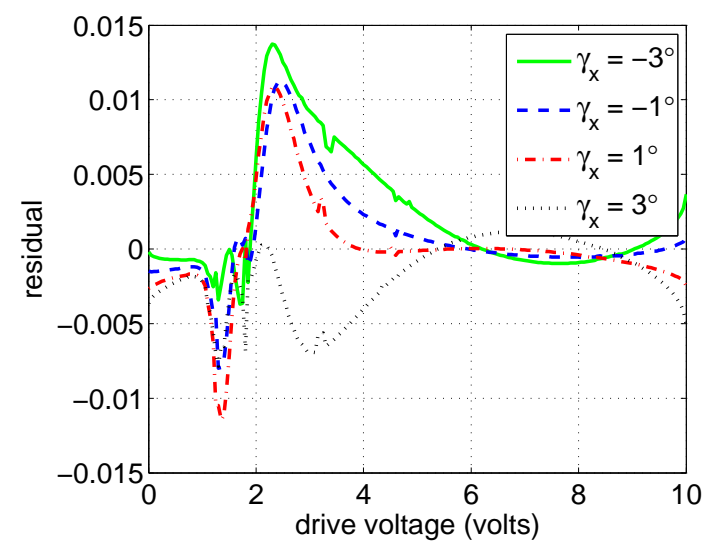

(b)

Figure 3.18: a) Comparison of an intentionally misaligned LCVR measurement and model prediction for LCVR 1. b) Residual between model predictions and measurements for a series of tilt angles.

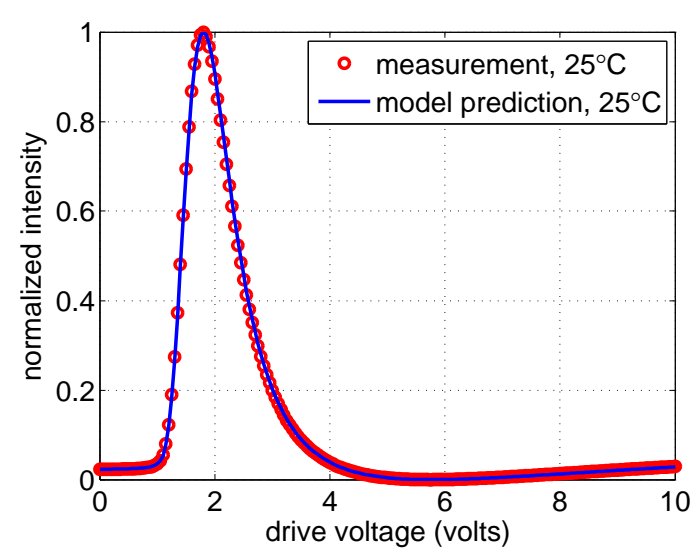

(a)

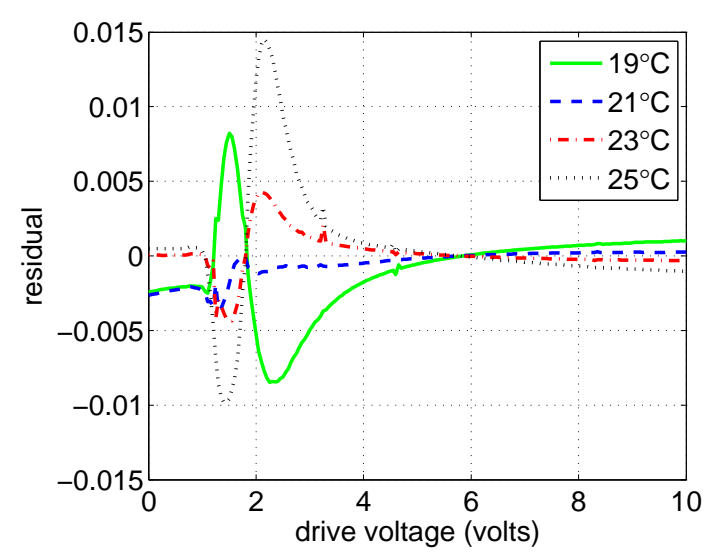

(b)

Figure 3.19: a) Comparison of an LCVR measurement and model prediction for LCVR 1 at a given temperature. b) Residual between model predictions and measurements for a series of temperatures. 


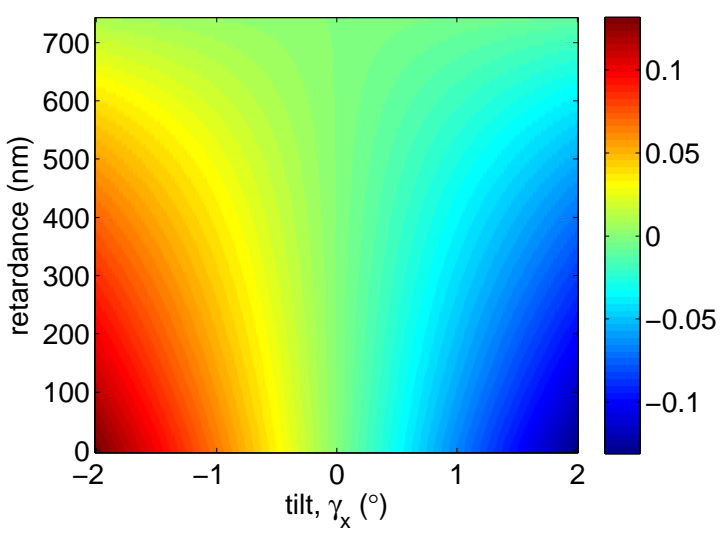

(a) LCVR 1 tilt

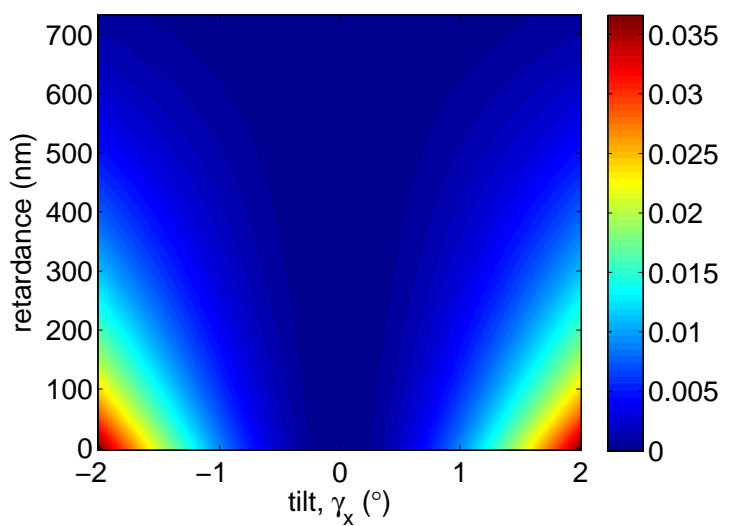

(c) LCVR 2 tilt

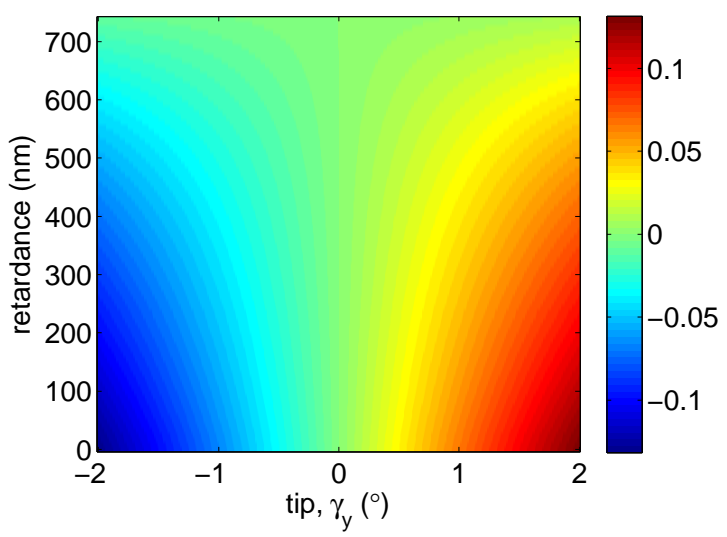

(b) LCVR 1 tip

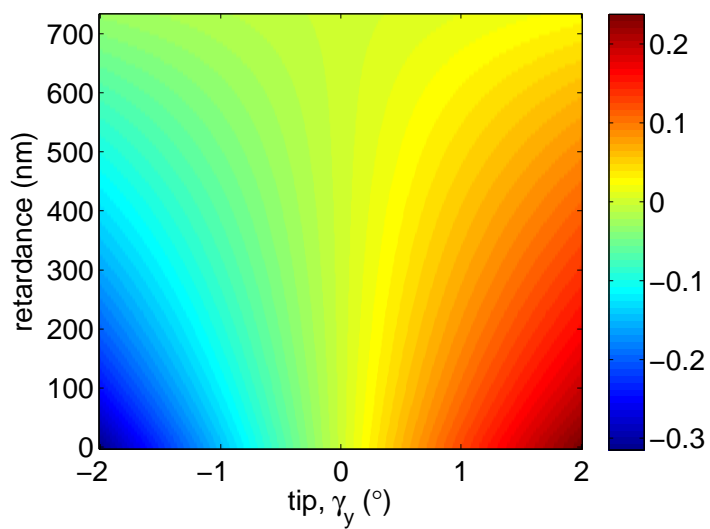

(d) LCVR 2 tip

Figure 3.20: Liquid crystal layer retardance sensitivities for LCVRs 1 and 2.

vertical black line in Fig. 3.21c represents where the fast axis sensitivity is undefined (i.e., $R \rightarrow 0^{\circ}$ in the denominator of the sensitivity equation). Both sensitivities are plotted as a function of tuning curve retardance and angular offset.

The condition number, plotted as the mean and standard deviation as a function of wavelength for each LCVR misalignment simulation, is displayed in Fig. 3.22. Figure 3.23 shows the Mueller matrix uncertainty for the Stokes generator LCVR misalignments. This is expressed as a standard deviation of the zero-mean error 


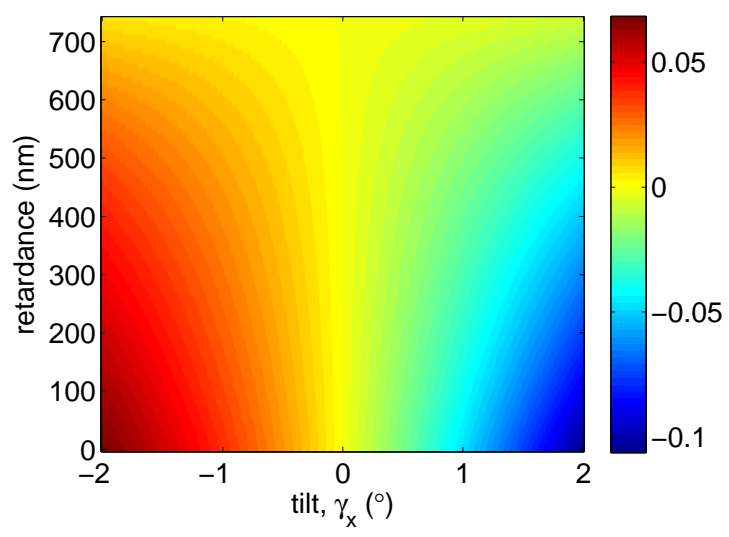

(a) LCVR 1 tilt

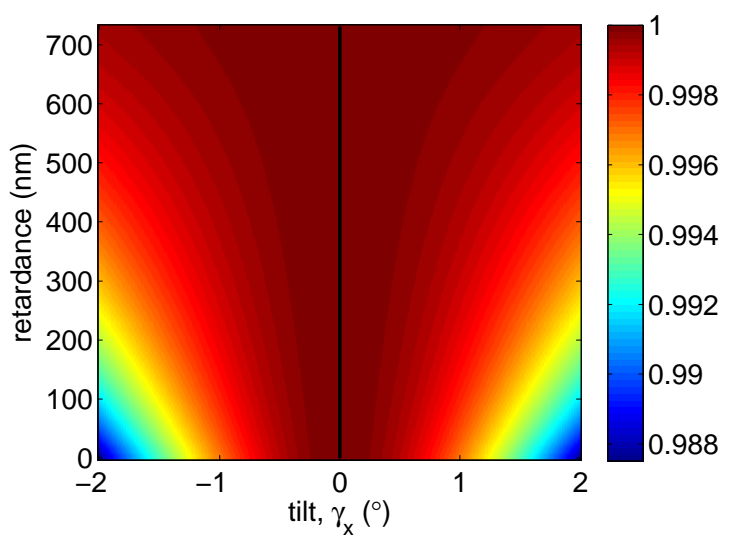

(c) LCVR 2 tilt

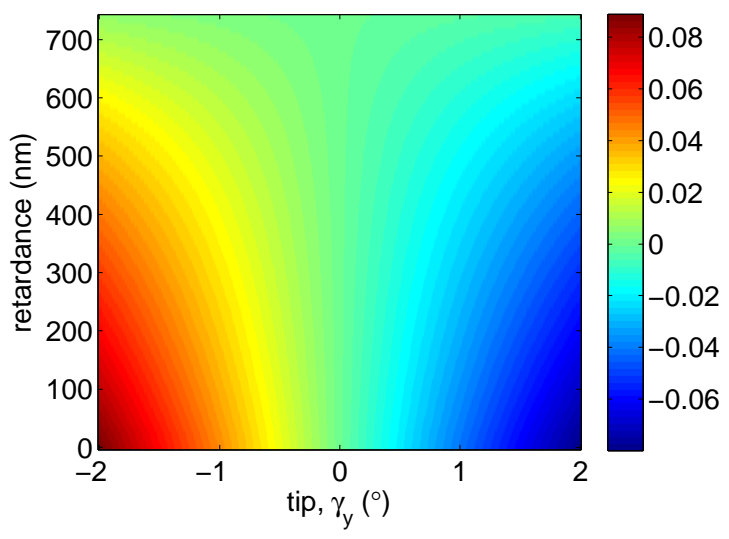

(b) LCVR 1 tip

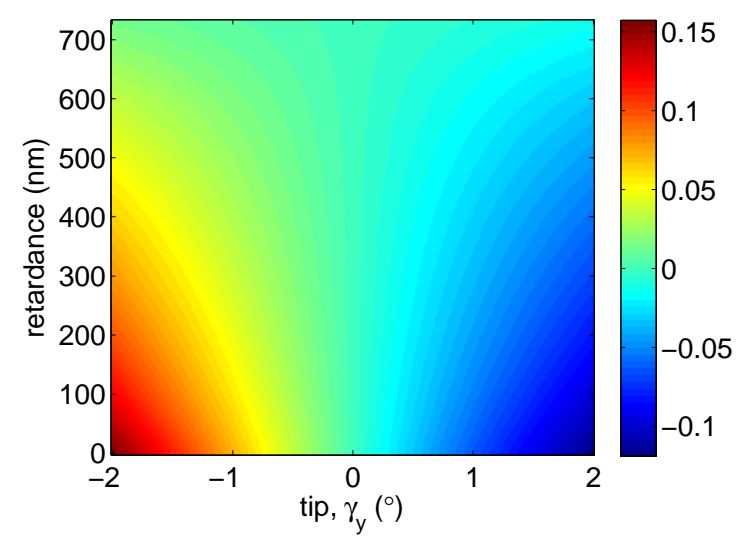

(d) LCVR 2 tip

Figure 3.21: Fast axis sensitivities for LCVRs 1 and 2.

for two wavelengths (400 nm and $720 \mathrm{~nm}$ ) at the four misalignments. The abscissa for each Mueller matrix element represents the four Stokes generator LCVR misalignments $(1 \mathrm{X}, 1 \mathrm{Y}, 2 \mathrm{X}, 2 \mathrm{Y})$. The ordinate shows the standard deviation, which is unitless because the Mueller matrix is normalized. Figure 3.24 shows the Mueller matrix uncertainty for the LCVR temperature perturbations at $400 \mathrm{~nm}$ and $720 \mathrm{~nm}$, where again each element is expressed as the unitless standard deviation. The abscissa of each element represents the four LCVRs in the system. 


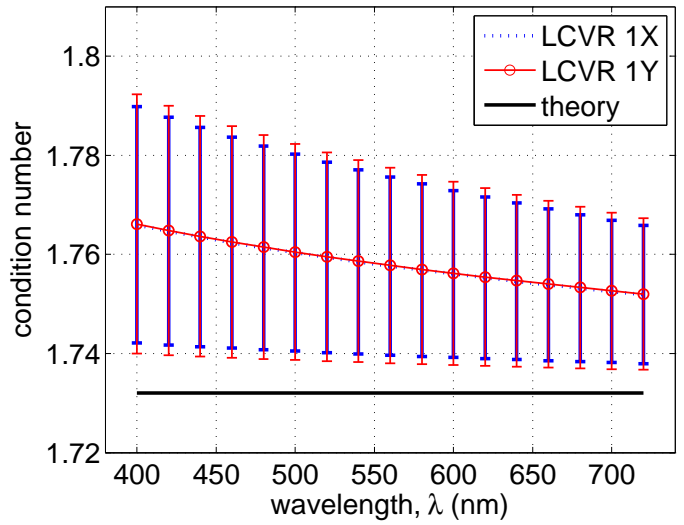

(a) Tilt (x) and tip (y) for LCVR 1

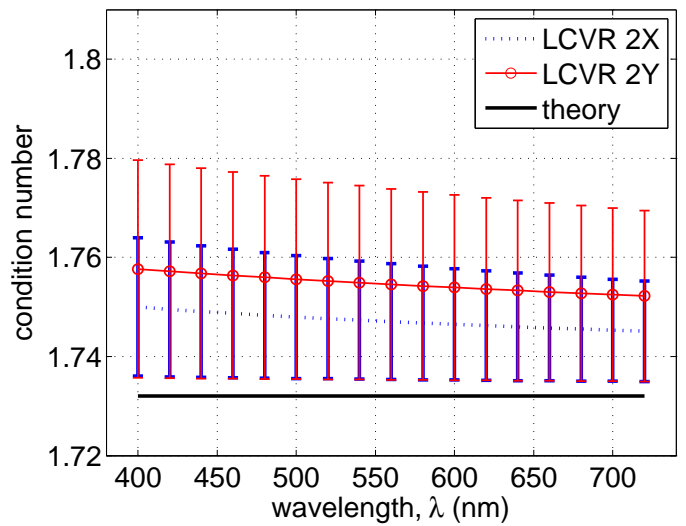

(b) Tilt (x) and tip (y) for LCVR 2

Figure 3.22: Condition number as a function of wavelength for the misalignment configurations. Theoretical minimum is 1.732 , represented by the black line in each plot.
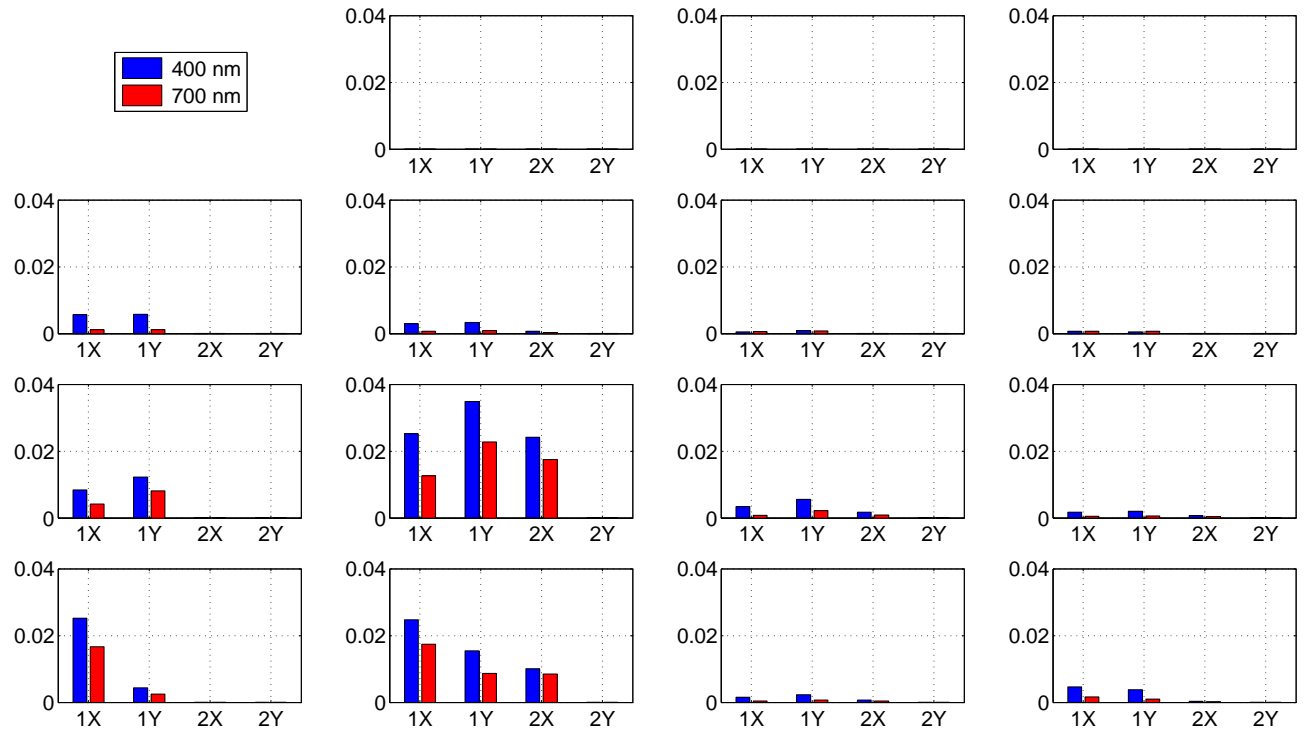

Figure 3.23: Mueller matrix element uncertainty at $400 \mathrm{~nm}$ (blue bars) and 720 $\mathrm{nm}$ (red bars) for the Stokes generator LCVR misalignments. The abscissa for each element represents the four Stokes generator LCVR misalignments (1X, 1Y, 2X, 2Y). The ordinate shows the standard deviation of the zero-mean error, which is unitless because the Mueller matrix is normalized. 

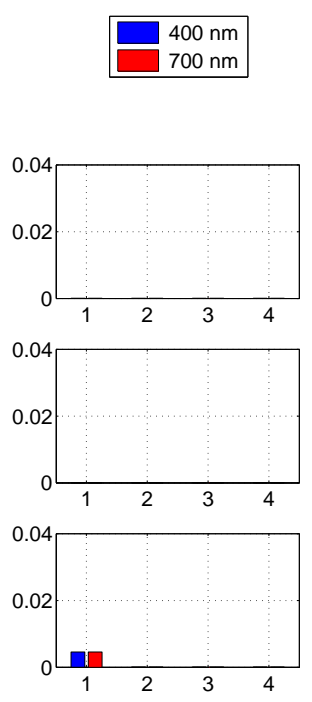
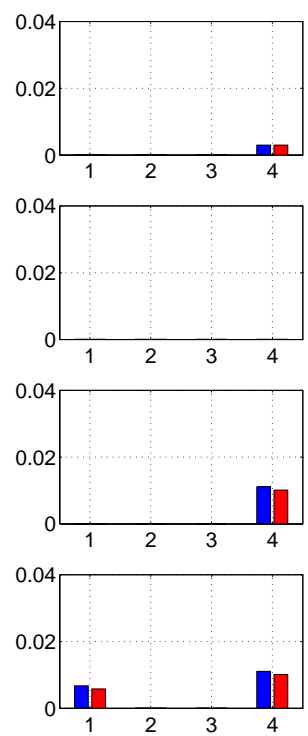
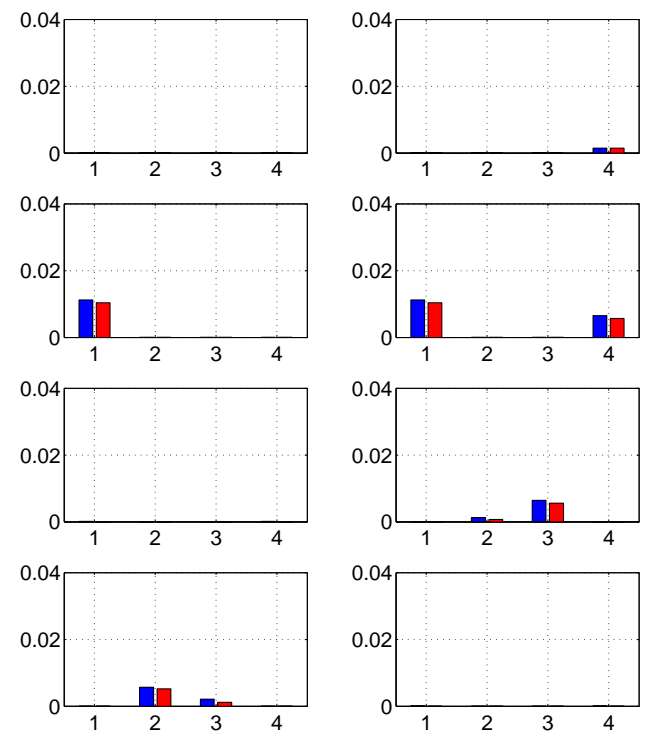

Figure 3.24: Mueller matrix element uncertainty at $400 \mathrm{~nm}$ (blue bars) and $720 \mathrm{~nm}$ (red bars) for the LCVR temperature perturbations. The abscissa of each element represents the four LCVRs in the system $(1,2,3,4)$. The ordinate shows the standard deviation of the zero-mean error, which is unitless because the Mueller matrix is normalized.

\section{Discussion and Conclusions}

The results in Fig. 3.18 show that the modified Xiao formalism accurately describes LCVR misalignment, while Fig. 3.19 shows that using equation (3.18) along with the manufacturers information accurately describes temperature effects. The physical basis of the alignment effects can be explained geometrically. In other words, a misalignment causes changes in the projection of the incident polarization state on to the optical axis of the LCVR, causing the incident light to experience a different LC birefringence and fast axis orientation. On the other hand, the physical basis behind the temperature effects involves thermal expansion of the LC cell. As the 
temperature decreases, the thickness decreases, and the effect of the electric field is stronger. Therefore, the LCs are more apt to align with the electric field. As a result, the LCs exhibit a higher effective birefringence, and hence retardance, at lower temperatures.

The LC retardance and fast axis sensitivity figures (Figs. 3.20 and 3.21) indicate a significant trend. In general, Figs. 3.20 and 3.21 show that there is a higher sensitivity to misalignment at the lower retardances. This appears logical, based on inspection of the simulation data (see Fig. 3.25). Misalignment has the effect of altering the shape of the LC layer retardance curve in a manner that "stretches" it in a particular direction, either in the northeast or southwest direction in the plane of the figure. For example, for the LCVR with a fast axis orientation of $45^{\circ}$, a tilt in the $-x$-direction $\left(-2^{\circ}\right.$ in Fig. 3.25$)$ stretches the LC layer retardance northeast, while a tilt in the $+x$-direction $\left(2^{\circ}\right.$ in Fig. 3.25$)$ causes a deformation toward the southwest. However, in the $x$-direction for the LCVR oriented at $0^{\circ}$, the LC layer retardance undergoes a minimal deformation, as evidenced by the small sensitivity values in Fig. 3.20c. For both LCVR fast axis orientations, a tip in the $-y$-direction causes a southwest stretch, while a tip in the $+y$-direction causes a northeast stretch. These deformations influence the flatter region of the LC retardance more so than the steeper region. The flatter region corresponds to smaller retardances, so at shorter wavelengths, the effect is exacerbated. As a result, shorter wavelengths display higher sensitivity to LCVR misalignment than longer wavelengths. This phenomenon is also 


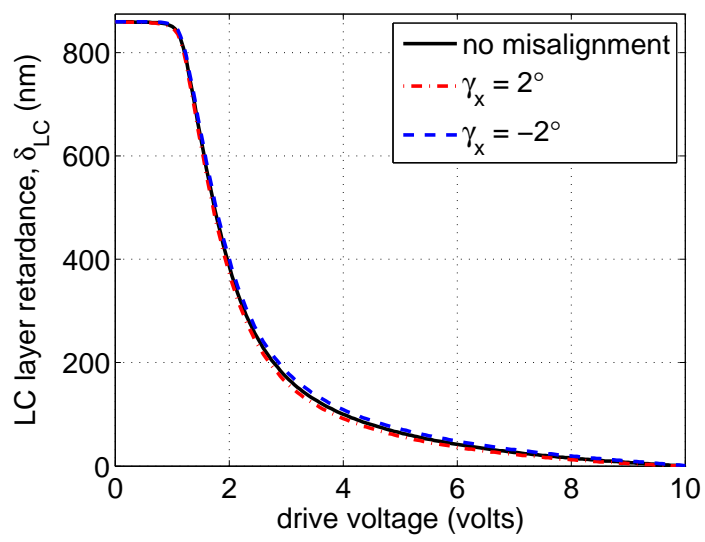

Figure 3.25: Simulation data illustrating the "stretching" effect of the LC layer retardance curve caused by misalignment.

evident in Figs. 3.22-3.24, as the error decreases with increasing wavelength.

The LC retardance and fast axis sensitivities of LCVR 1 have a magnitude of $\approx 0.1$ for both tilt and tip at large misalignments. However, LCVR 2 exhibits different sensitivities for a tilt and tip, as it has a nominal fast axis of $0^{\circ}$. This orientation causes LCVR 2 to display a high LC retardance sensitivity in the $y$-direction at large misalignments (magnitude of $\approx 0.3$ for a tip) and a fast axis sensitivity in the $x$-direction (magnitude of $\approx 1$ for a tilt). LCVR 2 shows a relatively small LC retardance sensitivity to misalignment in the $x$-direction (magnitude of $\approx 0.04$ for a tilt) and a fast axis sensitivity magnitude in the $y$-direction of $\approx 0.15$.

Figure 3.22 shows the spectral dependence of the mean condition number, along with error bars that represent the standard deviation. This figure indicates the sensitivity of the condition number to LCVR misalignment. The larger error bars at the shorter wavelengths reflect an increased sensitivity to misalignment. 
The Mueller matrix uncertainties in Fig. 3.23 also demonstrate the tendency for greater uncertainty at shorter wavelengths, as in general there is larger error in the $400 \mathrm{~nm}$ Mueller matrix entries compared to the $720 \mathrm{~nm}$ entries. Additionally, these figures show how a specific LCVR misalignment can impact individual Mueller matrix elements. For example, if LCVR 1 had a tilt, tip, or both, then there would be significant Mueller matrix error in rows 3 and 4 in the first and second column. Additionally, if there were only a tilt in LCVR 2, then error would exist in elements $(3,2)$ and $(4,2)$.

The temperature perturbation simulations in Fig. 3.24 show how specific LCVR temperature uncertainties map to specific Mueller matrix elements. Figure 3.24 shows that if there is a temperature perturbation associated with LCVR 1 , then Mueller matrix elements $(2,3),(2,4),(4,1)$, and $(4,2)$ would have error. For the case of LCVR 2, the $(4,3)$ element would have error. Temperature perturbations for LCVR 3 would produce error in element $(3,4)$. For the case of LCVR 4, error can be seen in elements $(2,4),(3,2)$, and $(4,2)$, with small error in $(1,2)$ and $(1,4)$.

In addition, some of the Mueller matrix element sensitivities are mutually exclusive. For example, error in the $(2,3)$ element would indicate temperature uncertainty in LCVR 1 only. The same could be said for elements $(4,3)$ and $(3,4)$ for LCVRs 2 and 3, respectively. Also, if elements $(2,1),(2,2),(3,3)$, and $(4,4)$ had error at shorter wavelengths, that would most likely be exclusive to some type of misalignment in LCVR 1. 
The results shown here have significant implications for design and implementation. For example, the sensitivity results show that LCVRs at different orientations have unique responses to misalignment. LCVRs are often configured with their fast axes in positions other than the nominal positions used here (i.e., $\approx 22^{\circ}$ in some polarimeter configurations [11]). The sensitivity simulations provide insight into the consequences for imprecise alignment at various orientations. Additionally, the sensitivity results indicate regions of the tuning curve that are less reliable, such as the portion corresponding to lower retardances. These results show that misalignment influences incident light at shorter wavelengths more so than longer wavelengths. In a laboratory setting, this observation could go unnoticed because optical components specified for the visible spectrum typically exhibit poorer signal-to-noise ratios (SNRs) at the shorter wavelengths. Thus, the poorer performance at shorter wavelengths could be mistakenly attributed to SNR effects rather than a combination of SNR and misalignment effects. It should be noted, however, that the model does not account for wavelength dependence, even though the LCVRs exhibit dispersion. The amount of dispersion is small and did not have any impact on the study. In order to determine spectrally dependent retardances, the retardance of interest is scaled based on the given wavelength, and an interpolation is done on the tuning curve for determining the drive voltage. For example, if a retardance of one half-wave for a wavelength of $600 \mathrm{~nm}$ is required, then an interpolation is done on the tuning curve for determining the drive voltage for $300 \mathrm{~nm}$. 
The sensitivity results can also be used for determining the most effective region of the tuning curve to employ. For example, when selecting the retardances for the training set Stokes vectors, it would be advantageous to select retardances from the steeper portion of the tuning curve. That is, if a particular incident Stokes vector at $600 \mathrm{~nm}$ required LCVR 2 of the Stokes generator to have a retardance of 0 radians ( $0 \mathrm{~nm}$ on the tuning curve), then it would be favorable to use a retardance of $2 \pi$ radians (600 $\mathrm{nm}$ on the tuning curve) instead. The same concept could be applied to the polarimeter LCVRs, thus forming a more effective measurement strategy.

Although the individual sensitivities suggest specific responses to misalignment, the Mueller matrix results suggest otherwise. In other words, the architecture effect is different than the individual effect. For example, because LCVR 2 has the largest individual misalignment sensitivities, one would expect misalignments for LCVR 2 to have a significant effect on the Mueller matrix uncertainties. However, that is not the case for the measurement architecture given here, as misalignments associated with LCVR 1 have a larger impact on Mueller matrix uncertainty. Therefore, all of the available calibration information must be taken into consideration when investigating misalignment effects.

Finally, this analysis also could be useful for establishing a protocol for temperature control. For example, the results here show which LCVRs require precise temperature control. According to the results, LCVRs 1 and 4 exhibit the highest temperature sensitivities, and in multiple Mueller matrix elements, suggesting their 
temperatures should be monitored closely. If individual LCVR temperature control was not available, the results could be used for determining the level to which ambient temperature fluctuations are controlled. Additionally, if there are ambient temperature fluctuations and no LCVR temperature control mechanisms, this analysis could be useful in determining a threshold point for when a recalibration should be performed. Additionally, the results suggest that if there is no temperature control on LCVRs 2 and 3, then as long as the ambient room temperature only fluctuates by $\approx \pm 1^{\circ} \mathrm{C}$, there should not be significant error in the Mueller matrix elements.

To conclude this section, results have been presented from a series of simulations in which Stokes generator LCVR misalignments were modeled during polarimeter calibration and measurements, along with simulations incorporating temperature perturbations to all LCVRs. Simulation results were used for providing an LCVR sensitivity analysis, calibration assessment, and an estimation of Mueller matrix uncertainty. This analysis could be helpful in identifying the sources of systematic error due to alignment or temperature effects in a polarization measurement system consisting of LCVRs.

The next section extends the simulation procedure for investigating additional effects. These effects include different tuning curve regions for suppressing alignment and temperature uncertainty, arbitrary training sets, and training set size. 


\subsubsection{Performance analysis}

The next step in the system assessment is implementing a performance analysis. Performance analysis includes determining how the LCVR alignment and temperature effects impact both the Stokes generator and polarimeter, and then determining an effective strategy for minimizing the systematic error caused by these effects. Strategies include determining the most reliable tuning curve region for the desired LCVR setting for both the Stokes generator and polarimeter LCVRs, selecting the training set Stokes vectors, and determining the proper size of the training set. These strategies are presented in the context of system calibration, as the strategies can be assessed with the simulation procedure described in the previous section. The current section begins with a summary of LCVR effects and their impact, and ends with a description of the various strategies and their results.

\section{LCVR effects and their impact}

LCVR alignment and temperature effects were discussed in the previous section. There it was shown that misalignments impact only the Stokes generator, while temperature variations impact all LCVRs. In reference to alignment effects, the calibration technique compensates for any misalignments in the polarimeter LCVRs, and assumes any systematic error is present in the Stokes generator. Thus, any attempt 
at minimizing alignment effects should focus on the Stokes generator. For temperature effects, it is assumed that the system is calibrated at a given temperature, but then the ambient temperature fluctuates after calibration but before subsequent measurements. This impacts all devices, so any technique for minimizing temperature effects should be investigated for both the Stokes generator and polarimeter. Additionally, LCVRs exhibit greater sensitivity to misalignments and temperature variations at shorter wavelengths (i.e., tuning curve regions with lower retardances at higher drive voltages). Thus, mitigating these error sources could be achieved by using higher retardance values (i.e., lower drive voltages).

\section{Exploring various tuning curve regions}

Selecting the proper LCVR settings for the entire system involves determining the tuning curve regions that minimize LCVR alignment and temperature effects. In order to determine this, simulations for exploring these effects were performed at various Stokes generator and polarimeter settings. The device settings were varied such that they emphasized particular tuning curve regions. In other words, if a value of 0 waves ( 0 radians) was desired, it was possible to use a value of 1 wave ( $2 \pi$ radians) instead. Also, some values of 0.25 waves ( $\pi / 2$ radians) were replaced with 0.75 waves $(3 \pi / 2$ radians $)$.

Changing the device settings in the aforementioned manner produced four different simulation configurations. The first configuration uses Stokes generator and 
polarimeter values of 0 waves and 0.25 waves. These values are also referred to as the nominal retardance values, as these values were introduced in Section 3.4.1. Table 3.3 shows the range of nominal values for the training set Stokes vectors, where the retardance of LCVR 1 spans values including 0 , but not 1 . Table 3.4 shows the six nominal incident Stokes vectors for generating the Mueller matrix, while Table 3.5 shows the nominal polarimeter measurement values. This combination of low retardance values in both the Stokes generator and polarimeter is referred to as the low/low configuration.

Next, the second configuration uses higher values of 1 wave and 0.75 waves. For this case, all values of 0 wave retardance are changed to 1 wave. Additionally, for the training set, the LCVR 1 retardance values span a range that includes 1 wave, and the LCVR 2 retardance is changed from 0.25 to 0.75 waves. For the $+45^{\circ}$ linearly polarized incident Stokes vector, both LCVRs 1 and 2 can be changed to 0.75 waves, and the polarimeter measurement for $-45^{\circ}$ linear polarization can be changed to 0.75 waves for both LCVRs. With high retardance values in both the Stokes generator and polarimeter, this configuration is called the high/high configuration. Tables 3.6, 3.7, and 3.8 show these values, where the yellow boxes highlight values that have changed relative to the low/low configuration.

The final two sets of retardance values use variations of the original two sets. The third set uses lower retardances in the Stokes generator (i.e., 0 and 0.25 waves) and higher retardances in the polarimeter (i.e., 1 and 0.75 waves), and is the low/high 
configuration. Alternatively, the fourth set uses higher retardances in the Stokes generator (i.e., 1 and 0.75 waves) and lower retardances in the polarimeter (i.e., 0 and 0.25 waves), and it is the high/low configuration.

The four configurations were used in the same simulations outlined in the previous section. To review, the previous section described a procedure for simulating misalignments in the Stokes generator LCVRs, and temperature perturbations in all four LCVRs in the system. Thus, each of the four configurations (which correspond to specific device settings) were used for exploring the minimization of both alignment and temperature effects. 
Table 3.3: Nominal training set retardance values (in waves).

\begin{tabular}{|c|c|c|}
\hline & Linear subset & Elliptical subset \\
\hline LCVR 1 $\left(\mathrm{FA}=45^{\circ}\right)$ & {$[0,1)$} & {$[0,1)$} \\
\hline LCVR 2 $\left(\mathrm{FA}=0^{\circ}\right)$ & 0.25 & 0 \\
\hline
\end{tabular}

Table 3.4: Nominal Stokes generator retardance values (in waves).

\begin{tabular}{|c|c|c|c|c|c|c|}
\hline & $\mathrm{H}$ & $\mathrm{V}$ & $+45^{\circ}$ & $-45^{\circ}$ & $\mathrm{RCP}$ & $\mathrm{LCP}$ \\
\hline LCVR 1 $\left(\mathrm{FA}=45^{\circ}\right)$ & 0 & 0.5 & 0.25 & 0.75 & 0.25 & 0.75 \\
\hline LCVR 2 $\left(\mathrm{FA}=0^{\circ}\right)$ & 0 & 0 & 0.25 & 0.25 & 0 & 0 \\
\hline
\end{tabular}

Table 3.5: Nominal retardance values (in waves) for the polarimeter measurements.

\begin{tabular}{|c|c|c|c|c|c|c|}
\hline & $\mathrm{H}$ & $\mathrm{V}$ & $+45^{\circ}$ & $-45^{\circ}$ & $\mathrm{RCP}$ & $\mathrm{LCP}$ \\
\hline LCVR 3 $\left(\mathrm{FA}=0^{\circ}\right)$ & 0 & 0 & 0.25 & 0.25 & 0 & 0 \\
\hline LCVR 4 $\left(\mathrm{FA}=45^{\circ}\right)$ & 0.5 & 0 & 0.75 & 0.25 & 0.25 & 0.75 \\
\hline
\end{tabular}

Table 3.6: High retardance values (in waves) for the training set.

\begin{tabular}{|c|c|c|}
\hline & Linear subset & Elliptical subset \\
\hline LCVR 1 $\left(\mathrm{FA}=45^{\circ}\right)$ & $(0,1]$ & $(0,1]$ \\
\hline LCVR 2 $\left(\mathrm{FA}=0^{\circ}\right)$ & 0.75 & 1 \\
\hline
\end{tabular}

Table 3.7: High retardance values (in waves) for the Stokes generator.

\begin{tabular}{|c|c|c|c|c|c|c|}
\hline & $\mathrm{H}$ & $\mathrm{V}$ & $+45^{\circ}$ & $-45^{\circ}$ & $\mathrm{RCP}$ & $\mathrm{LCP}$ \\
\hline LCVR 1 $\left(\mathrm{FA}=45^{\circ}\right)$ & 1 & 0.5 & 0.75 & 0.75 & 0.25 & 0.75 \\
\hline LCVR 2 $\left(\mathrm{FA}=0^{\circ}\right)$ & 1 & 1 & 0.75 & 0.25 & 1 & 1 \\
\hline
\end{tabular}

Table 3.8: High retardance values (in waves) for the polarimeter measurements.

\begin{tabular}{|c|c|c|c|c|c|c|}
\hline & $\mathrm{H}$ & $\mathrm{V}$ & $+45^{\circ}$ & $-45^{\circ}$ & $\mathrm{RCP}$ & $\mathrm{LCP}$ \\
\hline LCVR 3 $\left(\mathrm{FA}=0^{\circ}\right)$ & 1 & 1 & 0.25 & 0.75 & 1 & 1 \\
\hline LCVR 4 $\left(\mathrm{FA}=45^{\circ}\right)$ & 0.5 & 1 & 0.75 & 0.75 & 0.25 & 0.75 \\
\hline
\end{tabular}




\section{Arbitrary training sets}

Historically, the training set was selected systematically. This was due to limitations in the mechanical repositioning of the components in the Stokes generator. For example, the Stokes generator has normally been a combination of a quarter-wave plate and a rotating or moving polarizer. This type of Stokes generator can access any point on the Poincaré sphere and can generate training sets that span the sphere (i.e., training sets that cover the sphere with balanced polarization content). However, mechanical repositioning can be time-consuming and can introduce systematic error. A Stokes generator consisting of a pair of LCVRs can rapidly access any point on the Poincaré sphere, offering the ability for exploring arbitrarily selected Stokes vectors. An arbitrary training set could mitigate the effects of systematic error, but might not necessarily span the entire sphere, as an arbitrary set does not guarantee adequate polarization diversity. This section investigates the effects of an arbitrary training set as an alternative method to the traditional systematic set. The rest of this section describes the theory for generating arbitrary Stokes vectors and for parameterizing Poincaré sphere coverage, along with a technique for performing calibration simulations with an arbitrary training set.

Generating arbitrary Stokes vectors can be visualized as randomly selecting points on a unit sphere in the spherical coordinate system. In this coordinate system, the spherical coordinates have domains $\theta \in[0,2 \pi)$ and $\phi \in[0, \pi)$. The first step 
in this generation process involves selecting the random variables $V$ and $W$ from a uniform distribution between 0 and 1 to give the values $v$ and $w$. These values map to the spherical coordinates $\theta$ and $\phi$ through the following expressions [118],

$$
\begin{aligned}
& V \sim U(0,1) \quad \Rightarrow \quad \theta=2 \pi v \\
& W \sim U(0,1) \quad \Rightarrow \quad \phi=\cos ^{-1}(2 w-1) .
\end{aligned}
$$

These expressions ensure that small areas on the sphere contain the same point densities [118]. Next, the spherical coordinates are converted to Cartesian coordinates,

$$
\begin{aligned}
& x=\cos \theta \sin \phi \\
& y=\sin \theta \sin \phi \\
& z=\cos \phi .
\end{aligned}
$$

The Cartesian coordinates are then mapped onto the Poincaré sphere, but in terms of the Mueller calculus for the Stokes generator. The $x, y$, and $z$ coordinates correspond to the $Q, U$, and $V$ elements of the Stokes vector. Using the Mueller calculus, the Stokes vector components can be expressed as a function of LCVR 
retardance. This expression is

$$
\left[\begin{array}{l}
I \\
x \\
y \\
z
\end{array}\right]=\left[\begin{array}{c}
I \\
Q \\
U \\
V
\end{array}\right]=\left[\begin{array}{c}
1 \\
\cos \delta_{1} \\
\sin \delta_{1} \sin \delta_{2} \\
\sin \delta_{1} \cos \delta_{2}
\end{array}\right]
$$

where $\delta_{1} \in[0,2 \pi)$ is the retardance for $\operatorname{LCVR} 1$, and $\delta_{2} \in[0, \pi)$ is the retardance for LCVR 2. The retardances that correspond to the Cartesian coordinates are found by rearranging equation (3.25) so that

$$
\begin{aligned}
& \delta_{1}=\cos ^{-1} x \\
& \delta_{2}=\cos ^{-1}\left(\frac{z}{\sin \delta_{1}}\right) .
\end{aligned}
$$

The Stokes vector elements from equation (3.25) can also be used for parameterizing the Poincaré sphere coverage of the training set. This parameterization consists of computing the average arc distance along the Poincaré sphere surface between all pair-wise training set Stokes vectors. For a given pair of Stokes vectors, the arc distance $d$ is computed from [119]

$$
d_{i j}=\cos ^{-1}\left(\left[\begin{array}{lll}
Q_{i} & U_{i} & V_{i}
\end{array}\right] \cdot\left[\begin{array}{lll}
Q_{j} & U_{j} & V_{j}
\end{array}\right]\right)
$$




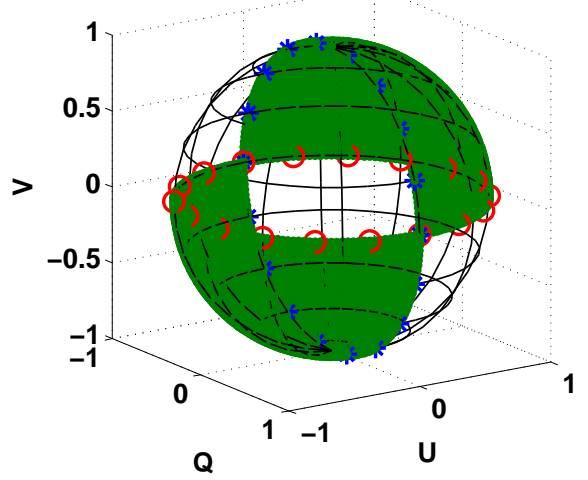

(a)

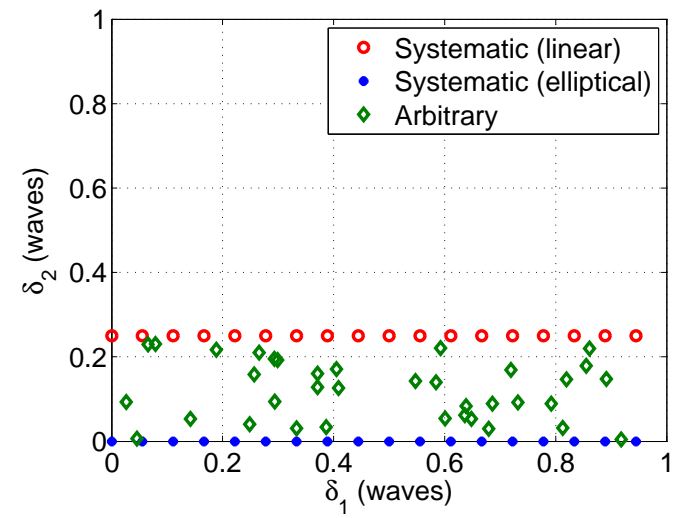

(b)

Figure 3.26: a) Systematic retardance domain shaded in green on the Poincaré sphere. The red circles and blue stars denote the systematic training set. b) Retardance values for the systematic and arbitrary training sets.

The arbitrary training set calibration simulations were performed using the same procedure as that used for exploring misalignment effects in the previous section. In order to compare the results of the arbitrary set with the systematic set, the simulations used the same training set retardance domains. In other words, the arbitrary training set retardance domain matched that of the systematic set, $\delta_{1} \in$ $[0,2 \pi)$ and $\delta_{2} \in[0, \pi / 2)$. The retardance domain is illustrated in Fig. 3.26a, which shows the Poincaré sphere with the domain shaded in green. Additionally, as a point of reference, the figure also shows the systematic training set in red circles and blue stars. Figure 3.26b shows the retardance values for the systematic training set and a given arbitrary training set. From equation (3.27), the average pair-wise distance between the systematic Stokes vectors is $92.57^{\circ}$, while that of the arbitrary set is 91.06 ${ }^{\circ}$ In general, the average distance for an arbitrary set will approach, but never equal, that for the sysematic set. 


\section{Training set size}

Another aspect of performance analysis is determining the proper training set size. Previous researchers have used a training set that contains 36 Stokes vectors, although no reason was given for this number [93]. Exploring the training set size consists of using the misalignment simulation at the nominal retardance values for determining the training set size that adequately suppresses alignment effects. Regardless of size, the training set must span the Poincaré sphere [93].

\section{Results and Discussion}

Figures 3.27-3.30 show the results for the four different simulation configurations for minimizing alignment effects. Figure 3.27 shows the Mueller matrix uncertainty at two wavelengths for the Stokes generator misalignments for the low/low retardance values (i.e., 0 waves and 0.25 waves). The next figure, Fig. 3.28, shows the same thing but for high/high retardance values (i.e., 1 wave and 0.75 waves). Figures 3.29 and 3.30 show results from the low/high and high/low simulation configurations, respectively.

As shown in Figs. 3.27-3.30, the nominal retardance values (i.e., low/low) are the most reliable values to use for minimizing misalignment effects. There does not appear to be any benefit to using high retardance values in the polarimeter (Fig. 3.29), which is expected as the calibration technique assumes error exists only in the Stokes 
generator. The calibration technique compensates for small, constant inaccuracies in the polarimeter LCVRs, and assumes any systematic error occurs in the Stokes generator LCVRs. Additionally, Figs. 3.28 and 3.30 show that using high retardance values in the Stokes generator could decrease error in the lower left Mueller matrix elements of $(3,1),(3,2)$, and $(4,1)$, but this comes at the expense of significantly more error in elements $(2,3)$ and $(4,3)$. 

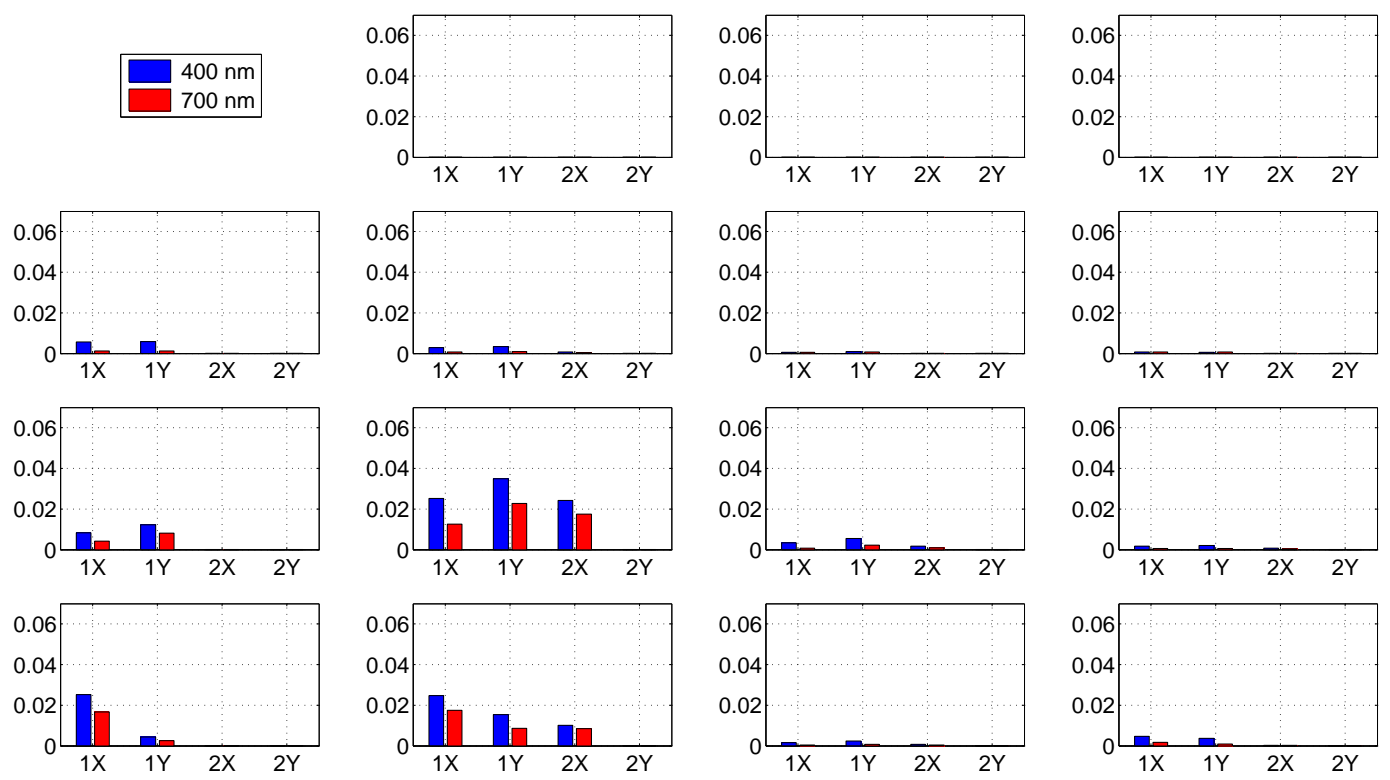

Figure 3.27: Mueller matrix uncertainty at $400 \mathrm{~nm}$ and $700 \mathrm{~nm}$ for low retardance values (i.e., the nominal values) for the Stokes generator and polarimeter for the Stokes generator misalignments.
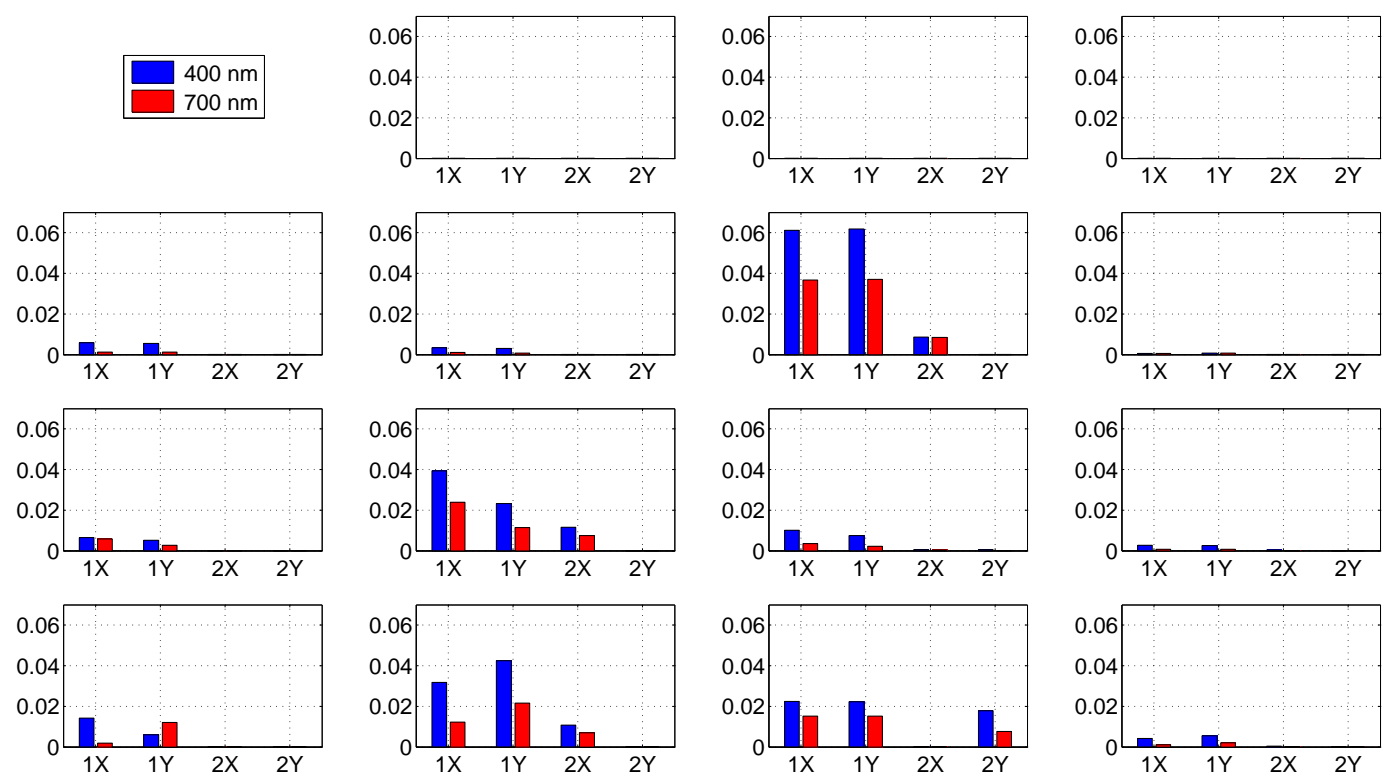

Figure 3.28: Mueller matrix uncertainty at $400 \mathrm{~nm}$ and $700 \mathrm{~nm}$ for the high retardance values for the Stokes generator and polarimeter for the Stokes generator misalignments. 

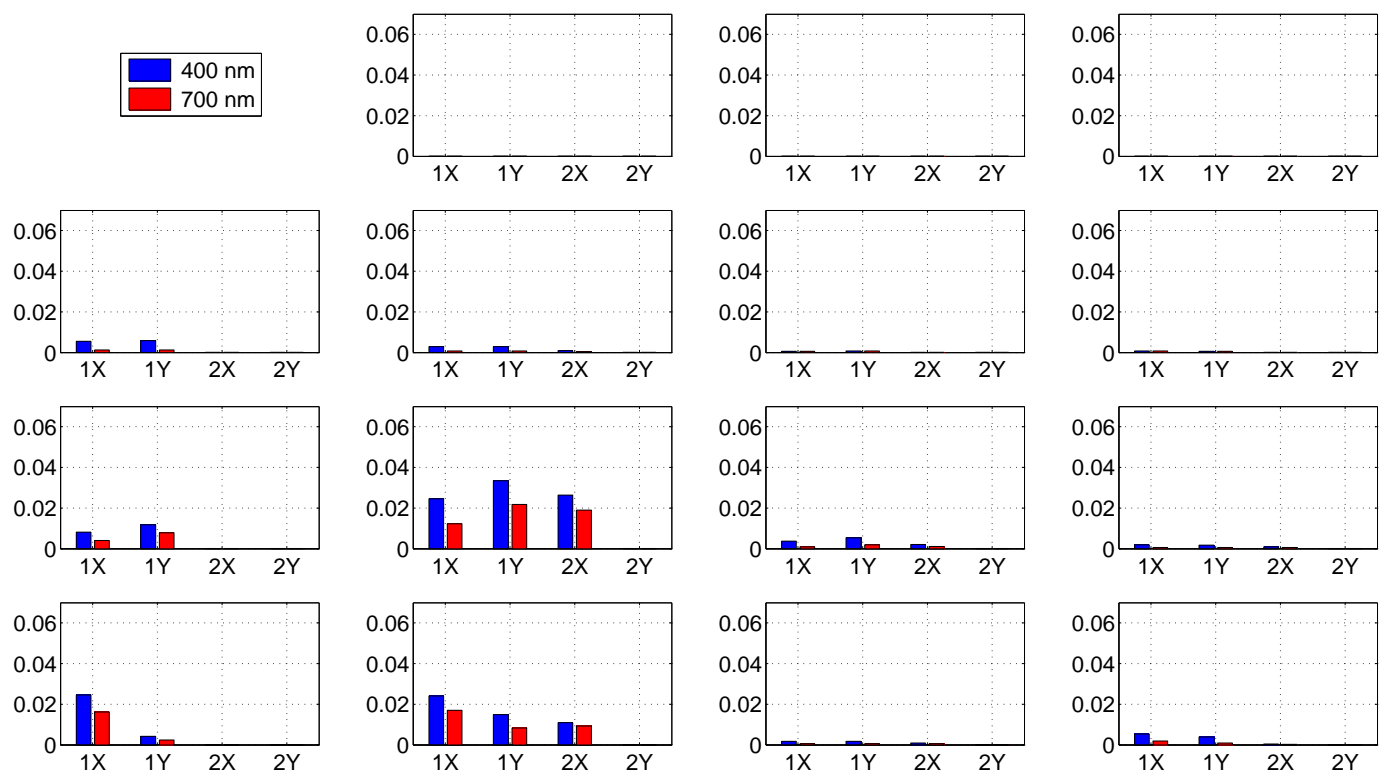

Figure 3.29: Mueller matrix uncertainty at $400 \mathrm{~nm}$ and $700 \mathrm{~nm}$ for low retardance values in the Stokes generator and high retardance values in the polarimeter for the Stokes generator misalignments.
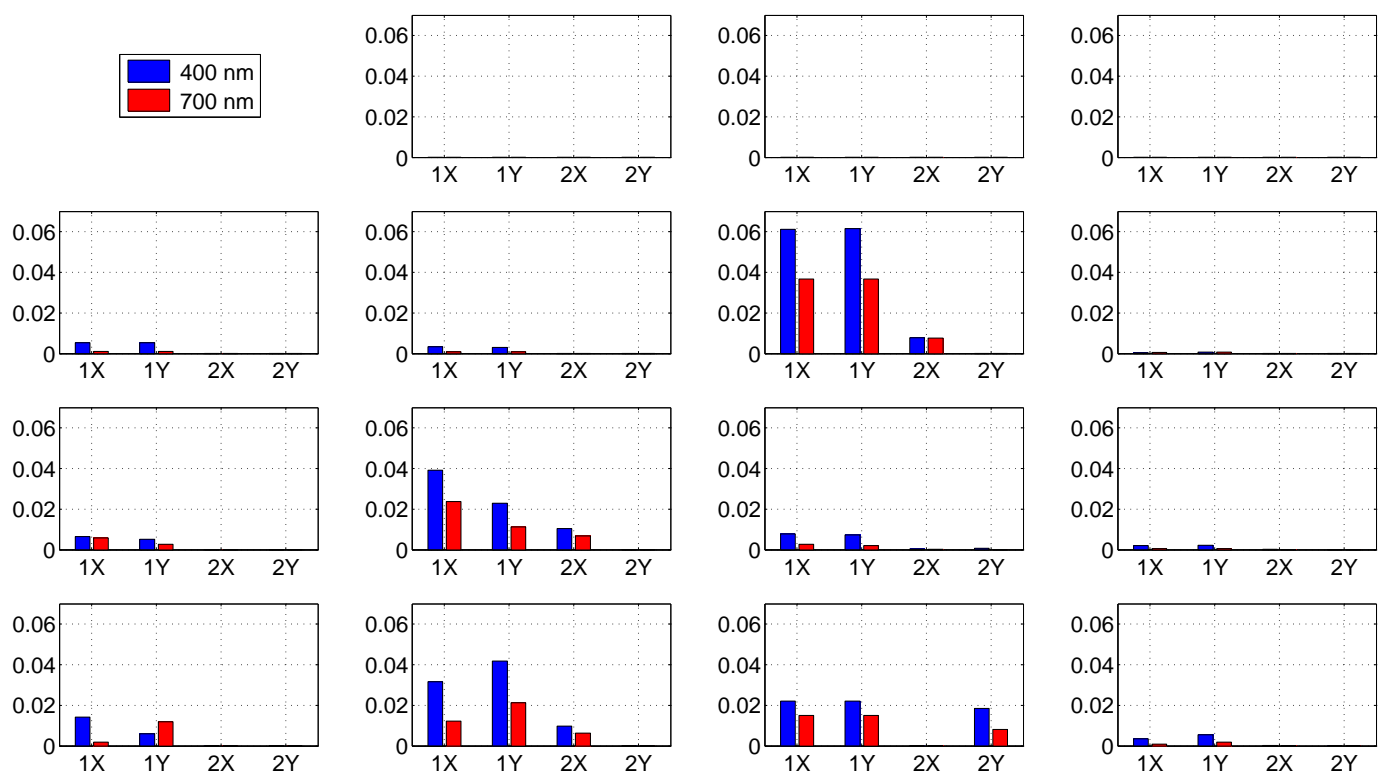

Figure 3.30: Mueller matrix uncertainty at $400 \mathrm{~nm}$ and $700 \mathrm{~nm}$ for high retardance values in the Stokes generator and low retardance values in the polarimeter for the Stokes generator misalignments. 
Figures 3.31-3.34 show the results for the four different simulation configurations for minimizing temperature effects. These figures show data in the same manner as the previous four figures. Figure 3.31 corresponds to the nomial (low/low) retardance values, Fig. 3.32 corresponds to the high/high retardance values, and Figs. 3.33 and 3.34 the low/high and high/low Stokes generator/polarimeter retardance values, respectively.

As shown by Figs. 3.31-3.34, all four sets of retarance values produce error in Mueller matrix elements $(1,2),(1,4),(2,4),(3,4),(4,1),(4,2)$, and $(4,3)$. In addition to this error, the nominal values (i.e., low/low values) produce error in elements $(2,3)$ and $(3,2)$. In contrast, the low/high values produce error only in one of the two elements, $(2,3)$, while the high/low values also produce error in only one of the elements, $(3,2)$. On the other hand, the high/high values produce no error in elements $(2,3)$ and $(3,2)$, but at the expense of slightly more error in the other six elements already exhibiting error. Thus, if it is necessary to have elements $(2,3)$ and $(3,2)$ without error and slightly more error can be tolerated in other Mueller matrix elements, then using the high/high retardance values can be beneficial.

If misalignment is present, using high retardance values for both the Stokes generator and polarimeter produces significant error in the $(2,3)$ and $(3,2)$ elements (see Fig. 3.28). Consequently, using high retardance values is only beneficial when one is confident of proper alignment of the Stokes generator. 

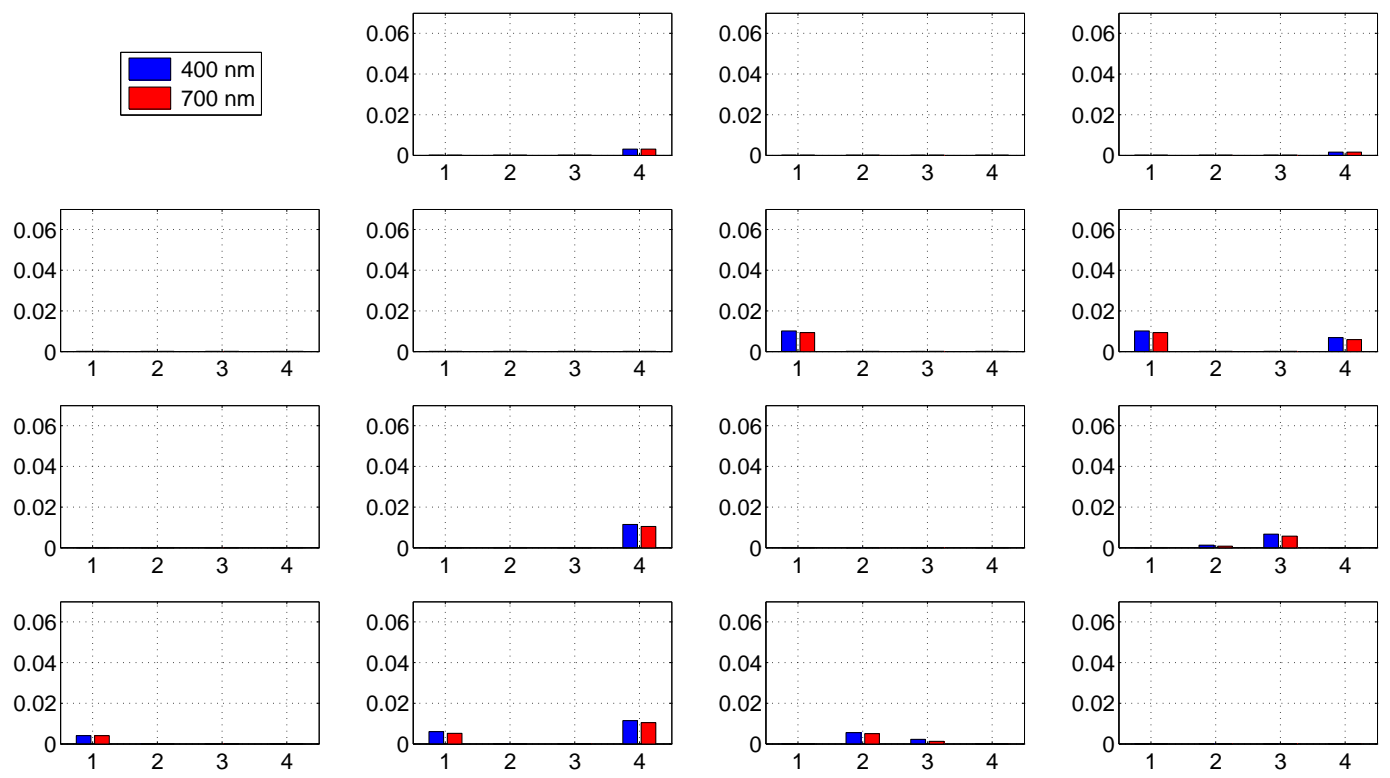

Figure 3.31: Mueller matrix uncertainty at $400 \mathrm{~nm}$ and $700 \mathrm{~nm}$ for low retardance values (i.e., the nominal values) for the Stokes generator and polarimeter for the temperature perturbations.
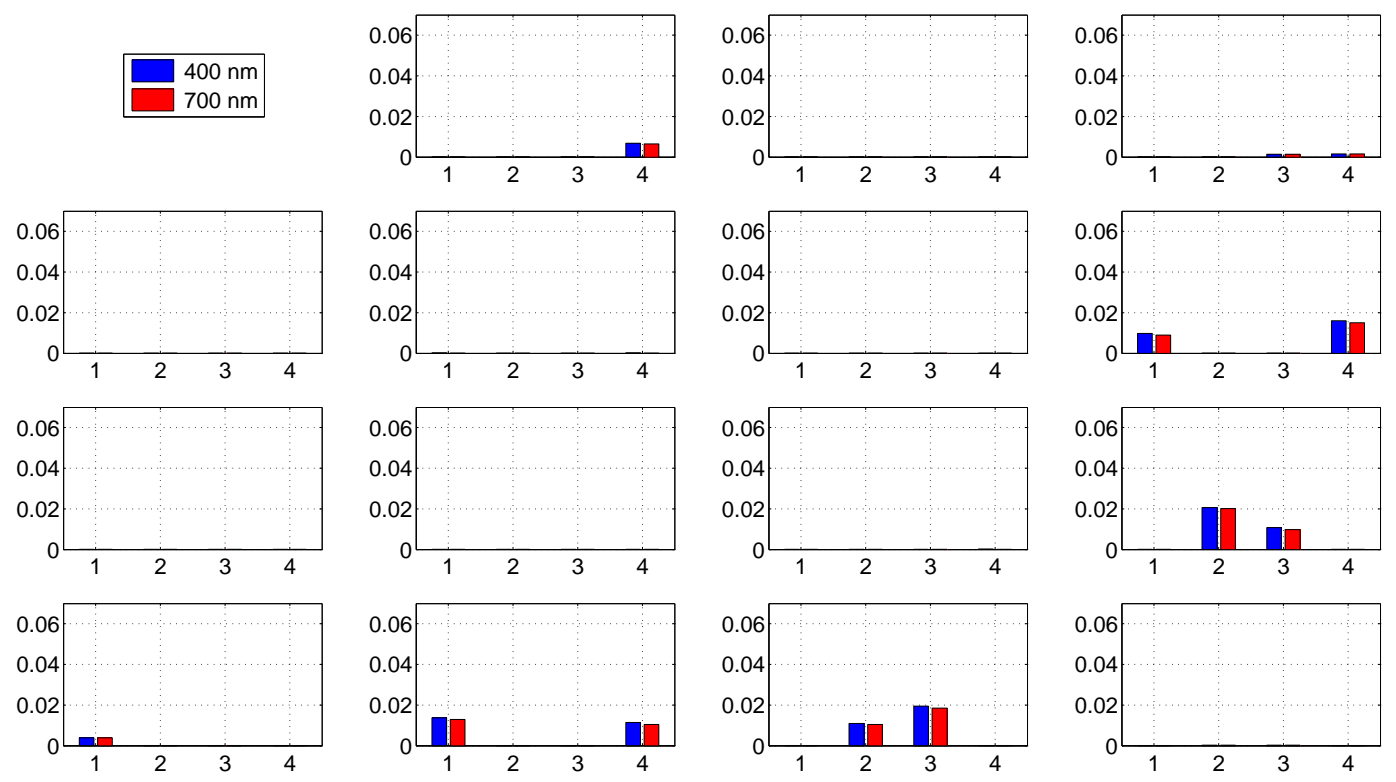

Figure 3.32: Mueller matrix uncertainty at $400 \mathrm{~nm}$ and $700 \mathrm{~nm}$ for the high retardance values for the Stokes generator and polarimeter for the temperature perturbations. 

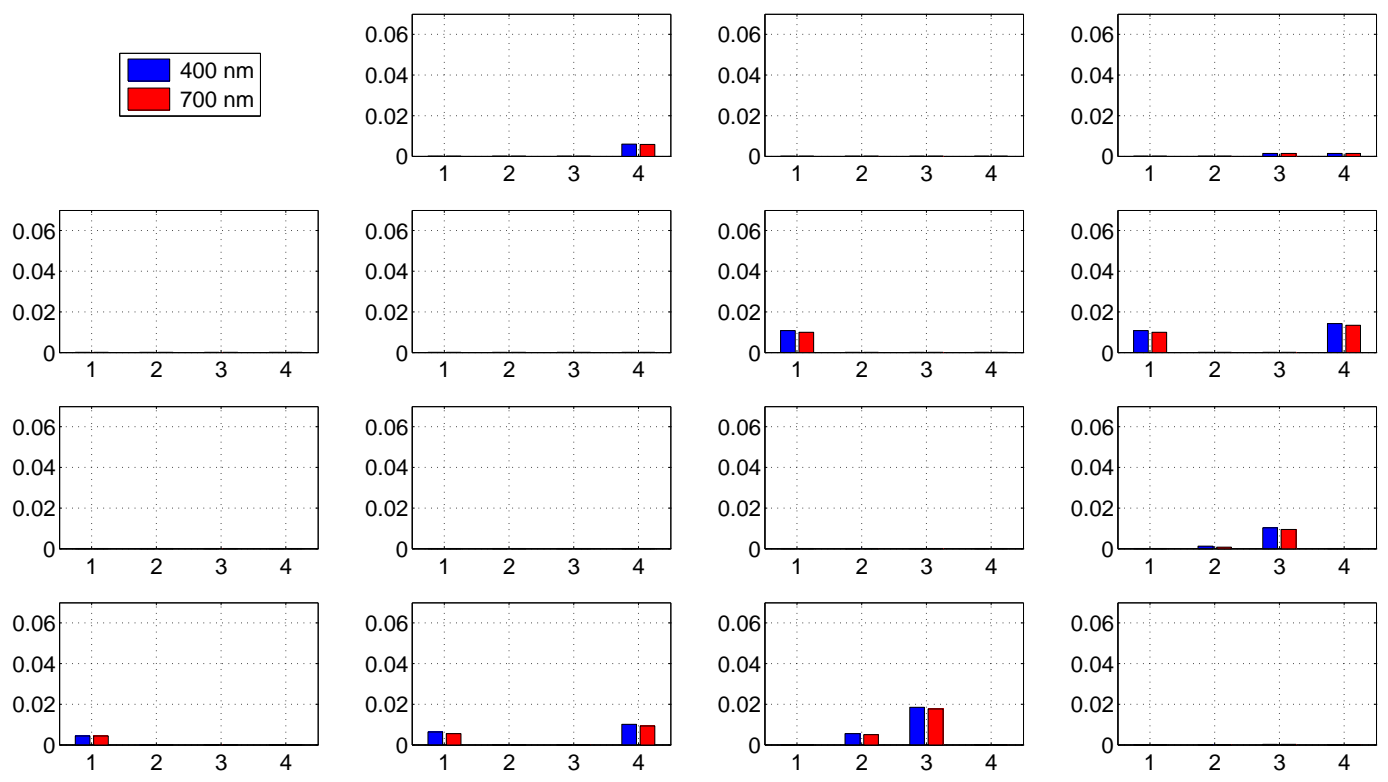

Figure 3.33: Mueller matrix uncertainty at $400 \mathrm{~nm}$ and $700 \mathrm{~nm}$ for low retardance values in the Stokes generator and high retardance values in the polarimeter for the temperature perturbations.
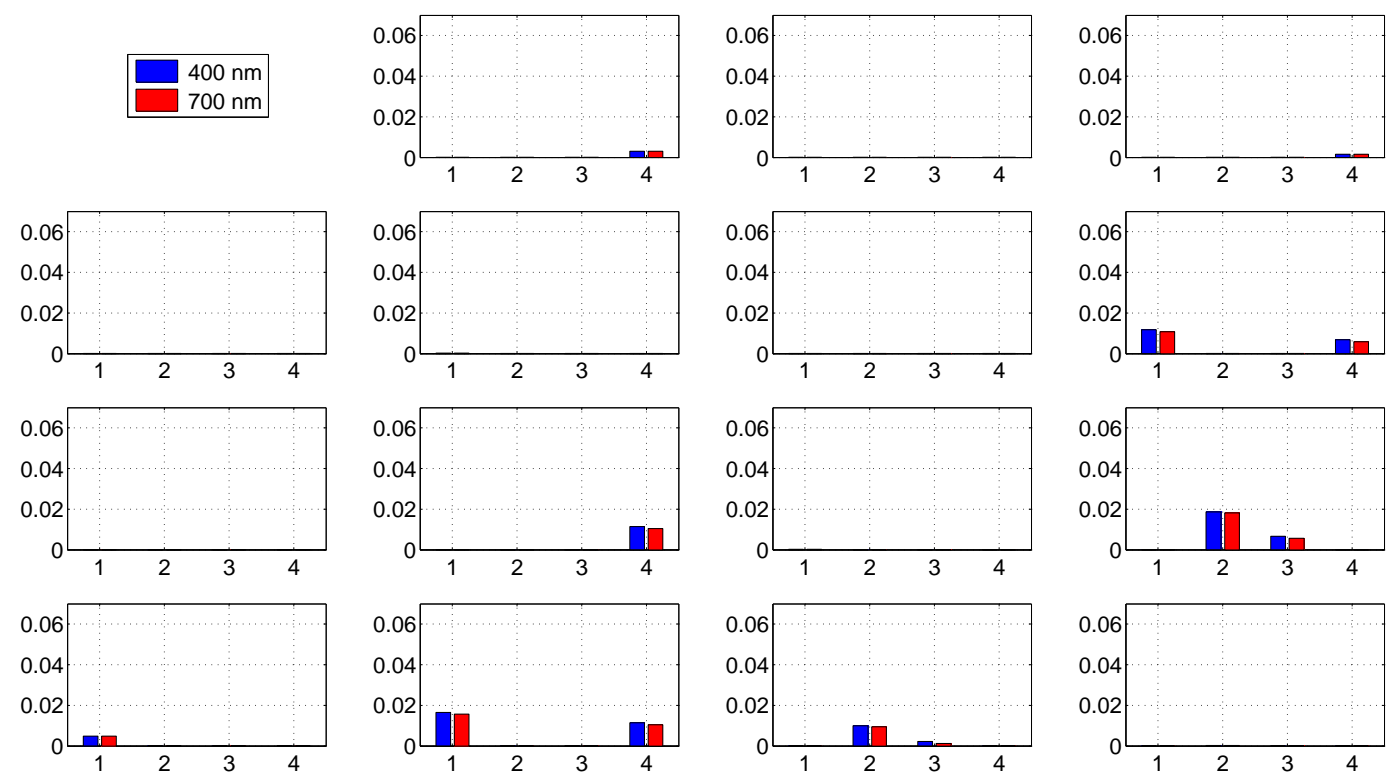

Figure 3.34: Mueller matrix uncertainty at $400 \mathrm{~nm}$ and $700 \mathrm{~nm}$ for high retardance values in the Stokes generator and low retardance values in the polarimeter for the temperature perturbations. 
The next four figures show Mueller matrix uncertainty for the arbitrary Stokes vector training set at $700 \mathrm{~nm}$ for the four sets of retardance values when misalignment is present. Similar error signatures exist for $400 \mathrm{~nm}$, but with larger error magnitudes (data not shown). Figure 3.35 shows results from the low/low retardance values, Fig. 3.36 for high/high values, Fig. 3.37 for low/high values, and Fig. 3.38 for high/low values. Additionally, uncertainty for the corresponding systematic set is shown for comparison. In general, Figs. 3.35-3.38 show that in terms of overall Mueller matrix uncertainty, there is minimal benefit to using an arbitrary training set. However, for specific Mueller matrix elements, there could be advantages, which is discussed next.

Two cases support the use of an arbitrary training set. For the first case, if the user is concerned strictly with elements $(3,1)$ and $(4,2)$, then an arbitrary set may be useful, regardless of the tuning curve region and if there is minimal misalignment for LCVR 2x. The second case would be when the user is concerned strictly with minimizing error in element $(2,3)$. For this case, the user would have to use high retardance values in at least the Stokes generator to remove error from temperature effects. High Stokes generator retardance values, however, introduce error if misalignment is present. Thus, to counteract the alignment effects, an arbitrary training set could be used, as long as LCVR 2x exhibited minimal misalignment. 

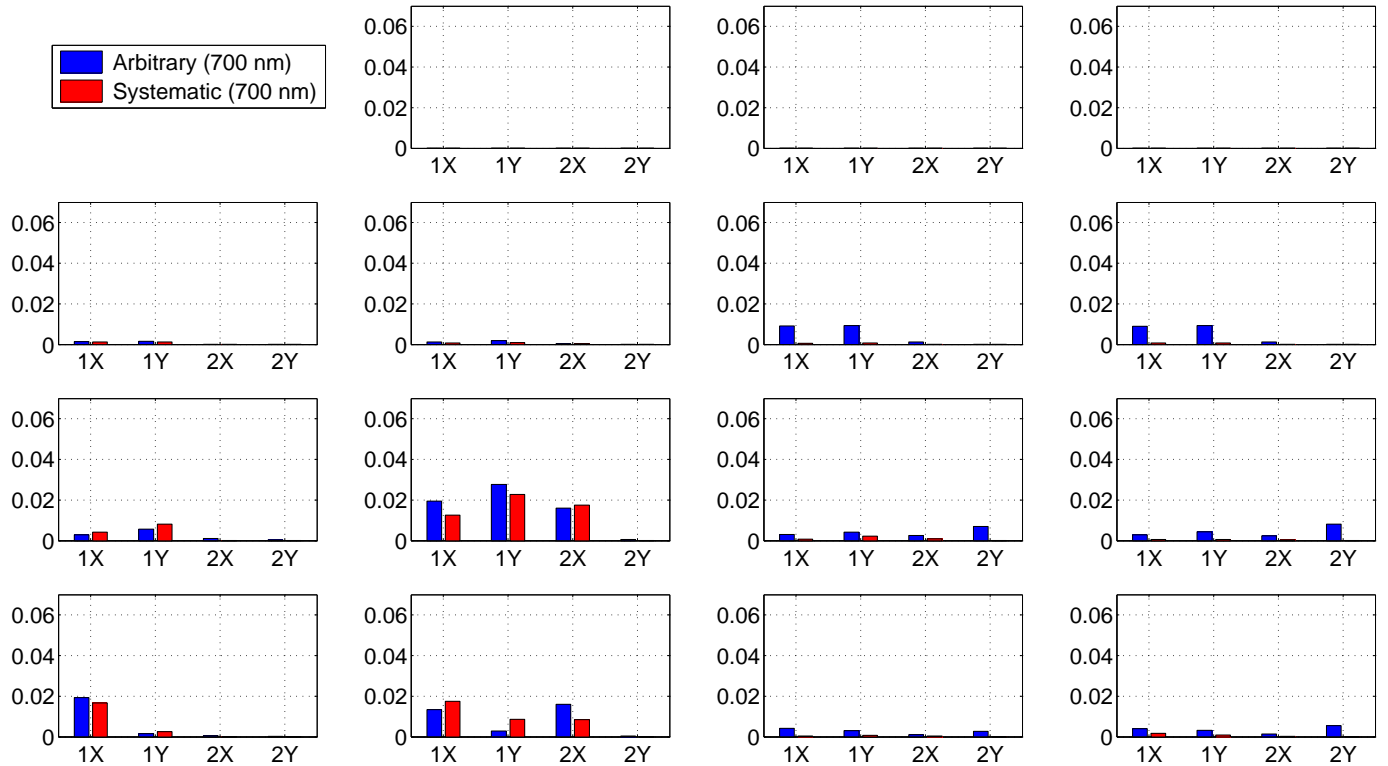

Figure 3.35: Mueller matrix uncertainty at $700 \mathrm{~nm}$ for low retardance values (i.e., the nominal values) for both the Stokes generator and polarimeter for the arbitrary and systematic training sets.
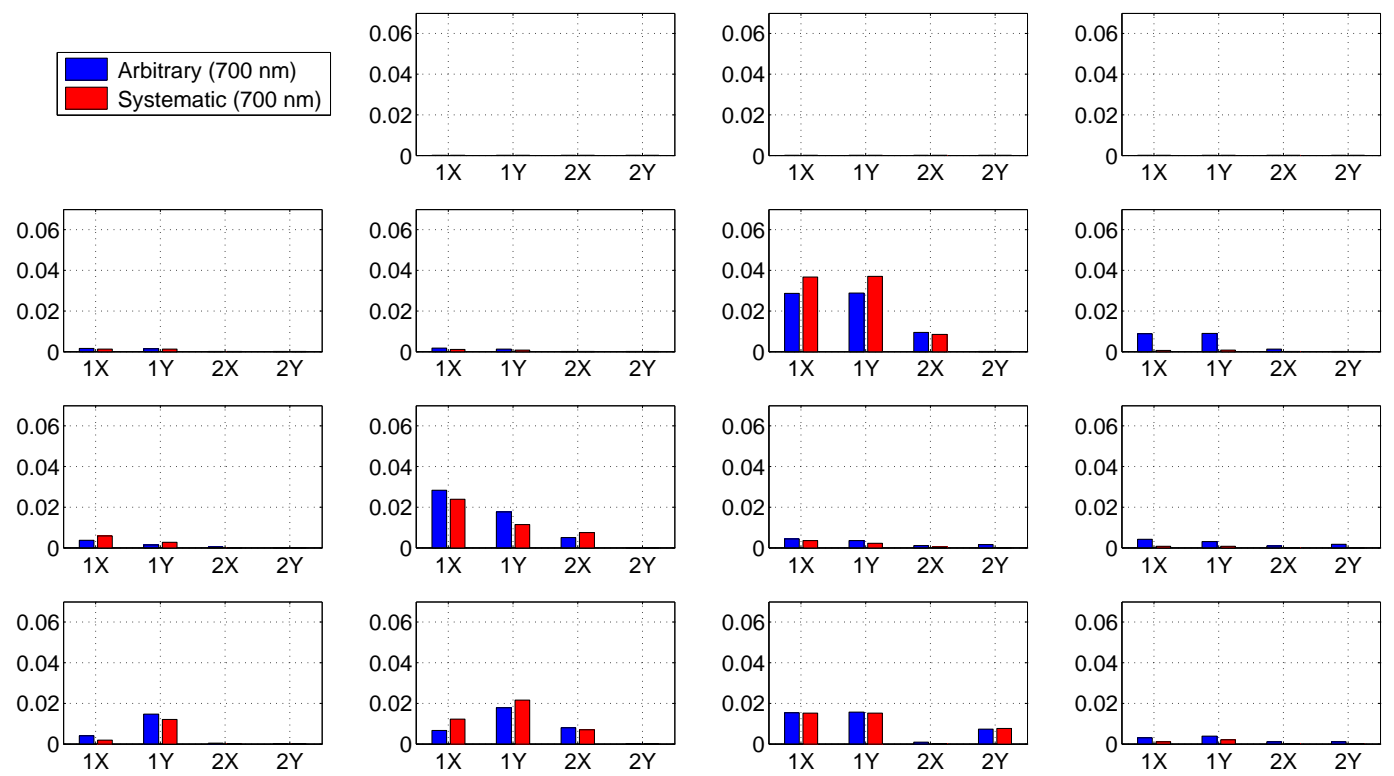

Figure 3.36: Mueller matrix uncertainty at $700 \mathrm{~nm}$ for the high retardance values for both the Stokes generator and polarimeter for the arbitrary and systematic training sets. 

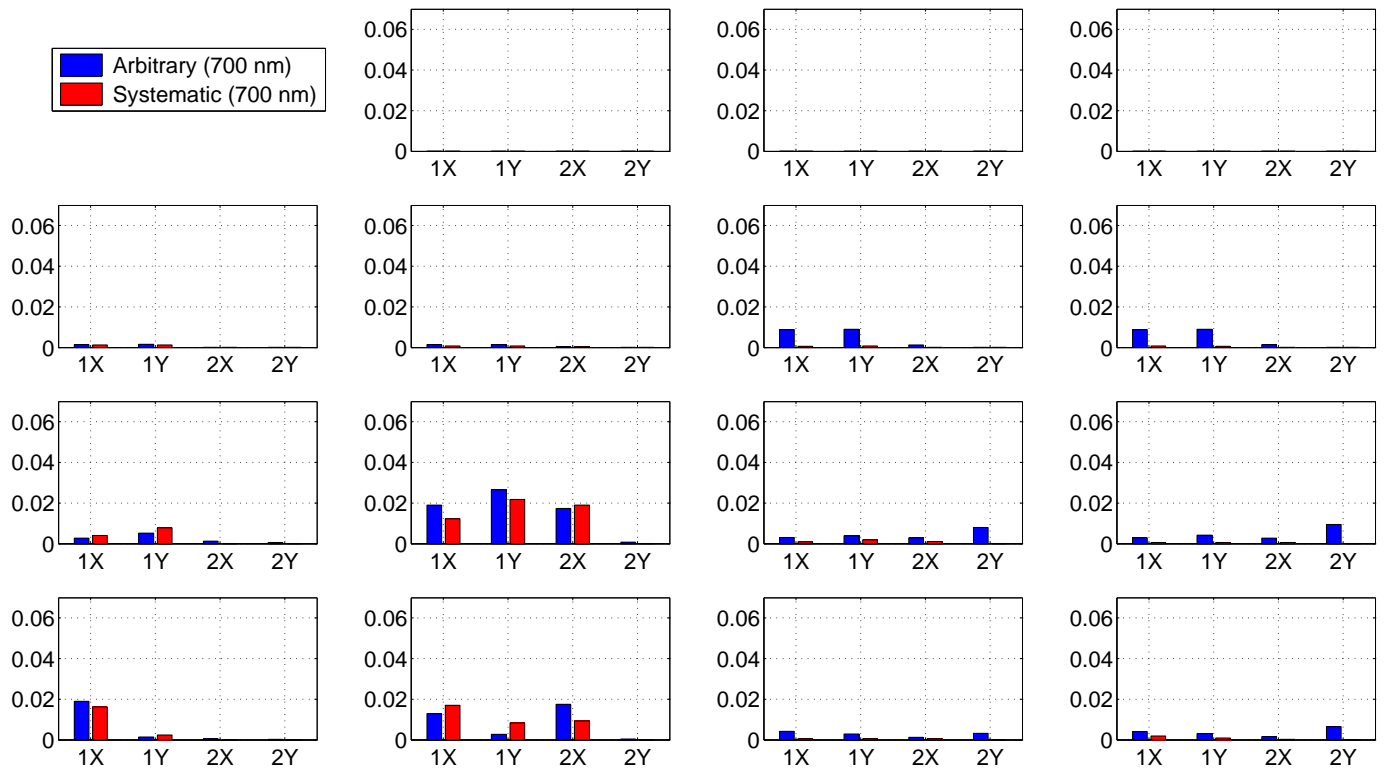

Figure 3.37: Mueller matrix uncertainty at $700 \mathrm{~nm}$ for low retardance values in the Stokes generator and high retardance values in the polarimeter for the arbitrary and systematic training sets.
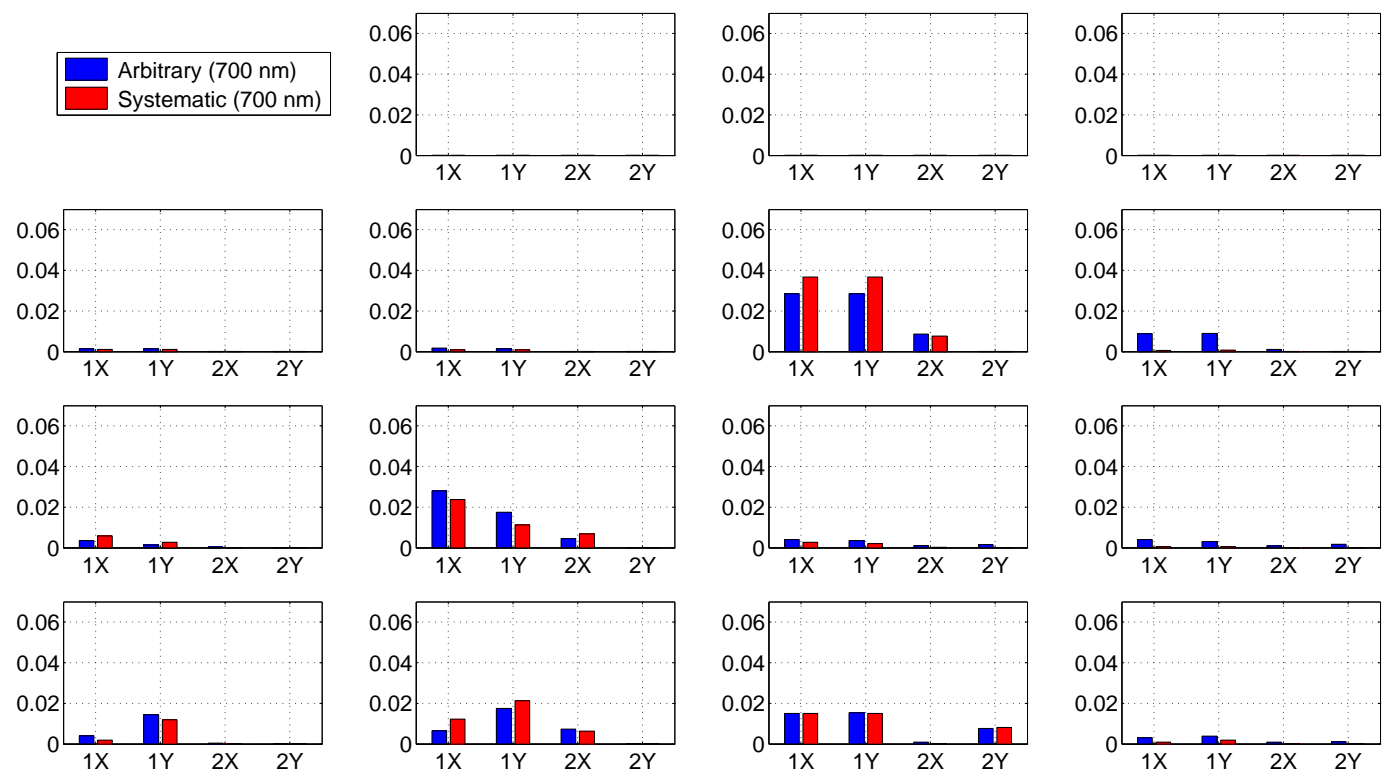

Figure 3.38: Mueller matrix uncertainty at $700 \mathrm{~nm}$ for high retardance values in the Stokes generator and low retardance values in the polarimeter for the arbitrary and systematic training sets. 
Figures 3.39-3.43 show the effect of varying the number of training set Stokes vectors for the nominal (low/low) retardance values when misalignment is present. There is little variation between the results, which suggests that the system performs similarly when calibrated with a 6 -member or 12 -member training set compared to a 48-member set. Employing a 6- or 12-member training set dramatically reduces calibration time, and also makes multiple re-calibrations more feasible. For example, if the ambient temperature fluctuates significantly, then a smaller training set could facilitate multiple re-calibrations without substantial loss of actual measurement time.
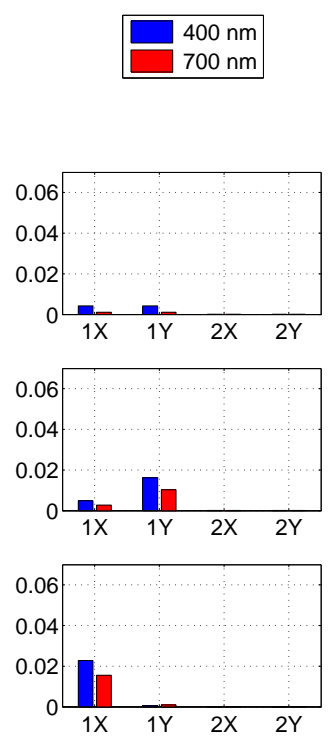
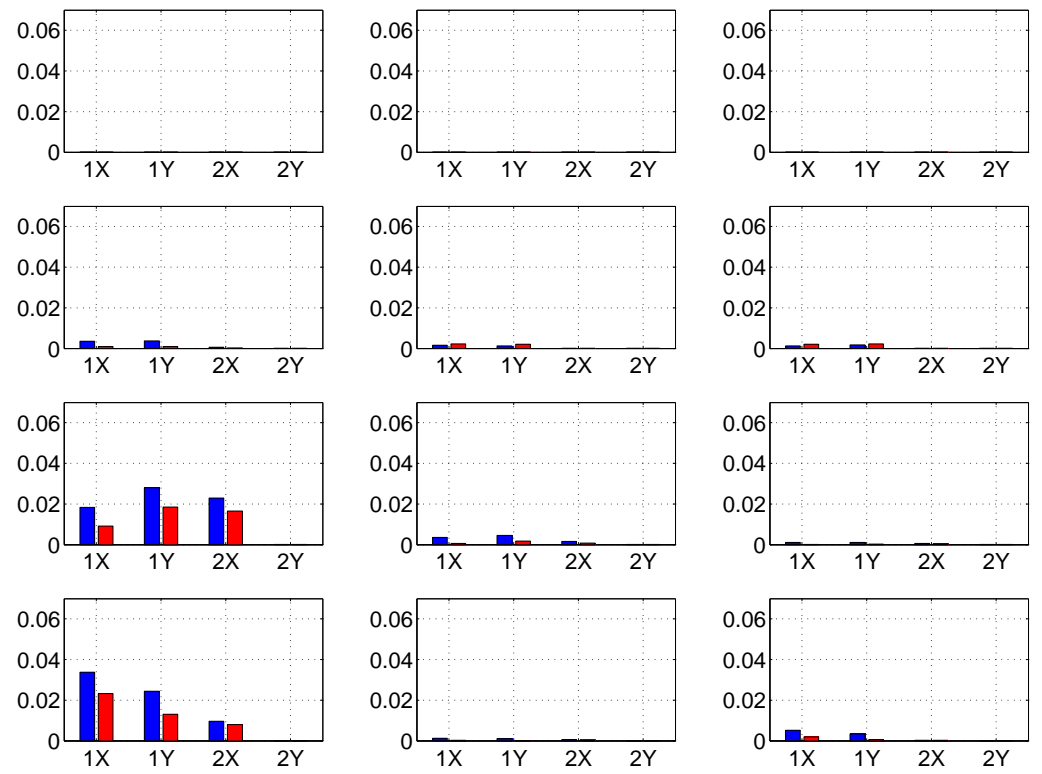

Figure 3.39: Mueller matrix uncertainty at $400 \mathrm{~nm}$ and $700 \mathrm{~nm}$ for low retardance values (i.e., the nominal values) for both the Stokes generator and polarimeter for a 6-member systematic training set. 

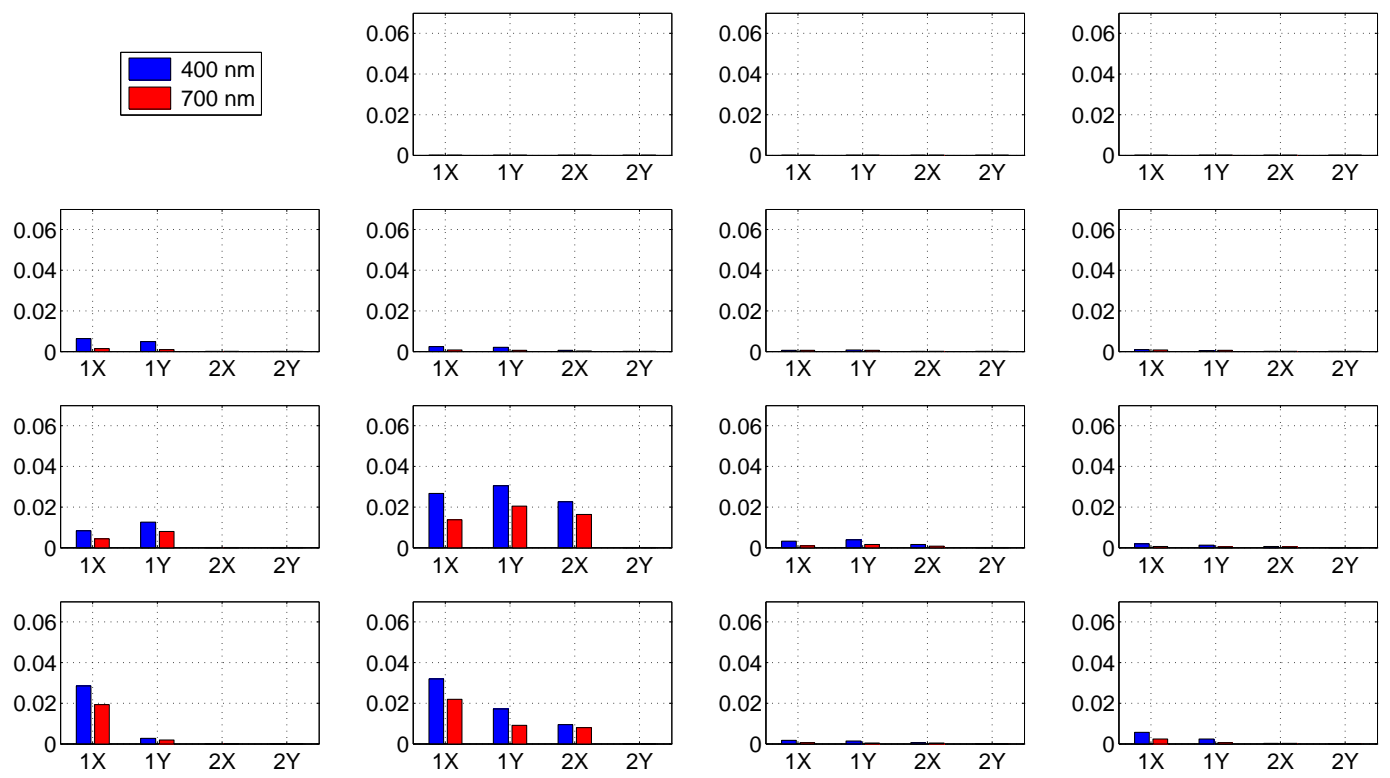

Figure 3.40: Mueller matrix uncertainty at $400 \mathrm{~nm}$ and $700 \mathrm{~nm}$ for low retardance values (i.e., the nominal values) for both the Stokes generator and polarimeter for a 12-member systematic training set.
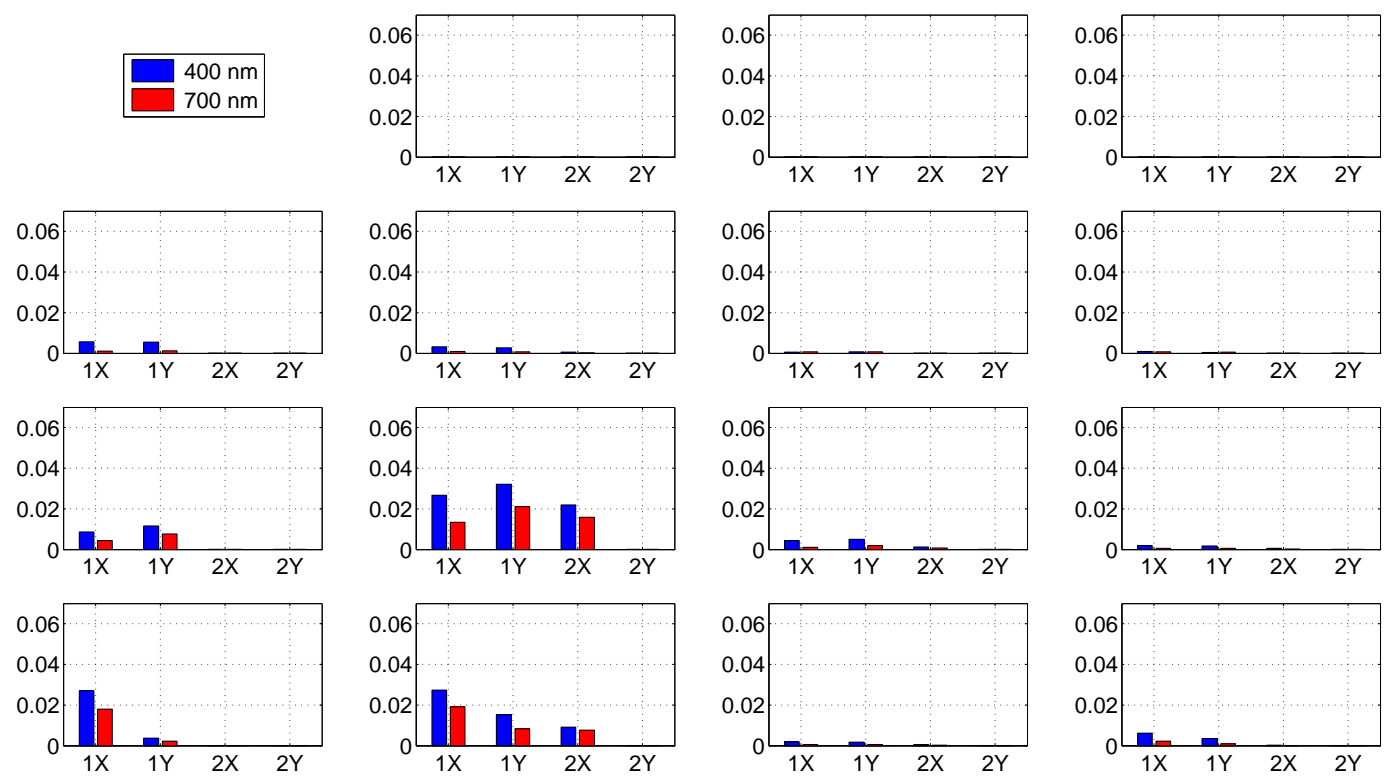

Figure 3.41: Mueller matrix uncertainty at $400 \mathrm{~nm}$ and $700 \mathrm{~nm}$ for low retardance values (i.e., the nominal values) for both the Stokes generator and polarimeter for a 24-member systematic training set. 

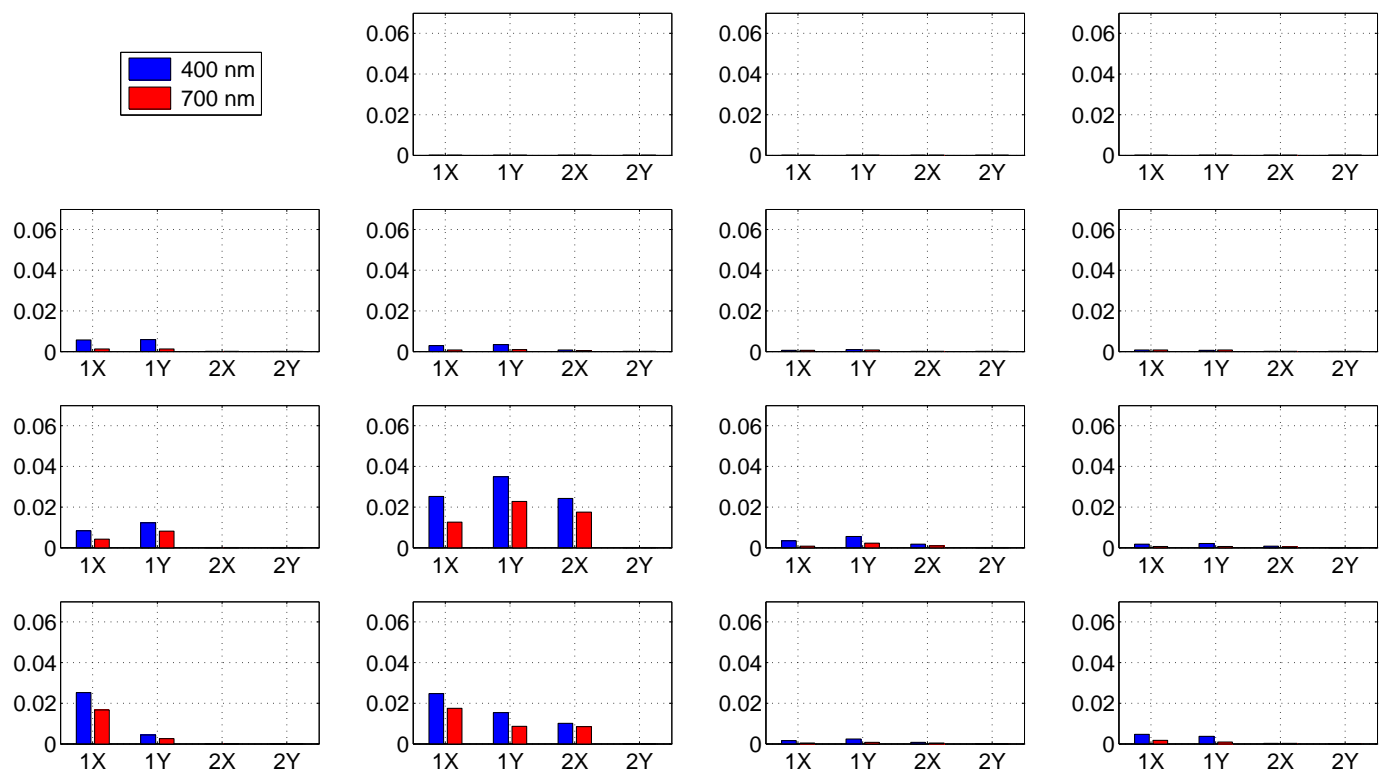

Figure 3.42: Mueller matrix uncertainty at $400 \mathrm{~nm}$ and $700 \mathrm{~nm}$ for low retardance values (i.e., the nominal values) for both the Stokes generator and polarimeter for a 36-member systematic training set.
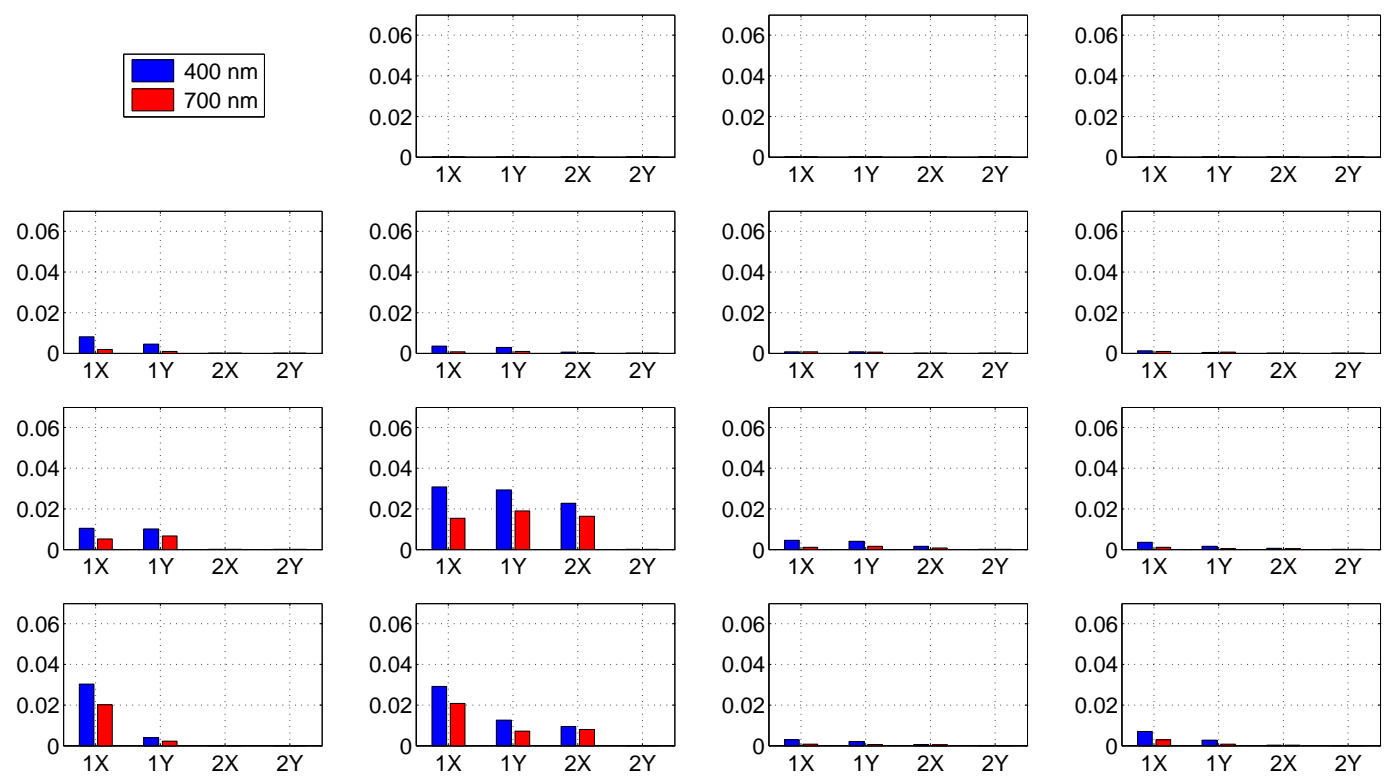

Figure 3.43: Mueller matrix uncertainty at $400 \mathrm{~nm}$ and $700 \mathrm{~nm}$ for low retardance values (i.e., the nominal values) for both the Stokes generator and polarimeter for a 48-member systematic training set. 


\section{Conclusions}

The simulation results presented here provide a roadmap for determining the best methods for suppressing alignment and temperature effects. From these results, it is possible to determine how a particular error source maps to a specific Mueller matrix element (or elements). Additionally, this methodology provides options for minimizing the effect of a particular error source, or for minimizing error in a particular Mueller matrix element.

According to the results, the best method for minimizing alignment error entails using a 6- or 12-member systematic training set with nominal (low/low) retardance values. It is not surprising that the systematic set provides increased error suppression, as it provides maximal Stokes vector spacing. Conversely, a 6- or 12member set unexpectedly provides comparable alignment error mitigation compared to training sets with larger sizes. This is counterintuitive, as a larger calibration set would generally drive error down. This finding is verified in Section 3.4.4, which shows actual calibration measurement results. Additionally, there are two specific cases were it is advantageous to use an arbitrary training set, and these cases only include elements $(3,1),(4,2)$, and $(2,3)$.

The results also show suitable methods for suppressing temperature effects. The results indicate that LCVRs 2 and 3 are the least sensitive to temperature, while LCVRs 1 and 4 are the most sensitive. Because temperature control is only available 
on two of the devices, the LCVRs in positions 1 and 4 have temperature control. Furthermore, there does not appear to be a particular set of retardance settings that provides significantly better error minimization in terms of temperature effects. However, using high retardance values for minimizing temperature effects introduces additional error if alignment effects are present.

The current section addressed a particular aspect of system assessment, performance analysis, through a series of simulations. The final aspect of system assessment, test measurements, is the topic of the next section.

\subsubsection{Test Measurements}

This section discusses results from a variety of test measurements, which are designed to quantify polarimeter accuracy and sensitivity. Results are given for the calibration method described in Section 3.4.1, the performance analysis techniques from Section 3.4.3, and the incidence angle correction factor from Appendix H. In addition, measurements were made on samples with known properties. Calibration samples used for polarimeter assessment include transmission test targets, optical components (linear polarizer, quarter-wave plate, and half-wave plate), and glucose solutions. This section begins by addressing the test targets, followed by the calibration and performance analysis results, and finally the remaining calibration samples. 


\section{Transmission test targets}

Test target measurements were made on two standards, where one standard probes distortion effects and the other determines resolution. The distortion target (ThorLabs, R1L3S3P) has a grid array with $10 \times 10$ micrometer grid spacing. Upon imaging the distortion target, if the horizontal and vertical lines are perpendicular, then the system has no distortion. Figure 3.44a shows an image of the distortion target. This image shows that the lines are straight, thus the system has no distortion.

The target for measuring resolution (1951 USAF target, ThorLabs, R3L3S1N) consists of horizontal and vertical lines at various spacings. The target includes ten different groups of line pairs, where each group further breaks down into six elements made of horizontal and vertical line pairs. For each element, the spacing between the lines equals the width of the lines. The image in Fig. 3.44b captures groups six and seven. Element six of group seven, which is outlined in the colored boxes, exhibits the maximum target resolution (228 line pairs per millimeter, $\mathrm{lp} / \mathrm{mm}$ ) in object space.

Figures $3.45 \mathrm{a}$ and $3.45 \mathrm{~b}$ show cropped and zoomed images of the green and yellow boxes from Fig. 3.44b, respectively. Both sets of lines have a resolution of 228 $\mathrm{lp} / \mathrm{mm}$, or 4.386 microns/lp. Thus, the center-to-center distance for a pair of lines is 4.386 microns. According to Figs. $3.45 \mathrm{a}$ and 3.45b, it takes 9 pixels to cover this distance, which results in an image resolution of 0.487 microns per pixel. In other words, each pixel of the CCD camera represents 0.487 microns in object space. The 


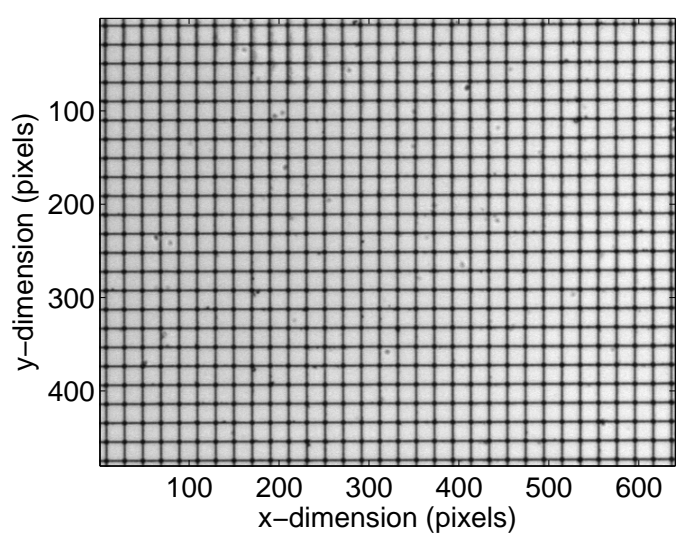

(a)

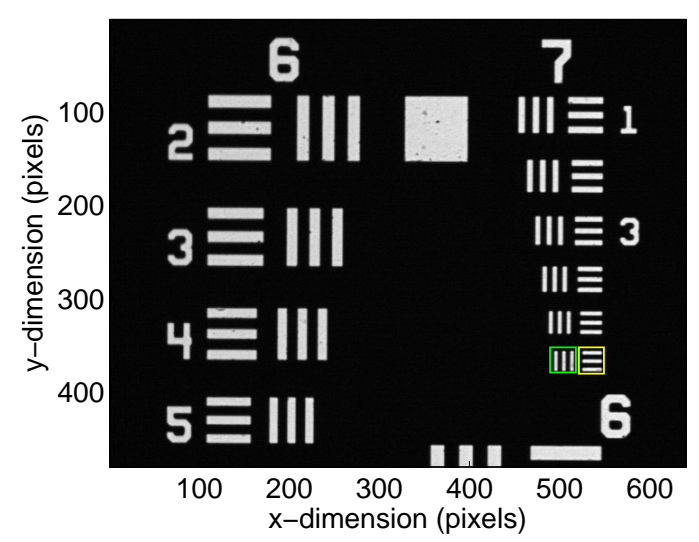

(b)

Figure 3.44: a) Image of the $10 \times 10$ micrometer grid on the distortion target. b) Image of groups six and seven on the resolution target. The colored boxes contain element six of group seven, which has the maximum target resolution of $228 \mathrm{lp} / \mathrm{mm}$ in object space.

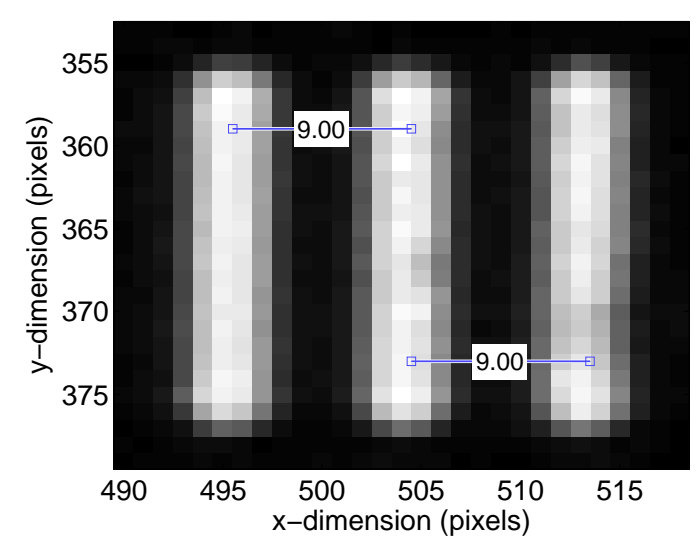

(a)

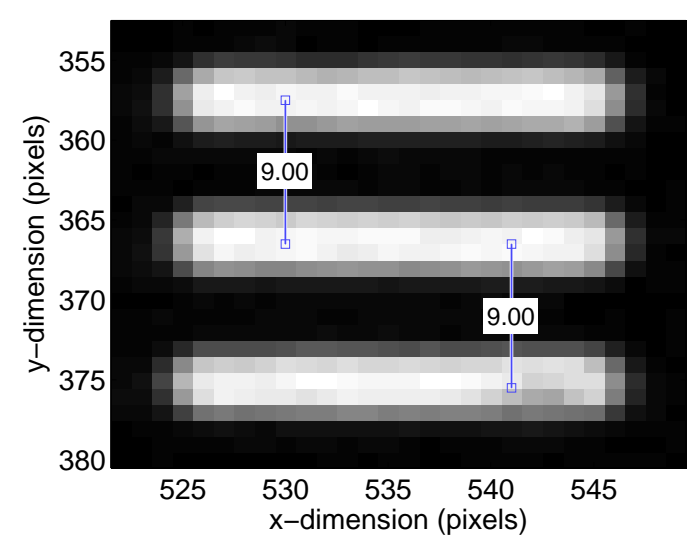

(b)

Figure 3.45: Cropped and zoomed images of the colored boxes from Fig. 3.44b, a) Vertical lines from the green box, b) Horizontal lines from the yellow box. 
physical size of the individual pixels is 9.9 microns. Thus, the actual magnification in the polarimeter is $20.33 \mathrm{x}$. The system was designed to have $20 \mathrm{x}$ magnification. With the resolution now specified, subsequent images will have their $x$ - and $y$-dimensions labeled in microns instead of pixels.

The elements in Fig. 3.44b also are used for estimating the modulation transfer function (MTF), which is the spatial frequency response of an imaging system [120]. The MTF shows the spatial frequency response in terms of contrast, where the contrast is estimated from the minimum and maximum intensity values of a spatial frequency pattern. The contrast $C$ is given by the equation

$$
C=\frac{I_{\max }-I_{\min }}{I_{\max }+I_{\min }}
$$

where $I_{\max }$ and $I_{\min }$ are the the maximum and minimum intensity values, respectively, for a given spatial frequency pattern. Contrast is computed for the 20 spatial frequency patterns in Fig. 3.44b, which consist of ten sets of horizontal lines and ten sets of vertical lines. The MTF is shown in Fig. 3.46 for the both the horizontal and vertical line patterns. In this figure, the maximum spatial frequency in image space is $11.2 \mathrm{lp} / \mathrm{mm}$, which corresponds to $228 \mathrm{lp} / \mathrm{mm}$ divided by the magnification. 


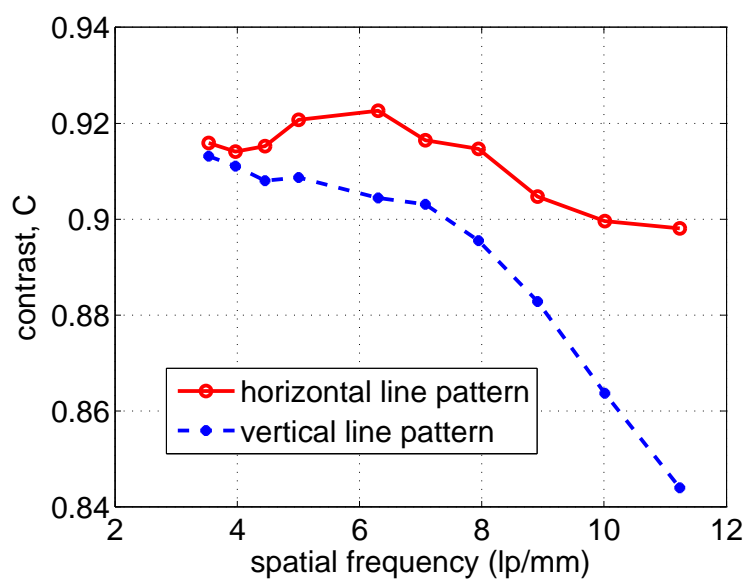

Figure 3.46: Contrast as a function of spatial frequency for the line patterns.

\section{Polarimeter calibration}

Polarimeter calibration establishes a relationship between polarimeter measurements and the incident Stokes vector. The data reduction matrix $\mathbf{W}$ embodies this relationship, and determines the accuracy with which the polarimeter can recover an incident Stokes vector. Computing the condition number of $\mathbf{W}$ provides a metric for assessing the calibration, where a lower value indicates a more accurate measurement system. The theoretical minimum condition number is $\sqrt{3}$, or approximately 1.73 .

Calibration is necessary for each measurement configuration employed, as moving the polarimeter can cause alignment uncertainties. Thus, the system must be calibrated for both the transmission and backscatter configurations. For the transmission configuration, the polarimeter is at an angle of $180^{\circ}$ relative to the Stokes generator. In the backscatter configuration, the polarimeter is rotated around the sample plane such that it makes an angle of $60^{\circ}$ with the Stokes generator (see Fig. 


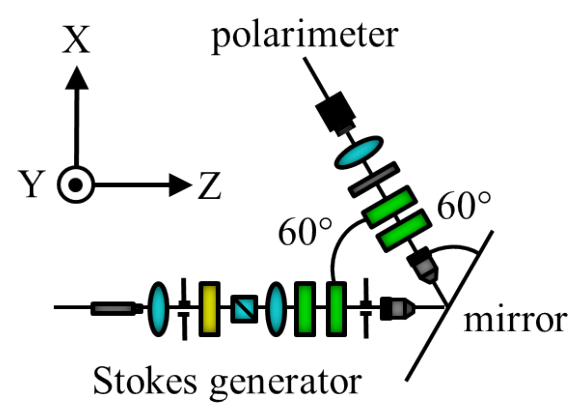

Figure 3.47: Schematic (plan view) of the measurement configuration for measuring specular reflection from the mirror.

3.47). This configuration permits the $30^{\circ}$ specular reflection from the sample to be measured. Calibration is done with a mirror (ThorLabs Protected Silver mirror, PF10-03-P01) in the sample plane. The mirror was rotated in the sample plane such that the polarimeter collected the $30^{\circ}$ specular reflection. Figure 3.47 shows a plan view schematic of the backscatter calibration with the mirror.

Calibration measurements were made in both a transmission and backscatter configuration for a wavelength range of $440-720 \mathrm{~nm}$ at $20 \mathrm{~nm}$ increments. Additionally, subsequent measurements were made for estimating the Mueller matrix of air, which theoretically is the identity matrix. Figure 3.48 shows condition number images at 500 and $720 \mathrm{~nm}$ for a training set consisting of 36 Stokes vectors for both configurations. Figure 3.49 shows the mean and standard deviation of each condition number image as a function of wavelength for the two configurations. Next, Fig. 3.50 shows the mean and standard deviation of each air Mueller matrix image as a function of wavelength for both configurations. Finally, Fig. 3.51 shows the RMS error of the entire air Mueller matrix as a function of wavelength for the two configurations. 

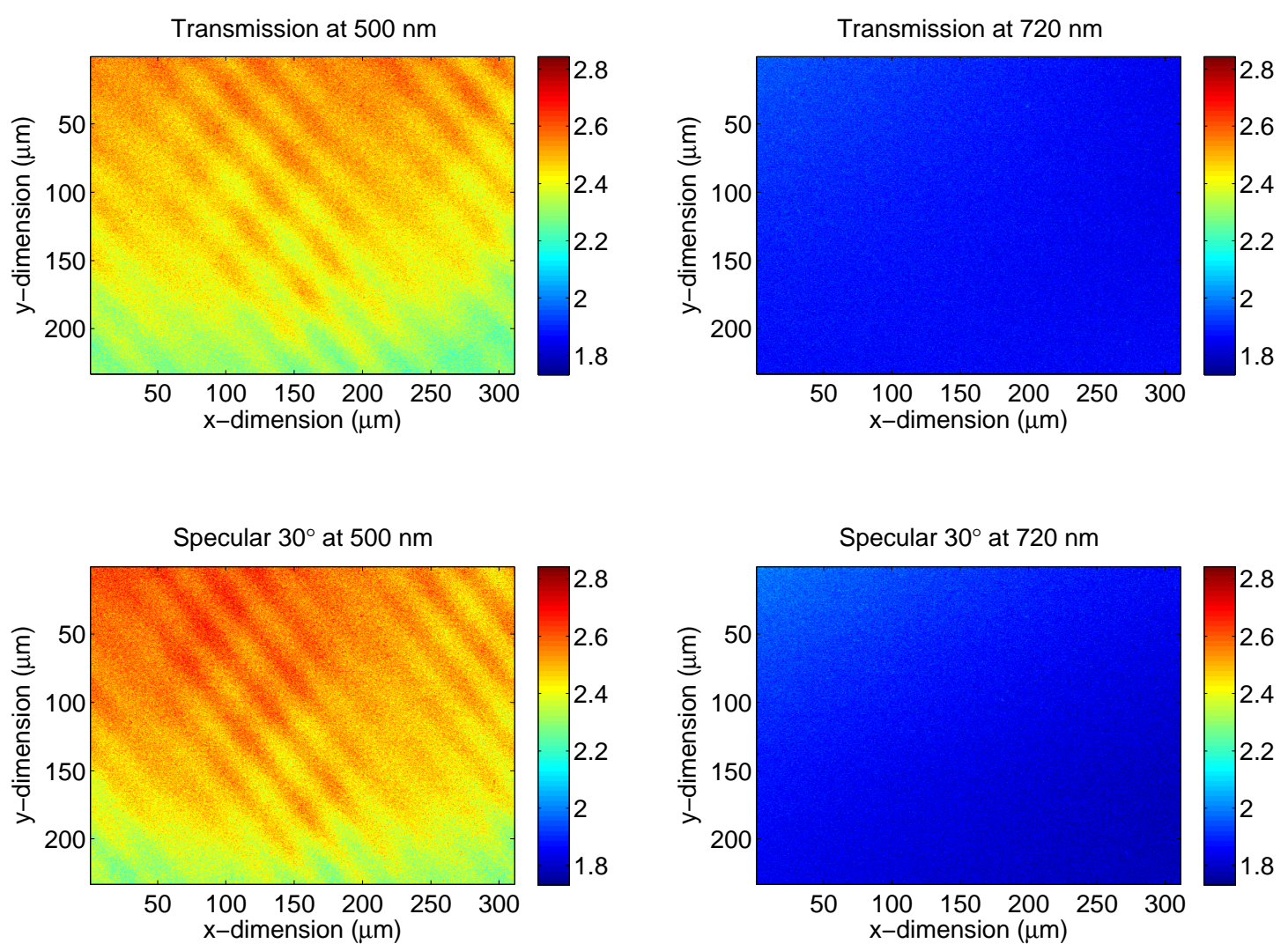

Figure 3.48: Condition number images for the two configurations at two wavelengths.

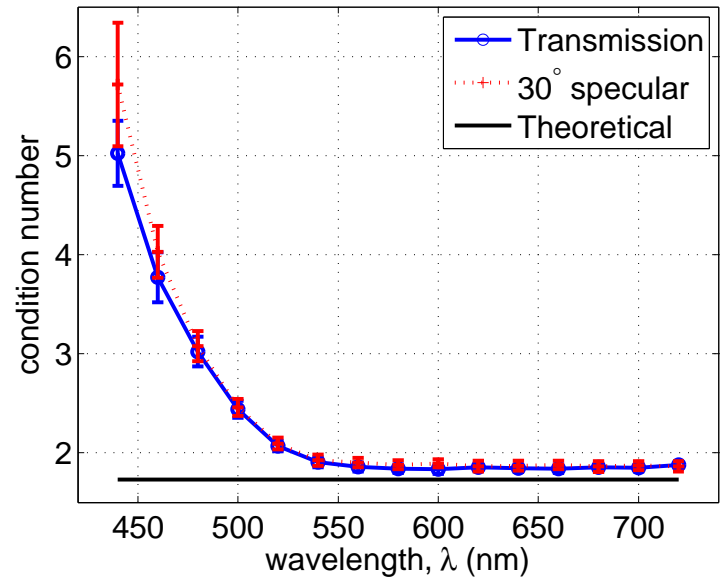

Figure 3.49: Condition number mean and standard deviation as a function of wavelength for the two configurations. 


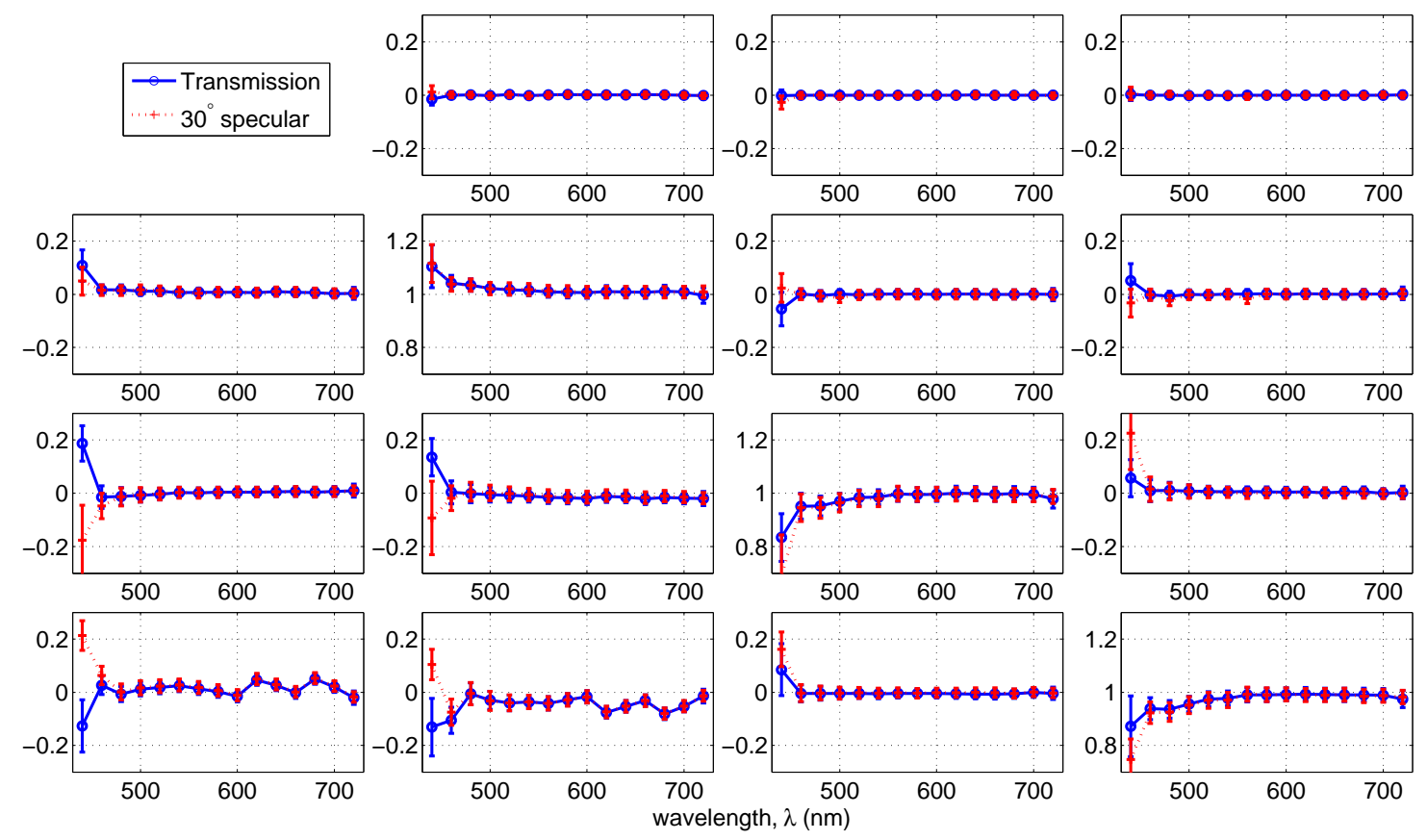

Figure 3.50: Air Mueller matrix mean and standard deviation as a function of wavelength for the two configurations.

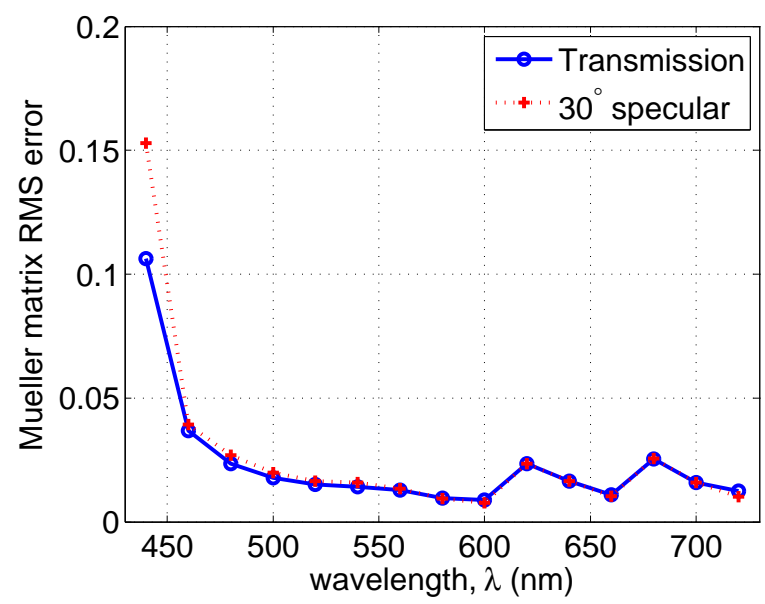

Figure 3.51: RMS error for the entire air Mueller matrix as a function of wavelength for the two configurations. 


\section{Performance analysis measurements (training set size)}

Performance analysis measurements were made for exploring the effects of training set size. A training set with fewer Stokes vectors results in faster calibration times and facilitates more frequent calibrations if there are room temperature changes or if the polarimeter is moved. Figure 3.52 shows the mean and standard deviation of the condition number images as a function of wavelength for training set sizes of 6 , 12, 24, 36, and 48 Stokes vectors. Figure 3.53 shows results from the air Mueller matrix measurements. This figure shows the mean and standard deviation for each Mueller matrix image as a function of wavelength for the five training sets. Finally,

Fig. 3.54 shows the RMS error for the entire Mueller matrix of air as a function of wavelength for the five training set sizes. These figures confirm the simulation results from Section 3.4.3, and that the system performs similarly when calibrated with a 6or 12-member training set compared to larger training sets. In terms of the results from Fig. 3.54, training sets with fewer members exhibit an RMS error for the entire Mueller matrix that is $\approx 0.5 \%$ higher than larger training sets at wavelengths longer than $500 \mathrm{~nm}$. 


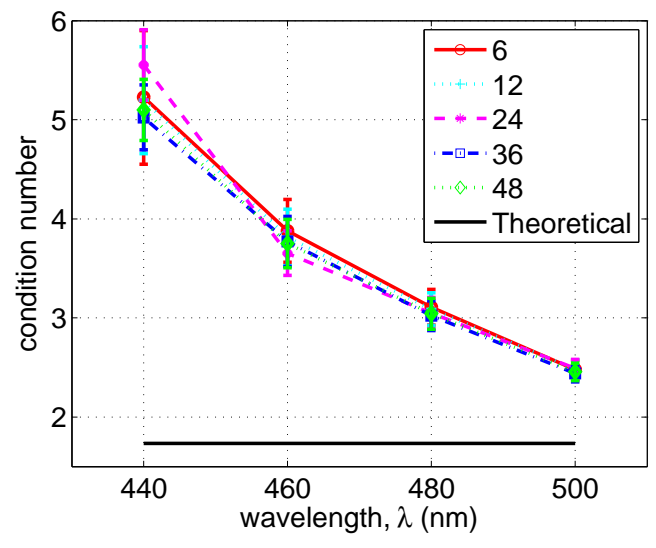

(a) $\lambda=440-500 \mathrm{~nm}$

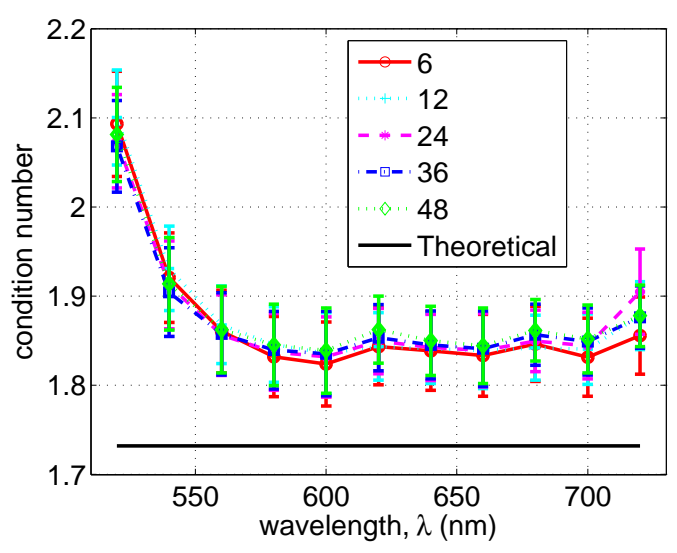

(b) $\lambda=520-720 \mathrm{~nm}$

Figure 3.52: Condition number mean and standard deviation as a function of wavelength for the five training set sizes. The data are separated into two wavelength regions for clearer interpretation.
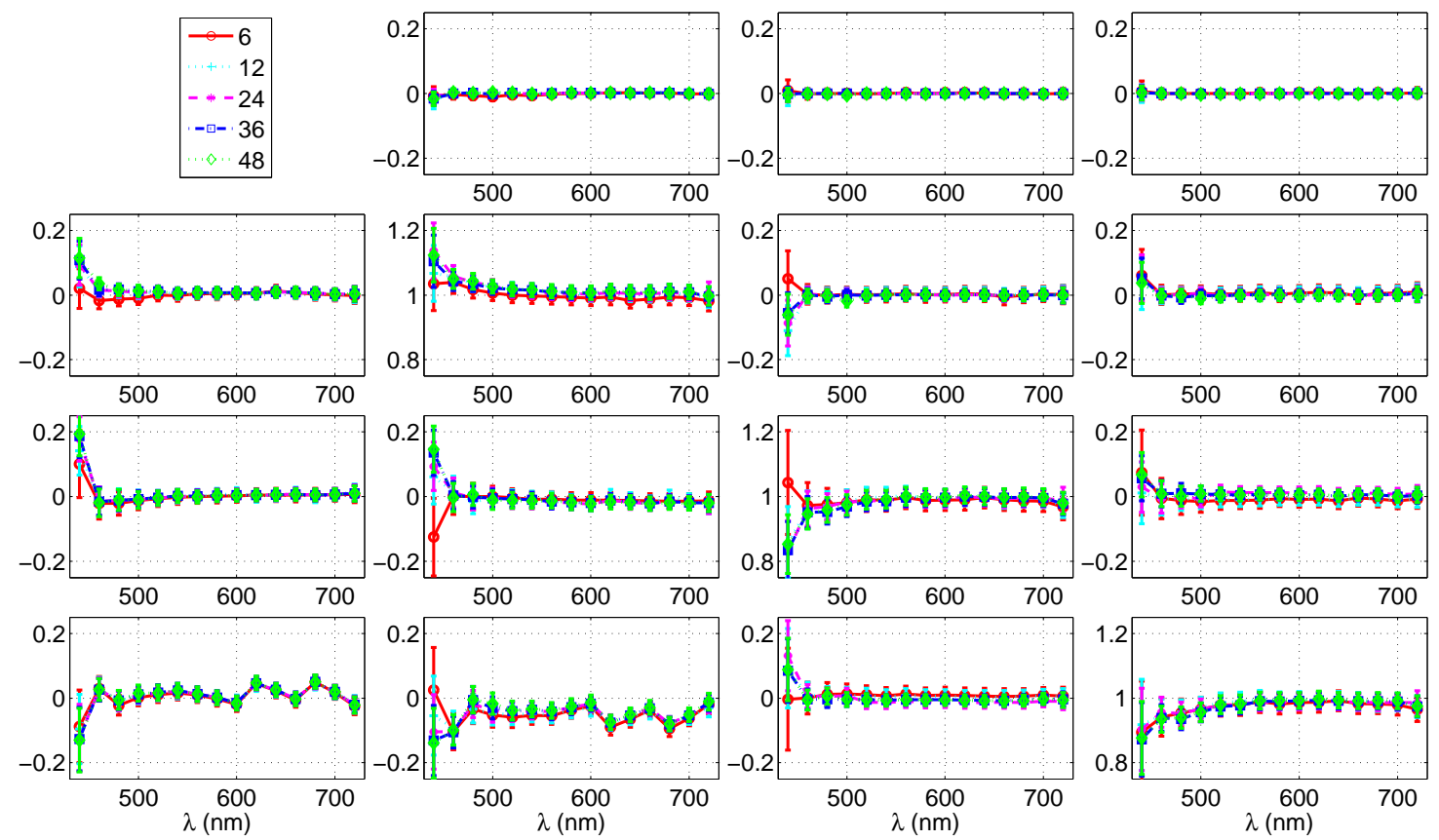

Figure 3.53: Air Mueller matrix mean and standard deviation as a function of wavelength for the five training set sizes. 


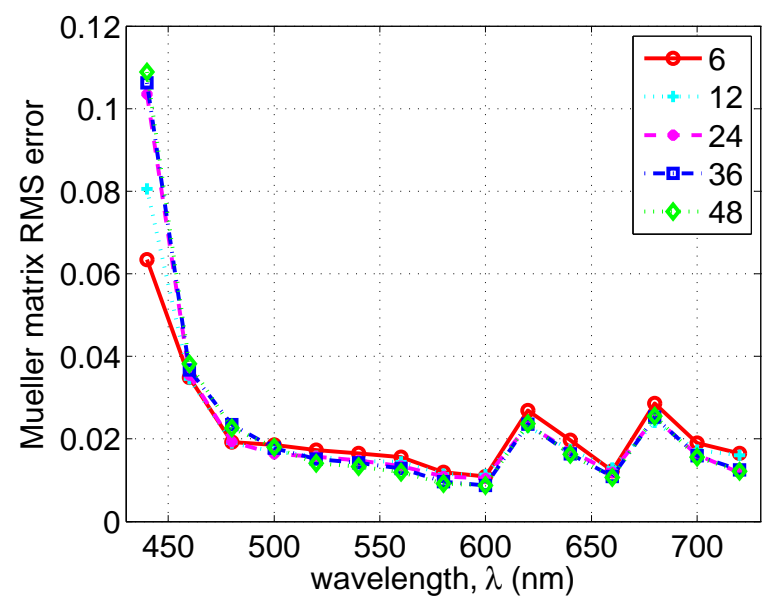

Figure 3.54: RMS error for the entire air Mueller matrix as a function of wavelength for the five training set sizes.

\section{Optical components}

Measuring samples with known properties serves as a method for assessing the accuracy and sensitivity of the polarimeter. Calibration samples used for polarimeter assessment include a linear polarizer, quarter-wave plate, and a half-wave plate. Although a complete Mueller matrix is obtained for each calibration sample, the samples provide an assessment of specific Mueller matrix elements. For example, measurements on the linear polarizer emphasize the upper left $3 \times 3$ submatrix (i.e., Mueller matrix elements that represent linear polarization conversions). The waveplate measurements represent the ability of the polarimeter to probe birefringence (specifically the lower $3 \times 3$ submatrix)

Although the optical components have known properties, there is uncertainty about specific aspects of the components. For both the polarizer and waveplates, 
this includes uncertainty in orientation of the device principal axes. Additionally, for the waveplates, there is uncertainty in the retardance, which is due to incident angle effects and spectral effects. Thus, an uncertainty equation can be used for bounding the error due to orientation and retardance. In general, the uncertainty $\Delta F$ is

$$
\Delta F(x, y)= \pm\left[\left(\Delta x \frac{\partial F(x, y)}{\partial x}\right)^{2}+\left(\Delta y \frac{\partial F(x, y)}{\partial y}\right)^{2}\right]^{\frac{1}{2}}
$$

where $F(x, y)$ is the function of interest, and $x$ and $y$ are parameters to be investigated. For the polarizers and waveplates, $F(x, y)$ corresponds to the functional form of a given Mueller matrix element, $x$ corresponds to component orientation, and $y$ corresponds to retardance.

\section{Polarizer}

The linear polarizer (Thorlabs, 10LP-VIS-B) was oriented at an angle of $\theta \approx 0^{\circ}$, such that it transmitted horizontally polarized light. The Mueller matrix for a horizontal linear polarizer consists of 1 's in the upper left $2 \times 2$ submatrix, and 0 's everywhere else (see Appendix B for the functional form of the Mueller matrix elements). There are no retardance effects associated with the polarizer, so equation (3.29) employed only $\theta$. For example, for the $(1,2)$ Mueller matrix element, the analytic model is $F(\theta)=(1 / 2) \cos 2 \theta$ and $\partial F / \partial \theta=-\sin 2 \theta$. Figure 3.55 shows the mean and standard

deviation of each polarizer Mueller matrix image as a function of wavelength, along 


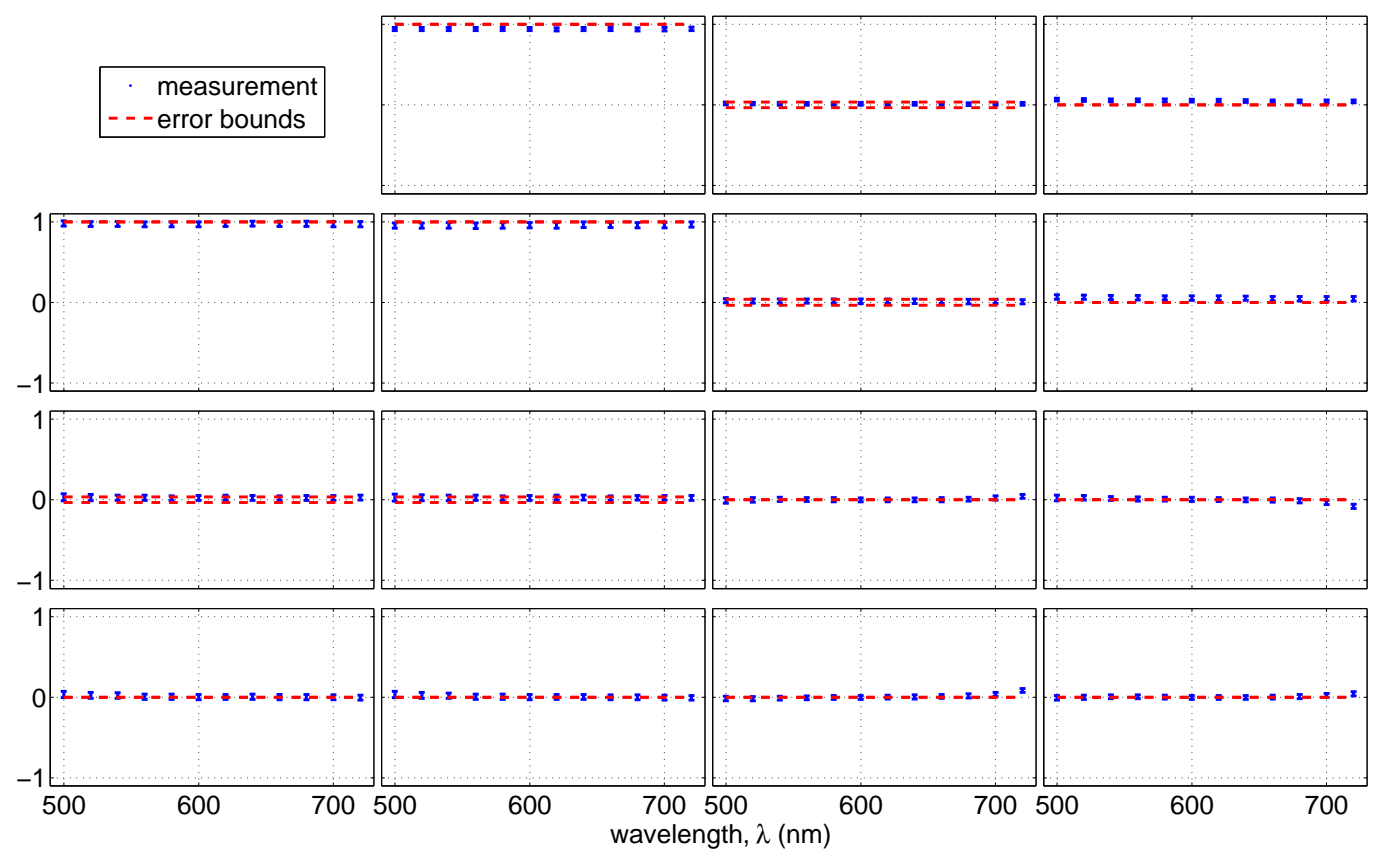

Figure 3.55: Mean and standard deviation of each polarizer Mueller matrix image as a function of wavelength. The red dashed lines represent the error bounds from the uncertainty expressed by equation (3.29).

with the error bounds from the uncertainty equation. For this case, the orientation was assumed to be $\theta=0^{\circ}$ and the error in orientation was assumed to be $\Delta \theta=2^{\circ}$. The orientation error is from the $1^{\circ}$ resolution markings on the component rotation mount. These markings result in a $1^{\circ}$ uncertainty in mounting the polarizer in the mount, and a $1^{\circ}$ uncertainty in estimating the transmission axis.

\section{Waveplates}

The waveplate measurements probe the ability of the system to measure birefringence. Both waveplates (Meadowlark Optics, NHM-100-633, NQM-100-633) have a center wavelength of $632.8 \mathrm{~nm}$, and exhibit a slightly nonlinear relationship between 
retardance and incident wavelength. According to the manufacturer, these dispersion effects produce an uncertainty of $\approx 4.5 \%$ in retardance at $\pm 10 \%$ of the center wavelength, which corresponds to wavelengths of $\approx 570-696 \mathrm{~nm}$ [121]. Additionally, the manufacturer provided information about incidence angle effects, and states that incidence angles up to $16.26^{\circ}$ can cause a change in retardance of $\approx 1.5 \%$ [121].

For each waveplate, measurements were made at wavelengths of $560-720 \mathrm{~nm}$ at $20 \mathrm{~nm}$ increments and fast axis angles of approximately $\theta \approx 0^{\circ}, 30^{\circ}$, and $60^{\circ}$. The error bounds from the uncertainty equation were also calculated in the same manner for both waveplates. The values used for $\theta$ for the three fast axis angles in the uncertainty equation were $\theta=0^{\circ}, 30^{\circ}$, and $60^{\circ}$, respectively, and for the orientation uncertainty, $\Delta \theta=2^{\circ}$. In terms of retardance, an uncertainty of $6 \%$ was used, which compensated for both wavelength and incidence angle effects. The uncertainty values for the waveplates were the result of multiplying the component retardance by $\approx 6 \%$. For the HWP, $\Delta \delta=(0.5)(0.06)=0.03$, and for the QWP, $\Delta \delta=(0.25)(0.06)=0.015$. Figures 3.56-3.58 show the mean and standard deviation of each HWP Mueller matrix image as a function of wavelength for the three fast axis orientations, along with the corresponding error bounds. Figures 3.59-3.61 show the same information as the previous three figures, but for the QWP. 


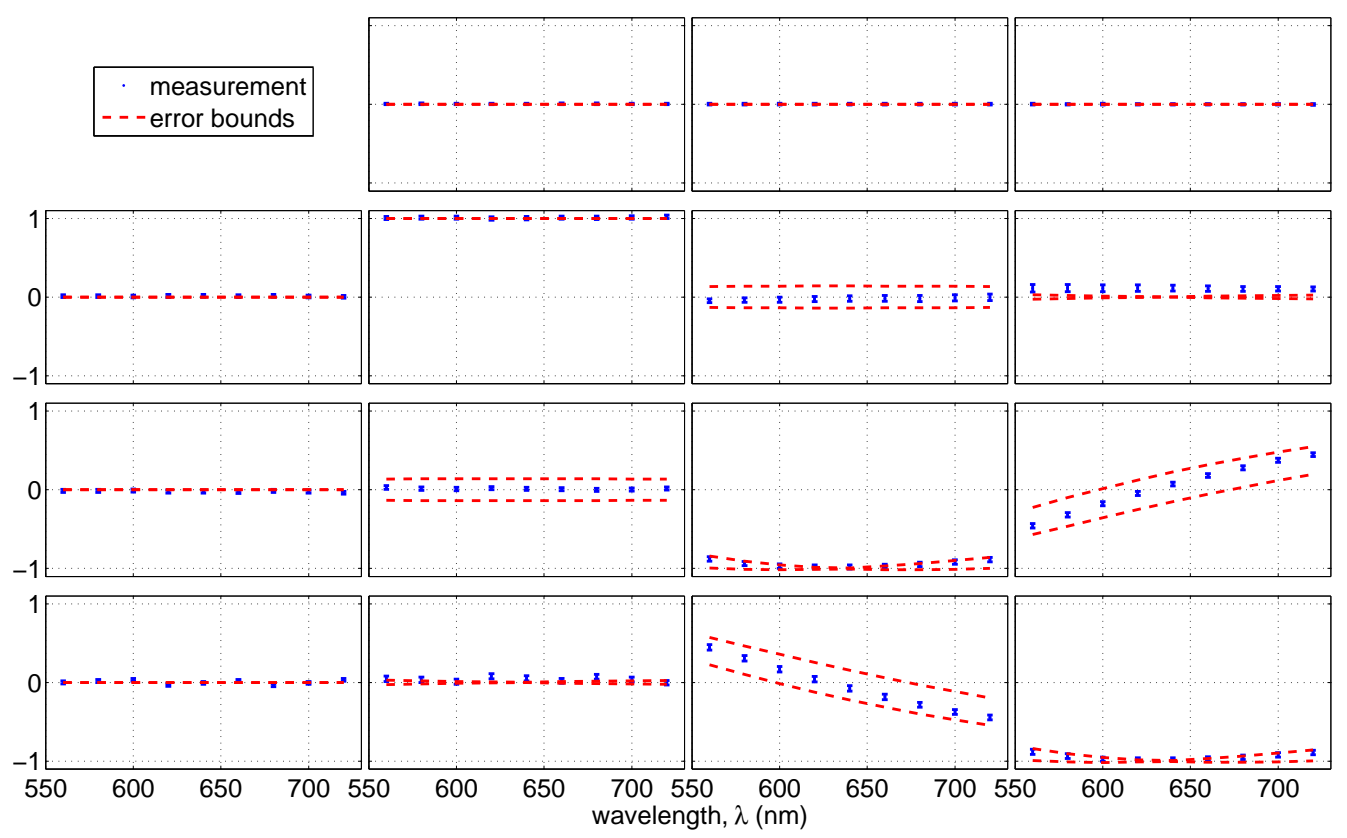

Figure 3.56: Mean and standard deviation of each HWP Mueller matrix image as a function of wavelength for a fast axis orientation of $\approx 0^{\circ}$. The red dashed lines represent the error bounds from the uncertainty expressed by equation (3.29).

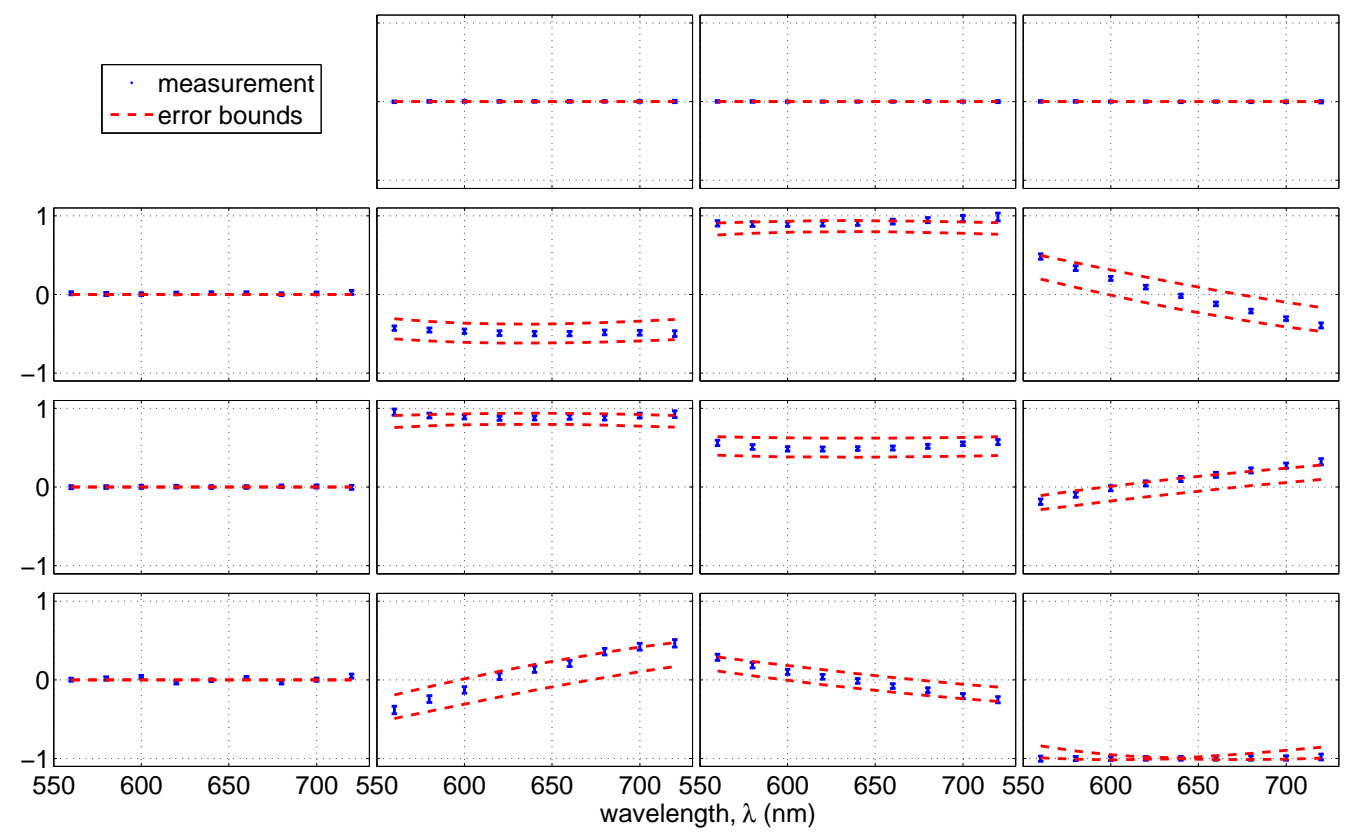

Figure 3.57: Mean and standard deviation of each HWP Mueller matrix image as a function of wavelength for a fast axis orientation of $\approx 30^{\circ}$. The red dashed lines represent the error bounds from the uncertainty expressed by equation (3.29). 


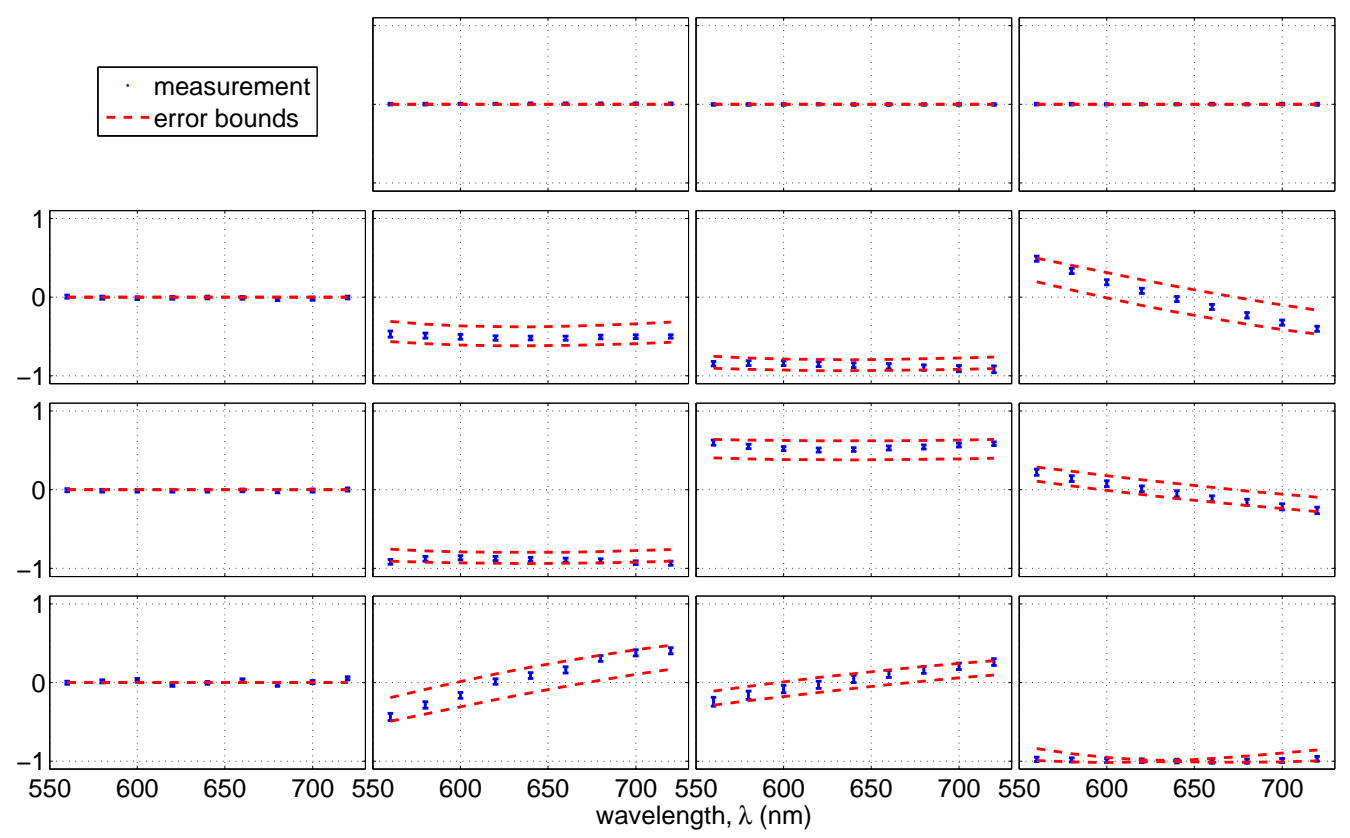

Figure 3.58: Mean and standard deviation of each HWP Mueller matrix image as a function of wavelength for a fast axis orientation of $\approx 60^{\circ}$. The red dashed lines represent the error bounds from the uncertainty expressed by equation (3.29).

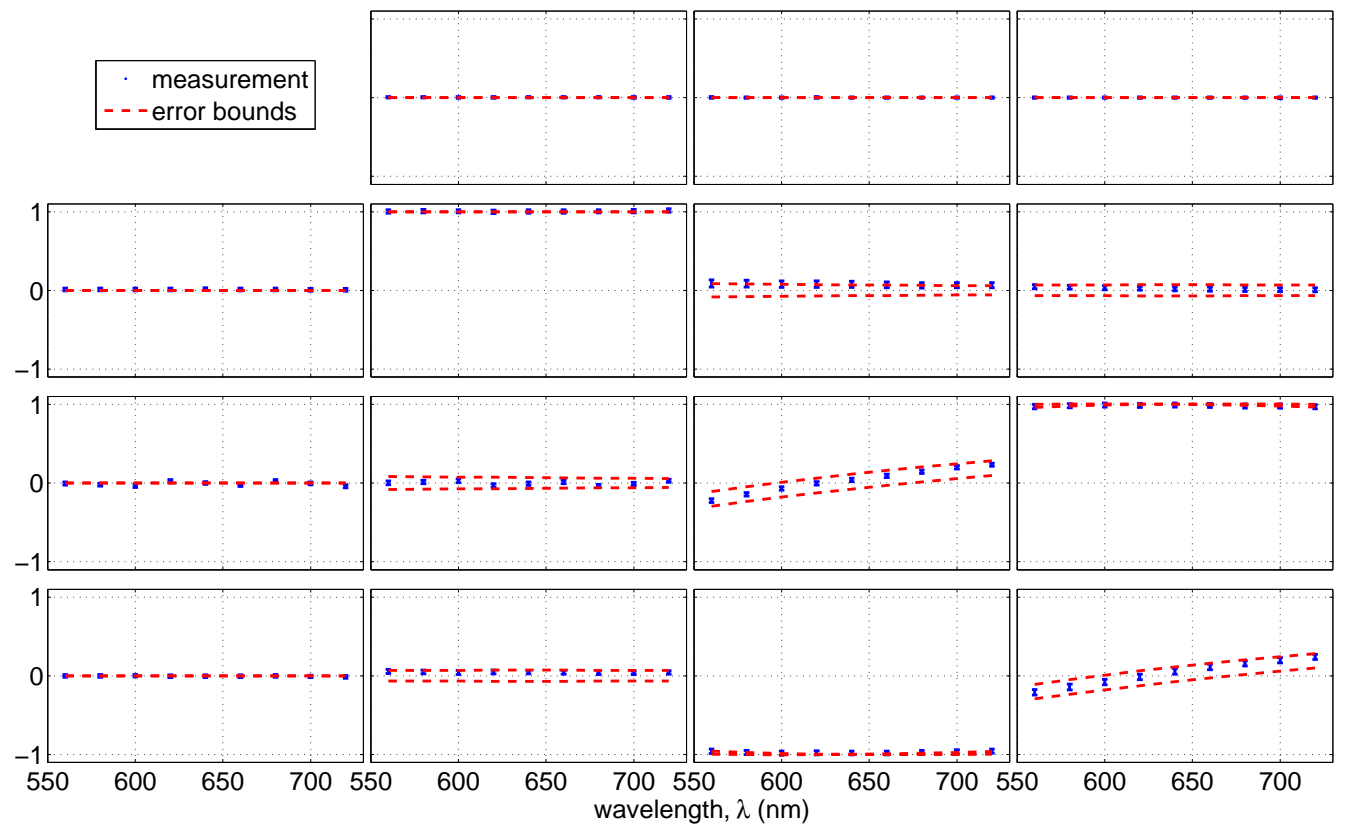

Figure 3.59: Mean and standard deviation of each QWP Mueller matrix image as a function of wavelength for a fast axis orientation of $\approx 0^{\circ}$. The red dashed lines represent the error bounds from the uncertainty expressed by equation (3.29). 


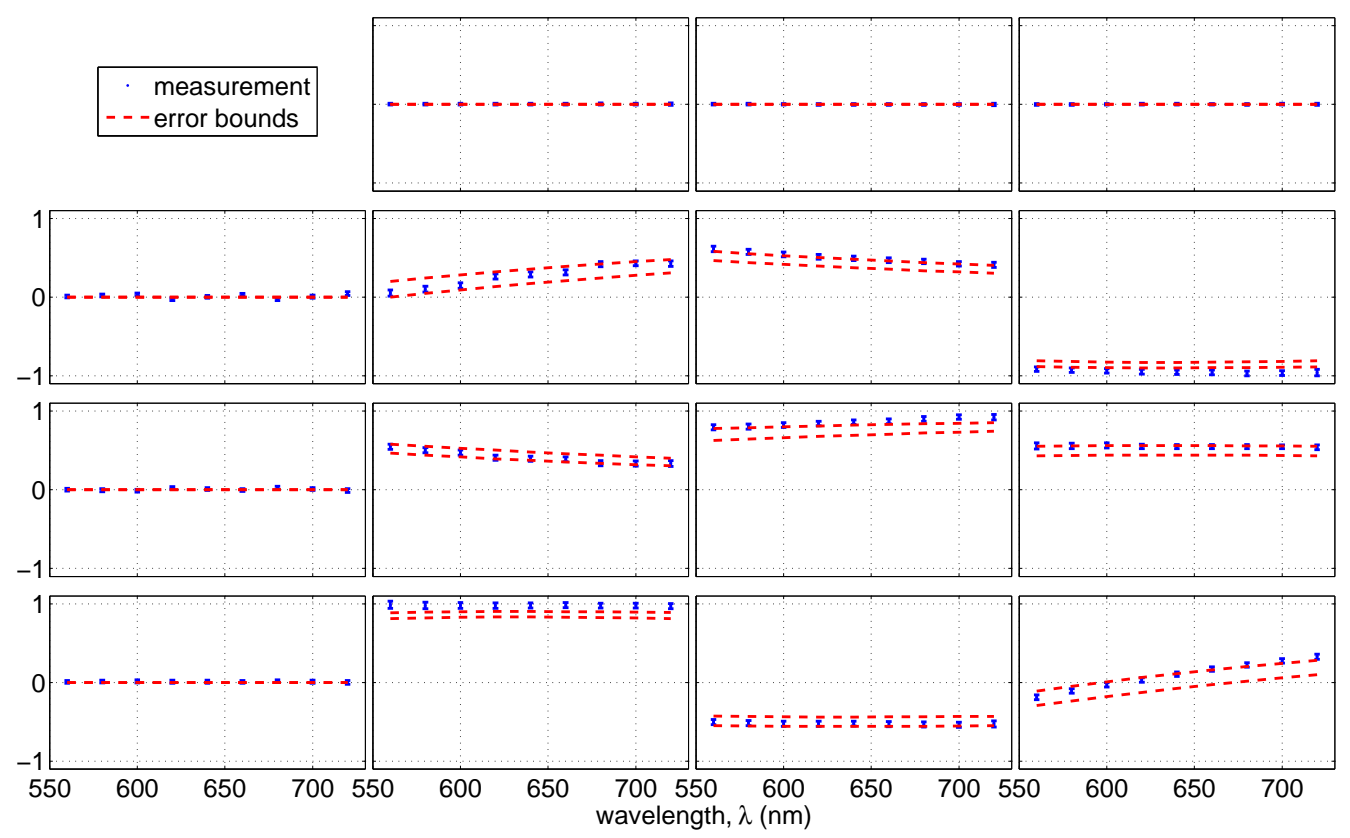

Figure 3.60: Mean and standard deviation of each QWP Mueller matrix image as a function of wavelength for a fast axis orientation of $\approx 30^{\circ}$. The red dashed lines represent the error bounds from the uncertainty expressed by equation (3.29).

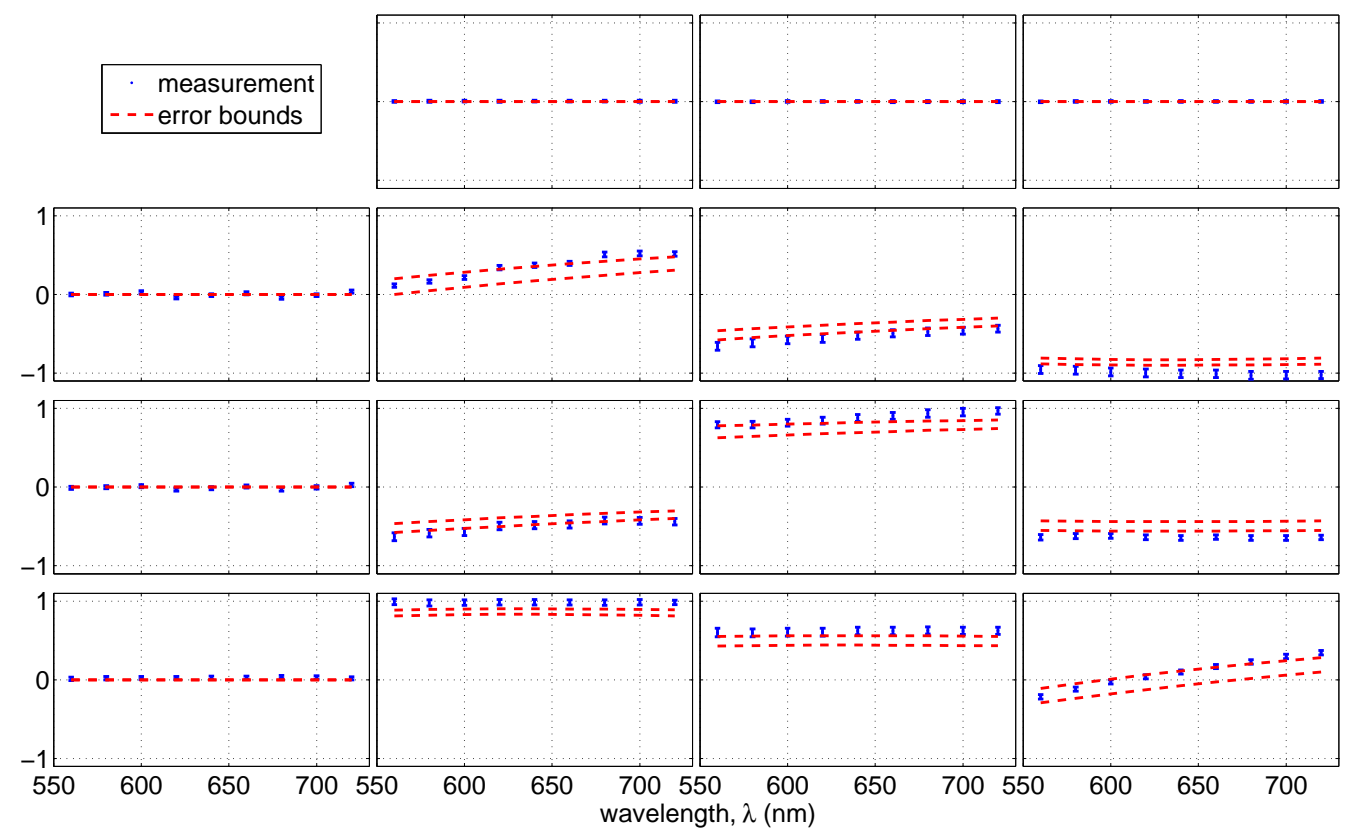

Figure 3.61: Mean and standard deviation of each QWP Mueller matrix image as a function of wavelength for a fast axis orientation of $\approx 60^{\circ}$. The red dashed lines represent the error bounds from the uncertainty expressed by equation (3.29). 


\section{Glucose mixtures}

As was the case for the optical component measurements, measurements on various glucose concentrations probe specific Mueller matrix element accuracy and sensitivity. Glucose measurements illustrate the ability of the instrument to measure optical activity, which is represented in the central $2 \times 2$ submatrix of the Mueller matrix.

Optical activity is parameterized in terms of optical rotation $\alpha$, which is a

measure (in degrees) of the rotation of the plane of polarization as it propagates through an optically active medium. Optical rotation is calculated from the expression $[122]$

$$
\alpha=[\alpha]_{\lambda}^{T} C l
$$

where $[\alpha]_{\lambda}^{T}$ is the specific rotation of the optically active species for a given temperature and wavelength with units of ${ }^{\circ}(\mathrm{g} / \mathrm{mL})^{-1} \mathrm{dm}^{-1}, C$ is the species concentration $(\mathrm{g} / \mathrm{mL})$, and $l$ is the interaction pathlength in decimeters $(\mathrm{dm})$. In terms of the Mueller matrix elements, the optical rotation is given by [122],

$$
\alpha=\frac{1}{2} \tan ^{-1}\left(\frac{m_{32}}{m_{22}}\right) .
$$

Optically active species can exhibit dispersion effects. This effect is quantified as the optical rotatory dispersion (ORD) curve, which is simply the specific rotation $[\alpha]_{\lambda}^{T}$ as a function of wavelength for a given temperature [123]. For the case of 


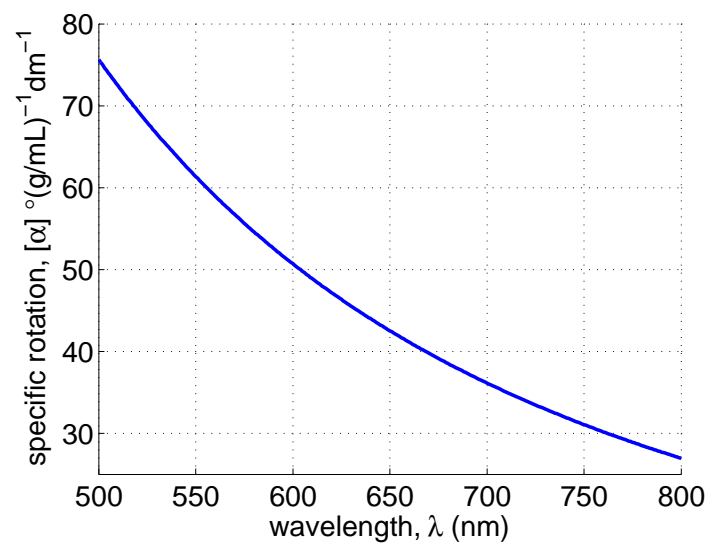

Figure 3.62: Specific rotation as a function of wavelength for glucose (ORD curve).

D-glucose, however, the specific rotation is insensitive to temperature, as there is negligible change between $0{ }^{\circ} \mathrm{C}$ and $100{ }^{\circ} \mathrm{C}$ [124]. The temperature-independent ORD curve for glucose has been estimated by Ansari et al. [125]. They estimated an analytical form of the curve from two published ORD data sets by fitting a model to the data. The analytic form is [125]

$$
[\alpha]_{\lambda}=6.3333 \times 10^{7} \lambda^{-2.1945}
$$

where Fig. 3.62 shows the ORD curve for D-Glucose from equation (3.32). The values from the ORD curve in Fig. 3.62 are used in equation (3.30) for estimating $\alpha$ at a given wavelength.

Optical activity measurements were made on three different glucose concentrations. Anhydrous D-Glucose (Fisher Scientific, D16-500) was dissolved in water for making molar concentrations of $1 \mathrm{M}, 2 \mathrm{M}$, and $3 \mathrm{M}$. The solutions were then 


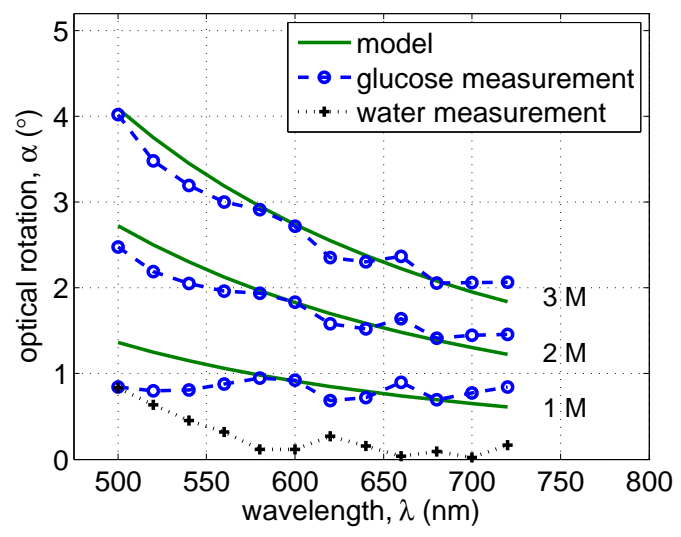

(a)

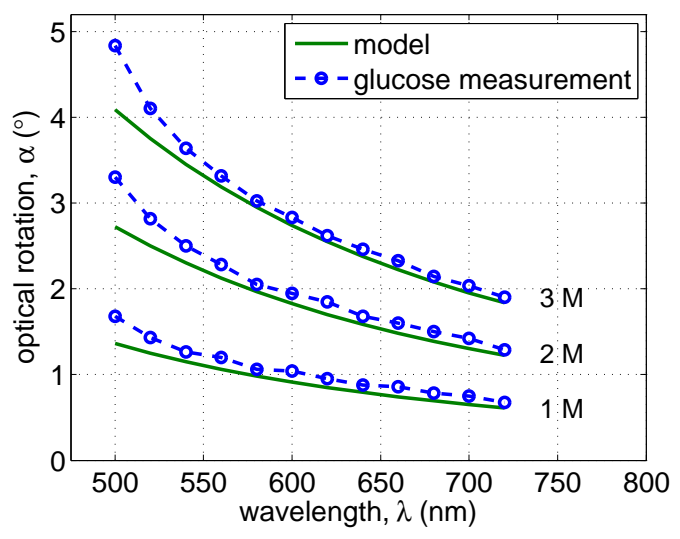

(b)

Figure 3.63: Glucose measurements represented by a) equation (3.31), b) equation $(3.33)$.

placed into a $10 \mathrm{~mm}(0.1 \mathrm{dm})$ pathlength square glass cuvette (Precision Cells Inc., 43ES10 4EA). Figure 3.63a shows the predicted and measured optical activity for the three concentrations as a function of wavelength, along with a blank measurement on water. Due to the similar structure in the glucose measurements and the water measurements, equation (3.31) was modified to compensate for this effect,

$$
\alpha=\frac{1}{2} \tan ^{-1}\left(\frac{m_{32, \text { glucose }}}{m_{22, \text { glucose }}}-\frac{m_{32, \text { water }}}{m_{22, \text { water }}}\right),
$$

where the results from this modification are shown in Fig. 3.63b.

An uncertainty analysis also was done on the glucose measurements. This analysis resulted in error bounds that reflected uncertainty in both preparing the solutions and measuring the optical activity. For example, equation (3.30) was used 
for determining the uncertainty in the preparation process, which also includes incidence angle effects. Uncertainty exists in weighing the solute and then adding the correct volume of solvent, as the concentration is $C=W / V$, where $W$ is the solute weight in grams, and $V=10 \mathrm{~mL}$ is the final volume of the solution. Thus, for the uncertainty equation, the error in weight $\Delta W$ was estimated at $5 \%$ of the total glucose weight $\Delta W=W * 0.05$, and the error in final volume was estimated to be $5 \%$, $\Delta V=0.5 \mathrm{~mL}$. Furthermore, the pathlength error can be represented as the additional pathlength caused by incidence angle effects (it was assumed that rotation of the square cuvette introduced a negligible pathlength increase). The pathlength term from equation (3.30) that was used in the uncertainty equation was $l / \cos \psi_{\text {eff. For }}$ the uncertainty equation, an effective incidence angle of $\psi_{\text {eff }}=9.38^{\circ}$ was used, with an error $\Delta \psi=6.88^{\circ}$. The effective incidence angle was computed from the effective solid angle of illumination (see Appendix H). The error value was computed from the difference between the maximum acceptance angle and the effective incidence angle, $16.26^{\circ}-9.38^{\circ}=6.88^{\circ}$

The measurement uncertainty was estimated from equation (3.31) for the regular optical activity computation, and equation (3.33) for the modified optical activity computation. This resulted in two uncertainty parameters for equation (3.31) (corresponding to Mueller matrix elements $m_{22}$ and $m_{32}$ for glucose) and four uncertainty parameters for equation (3.33) (corresponding to Mueller matrix elements $m_{32}$ and $m_{22}$ for both glucose and water). In general, the standard deviation of the Mueller 


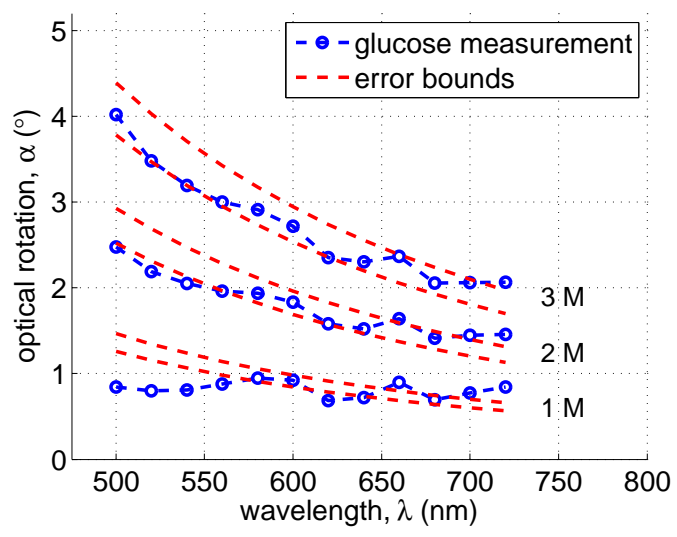

(a)

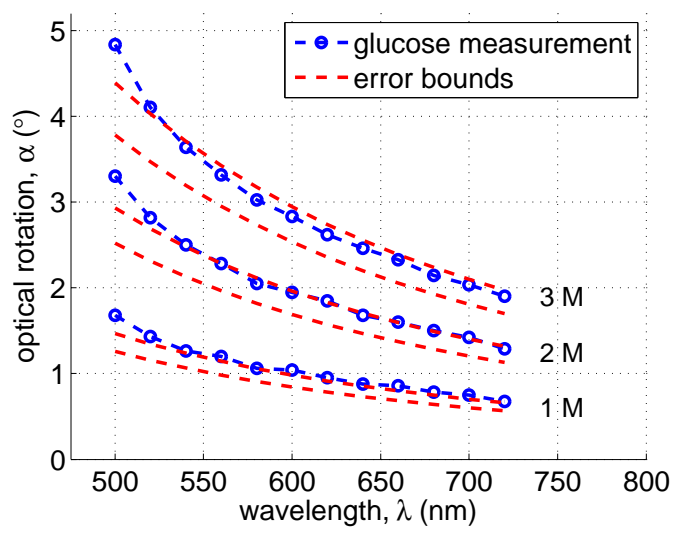

(b)

Figure 3.64: Glucose measurement error bounds for the a) regular optical activity computation, b) modified optical activity computation.

matrix images for $m_{22}$ and $m_{32}$ for both glucose and water, at all wavelengths, was $\approx 2-3 \%$, resulting in an error of $\Delta m_{22}=\Delta m_{32} \approx 0.02-0.03$

Figure 3.64a shows the error bounds and the measurement results for the three concentrations as a function of wavelength for the regular optical activity computation. The error bounds are the root sum square of the preparation process uncertainty and the measurement uncertainty. Figure 3.64b shows the same data as Fig. 3.64a, but for the modified optical activity computation of equation (3.33).

\section{Conclusions}

The results from this section show that the spectral imaging polarimeter performs accurate and sensitive transmission measurements on a variety of test samples. For example, the system is distortion-free, exhibits the desired magnification, and has 
condition numbers that approach the theoretical value in both transmission and reflection. The condition number at shorter wavelengths is higher due to the lower SNR at those wavelengths. Additionally, the structure in the condition number images at $500 \mathrm{~nm}$ in Figs. 3.48a and 3.48c could be a result of etalon effects, where reflections from multiple surfaces produce interference effects. The temporal coherence length at $500 \mathrm{~nm}$ is $\approx 35 \mu \mathrm{m}$, which is longer than the thickness of the LCVR cavities, $\approx 5-10$ $\mu \mathrm{m}$. Thus, if there is a slight misalignment in the window of an LCVR (i.e., a wedge in the LCVR), then multiple reflections could cause the interference effects.

For both configurations, the RMS error for the Mueller matrix of air is less than $3 \%$ for wavelengths in the range of $\lambda=500-720 \mathrm{~nm}$. Although the system can be calibrated accurately in both configurations, there are limitations when measuring samples in the backscatter configuration. The backscatter configuration will be discussed in more detail in Sections 5.4 and 5.5.

The next set of measurements, which explored the training set size, confirmed the simulation results. These measurements show that smaller training sets can provide calibrations that are just as accurate as larger training sets. For example, Fig. 3.54 shows that the RMS error for the entire air Mueller matrix for the 6- and 12member training sets has only $\approx 0.5 \%$ more error than the larger training sets. Using a training set with fewer members results in faster calibration times, and can allow for additional recalibrations due to temperature fluctuations or system movement. 
The system can also accurately measure polarization effects to within the inherent limitations of the system (i.e., incidence angle effects, small LCVR and sample misalignments, and small temperature uncertainties). This is illustrated by the optical component measurements, as the system can accurately recover polarization effects. For example, for the polarizer, all Mueller matrix element data fall within the uncertainty bounds except for elements $(1,2),(2,1),(2,2),(1,4)$, and $(2,4)$. These five elements all have mean error values of less than $7 \%$.

For the half-wave plate measurements, a majority of Mueller matrix element data fall within the uncertainty bounds for all fast axis orientations. Elements $(2,4)$ and $(4,2)$ at a fast axis of $0^{\circ}$ are the exceptions, and exhibit error as large as $11 \%$ and $8 \%$, respectively. For the quarter-wave plate, all Mueller matrix elements are within the uncertainty bounds only for a fast axis angle of $0^{\circ}$. At a fast axis angle of $30^{\circ}$, the elements $(2,4),(3,3)$, and $(4,2)$ are not within the uncertainty bounds, and for an orientation of $60^{\circ}$, the lower right $3 \times 3$ submatrix has data that lie outside the bounds. The elements outside the bounds for orientations of $30^{\circ}$ and $60^{\circ}$ typically have error around $5-10 \%$, but it can be as much as $13 \%$, which is the case for elements $(2,4)$ and $(4,2)$ at $60^{\circ}$. The error in the waveplate measurements could be a result of dispersion. Although it was estimated that dispersion effects produced an uncertainty of $\approx 4.5 \%$ in retardance, this value might not fully account for these effects. The waveplate model does not account for dispersion, as it assumes a linear relationship between retardance and wavelength. 
The glucose measurements exemplify the ability of the system to measure optical activity. The results show the system can reliably measure optical rotations of at least $2^{\circ}$ at wavelengths greater than $560 \mathrm{~nm}$ when using the modified optical activity computation. These measurements lie just within the uncertainty bounds.

The next chapter describes a very important propagation law in the field of optics, the van Cittert-Zernike theorem. This theorem has wide-ranging implications in the field of optics, and also is an important concept for this dissertation, as this theorem is used for interpreting LCVR scatter measurements. 


\section{Coherence theory}

\subsection{Introduction}

In the context of optics, coherence and correlation are synonymous. Coherence means correlation, and correlation means coherence. Coherence describes the statistical properties of optical fields in terms of waveform fluctuations in space and time. In general, coherence theory aims to quantify the statistical similarity between wave vibrations at either two locations or two instances in time. To achieve this goal, many mathematical constructs have been developed, including various coherence functions (for more details, see Appendix E). Typically, these constructs have been carried out in the context of scalar wave theory. This representation has led to the development of a significant scalar propagation law called the van Cittert-Zernike theorem, which establishes the link between coherence and directionality of propagation. The rest of this chapter describes the derivation of the van Cittert-Zernike theorem. 


\subsection{The van Cittert-Zernike theorem}

The generalized van Cittert-Zernike theorem defines a reciprocal Fourier transform relationship between coherence and intensity for propagation from a source to the far field [126]. The generalized theorem achieves this by quantifying two relationships. To begin, the first relationship states that the far field coherence is proportional to the Fourier transform of the source intensity. The second relationship maintains that the far field intensity pattern is proportional to the Fourier transform of the source coherence. The second relationship, commonly known as the van Cittert-Zernike theorem, is a fundamental concept of this dissertation, as it establishes a link between source coherence (i.e., structural organization) and the directionality of propagation.

Arriving at the van Cittert-Zernike theorem starts with the propagation of mutual coherence using the Huygens-Fresnel principle, and includes several additional assumptions. From Appendix E, the mutual coherence describes the cross-correlation between fields at two positions for a given time delay. The Huygens-Fresnel principal is a modification to Huygens principal, which originally stated that each point on a wavefront is considered the source of secondary wavelets, which spread out to form a new wavefront [120]. The modification incorporated by the Huygens-Fresnel principle is that each secondary source radiates with a directional amplitude pattern [20].

The assumptions that were alluded to earlier involve characteristics about the 
source, such as it being quasi-monochromatic, quasi-homogeneous, and partially coherent. Quasi-monochromatic light is defined such that its effective bandwidth $\Delta \nu$ is much smaller than its center frequency $\nu_{0}, \Delta \nu / \nu_{0} \ll 1[20]$. A partially coherent, quasi-homogeneous source has coherence properties that depend on separation distances within the source plane [20]. An additional assumption describes propagation conditions. Here, light propagates under the paraxial (small angle) approximation.

The discussion begins by invoking the Huygens-Fresnel principle for propagating a light wave with arbitrary coherence properties. A light wave with wavefront $\Sigma_{1}$ produces secondary wavelets with a directional amplitude pattern. The wavefront propagates from left to right, ultimately producing the wavefront $\Sigma_{2}$, shown in Fig. 4.1. The mutual coherence function $(\mathrm{MCF})$ on the wavefront $\Sigma_{2}$ is

$$
\Gamma\left(Q_{1}, Q_{2}, \tau\right)=\left\langle u\left(Q_{1}, t+\tau\right) u^{*}\left(Q_{2}, t\right)\right\rangle
$$

where $u$ represents the field, $\tau$ is the time delay, and the angular brackets denote an ensemble average.

Under quasi-monochromatic conditions, the fields of the two wavefronts $\Sigma_{1}$ 


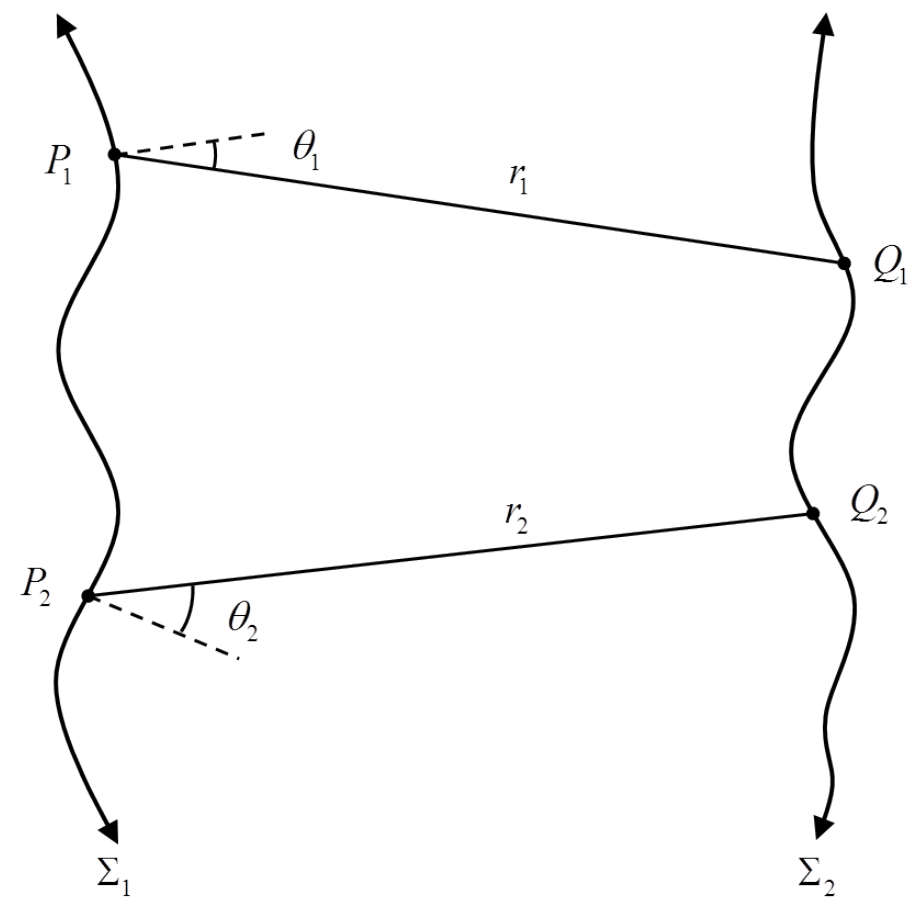

Figure 4.1: Schematic for illustrating the propagation of mutual coherence.

and $\Sigma_{2}$ are related by the Huygens-Fresnel principle as follows,

$$
\begin{aligned}
u\left(Q_{1}, t+\tau\right) & =\iint_{\Sigma_{1}} \frac{1}{i \bar{\lambda} r_{1}} u\left(P_{1}, t+\tau-\frac{r_{1}}{c}\right) \chi\left(\theta_{1}\right) \mathrm{d} S_{1} \\
u^{*}\left(Q_{2}, t+\tau\right) & =\iint_{\Sigma_{2}} \frac{1}{i \bar{\lambda} r_{2}} u\left(P_{2}, t+\tau-\frac{r_{2}}{c}\right) \chi\left(\theta_{2}\right) \mathrm{d} S_{2},
\end{aligned}
$$

where $\bar{\lambda}=c / \bar{\nu}$ is the mean wavelength, $c$ is the speed of light, $r_{1}$ is the distance from $P_{1}$ to $Q_{1}, r_{2}$ is the distance from $P_{2}$ to $Q_{2}, \theta$ is the angle between the line connecting the given pairs of points and the wavefront normal of $\Sigma_{1}$, and $\chi(\theta)$ is the obliquity factor such that $\chi(0)=1$ and $0 \leq \chi(\theta) \leq 1$. This relationship is subject to the restriction that $r \gg \bar{\lambda}[20]$. 
Substitution of the expressions in equation (4.2) into equation (4.1), and then changing the orders of integration and averaging gives

$$
\Gamma\left(Q_{1}, Q_{2}, \tau\right)=\iint_{\Sigma_{2}} \iint_{\Sigma_{1}} \frac{\left\langle u\left(P_{1}, t+\tau-\frac{r_{1}}{c}\right) u^{*}\left(P_{2}, t+\tau-\frac{r_{2}}{c}\right)\right\rangle}{\bar{\lambda}^{2} r_{1} r_{2}} \chi\left(\theta_{1}\right) \chi\left(\theta_{2}\right) \mathrm{d} S_{1} \mathrm{~d} S_{2} .
$$

By equation (4.1), the averaged term in the integrand is simply the source $\mathrm{MCF}$

$$
\Gamma\left(Q_{1}, Q_{2}, \tau\right)=\iint_{\Sigma_{2}} \iint_{\Sigma_{1}} \Gamma\left(P_{1}, P_{2}, \tau+\frac{r_{2}-r_{1}}{c}\right) \frac{\chi\left(\theta_{1}\right)}{\bar{\lambda} r_{1}} \frac{\chi\left(\theta_{2}\right)}{\bar{\lambda} r_{2}} \mathrm{~d} S_{1} \mathrm{~d} S_{2} .
$$

The mutual coherence functions of wavefronts $\Sigma_{1}$ and $\Sigma_{2}$ can be simplified by setting the time delay $\tau$ equal to zero. This is a special case [127], and the mutual coherence functions with $\tau=0$ are written as the mutual intensities,

$$
\begin{aligned}
& \Gamma\left(Q_{1}, Q_{2}, 0\right)=J\left(Q_{1}, Q_{2}\right) \\
& \Gamma\left(P_{1}, P_{2}, \frac{r_{2}-r_{1}}{c}\right)=J\left(P_{1}, P_{2}\right) \exp \left[-i \frac{2 \pi}{\bar{\lambda}}\left(r_{2}-r_{1}\right)\right] .
\end{aligned}
$$

Substituting the expressions in equation (4.5) into equation (4.4) gives the basic propagation law for mutual intensity [20],

$$
J\left(Q_{1}, Q_{2}\right)=\iint_{\Sigma_{2}} \iint_{\Sigma_{1}} J\left(P_{1}, P_{2},\right) \exp \left[-i \frac{2 \pi}{\bar{\lambda}}\left(r_{2}-r_{1}\right)\right] \frac{\chi\left(\theta_{1}\right)}{\bar{\lambda} r_{1}} \frac{\chi\left(\theta_{2}\right)}{\bar{\lambda} r_{2}} \mathrm{~d} S_{1} \mathrm{~d} S_{2}
$$




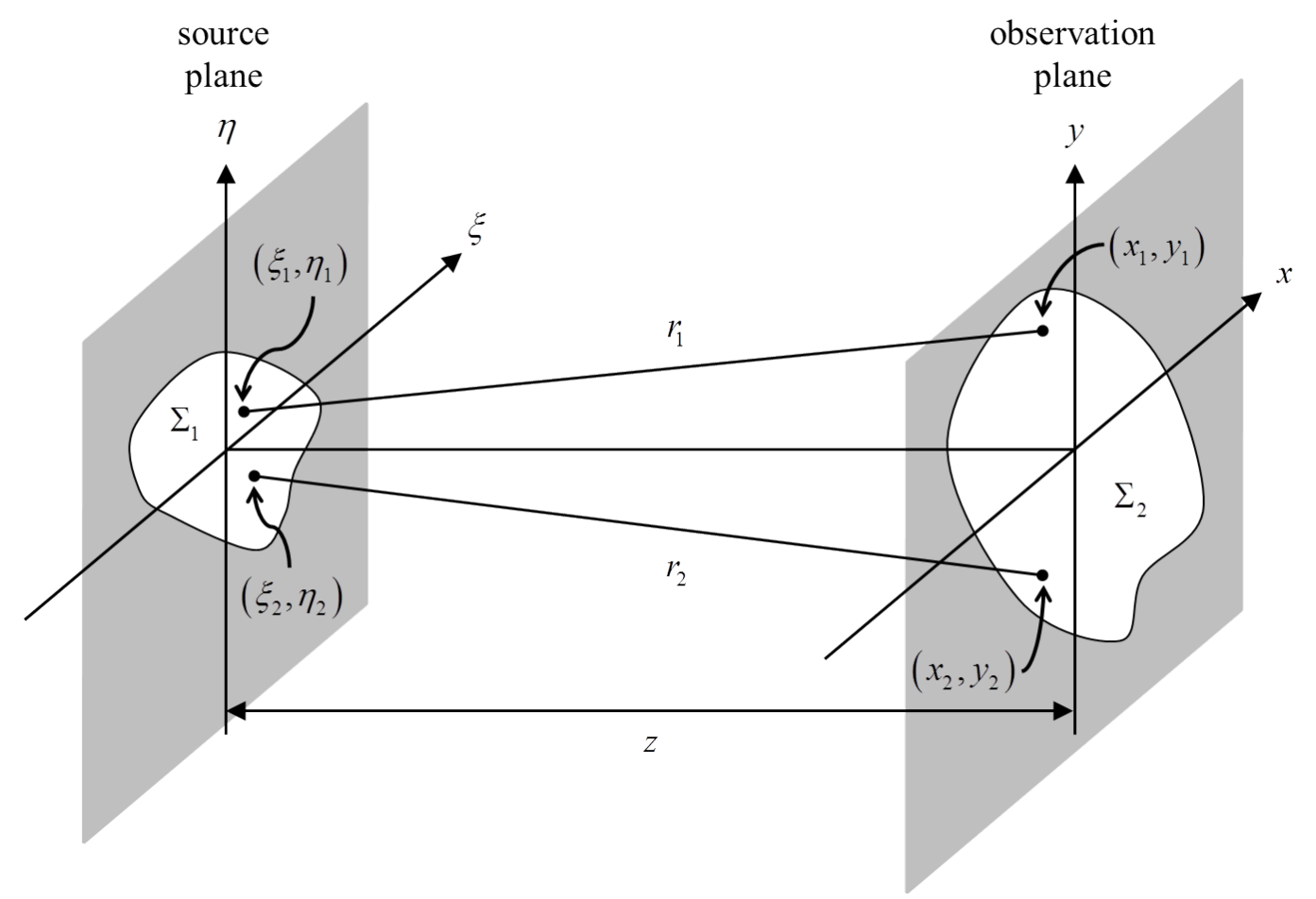

Figure 4.2: Geometry describing the van Cittert-Zernike theorem.

Arriving at the generalized van Cittert-Zernike theorem requires several additional steps. The first step consists of adopting the geometry shown in Fig. 4.2. This geometry defines a source plane described by the $(\xi, \eta)$ coordinates and an observation plane in the far field described by the $(x, y)$ coordinates. Under the paraxial approximation (i.e., $z$ is much larger than the extents of the source and observation regions, and only small angles are involved),

$$
\begin{aligned}
& \frac{1}{r_{1}} \cdot \frac{1}{r_{2}} \cong \frac{1}{z^{2}} \\
& \chi\left(\theta_{1}\right) \cong \chi\left(\theta_{2}\right) \cong 1 .
\end{aligned}
$$


To proceed further, a quasi-homogeneous model is assumed for the source mutual intensity [20],

$$
J\left(\xi_{1}, \eta_{1} ; \xi_{2}, \eta_{2}\right)=\left[I\left(\xi_{1}, \eta_{1}\right) I\left(\xi_{2}, \eta_{2}\right)\right]^{\frac{1}{2}} j(\Delta \xi, \Delta \eta)
$$

where $I$ is the source intensity at the given point, $\Delta \xi=\xi_{2}-\xi_{1}, \Delta \eta=\eta_{2}-\eta_{1}$, and $j(\Delta \xi, \Delta \eta)$ is the complex coherence factor that depends on separation distance in the source plane (from Appendix E, the complex coherence factor is the normalized version of the mutual intensity). Additionally, the coherence area is assumed to be much smaller than both the source size and any spatial structure in the source intensity distribution. This last assumption allows the source mutual intensity to be expressed as

$$
J\left(\xi_{1}, \eta_{1} ; \xi_{2}, \eta_{2}\right) \cong I(\bar{\xi}, \bar{\eta}) j(\Delta \xi, \Delta \eta)
$$

where $\bar{\xi}=\frac{\xi_{1}+\xi_{2}}{2}$ and $\bar{\eta}=\frac{\eta_{1}+\eta_{2}}{2}$. Implications of this assumption also include that $j(\Delta \xi, \Delta \eta)$ is much narrower in the $(\Delta \xi, \Delta \eta)$ plane than $I(\bar{\xi}, \bar{\eta})$ is in the $(\bar{\xi}, \bar{\eta})$ plane $[20]$.

Substituting equation (4.9) into equation (4.6) and using the paraxial conditions from equation (4.7) gives

$$
J\left(x_{1}, y_{1} ; x_{2}, y_{2}\right)=\frac{1}{(\bar{\lambda} z)^{2}} \iint_{-\infty}^{\infty} \iint_{-} I(\bar{\xi}, \bar{\eta}) j(\Delta \xi, \Delta \eta) \exp \left[-i \frac{2 \pi}{\bar{\lambda}}\left(r_{2}-r_{1}\right)\right] \mathrm{d} \xi_{1} \mathrm{~d} \eta_{1} \mathrm{~d} \xi_{2} \mathrm{~d} \eta_{2} .
$$


Using the paraxial approximation on the term $r_{2}-r_{1}$ in the exponent produces

$$
\begin{gathered}
r_{2}-r_{1} \cong \frac{1}{2 z}\left\{\left(x_{2}+x_{1}\right)\left(x_{2}-x_{1}\right)+\left(y_{2}+y_{1}\right)\left(y_{2}-y_{1}\right)+\left(\xi_{2}+\xi_{1}\right)\left(\xi_{2}-\xi_{1}\right)\right. \\
\left.+\left(\eta_{2}+\eta_{1}\right)\left(\eta_{2}-\eta_{1}\right)-2\left(x_{2} \xi_{2}-x_{1} \xi_{1}\right)-2\left(y_{2} \eta_{2}-y_{1} \eta_{1}\right)\right\} .
\end{gathered}
$$

Establishing relationships similar to those of the $(\xi, \eta)$ variables for $x$ and $y$ result in

$$
\begin{array}{ll}
\Delta x=x_{2}-x_{1}, & \bar{x}=\frac{x_{1}+x_{2}}{2} \\
\Delta y=y_{2}-y_{1}, & \bar{y}=\frac{y_{1}+y_{2}}{2} .
\end{array}
$$

Substituting equation (4.12) into equation (4.11) gives

$$
\begin{aligned}
r_{2}-r_{1} \cong \frac{1}{z}\{\bar{x} \Delta x+\bar{y} \Delta y+\bar{\xi} \Delta \xi+\bar{\eta} \Delta \eta \\
-\Delta x \bar{\xi}-\bar{x} \Delta \xi-\Delta y \bar{\eta}-\bar{y} \Delta \eta\}
\end{aligned}
$$

One final assumption results in

$$
z>4 \frac{\bar{\xi} \Delta \xi}{\lambda}, \quad z>4 \frac{\bar{\eta} \Delta \eta}{\lambda}
$$

which states that the observation distance must be much larger than the geometric mean of the largest linear source dimension and the largest linear dimension of the 
coherence area of the source [20]. This assumption permits the third and fourth terms of equation (4.13) to be dropped. Substituting equation (4.13) into equation (4.10) finally produces

$$
J\left(x_{1}, y_{1} ; x_{2}, y_{2}\right)=\frac{\kappa(\bar{x}, \bar{y}) e^{-i \psi}}{(\bar{\lambda} z)^{2}} \int_{-\infty}^{\infty} I(\bar{\xi}, \bar{\eta}) \exp \left[-i \frac{2 \pi}{\bar{\lambda} z}(\Delta x \bar{\xi}+\Delta y \bar{\eta})\right] \mathrm{d} \bar{\xi} \mathrm{d} \bar{\eta}
$$

where

$$
\begin{aligned}
\kappa(\bar{x}, \bar{y}) & =\int_{-\infty}^{\infty} j(\Delta \xi, \Delta \eta) \exp \left[i \frac{2 \pi}{\bar{\lambda} z}(\bar{x} \Delta \xi+\bar{y} \Delta \eta)\right] \mathrm{d} \Delta \xi \mathrm{d} \Delta \eta \\
\psi & =\left[\frac{2 \pi}{\bar{\lambda} z}(\bar{x} \Delta x+\bar{y} \Delta y)\right] .
\end{aligned}
$$

The expressions in equation (4.15) represent the generalized van Cittert-Zernike theorem. Equation (4.15a) describes the mutual intensity in the observation plane. This result shows that the far field coherence is proportional to the Fourier transform of the source intensity. Equation (4.15b) describes the intensity variation in the observation plane. This result states that the far field intensity pattern is proportional to the Fourier transform of the source coherence.

The physical interpretation has implications in several areas of optics. For example, the concepts of this theorem can be applied to an illuminated surface, where this surface acts as a secondary source. In this example, surface scatter can be characterized by its directionality. This directionality is proportional to the Fourier transform of the surface coherence, where the surface coherence can be described 
with a surface height correlation function. A smooth surface has a high degree of correlation and produces highly directional scatter, while a rough surface has a low degree of correlation and produces diffuse scatter. Thus, a rough surface degrades the coherence of an incident field, and the scattered fields become less directional. In general, an illuminated medium can act as a secondary source. The far field intensity that propagates from the medium is related to the correlation of the scatterers within the medium.

Another example is the propagation of light through the atmosphere. A light beam with a high degree of coherence, such as that from a laser, will degrade as it propagates through the turbulent atmosphere. This results in a beam with less coherence and less directionality due to beam spreading, beam wander, and scintillation. However, if the level of coherence of the beam is degraded before propagation, it will experience a reduced level of beam spreading, wander, and scintillation.

A final example is with respect to Köhler illumination. This illumination technique permits control of the degree of spatial coherence at the sample plane. In the Köhler configuration, a variable-diameter iris functions as the aperture stop. Manipulating the aperture stop changes the apparent angular extent of the source. Thus, the size of the aperture stop dictates the illumination coherence. See Appendix E for more details on Köhler illumination.

In summary, this chapter described the derivation of the van Cittert-Zernike 
theorem. This derivation established the link between coherence and propagation direction. In the next chapter, a specific technique for applying the van Cittert-Zernike theorem is discussed, as it is used for parameterizing LCVR structural organization. The rest of Chapter 5 provides results from structural organization measurements. 


\section{$5 \quad$ Structural organization measurements}

\section{$5.1 \quad$ Introduction}

In this chapter, a series of measurements (from the polarimeter and from a separate configuration) are presented that reveal structural organization. Polarimeter measurements include those made on a terrestrial earth rock thin section, butterfly wing scales, and a nanofiber mat. In addition to the polarimeter measurements, a second set of measurements made on LCVRs is discussed. The LCVR measurements were made with a separate configuration that employed an integrating sphere. Measurements on the LCVRs serve as a test method for interpreting structural organization measurements, as well as for determining the magnitude of scatter effects and how they impact system performance.

In addition to revealing properties about a medium, these measurements also illustrate the functionality and limitations of the system. For example, transmission measurements were made on the rock sample and emphasize the functionality of the system. Conversely, reflection measurements were made on butterfly wing scales and a nanofiber mat, and these results exposed system limitations. This section begins 
with a description of the LCVR measurements and results, followed by the polarimeter results.

\section{$5.2 \quad$ LCVR}

LCVRs are computer-controlled birefringent devices that contain nanometer-sized birefringent liquid crystals (LCs). These devices impart retardance effects through a global, uniform orientation change of the LCs, which is based on a user-defined drive voltage input. In other words, the LC structural organization dictates the device functionality. The LC structural organization also produces a specific spectral scatter component, which exhibits an inverse power law dependence. Herein is a description of a method for investigating LC structural organization by measuring the voltagedependent LC spectral scattering signature with an integrating sphere, and then relating this observable to a fractal-Born model based on the Born approximation and a von Karman spectrum. LCVR light scattering spectra are obtained at various drive voltages (i.e., different liquid crystal orientations), and then LCVR structural organization is parameterized with voltage-dependent correlation lengths. The results can aid in determining performance characteristics of systems using LCVRs, and can provide insight into interpreting structural organization measurements.

Optical devices employing liquid crystal-based technology have seen significant use recently [21]. This technology has become increasingly popular as liquid crystal 


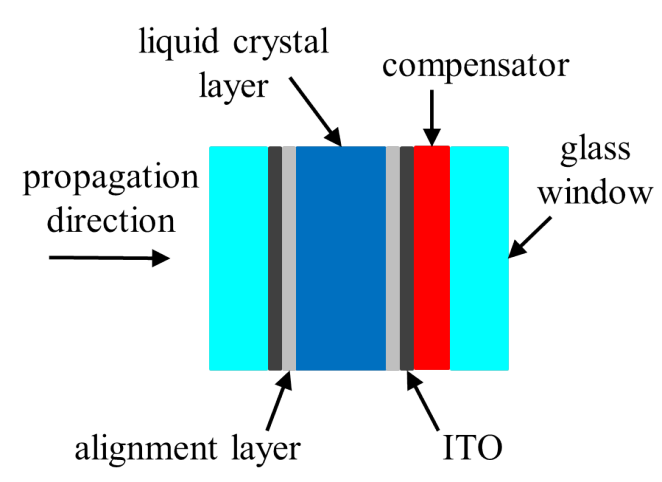

Figure 5.1: Schematic of the LCVR construction. Not to scale.

devices impart retardance effects with no mechanical movement on the millisecond time scale. One such optical device, the liquid crystal variable retarder (LCVR), has been used in various polarimetric applications, including the identification of tissue pathologies $[10,11,13]$, remote sensing $[2,14]$, and material characterization and differentiation $[16,17]$. In general, LCVRs are computer-controlled birefringent devices that contain nanometer-sized, anisotropic liquid crystals (LCs). The LCs undergo an orientation change, which ultimately dictates device functionality. LC orientation can also be thought of as a specific aspect of structural organization. Structural organization refers to the particular ordering of subwavelength scatterers, where the organization produces optical effects that are due to the form and structure of scatterer features. The pair of LCVRs used in this work, manufactured by Meadowlark Optics, achieve distinct optical effects (i.e., variable retardance and transparency) with a specific construction that controls LC structural organization.

Meadowlark Optics uses a unique fabrication process for producing variable retardance LCVRs [109]. A proprietary nematic liquid crystal mixture (called the 
liquid crystal layer in the LCVR schematic of Fig. 5.1) is sealed in a cavity several micrometers thick. Nematics are nanometer-sized, anisotropic liquid crystals, characterized as lacking positional order, but maintaining a high degree of orientational order $[108,110]$. Two parallel glass windows form the cavity, where the windows have been coated with an alignment layer and a transparent current-conducting layer, indium tin oxide (ITO). Controlling the orientation of the LCs inside the cavity is achieved by establishing an electric field across the LC layer [109]. The electric field originates from a voltage difference across the cavity, which in turn is created by electrodes attached to the windows. The electrodes deliver a square wave AC drive voltage across the liquid crystal layer, where the $\mathrm{AC}$ waveform prevents damaging ionic buildup [109]. AC waveforms are used because a DC waveform can cause the injection of supplementary charge carriers, which leads to alignment and positioning instabilities. Furthermore, DC waveforms can lead to chemical degradation of the nematic mixture [110]. Changing the magnitude of the drive voltage causes a change in the global, uniform orientation of the LCs. The modified orientation alters the effective LC birefringence and ultimately the device retardance. At low voltage, the rod-like LCs are perpendicular to the incident light, and the LCVR exhibits a higher effective birefringence. As the voltage increase, the LCs tilt towards the optical axis and the effective birefringence decreases. The birefringence does not completely vanish, however, due to surface pinning of LCs by the glass windows. Addition of a compensator, which is a fixed polymer retarder attached to the LC cell, helps the 


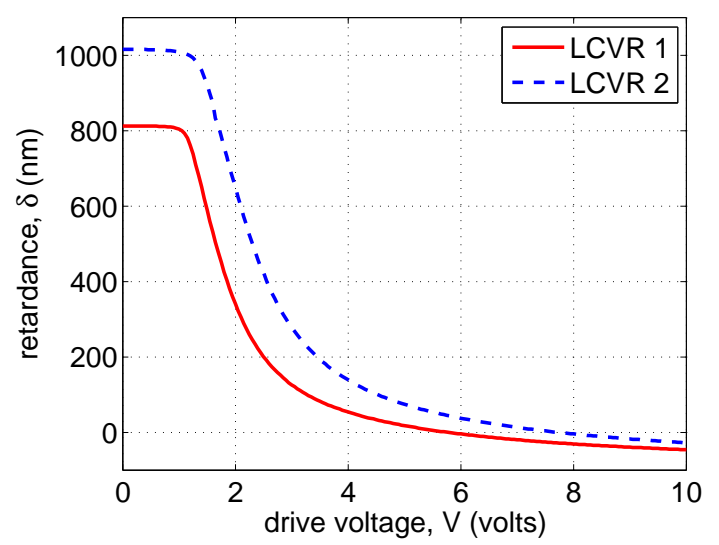

Figure 5.2: User-derived tuning curves for the two LCVRs used.

LCVR achieve zero retardance. The retardance effects imparted by these LCVRs take place on the millisecond time scale and can vary up to a full wave or more in the visible wavelengths. A user-derived tuning curve dictates the functionality for each LCVR, as the curves show the retardance as a function of drive voltage. Figure 5.2 shows the tuning curves for the LCVRs used in the measurements described herein. These curves were obtained with an $852 \mathrm{~nm}$ diode laser in separate measurements, as described in [128].

Although LCVRs provide non-mechanical polarization control and exhibit transparency, the LCs they contain scatter a small amount of light. This transparency effect is similar to that seen in the human cornea. In the cornea, the $\approx 30 \mathrm{~nm}$ diameter collagen fibrils exhibit a distinct organization that makes the cornea transparent [25]. Incident light experiences destructive interference in all scatter directions except the forward direction. Without this organization, fibril scattering would cause the cornea to be opaque. Similarly, the LC organization causes the LCVR to be transparent. 
However, a small but measurable amount of scatter is produced.

Two Meadowlark Optics nematic LCVRs are characterized by measuring the voltage-dependent LC spectral scatter and using a von Karman spectrum. The spectrum parameterizes media in terms of two variables, a correlation length $L$ and a power exponent $\alpha$. The LC scatter is measured by employing a methodology that uses an integrating sphere, and the von Karman spectrum is fit to the data. From these fits, voltage-dependent correlation lengths and power exponents are estimated. Measuring far field spectral LC scatter can reveal voltage-dependent structural organization, while also providing insight into other applications, such as determining performance characteristics of LCVR-based polarimetric systems and interpreting structural organization measurements.

\section{Theory}

LCVR transparency suggests that LCs inside the devices have a specific structural organization, where this organization dictates scatter directionality. This effect is simply a result of the van Cittert-Zernike theorem, which says the far field intensity pattern is proportional to the Fourier transform of the source coherence [20]. Here the van Cittert-Zernike theorem is used as the basis for exploring LCVR scatter, but it is restated in terms of the Born approximation. Deriving an expression for the far field intensity shows that it equals the Fourier transform of a source coherence function. This function is in the form of a correlation function obtained from a von 
Karman spectrum. The remainder of this section describes the relationship between the coherence function and far field intensity.

In studies involving weakly scattering media, the first-order Born approximation is often invoked. This approximation assumes the field inside the scattering medium equals the incident field [129]. Weakly scattering media exhibit a small relative refractive index, and for LCVRs, this is small $\left(n_{\mathrm{LC}} / n_{\text {background }} \approx 1.0-1.09\right)[111]$, thus justifying use of the Born appproximation. In this approximation, under the assumption of statistical homogeneity, the scattered intensity is

$$
I(\mathbf{q})=|U(\mathbf{q})|^{2}=\int C(\mathbf{r}) \exp (-i \mathbf{q} \cdot \mathbf{r}) \mathrm{d}^{3} \mathbf{r}
$$

where $C(\mathbf{r})$ is a spatial refractive index correlation function, $\mathbf{q}$ has a magnitude $|\mathbf{q}|=$ $2 k \sin (\theta / 2)(\theta$ is the scattering angle), and $\mathbf{r}$ is the separation distance. Equation (5.1) is similar to equation (4.15b) as both equations state that the far field intensity pattern is proportional to the Fourier transform of the source coherence. However, the two equations differ as equation (5.1) refers to a 3D secondary source, while equation (4.15b) describes a secondary planar source.

The function $C(\mathbf{r})$ is assumed to take the form of a correlation function that has a von Karman spectrum, as given by Tatarski [130]. This function describes statistically homogeneous and isotropic random media, and it depends only on a separation distance $r=\left|\mathbf{r}-\mathbf{r}^{\prime}\right|$. According to Tatarski, the correlation function has 
the form

$$
C(r)=\frac{a^{2}}{2^{\nu-1} \Gamma(\nu)}\left(\frac{r}{L}\right)^{\nu} K_{\nu}\left(\frac{r}{L}\right)
$$

where $a$ is a scaling constant, $r$ is the separation distance, $L$ is the correlation length, $K_{\nu}$ is the modified Bessel function of the second kind and order $\nu$, and $\Gamma$ is the gamma function.

Tatarski's correlation function in equation (5.2) has been interpreted as a selfaffine correlation function for describing media with small refractive index fluctuations $[131,132]$. Equation (5.2) can be approximated as

$$
C(r) \propto\left(\frac{r}{L}\right)^{H} K_{H}\left(\frac{r}{L}\right)
$$

where $H$ is the Hurst parameter, $L$ is the fractal upper scale (i.e., a correlation length), and $K_{H}$ is the modified Bessel function of the second kind and order $H$. The Hurst parameter describes the fractal nature of the medium and has values on the interval $0<H<1[131,132]$. It has been described as a measure of the refractive index distribution roughness [132].

Substituting equation (5.3) into equation (5.1) yields a proportionality expression for the scattered intensity $[131,132]$,

$$
I(q) \propto \frac{2^{H+1} \pi^{3 / 2} \Gamma\left(\frac{3}{2}+H\right) L^{D_{E}}}{\left[1+(q L)^{2}\right]^{\alpha}},
$$


where $\alpha$ is the power exponent. The exponent $\alpha$ is

$$
\alpha=H+\frac{D_{E}}{2}
$$

where $H$ is the Hurst parameter and $D_{E}$ is the Euclidean dimension of the scatterers. The Euclidean dimension can have values of $D_{E}=1,2$, or 3 , corresponding to filamentous, sheetlike, or bulk scatterers, respectively [131,133]. Ultimately, $\alpha$ can be related to the fractal dimension $D$ of a self-affine system through the realtionships $D=D_{E}+1-H=1.5 D_{E}+1-\alpha[133]$, which will be addressed later in this chapter.

A particular region of the von Karman spectrum is used in equation (5.4), where the fractal upper scale is larger than the wavelength $q>1 / L$. This reduces equation (5.4) to an expression that exhibits inverse power law behavior,

$$
I(q) \propto(q L)^{-2 \alpha}
$$

Equation (5.6) is fit to the voltage-dependent scattered spectra from the LCVR. Here, spectra are defined as the wavelength-dependent scatter data for a series of drive voltages. The fitting routine, implemented in MATLAB, produces two parameters. The first parameter, $L$, is a correlation length that describes the outer scale. The second parameter is the exponent from the fit, from which $\alpha$ is determined. 


\section{Measurements}

The forward hemispheric spectral scatter $F_{R}$ was measured from two different LCVRs using a system that consisted of a liquid crystal tunable filter (LCTF) and an integrating sphere. Because of the spectral and angular dependence in the wave vector $q$, these two devices were selected for measuring the intensity as a function of wavelength for a fixed scatter angle. The integrating sphere provided a measurable signal by collecting the forward hemispheric scatter, while the LCTF allowed for wavelength discrimination. Similarly to the LCVRs, the LCTF exhibited a scatter component. Consequently, a technique was developed for isolating LCVR scatter from LCTF scatter. The rest of the current section describes the measurement configuration, procedure, and technique for isolating LCVR scatter.

\section{Configuration}

Figure 5.3 illustrates the configuration for measuring LCVR forward hemispheric spectral scatter. The condenser portion of the system was based on Köhler illumination [114], which controls the lateral extent of the illumination pattern and the size of the illumination cone at the sample plane. The configuration consisted of a broadband source (Dolan-Jenner DC950 illuminator, 150 Watt, $6 \mathrm{~mm}$ diameter fiber), followed by a $25 \mathrm{~mm}$ focal length lens, and then an iris. The iris acted as a field stop and controlled the size of the illumination region. Another lens followed 


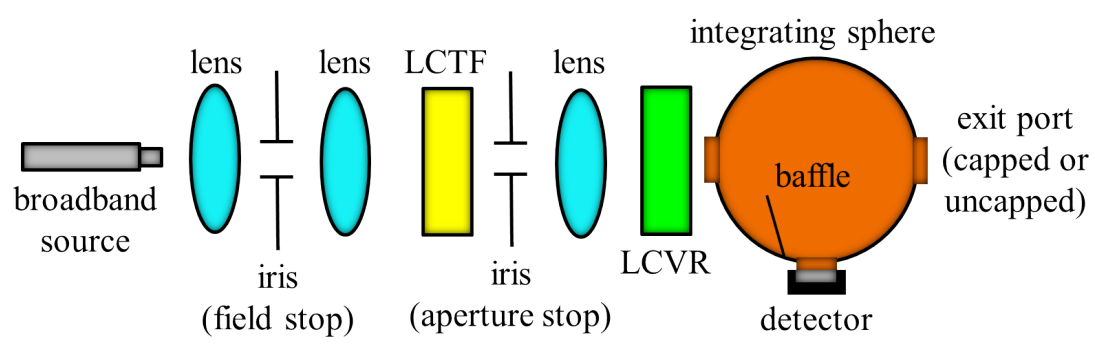

Figure 5.3: Schematic of the LCVR spectral scatter measurements.

the field stop, with a focal length of $170 \mathrm{~mm}$. After the second lens was a VariSpec LCTF (Cambridge Research \& Instrumentation, Inc., $7 \mathrm{~nm}$ bandwidth and $20 \mathrm{~mm}$ diameter clear aperture) for wavelength selection. Because of the LCTF construction (i.e., based on a Lyot filter), it transmitted linearly polarized light. For the current configuration, the LCTF was oriented such that it transmitted horizontally polarized light, parallel to the $x$-axis of the laboratory reference frame. The ensuing element after the LCTF was another iris, which served as the aperture stop. This iris specified the size of the illumination cone at the sample plane. Next in the configuration was a $200 \mathrm{~mm}$ focal length lens, followed by an LCVR (Meadowlark Optics, LRC-200, clear aperture of $17.8 \mathrm{~mm}$ ) and the integrating sphere (Labsphere, 6-inch diameter, with 1-inch entrance and exit ports).

The LCVR was placed in direct contact with the entrance port of the integrating sphere. The integrating sphere exit port can be capped, depending on the desired measurement content (i.e., measuring either scattered light or both scattered and transmitted light). Additionally, the sphere had a side port where a detector was placed for measuring the intensity. The detector used was an Ophir NOVA II 
photodiode (model PD300-BB-SH), with a $10 \times 10 \mathrm{~mm}$ sensing area. A MatLaB routine controlled the LCTF, LCVR, and data acquisition. The field stop iris diameter was decreased until the detected intensity reached a constant value. This ensured that only scattered light was captured with the integrating sphere, and that all of the unscattered light escaped. The final beam exiting the integrating sphere had a diameter of approximately $17 \mathrm{~mm}$. Finally, the geometry of the integrating sphere resulted in forward scatter within $\theta= \pm 4.8^{\circ}$ escaping the integrating sphere, thereby capturing scatter from $\theta=4.8^{\circ}-90^{\circ}$.

\section{Procedure}

LCVR scatter measurements were made on two different LCVRs, with each LCVR mounted at three different fast axis orientations $\left(0^{\circ}, 90^{\circ}\right.$, and $\left.135^{\circ}\right)$ with respect to the $x$-axis of the laboratory reference frame. LCVR 1 had a temperature control mechanism, and it was kept at a constant temperature of $22{ }^{\circ} \mathrm{C}$. The temperature of LCVR 2 was at ambient, $\approx 20{ }^{\circ} \mathrm{C}$. The LCTF was cycled through a wavelength range of $\lambda=460-720 \mathrm{~nm}$ in increments of $20 \mathrm{~nm}$. At each wavelength, the LCVR was cycled through a voltage range of $V=0-10$ volts in 0.1 volt increments.

\section{Isolating LCVR scatter}

Because the LCTF and LCVR both scattered light, it was necessary to develop a technique for isolating the LCVR scatter. This approach consisted of identifying the 
optical effects produced by the pair of liquid crystal devices (i.e., scatter and transmission components), determining and interpreting the meaning of the appropriate integrating sphere measurements, and then relating the optical effects and measurements in a manner that produced the LCVR scatter component. Seven optical effects were identified, and it was determined that four integrating sphere measurements were necessary for measuring the seven effects. After relating the seven effects to the four integrating sphere measurements, an expression was obtained for the LCVR scatter $F_{R}$

The seven optical effects produced by the LC devices were described in terms of the source intensity $I_{0}(\lambda)$, the spectral transmittances of the LCTF $\left(T_{F}(\lambda)\right)$ and LCVR $\left(T_{R}(\lambda)\right)$, and the fractional spectral scatter of the LCTF $\left(F_{F}(\lambda)\right)$ and LCVR $\left(F_{R}(\lambda)\right)$. These parameters were multiplied together in specific combinations for representing the seven transmitted and scattered components. For example, the quantity corresponding to transmission through both the LCTF and LCVR is $I_{0}(\lambda) T_{F}(\lambda) T_{R}(\lambda)$. Figure 5.4 shows the seven quantities associated with the transmitted and scattered components of both the LCTF and LCVR, while Table 5.1 describes them.

The four integrating sphere measurements combined two integrating sphere states (capped and uncapped) and two device arrangements (with and without the LCVR). Figure 5.5 illustrates the basic idea behind the four measurement configurations (denoted as $A(\lambda), B(\lambda), C(\lambda)$, and $D(\lambda)$ ), and shows the numbers corresponding to the measured components for each configuration. Measurement $A(\lambda)$ 


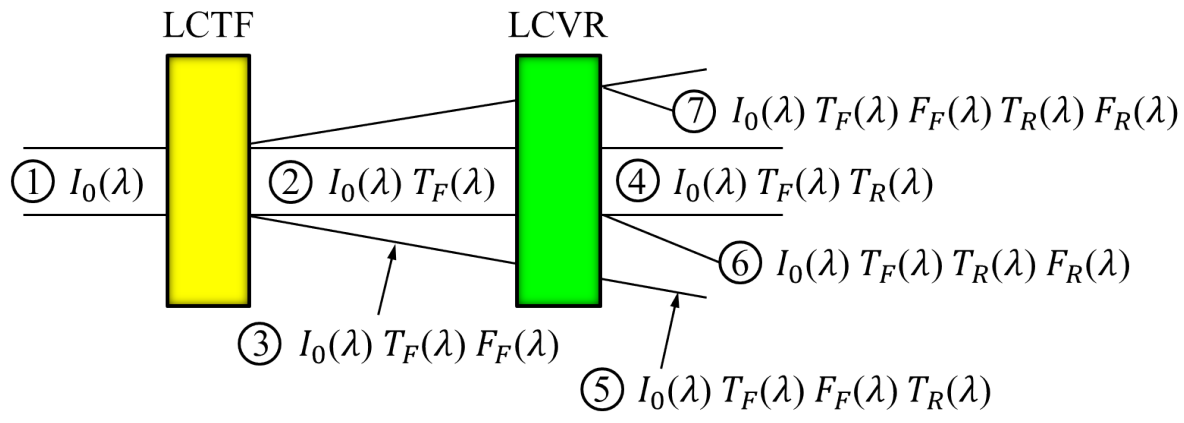

Figure 5.4: Illustration of the seven transmitted and scattered components associated with the LCTF and LCVR.

Table 5.1: Description of the seven transmitted and scattered components associated with the LCTF and LCVR.

\begin{tabular}{|c|c|}
\hline Component & Description \\
\hline 1 & Incident light \\
\hline 2 & Transmitted by LCTF \\
\hline 3 & Scattered by LCTF \\
\hline 4 & Transmitted by both LCTF \& LCVR \\
\hline 5 & Scattered by LCTF, transmitted by LCVR \\
\hline 6 & Transmitted by LCTF, scattered by LCVR \\
\hline 7 & Scattered by both LCTF \& LCVR \\
\hline
\end{tabular}



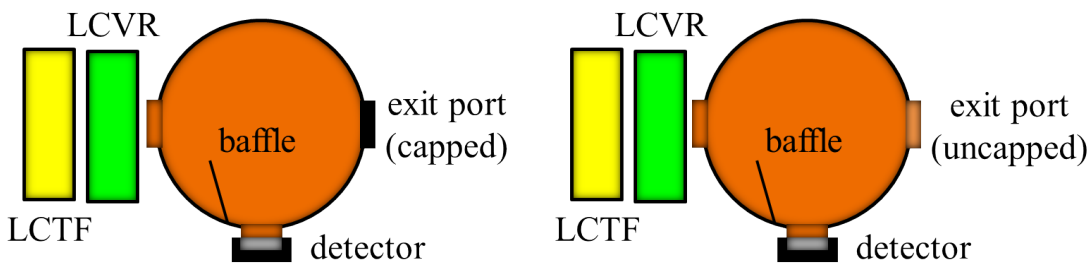

Measurement $\boldsymbol{A}(\boldsymbol{\lambda})$

$(4,5,6,7)$

Measurement $C(\lambda)$

$(5,6,7)$
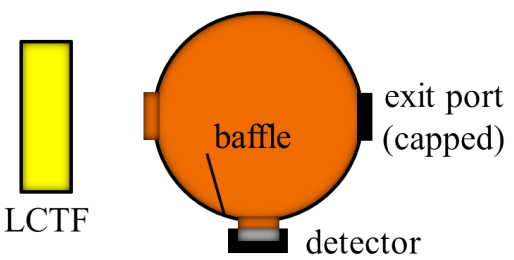

Measurement $B(\lambda)$

$(2,3)$

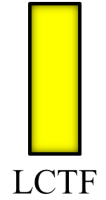

Measurement $D(\lambda)$

(3)

Figure 5.5: Basic schematics of the four measurement configurations for isolating LCVR scatter. In parentheses are the numbers corresponding to the measured components for each configuration.

was performed with the LCVR inserted in the system and the integrating sphere capped, while measurement $B(\lambda)$ was performed with the LCVR removed and the integrating sphere capped. Next, measurement $C(\lambda)$ employed a configuration with the LCVR and with the integrating sphere uncapped, and finally, measurement $D(\lambda)$ had the LCVR removed and the integrating sphere uncapped. In terms of measurement $D(\lambda)$, only a single component was captured. This was component three, where the measurement can be described by

$$
I_{0}(\lambda) T_{F}(\lambda) F_{F}(\lambda)=D(\lambda) .
$$


It is now possible to relate the four measurements in Fig. 5.5 to the seven components in Table 5.1. Each component in Table 5.1 contains the $I_{0}(\lambda)$ term. Because the ratios of the measurements $A(\lambda), B(\lambda), C(\lambda)$, and $D(\lambda)$ were used, the $I_{0}(\lambda)$ term eventually cancels out. Instead of carrying the $I_{0}(\lambda)$ term through, it and the wavelength dependence were dropped for simplicity. Equation (5.7) now becomes

$$
T_{F} F_{F}=D
$$

Similar expressions can be obtained for components two and four. Combining the expressions for components two and three gives the LCTF fractional spectral scattering component $F_{F}$,

$$
F_{F}=\frac{D}{B-D}
$$

Measurement $C$ can be expressed as the total amount of scattering from the LCTF and LCVR,

$$
T_{F} F_{F} T_{R}+T_{F} T_{R} F_{R}+T_{F} F_{F} T_{R} F_{R}=C .
$$

After substituting the expression for component four and $F_{F}$ into equation (5.10) and rearranging, the fractional spectral scattering component of the LCVR is

$$
F_{R}=\frac{\frac{C}{A-C}-\frac{D}{B-D}}{\frac{D}{B-D}+1}=\frac{\frac{C}{A-C}-F_{F}}{F_{F}+1} .
$$




\section{Results}

The LCVR fractional scattering component $F_{R}(V, \lambda)$ from equation (5.11) represents the scattered intensity on the left-hand side of equation (5.6). This component also represents scatter from the device as a whole, including scatter from the compensator, glass windows, and LCs that are pinned against the windows. In order to isolate scatter strictly from LCs undergoing rotation, we substract the value of the scattering component at $V=10$ volts from the fractional scattering component, $F_{R}(V, \lambda)-$ $F_{R}(10, \lambda)$. At $V=10$ volts, LCs that are not pinned are approximately parallel to the optical axis, thus minimizing their scatter contribution. Figure 5.6 shows the LCVR fractional scattering component on a $\log$ scale, $\log _{10}\left(F_{R}(V, \lambda)-F_{R}(10, \lambda)\right)$, for the two different LCVRs at the three fast axis orientations of $0^{\circ}, 90^{\circ}$, and $135^{\circ}$. All six elements of Fig. 5.6 are on the same scale.

Next, the $q$ parameter was determined for fitting the model in equation (5.6) to each voltage-dependent spectrum in Fig. 5.6. The $q$ parameter contains a specific scatter angle $\theta$, but the integrating sphere captured a range of angles, $\theta=4.8^{\circ}-$ $90^{\circ}$. Therefore, it was necessary to ensure that the model accurately represented the measurements. To address this, separate goniometric measurements were made (data not shown) for selecting an effective scatter angle. In these goniometric measurements, an LCVR was illuminated with a $633 \mathrm{~nm}$ HeNe laser, and the angular scatter was measured at discrete angles from $0.5^{\circ}-14^{\circ}$. From this angular scatter, a phase 
LCVR 1 at $0^{\circ}$

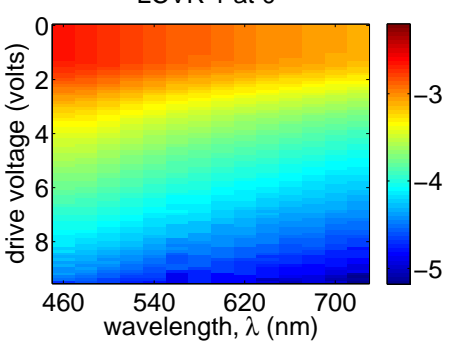

LCVR 2 at $0^{\circ}$

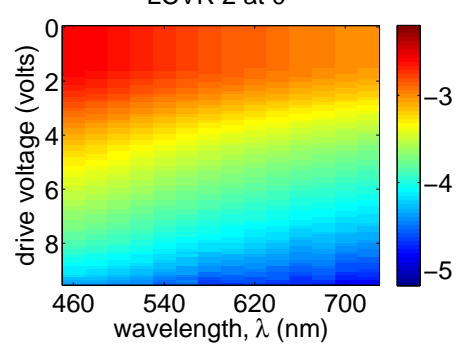

LCVR 1 at $90^{\circ}$

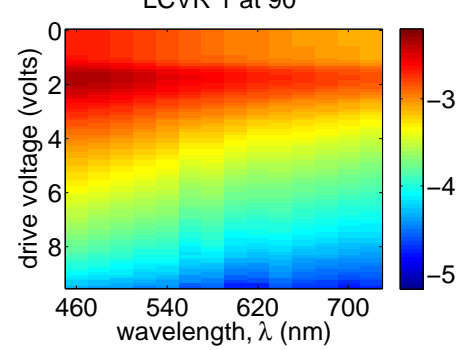

LCVR 2 at $90^{\circ}$

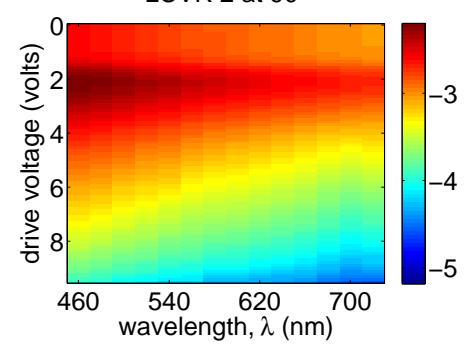

LCVR 1 at $135^{\circ}$

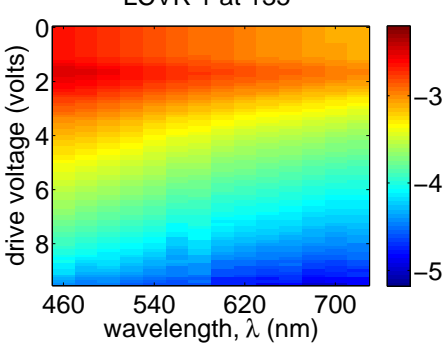

LCVR 2 at $135^{\circ}$

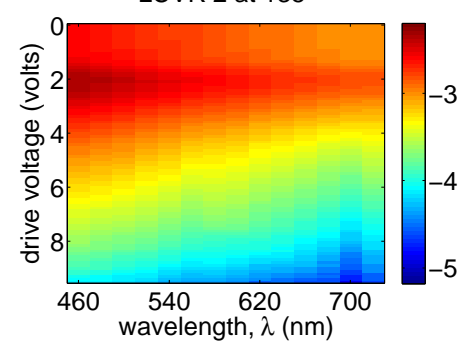

Figure 5.6: Fractional scattering component $\log _{10}\left(F_{R}(V, \lambda)-F_{R}(10, \lambda)\right)$ as a function of drive voltage and wavelength for the two LCVRs at the three fast axis orientations. This figure shows how the scattered intensity varies with drive voltage, wavelength, and fast axis orientation, and also the repeatability of the measurements.

function and then an effective scatter angle were estimated. The effective scatter angle was determined by calculating the expected value of the phase function from $\theta=4.8^{\circ}-90^{\circ}$, given by $\theta_{e f f}=\int_{4.8^{\circ}}^{90^{\circ}} \theta P_{H G}(\theta) \mathrm{d} \theta$, where $P_{H G}(\theta)$ is the HenyeyGreenstein phase function. This resulted in $\theta_{\text {eff }}=5.8^{\circ}$. With an estimate for $q$, the model in equation (5.6) was fit to each voltage-dependent spectrum in Fig. 5.6. Figure 5.7 shows sample results from the fitting routine. In it are four individual scattering spectra at different drive voltages and their respective fits to the model for LCVR 2 at a fast axis of $90^{\circ}$. These fits are representative of the fits for both LCVRs at all fast axis orientations.

From the model fits, the correlation length $L$ and the power exponent $\alpha$ were 


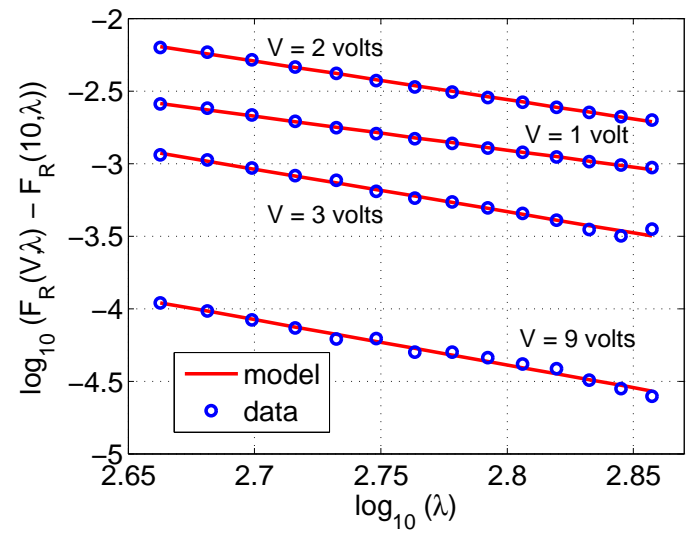

Figure 5.7: Four individual scattering spectra at various drive voltages and their respective model fits to Eq. 5.6 for LCVR 2 at a fast axis of $90^{\circ}$. This figure qualitatively shows the accuracy with which the model represents the measurement.

obtained as a function of drive voltage. In order to establish a direct link to organizational effects, the drive voltage was converted to the LC tilt angle $\beta(V)$, calculated according to [111]

$$
\beta(V)=\cos ^{-1}\left(\frac{\delta_{L C}(V)}{\delta_{\max }}\right)^{1 / 2}
$$

where $\delta_{L C}(V)$ represents the voltage-dependent retardance of the liquid crystal layer, and $\delta_{\max }$ is the maximum retardance of the LCVR. The LC tilt angle is plotted in Fig. 5.8, and is defined such that at a drive voltage of $V=0$ volts, the tilt angle is $0^{\circ}$, and then increases with increasing drive voltage. The next figure, Fig. 5.9, shows the correlation length $L$ as a function of LC tilt angle for LCVRs 1 and 2 at the three fast axis orientations. Figure 5.10 shows the power exponent $\alpha$ for LCVRs 1 and 2 at the three fast axis orientations as a function of LC tilt angle. 


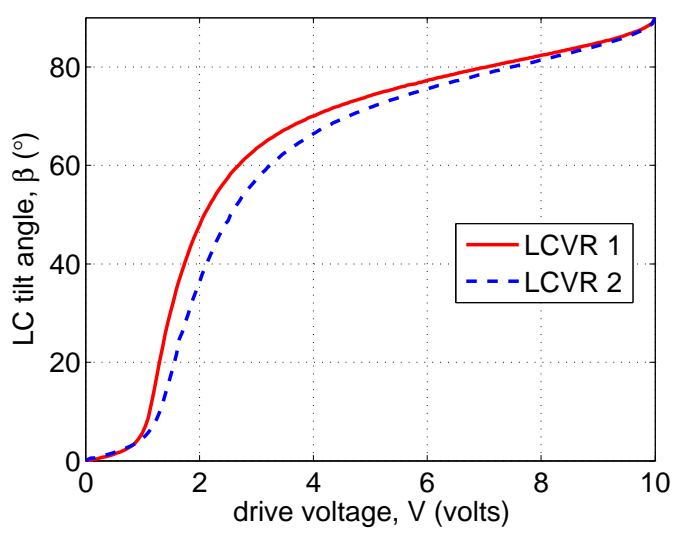

Figure 5.8: Liquid crystal tilt angle as a function of drive voltage.
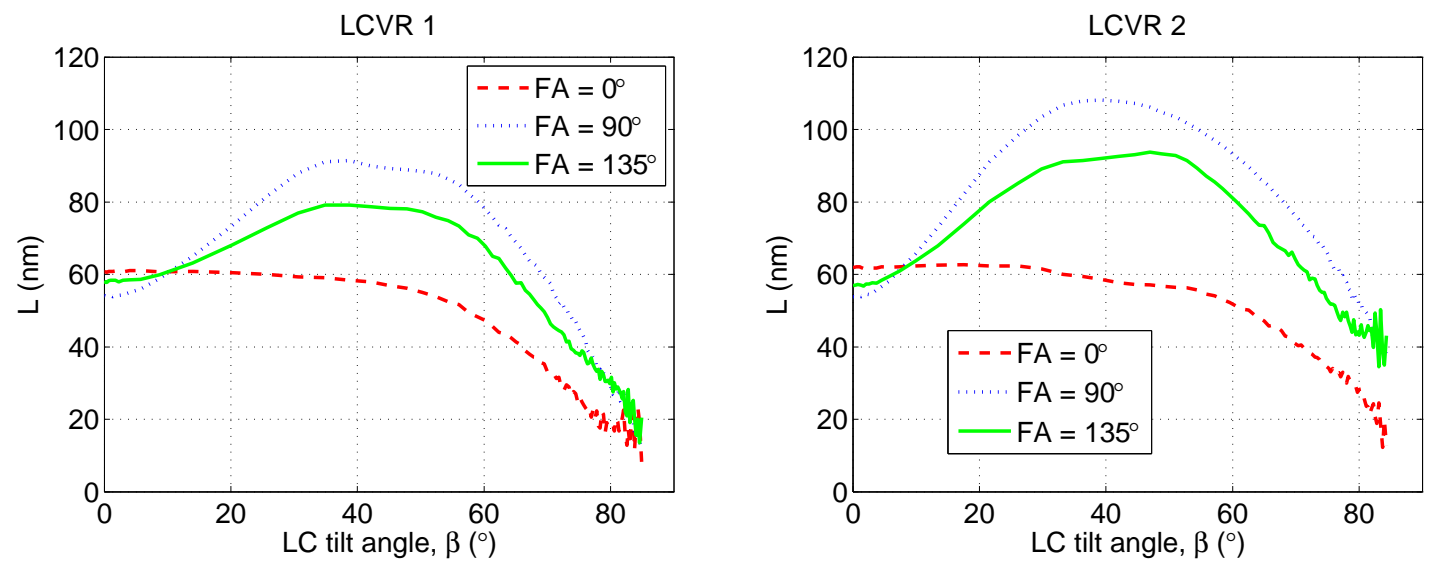

Figure 5.9: Correlation length $L$ as a function of tilt angle for the two LCVRs at the three fast axis orientations.
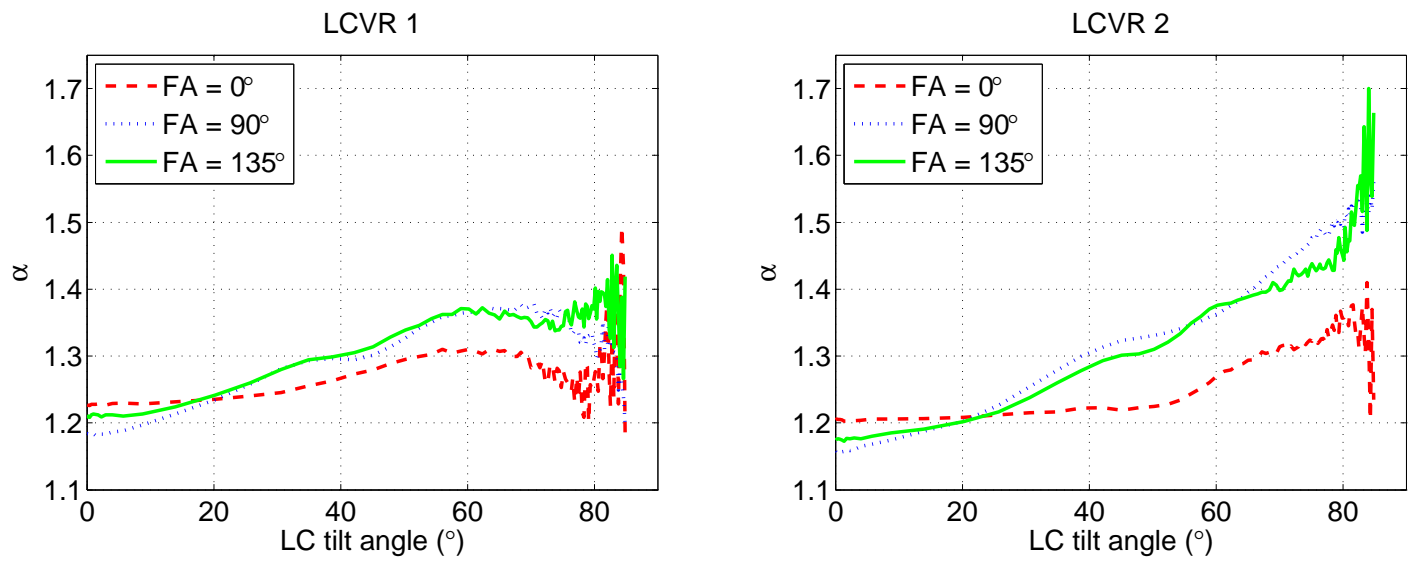

Figure 5.10: The power exponent $\alpha$ as a function of tilt angle for the two LCVRs at the three fast axis orientations 


\section{Discussion}

This work uses a fractal-Born model and spectral scatter effects for characterizing the structural organization of nanometer-sized liquid crystals in an LCVR. The size of the liquid crystals suggest that the scatter is in the Rayleigh regime, where scatter exhibits a power law dependence of $\lambda^{-4}$. However, the results given here show otherwise, as the data exhibit a power law dependence of $\lambda^{-3.4}-\lambda^{-2.4}$. This is clearly non-Rayleigh behavior, and these results show that there is an additional scatter effect present besides typical Rayleigh scattering. The effects seen here are organizational effects, due to the high density and anisotropic structural organization of the LCs. If the LC density were that of a dilute suspension, we would see Rayleigh scattering.

Nematic liquid crystals exhibit an anisotropic structural organization. This anisotropy originates from features of the nematic mixture, as the rod-like LCs have different dielectric constants along their long and short axes, and the LCs exhibit longrange orientational order $[108,110]$. Although the model adopted here (cf. equation (5.2)) assumes isotropy, it is possible to probe LC anisotropy by positioning the LCVR at various fast axis orientations and illuminating the devices with a horizontally polarized electric field. LCVRs are designed such that the long axis of the LCs are orthogonal to the device fast axis, and the plane in which the LCs tilt is parallel to the LC long axis. Thus, changing the relative angle between the incident electric field and the fast axis probes different features of the LC structural organization. 
LC organizational anisotropy is clearly evident in Fig. 5.9, as the figure shows tilt angle-dependent correlation lengths for each of the fast axis orientations. These correlation lengths correspond to the distances within which the LCs exhibit correlated behavior. For example, at a fast axis of $0^{\circ}$, the $\mathrm{LC}$ long axis is perpendicular to the electric field. Therefore, the electric field probes a plane along the short axis of the LCs. On the other hand, for a fast axis of $90^{\circ}$, the LC long axis is parallel to the electric field. In this configuration, the field probes a plane parallel to the long axis of the LCs. A fast axis of $135^{\circ}$ gives correlation lengths higher than the correlation lengths for a fast axis of $0^{\circ}$, but lower than the correlation lengths for a fast axis of $90^{\circ}$. This is because the field explores a plane that is at a $45^{\circ}$ angle relative to the LC orientation, and the field sees behavior that is parallel to both the short and long axes of the LCs.

The fractional scatter (Fig. 5.6) and correlation lengths (Fig. 5.9) also exhibit behavior that is not typically seen with correlated scatter. A decrease in correlation length should produce an increase in scatter, which is not what is observed. The effect seen with the LCVRs is most likely due to the interaction of the LCs with an alignment force. The alignment layer imposes a force on the liquid crystals, which keeps the LCs parallel to the glass windows under the condition of zero applied voltage, and also helps define the fast axis orientation. The LCs must overcome the alignment force in order to rotate. The point at which the LCs overcome this force and transition from an undeformed conformation to a deformed conformation is called 
the Freedericksz transition [108]. As the voltage increases and the LCs approach the Freedericksz transition, they become slightly disorganized. It is hypothesized that this disorganization is responsible for the increase in scatter seen in Fig. 5.6. After the LCs overcome the alignment force, a further increase in voltage exerts a stronger force on the LCs, causing them to experience a more uniform spatial orientation. This increased uniformity in orientation is reflected as the peak in the correlation lengths in Fig. 5.9 for fast axes of $90^{\circ}$ and $135^{\circ}$. For the case of the fast axis at $0^{\circ}$, the electric field does not probe the effects of the Freedericksz transition. This is because the electric field sees only the short axis of the LCs, which is the axis that does not experience the spatial fluctuations in orientation. Another pattern seen in Fig. 5.9 is the decrease in correlation lengths for tilt angles higher than $\approx 40^{\circ}$ for fast axes of $90^{\circ}$ and $135^{\circ}$. Geometrical effects most likely cause this, as the separation distance between LCs changes as they rotate. This can be thought of as a Venetian blind effect, where closing the blinds causes the separation between the slats to decrease. Furthermore, at high drive voltages, $L$ is expected to converge on a common value regardless of fast axis orientation. At high voltages, LCs are approximately parallel to the propagation direction, and their orientation is the same relative to the electric field for all three fast axis orientations.

An additional effect seen in the correlation lengths and exponents is the noise at larger LC tilt angles. These parameters are noisy at larger LC tilt angles because the values of $F_{R}(V, \lambda)-F_{R}(10, \lambda)$ approach the noise floor at the higher drive voltages. 
There also exists an approximate tilt angle at which all three fast axis orientations exhibit similar $L$ values. For LCVR 1 , this happens at a tilt angle of $\approx 10^{\circ}$, and for LCVR 2 at $\approx 8^{\circ}$. These tilt angles are where the LCs overcome the alignment layer forces at the Freedericksz transition.

Another metric for describing LC structural organization is the fractal dimension $D$, which can be estimated from the power exponent $\alpha$. Fractals exhibit similar patterns at all length scales, and can be described as either self-similar or self-affine [133]. If a system is self-similar, then it has uniform scaling in all Euclidean space of dimension $D_{E}$, and the system exhibits isotropy. On the other hand, for a self-affine system, there are different scaling factors for each of the Euclidean coordinates $E$. Thus, self-affine systems can account for anisotropy, and they do so with the Hurst parameter $H$. It has been shown that the fractal dimension $D$ for self-affine systems is $D=D_{E}+1-H$, where $H$ has values on the interval $0<H<1$ [133]. Using equation (5.5), the fractal dimension can also be written as $D=1.5 D_{E}+1-\alpha$.

The Euclidean dimension $D_{E}$ can be interpreted in several different ways. For example, $D_{E}=1$ corresponds to a $1 \mathrm{D}$ signal as a function of time (i.e., music), $D_{E}=2$ corresponds to a surface, and $D_{E}=3$ corresponds to a volume (i.e., a temperature distribution in the atmosphere) [133]. In terms of a system of scatterers, $D_{E}=1,2$ or 3 corresponds to filamentous, sheetlike, or bulk scatterers [131]. 
The fractal dimension $D$ for a self-affine system is linearly related to the exponent $\alpha$ by $D=1.5 D_{E}+1-\alpha$, so the behavior in $\alpha$ reflects the behavior in $D$. Because of this relationship, the discussion focuses on $\alpha$. Equation (5.5) expresses $\alpha$ in terms of $D_{E}$ and $H$. With $D_{E}$ and $H$ exhibiting predefined values, $\alpha$ has distinct intervals that correspond to each Euclidean dimension $D_{E}$. When $D_{E}=1$, the interval for $\alpha$ is $0.5<\alpha_{1}<1.5$, for $D_{E}=2$ the interval is $1<\alpha_{2}<2$, and for for $D_{E}=3$ the interval is $1.5<\alpha_{3}<2.5$. In reference to Fig. $5.10, \alpha$ exhibits values from $\approx 1.1$ at lower LC tilt angles to $\approx 1.5$ at higher tilt angles. Thus, according to the intervals for $\alpha$, the LCs behave like a combination of filamentous and/or sheetlike scatterers. This makes sense on physical grounds, as the LC system consists of rod-like particles with a consistent orientation $[108,110]$. On the other hand, Fig. 5.10 shows that in general, $\alpha$ does not have values higher than 1.5. Values of $\alpha>1.5$ correspond to bulk scatterers, which do not exist in the LC system. The LCs have a specific organization, whereas bulk scatterers would have no organization.

As a final point, when comparing the results from the two LCVRs, the data in Figs. 5.6, 5.9, and 5.10 are qualitatively similar, as they all display similar trends. The differences between the two data sets could be because of the fabrication process for each LCVR. For example, Fig. 5.2 shows that LCVR 2 has a higher overall retardance than LCVR 1. This could mean that the cavity that houses the liquid crystals is thicker for LCVR 2, or the LC mixture for LCVR 2 has a higher refractive index mismatch between LCs and background medium, or both. Additionally, the 
potential fabrication discrepancies between the LCVRs could also explain why the fractional scattering component is higher for LCVR 2.

\section{Conclusions}

This work characterized the structural organization of liquid crystals inside an LCVR by estimating voltage-dependent correlation lengths. These parameters we estimated by using a fractal-Born model and developing a methodology for measuring and isolating LCVR spectral scatter. The results shown here provide insight into two applications, the optimization of LCVR-based polarimetric systems, and the interpretation of structural organization measurements.

Optimization of LCVR-based polarimetric systems requires knowledge of the error sources associated with LCVRs. In Section 3.4.2, LCVR alignment and temperature effects were studied and their impact on system performance was discussed. The work herein focuses on LCVR scatter effects and implications. Measuring LCVR scatter helps establish performance characteristics for a polarimetric system, but performance analysis begins at the device level. For example, during device calibration, the raw tuning curve data oscillates between a minimum and maximum value, with a relative extremum every $1 / 2$ wave. Obtaining the actual tuning curve requires the

application of a phase unwrapping algorithm to the raw data [115]. The unwrapping points are susceptible to noise, such as that created by scattering. Thus, LC scattering can lead to uncertainty at these unwrapping points. 
In addition, the LCs exhibit dispersion effects, which requires the LCVRs to be calibrated at the wavelengths at which they will be used. Moreover, at shorter wavelengths, the LCs exhibit more scattering, and the tuning curves for the LCVRs have more unwrapping points. The increase in both scattering and unwrapping points causes more uncertainty in the tuning curve at lower drive voltages for shorter wavelengths. Therefore, when employing LCVRs in a polarimetric system, knowledge of the wavelength- and voltage-dependent scatter can reveal the most reliable regions of the tuning curve, and impacts system design. In other words, if an LCVR retardance of $2 \pi$ radians is desired (i.e., a low voltage where the scatter is higher), it might be advantageous to use the LCVR at 0 radians (i.e., at high voltage where the scatter is lower). For an LCVR-based Stokes generator, this will impact the method in which the training set Stokes vectors is selected. Similarly, for polarimeters employing LCVRs, this will impact how polarimeter measurements are selected. Additionally, the results shown here can aid in determining the amount of LCVR scatter seen by the detector in a polarimetric system, which also affects performance. These studies provide a thorough understanding of LCVR scatter, alignment, and temperature effects, and how these effects impact polarimetric system performance.

The work presented here also serves as a model for interpreting structural organization measurements. In general, the aim of this dissertation is to infer structural organization parameters from measurements on a given sample, and the work in the current section acts as a model for this. LCVRs are an effective test case because 
there is a priori knowledge about the organization of the LCs. Moreover, the user has some degree of control over the organization through the drive voltage. The effects caused by changing the drive voltage can be observed with a physical justification for the observation. Consequently, these devices are critical tools for validating the process for making structural organization inferences.

\subsection{Terrestrial earth rock}

Assessing the structural organization of the terrestrial earth rock consists of several steps. These steps include imaging various rock regions in transmission mode with the polarimeter, parameterizing the data with a model fit, and then ultimately comparing the parameterization with scanning electron microscopy energy-dispersive spectroscopy (SEM-EDS) measurements. An SEM-EDS system provides quantitative elemental analysis, which can be used for mineral identification [134].

SEMs irradiate a sample with a focused beam of electrons, and then measure the scatter effects from the sample. The scattering can be inelastic, which ejects electrons from atoms within the sample. The ejected electrons are called secondary

electrons, and the ejection leaves a vacant spot that causes instabilities in the atom. To regain stability, higher-energy outer shell electrons fill the vacancy, but release excess energy in the form of x-rays. SEM-EDS systems measure the quantity and energy of these x-rays. Peaks at specific locations in the x-ray spectrum correspond to 


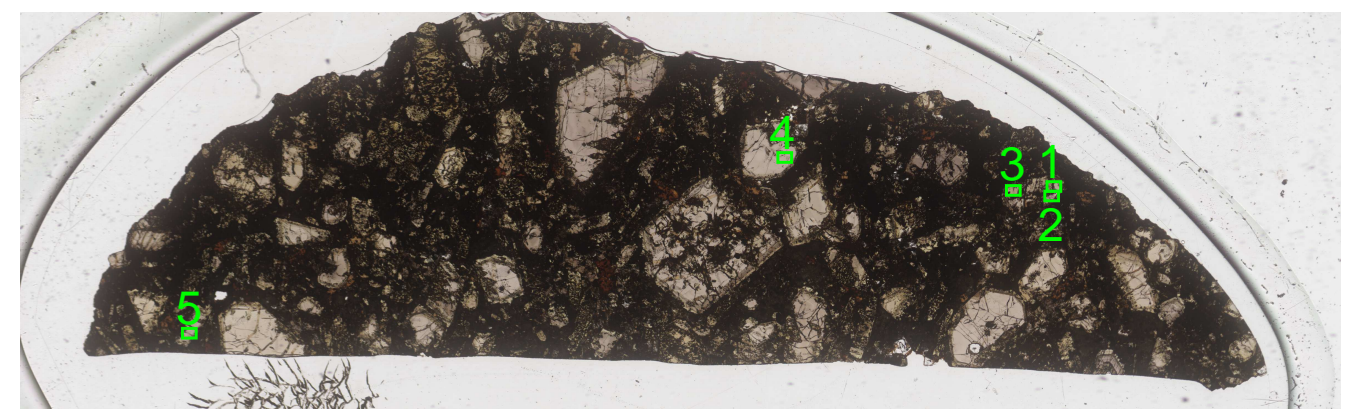

Figure 5.11: Brightfield microscope image of the terrestrial earth rock. This image did not come with a scale bar, but the sample is $\approx 8 \mathrm{~mm}$ wide, $\approx 28 \mathrm{~mm}$ long, and has a thickness of $\approx 36 \mu \mathrm{m}$. The green numbered boxes correspond to regions imaged by the polarimeter.

particular elements. Thus, the sample atomic structure dictates the shape of the EDS spectrum, providing a means for elemental analysis and mineral identification [134].

Figure 5.11 shows a brightfield microscope image of the terrestrial earth rock sample, which came from the Cascadia Meteorite Laboratory at Portland State University. The sample image did not come with a scale bar, but the sample is $\approx 8 \mathrm{~mm}$ wide, $\approx 28 \mathrm{~mm}$ long, and has a thickness of $\approx 36 \mu \mathrm{m}$. Shown in this figure is an overlay of several green numbered boxes, which represent regions that were imaged by the polarimeter. The sample thickness was estimated using the SEM-EDS results, separate crossed-polarizer microscope measurements, and the Michel-Levy birefringence chart. The SEM-EDS results were used for identifying the minerals, from which a birefringence was estimated. Crossed-polarizer microscope measurements produced images with interference colors, which were visually inspected and compared to the Michel-Levy chart. This chart relates interference colors to thickness 
and birefringence, and was used for estimating the sample thickness.

The sample was modeled as a birefringent waveplate, which has a Mueller matrix that is parameterized on a retardance $\delta$ and fast axis $\theta$ (see Appendix B for the complete Mueller matrix of a birefringent waveplate). For the model fitting routine, the first step involves selecting the most reliable Mueller matrix element from the measurement. Elements $(3,3)$ and $(4,3)$ were selected as they have a low sensitivity to alignment and temperature effects (see Section 3.4.2), and they produced accurate results during the polarizer and retarder test measurements (see Section 3.4.4). Element (4,3) was used for the first four regions, while element $(3,3)$ was used for region 5. For region 5, element $(3,3)$ showed less error in the model fits than element $(4,3)$; thus it was used for that particular region. Next, the analytic form of the given element of the retarder Mueller matrix was fit to the spectral data for the same element, at each point in the element image. The form of the models are

$$
\begin{aligned}
& m_{33}=\sin ^{2}(2 \theta)+\cos \left(\frac{2 \pi t \Delta n_{e f f}}{\lambda \cos \psi_{e f f}}\right) \cos ^{2}(2 \theta) \\
& m_{43}=-\sin \left(\frac{2 \pi t \Delta n_{e f f}}{\lambda \cos \psi_{e f f}}\right) \cos 2 \theta
\end{aligned}
$$

where $t$ is the thickness, $\Delta n_{\text {eff }}$ is the effective birefringence, $\lambda$ is the incident wavelength, $\psi_{\text {eff }}$ is the effective incidence angle, and $\theta$ is the fast axis angle. Values of $t=36 \mu \mathrm{m}$ and $\psi_{\text {eff }}=9.38^{\circ}$ were used, and measurements were made at wavelengths of $\lambda=500-720 \mathrm{~nm}$ at $20 \mathrm{~nm}$ increments. The other two variables $\left(\Delta n_{\text {eff }}\right.$ and $\left.\theta\right)$ 
are the free parameters of the fitting routine.

\section{Results}

Figure 5.12 shows the Mueller matrix images for region 1 at wavelengths of $500 \mathrm{~nm}$ and $720 \mathrm{~nm}$. The Mueller matrix is normalized to the (1,1) element, resulting in this element having a constant value of one. Instead of showing an element with a constant value, the $(1,1)$ elements in Fig. 5.12 show the raw intensity image with a separate colorbar. This illustrates regions with higher SNRs. The remaining elements of the Mueller matrix are still normalized to the $(1,1)$ element, and have values on the interval $[-1,1]$. Figure 5.13 shows model fits to the analytic form of element $(4,3)$ for two individual points from region 1 . In this figure, the model for element $m_{43}$ was fit to the $m_{43}$ data, then the fitted parameters were used in the analytic forms for the remaining Mueller matrix elements. One of the points was selected from a region with high SNR, while the other was selected from a region with low SNR.

Figure 5.14 shows the results from applying the fitting routine to region 1 . In the top left corner of this figure is the raw intensity image from the polarimeter, and in the top right corner is the SEM-EDS layered image. The remaining images in the figure are 2D maps of the fit parameters $(\Delta n$ and $\theta)$ and the RMS error. The polarimeter acquired images at a magnification of $\approx 20 \times$, which produced a field of view of $\approx 233 \mu \mathrm{m}$ by $311 \mu \mathrm{m}$. SEM images were also acquired at $\approx 20 \times$ magnification, but due to minor inaccuracies in the SEM location system, the exact polarimeter 


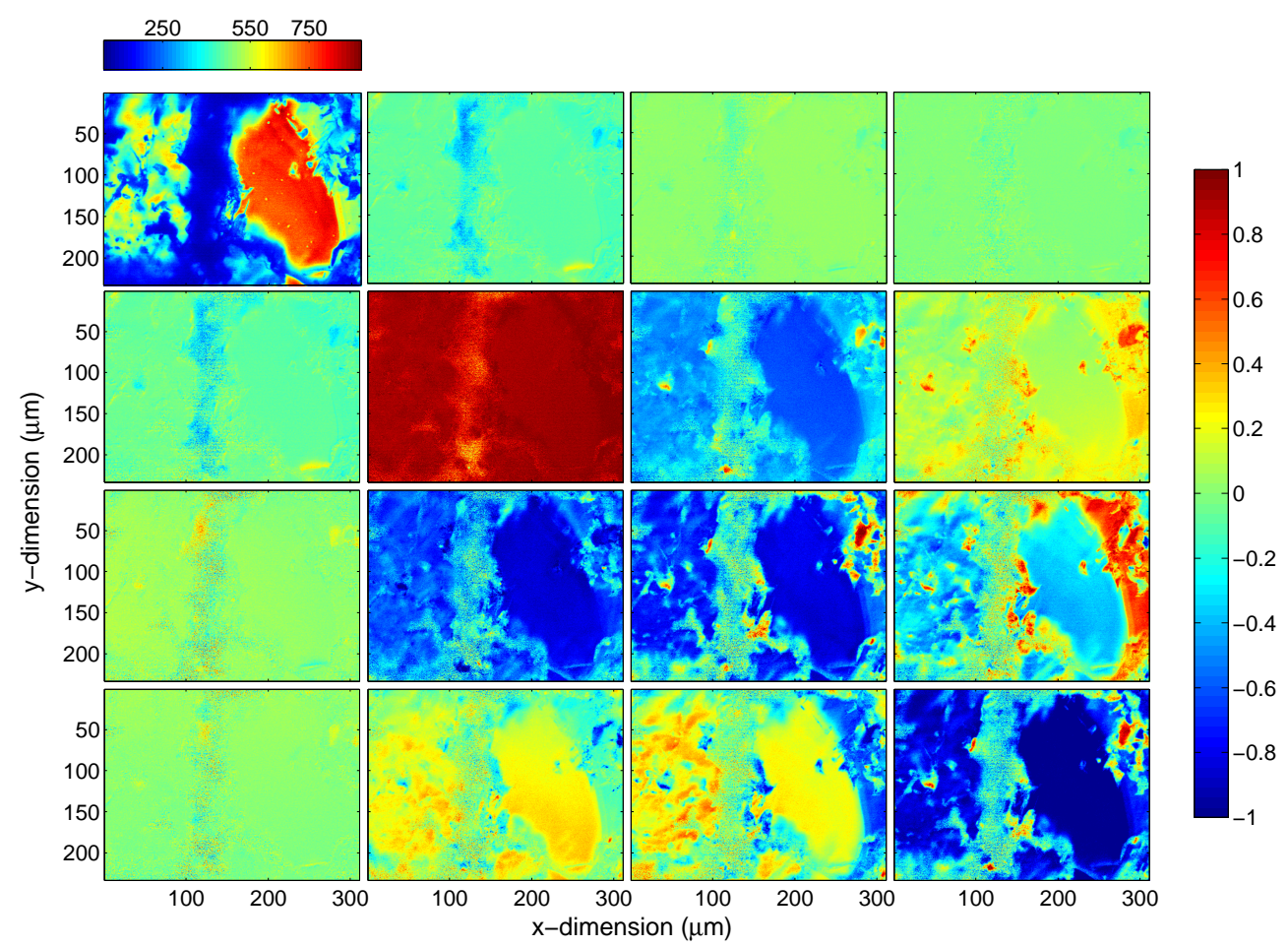

(a) $500 \mathrm{~nm}$

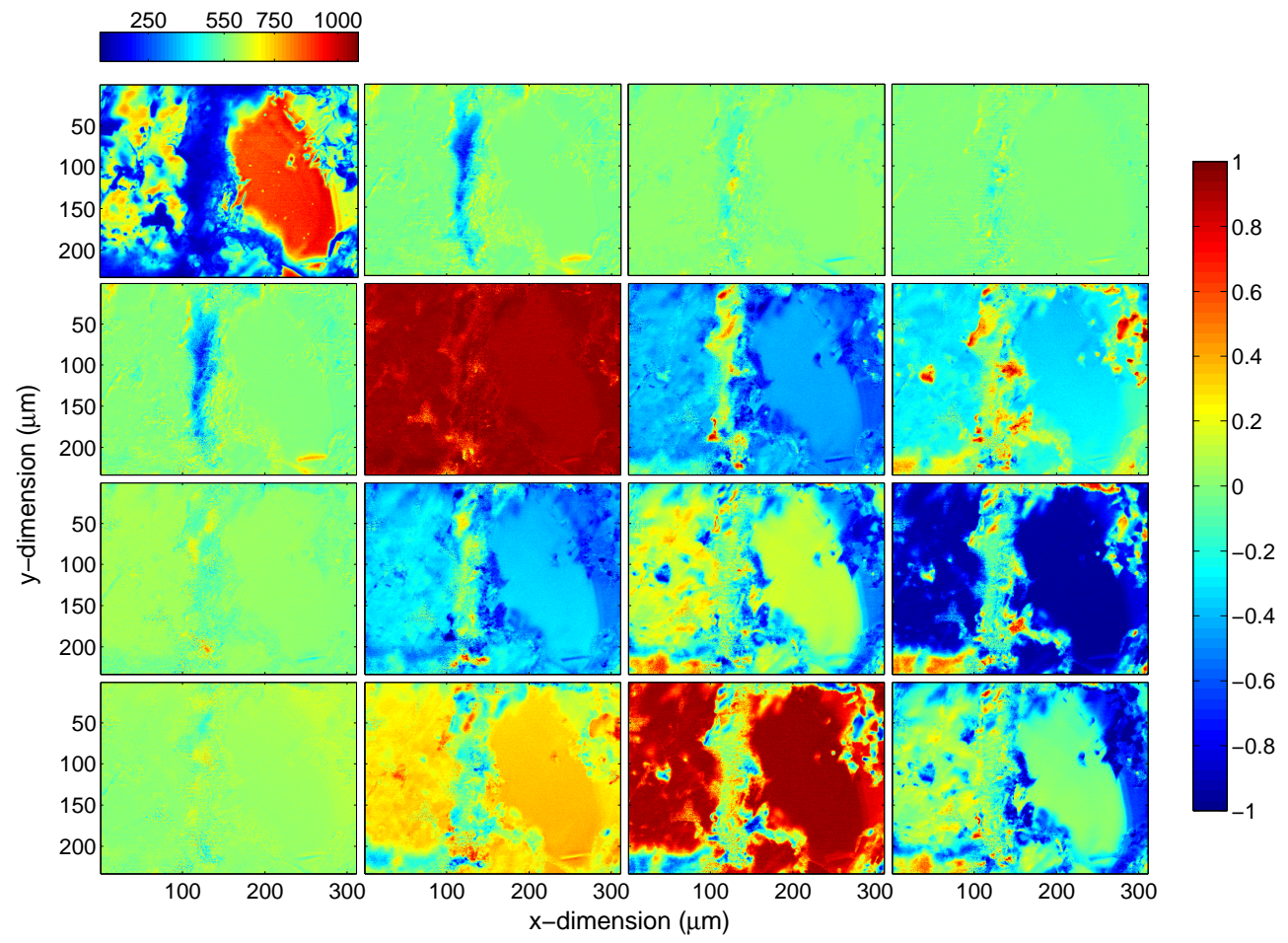

(b) $720 \mathrm{~nm}$

Figure 5.12: Mueller matrix images for region 1 at two wavelengths. The $(1,1)$ element is the raw intensity image with a separate colorbar. 


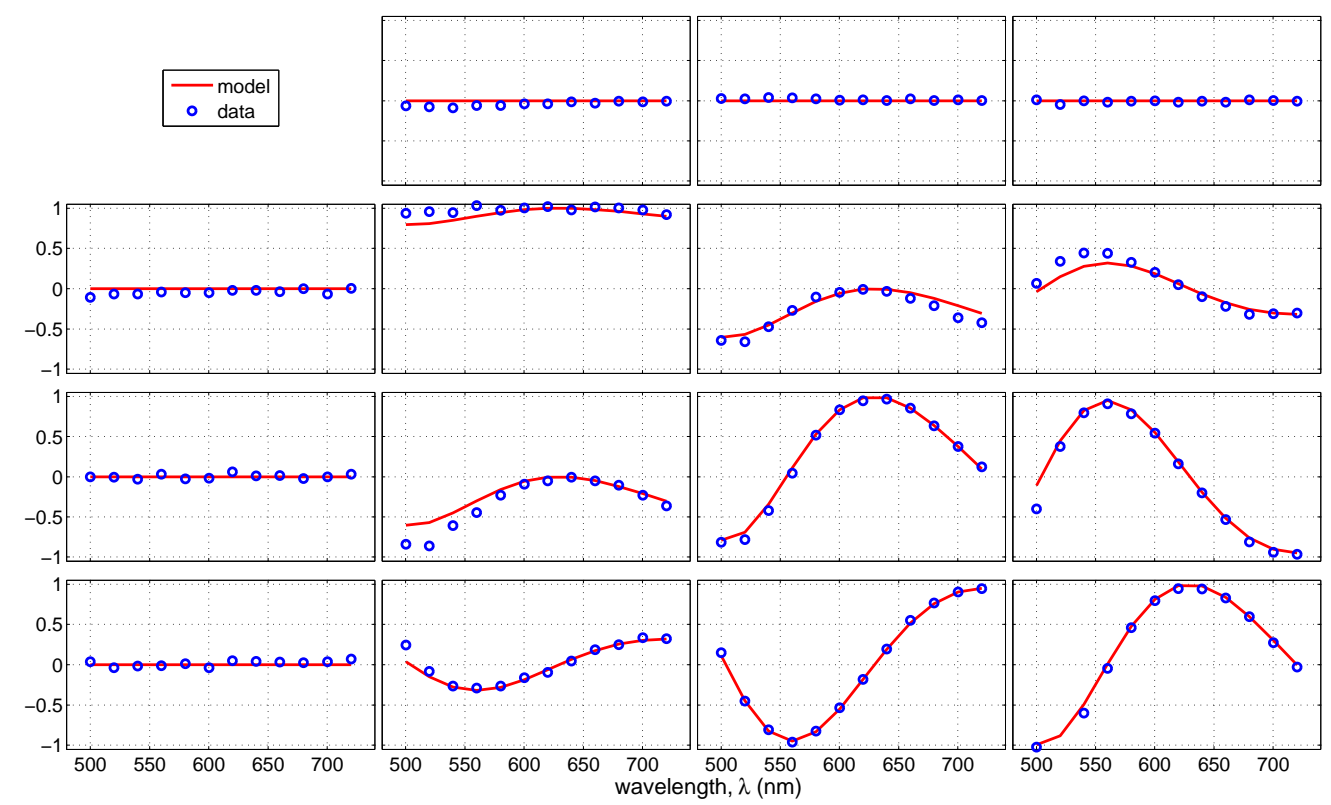

(a) High SNR region

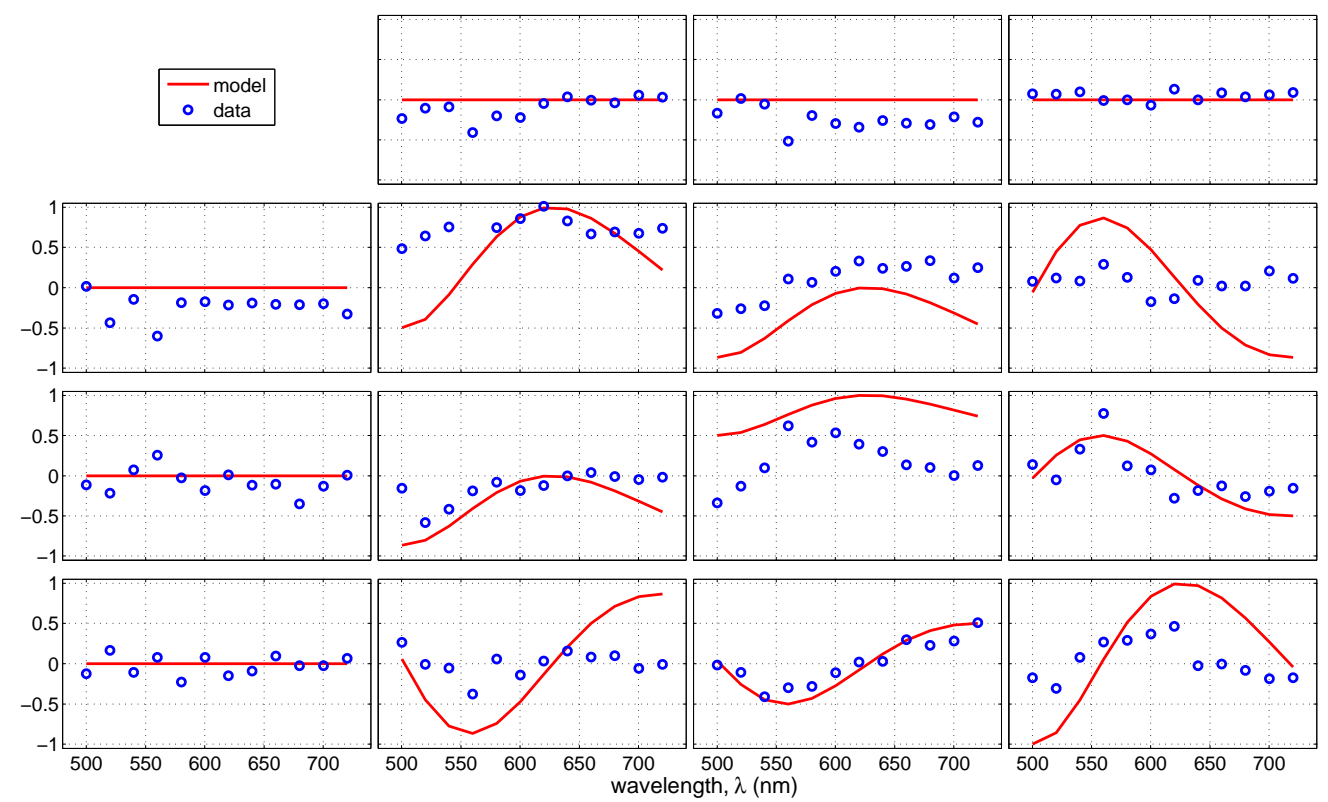

(b) Low SNR region

Figure 5.13: Model fits to the $(4,3)$ element for a single point from region 1 . The fitted parameters were then applied to the analytic forms of the remaining Mueller matrix elements. 

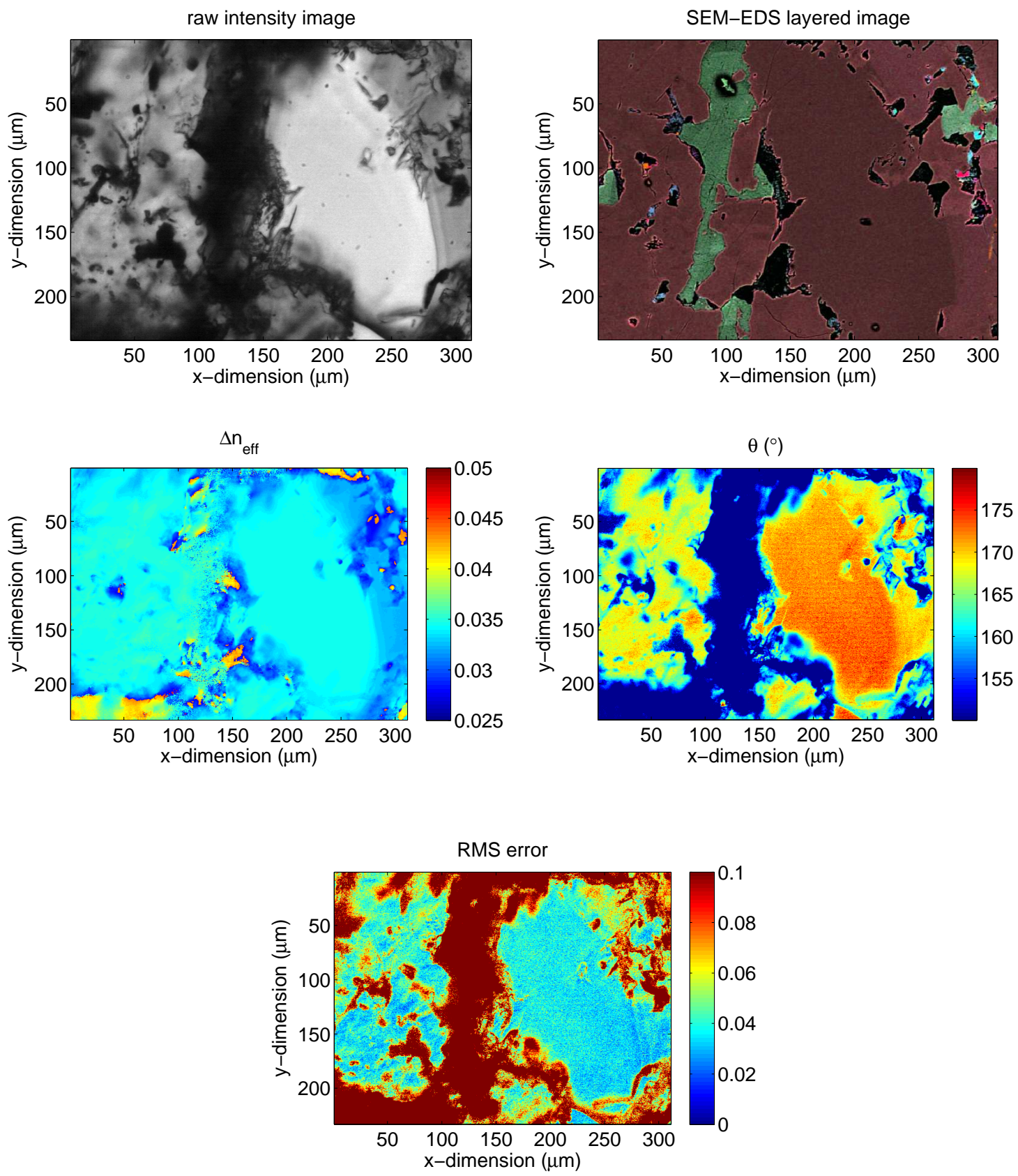

Figure 5.14: Results from region 1. Raw intensity image (top left) and SEM-EDS image (top right), along with results from the model fits to the Mueller matrix element $(4,3)$ (bottom three images). 

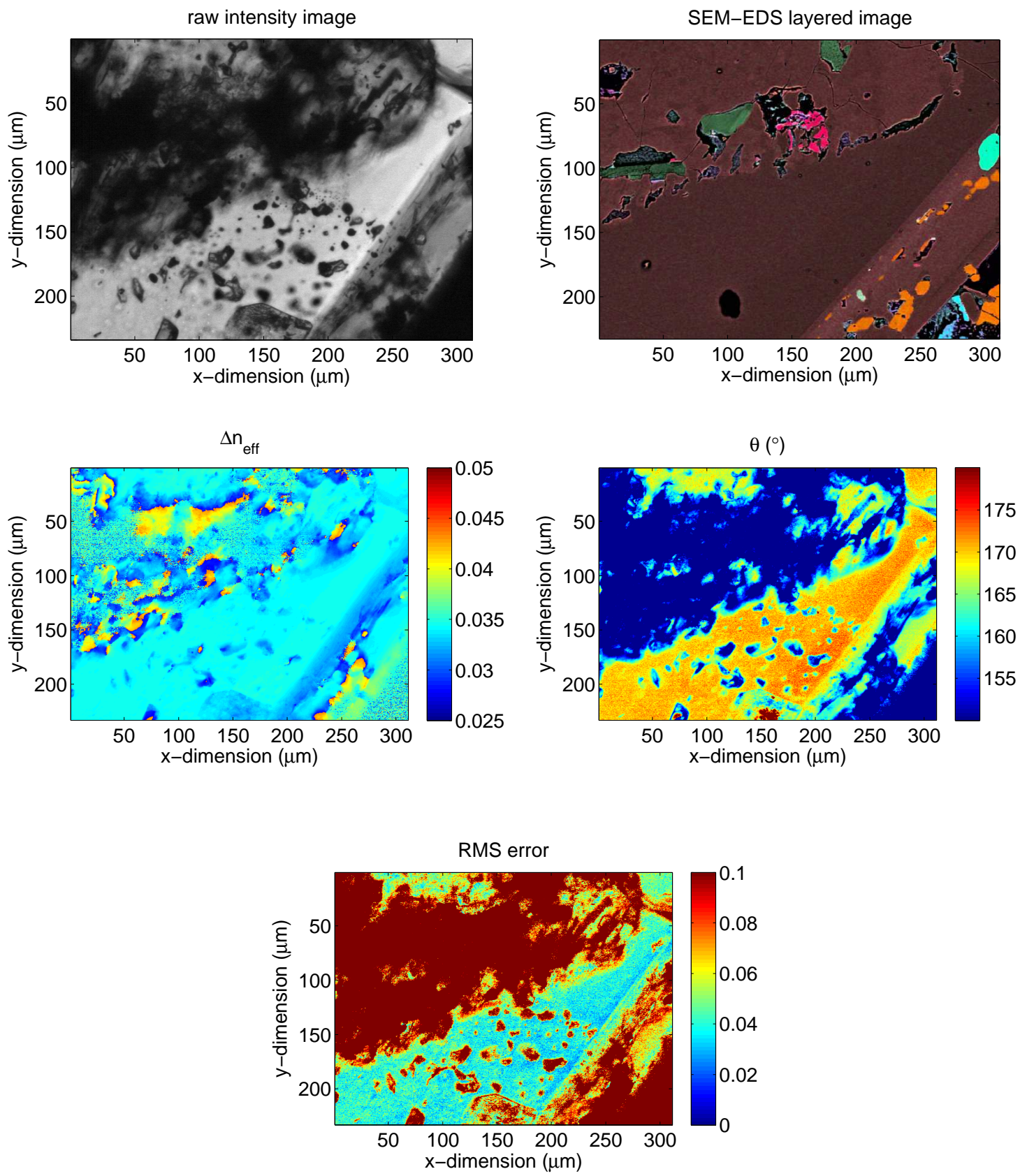

Figure 5.15: Results from region 2. Raw intensity image (top left) and SEM-EDS image (top right), along with results from the model fits to the Mueller matrix element $(4,3)$ (bottom three images). 

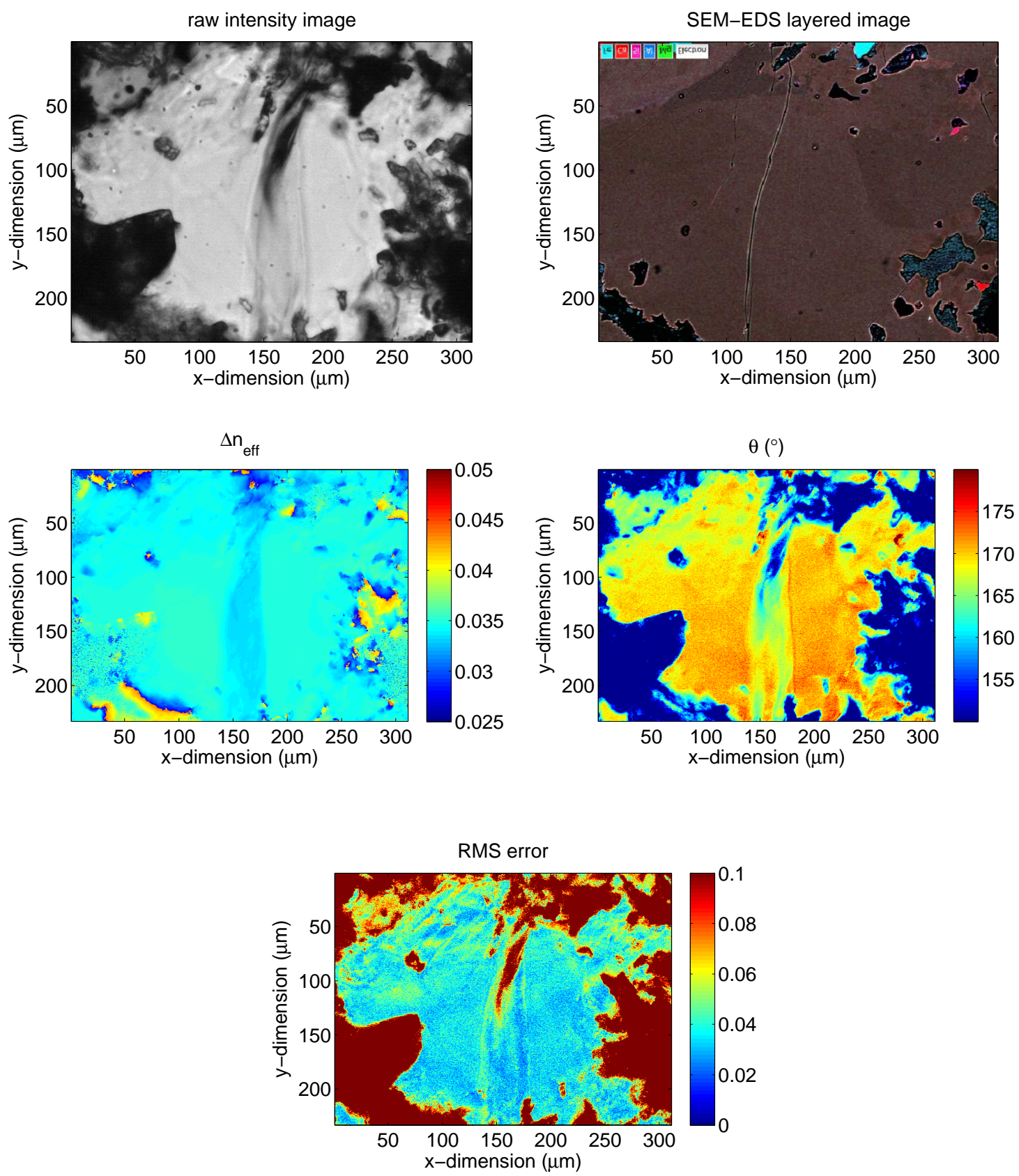

Figure 5.16: Results from region 3. Raw intensity image (top left) and SEM-EDS image (top right), along with results from the model fits to the Mueller matrix element $(4,3)$ (bottom three images). 

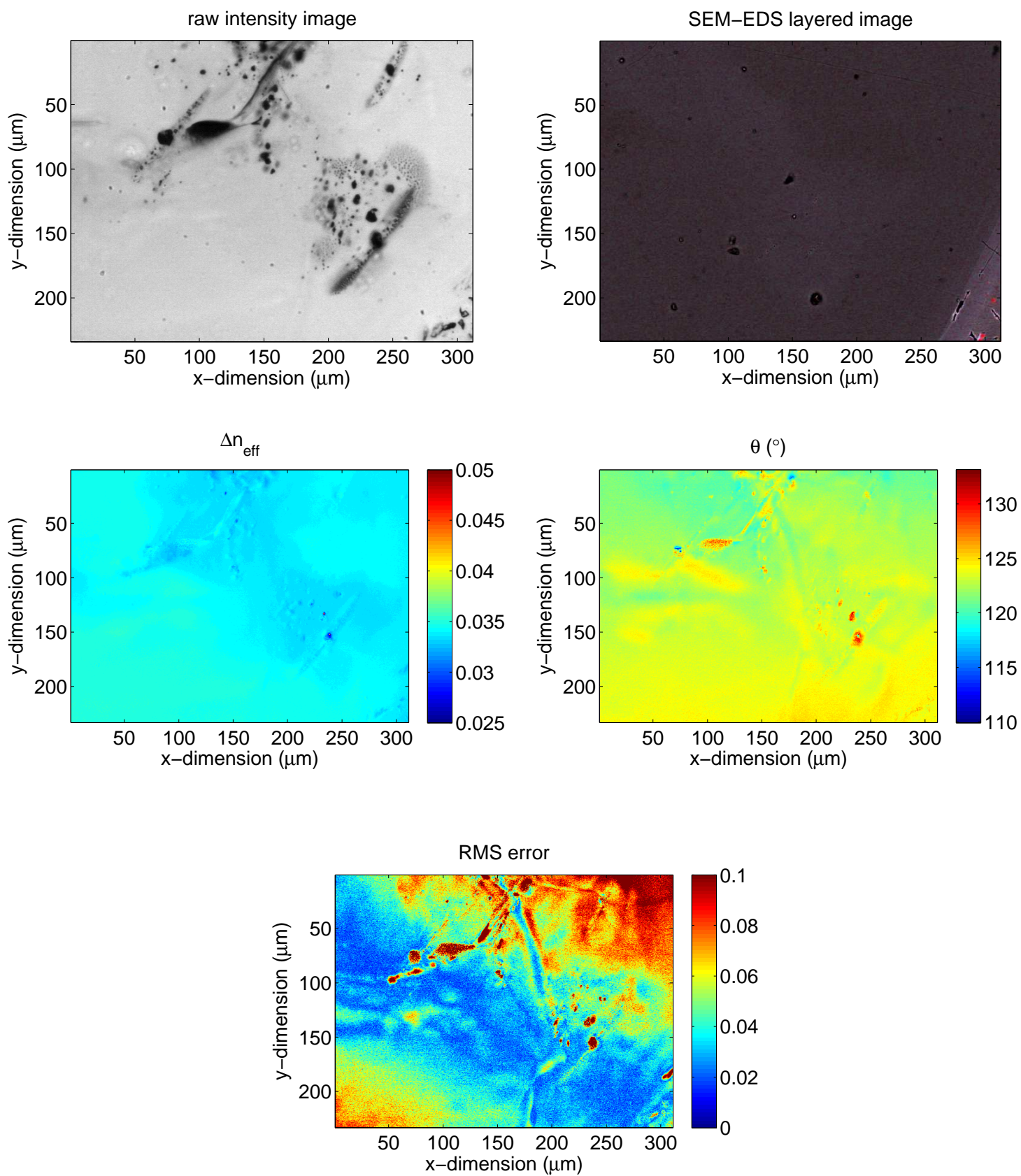

Figure 5.17: Results from region 4. Raw intensity image (top left) and SEM-EDS image (top right), along with results from the model fits to the Mueller matrix element $(4,3)$ (bottom three images). 

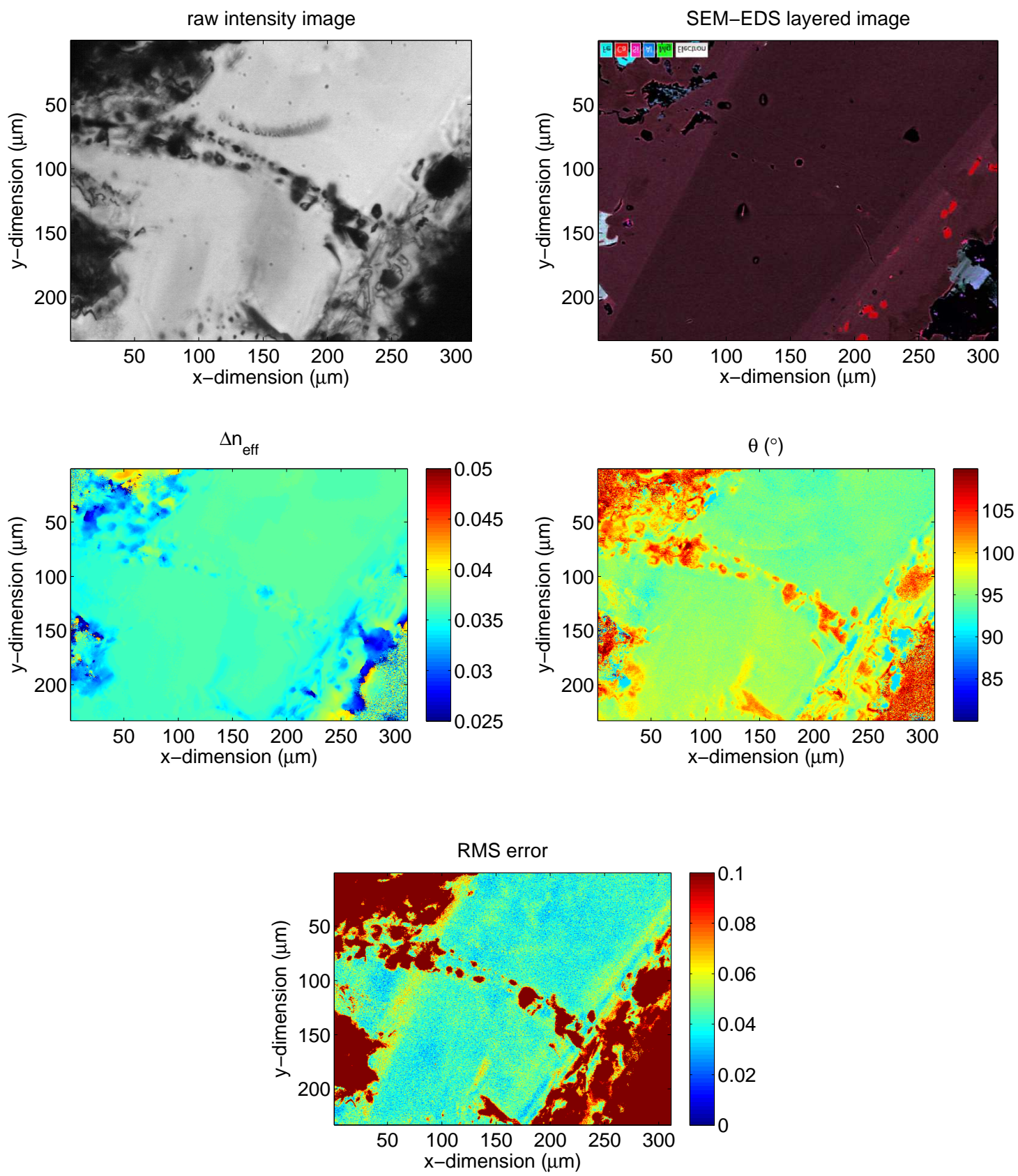

Figure 5.18: Results from region 5. Raw intensity image (top left) and SEM-EDS image (top right), along with results from the model fit to the Mueller matrix element $(3,3)$ (bottom three images). 
regions were not recovered. Thus, the SEM and polarimeter imaged slightly different regions, although main features are easily identifiable between the two. Figures 5.155.18 show the same type of results as Fig. 5.14, but for the remaining four regions. For Figs. 5.14-5.18, the 2D maps for $\Delta n$ and the RMS error are all on the same scale, while the scale for $\theta$ varies according to the plotted values.

\section{Discussion}

The EDS images in Fig. 5.14-5.18 are used for mineral identification, and the remaining images show the ability of the polarimeter to parameterize structural organization. The SEM-EDS layered images provide the atomic values of the various phases (data not shown), from which the minerals can be identified, and their refractive indices estimated. Polarimetric results parameterize structural organization with an effective birefringence and fast axis angle, and from these estimates, can provide insight into mineral structural organization.

Several minerals have been identified in the SEM-EDS images. The reddishbrown mineral phases of all five regions belong to a mineral group called pyroxenes, which are single chain silicates with a fundamental unit of $\mathrm{SiO}_{3}$. Pyroxenes are typically found in igneous or metamorphic rocks, and their compositions vary based on the types of elements present [135]. One main type of pyroxene, augite, has been identified in the five regions. Augite can have varying amounts of calcium, magnesium, and iron, where the particular elemental distribution influences the refractive index. 
From the atomic values in the EDS maps, the primary phases have been identified as augite in all five regions (Figs. 5.14-5.18), but with a wide compositional spread. All of the other phases (represented by the other colors in the SEM-EDS images) exhibit either weak birefringence, isotropy, or opacity. For example, the green phase in regions 1 and 2 is most likely an isotropic glass. In region 2, the pink phase is sphene, which has high refractive indices $(n=1.84-2.11)$ and is often opaque, especially in the form of small crystals $[136,137]$. The orange phase in region 2 is a fluorapatite, which has very weak birefringence, $\Delta n=0.003-0.005$ [138]. The bright green phase in region 2 is a type of spinel (an isotropic mineral), and the red phase in region 5 is another isotropic mineral, perovskite.

Augite is a biaxial crystal, thus it has two optical axes and three principal indices of refraction. The orientation of the crystal structure within the mineral is unknown, and this results in ambiguity with respect to the orientation of the three primary refractive indices. This ambiguity (along with the elemental distribution) gives rise to a range of birefringence values, as augite has values of $\Delta n=0.025-$ 0.039, $[135,136]$. The birefringence maps for Figs. 5.14-5.18 show values within the acceptable range for augite. Changes within the birefringence maps could be an indicator of variations in chemical composition. For example, the birefringence maps in Figs 5.14 and 5.15 show sharp birefringence transitions from light blue $(\Delta n \approx$ $0.0345)$ to blue ( $\Delta n \approx 0.0325)$ along the edge of these two figures. This could reflect slight changes in the amount of calcium, magnesium, or iron in the augite. 
The fast axis maps represent the orientation of the birefringence within the plane of the sample. Although the fast axis does not correspond to a specific mineral, it still reveals mineral information. For example, the EDS image for region 3 (Fig. 5.16) shows a vertical black line in the center. This could be a deformation in the mineral, which would produce strain in the crystal structure. A strain could cause the area near the deformation to experience a different optical orientation, to which the fast axis parameter would be sensitive. Thus, the change in fast axis orientation in the middle of Fig. 5.16 could be a result of deformation. Additionally, fast axis variations could also reflect changes in chemical composition, as the addition or subtraction of particular elements could introduce subtle changes in the crystal structure orientation.

When comparing the images from the two systems, prominent dark areas are noticeable in the polarimeter images, but not in the EDS images. For example, in all five regions, large dark areas of low SNR are visible in the raw intensity images, but not necessarily the EDS images. This effect results from the functionality for each modality, as the polarimeter operates in transmission, and the SEM operates in reflection. The polarimeter is sensitive to optical attenuation throughout the thickness of the sample. The low SNR areas represent a material that is either highly scattering, highly absorbing, or both. Conversely, the SEM probes only surface effects, as the maximum depth of emission for secondary electrons is $\approx 50 \mathrm{~nm}$ [134]. Although the optical measurements suffer from regions of low SNR, these regions signify changes in structural organization features (i.e., non-transparent materials or inhomogeneities) 
that are not necessarily limited to the surface. Additionally, the low SNR areas in the raw intensity images correspond to areas of high error in the RMS error maps.

Finally, the values for thickness and illumination incidence angle impact the estimated birefringence values. An increase in either thickness or incidence angle will decrease the estimate of effective birefringence. The incidence angle is an effective angle that represents the cone of illumination, where the cone has a maximum illumination angle of $16.26^{\circ}$.

\section{Conclusions}

The work herein characterized the structural organization of minerals using a birefringent waveplate model. This characterization was in terms of the effective birefringence and fast axis angle. The effective birefringence quantifies the anisotropy along perpendicular axes, and is sensitive to refractive index variations. These variations could be brought about by changes in chemical composition, or subsurface inhomogeneities (e.g., isotropic glass or opaque minerals) that disrupt the crystal structure. The fast axis describes the orientation of the anisotropy relative to a horizontal axis. Fast axis variations could be indicative of deformations in crystal structure (e.g., cracks). In conclusion, these results parameterized structural organization in terms of optical anisotropies, which then can be related to physical features within the mineral. 


\subsection{Butterfly wing scales}

Polarimeter backscatter imaging measurements were made on butterfly wing scales. The scales were produced by Connecticut Valley Biological Supply Company and came mounted on a glass slide. The structural organization from this sample could not be assessed due to limitations of the imaging system in the backscatter configuration. This section shows the results and describes the limitations.

\section{Results}

Figure 5.19a shows the raw intensity backscatter image of the butterfly wing scales. The wing scale slide was oriented in the imaging plane such that the polarimeter collected the $30^{\circ}$ scatter, similar to the calibration process with the mirror (see Section 3.4.4). However, this signal was the $30^{\circ}$ specular reflection from the cover slip, so the wing scale slide was slightly rotated in the sample plane such that the specular reflection was not imaged.

Figure 5.20 shows backscatter Mueller matrix images of the wing scales for two wavelengths, $560 \mathrm{~nm}$ and $720 \mathrm{~nm}$. Images acquired at wavelengths below $560 \mathrm{~nm}$ have low SNRs and are not shown. 


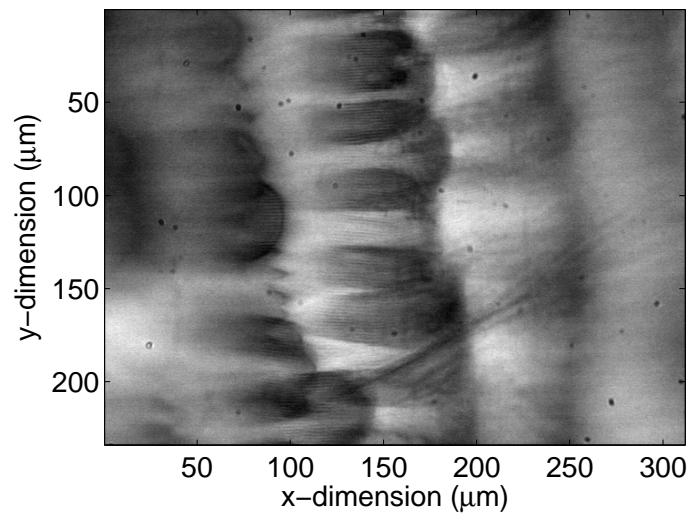

Figure 5.19: Raw intensity backscatter image of the butterfly wing scales.

\section{Discussion}

The raw intensity figures show the effects of the polarimeter limitations. For example, the shallow depth of field $(\approx 3.5 \mu \mathrm{m})$ of the imaging objective results in a narrow region of focus when imaging at oblique angles. In a typical imaging system, the object plane, image plane, and imaging lens are all parallel to each other. If the sample is parallel to these planes, and within the depth of field, it will be clearly imaged at the image plane. However, if the sample is tilted, which is the case here, only the sample region within the depth of field will be focused sharply. Additionally, weakly scattering samples such as the scales do not provide a signal with an adequate SNR. Even with these limitations, the central portion of the image still can be used for extracting quantitative data. However, the spectral polarization signature from the center of the image exhibited little variation with wavelength. Either the sample exhibits minimal spectral polarization effects, or the low SNR spoils the signal. The 


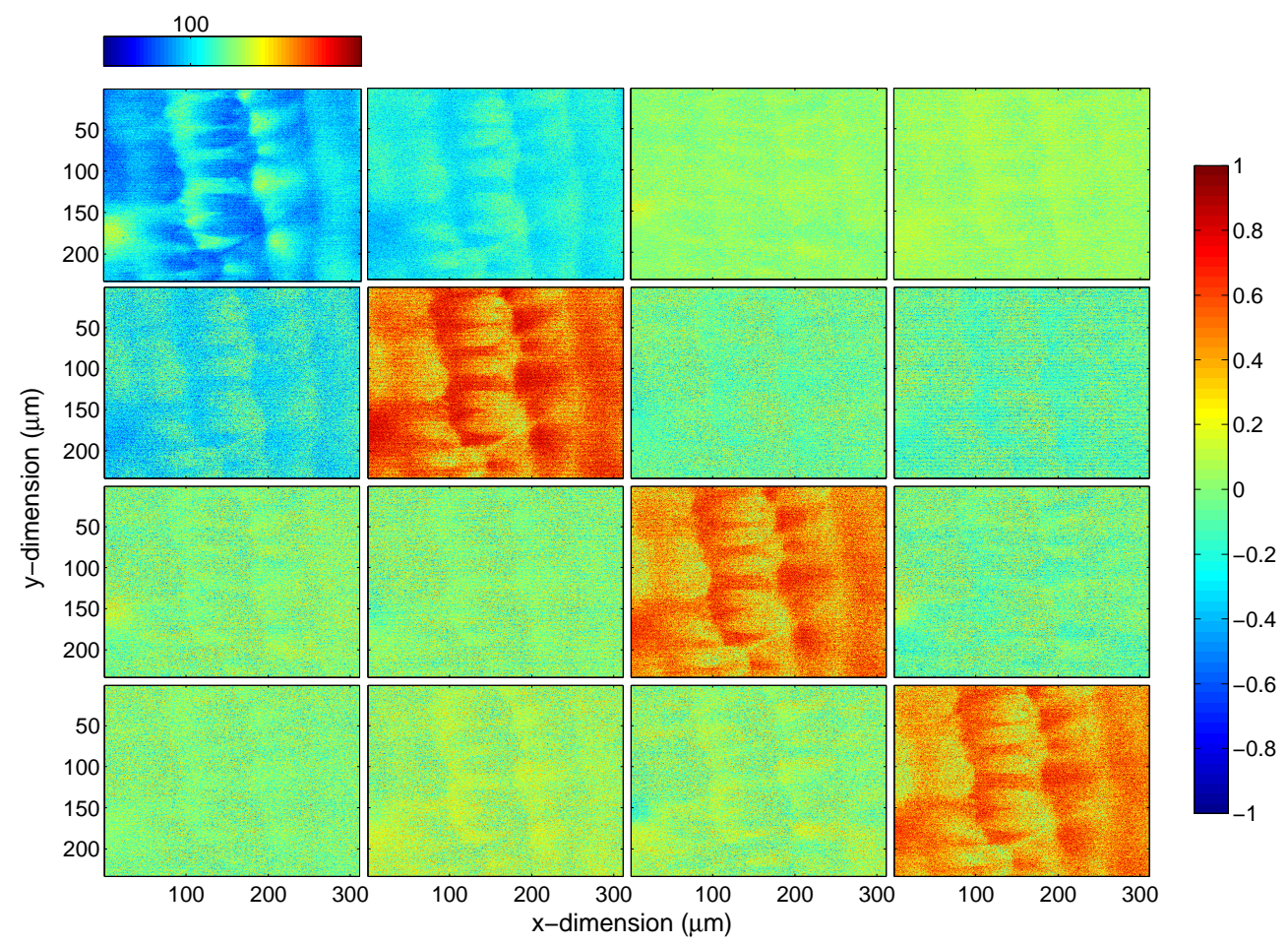

(a) $560 \mathrm{~nm}$

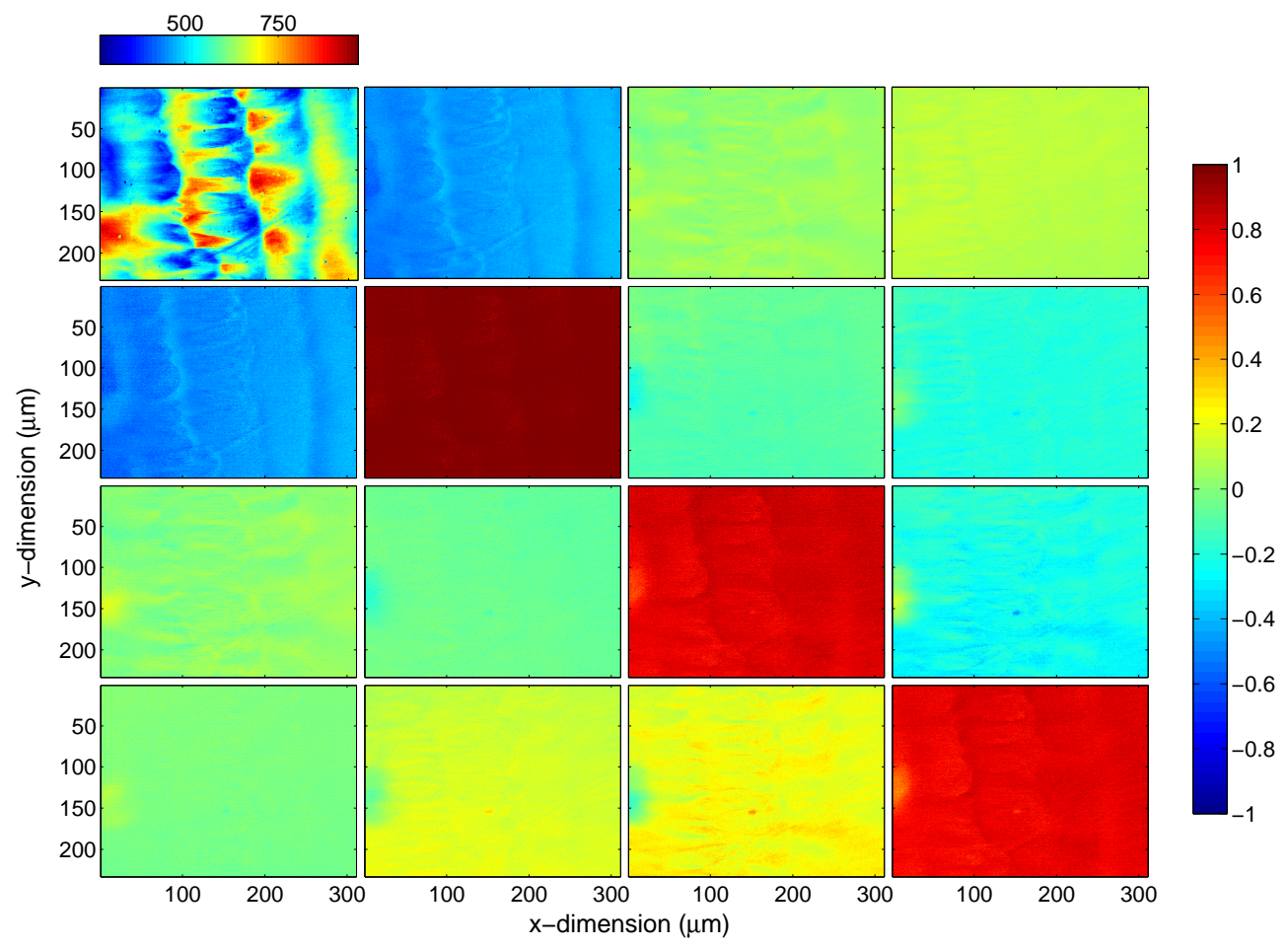

(b) $720 \mathrm{~nm}$

Figure 5.20: Mueller matrix backscatter images from the butterfly wing scales at two wavelengths. The $(1,1)$ element is the raw intensity image with a separate colorbar. 
latter is more likely, as the subwavelength structures have the potential for providing spectral polarization effects.

\section{Conclusions}

The spectral imaging polarimeter is capable of making goniometric measurements, but in a limited capacity. It is possible to make backscatter measurements with this system, however, the resulting images have a limited depth of field. Additionally, low SNRs preclude polarization assessment.

The aforementioned limitations can potentially be solved with a series of upgrades. For example, the blurred image regions could be compensated for with a configuration used in ophthalmic slit-lamp microscopes and view cameras [139]. This configuration, the Scheimpflug architecture, can be used in imaging systems where the image plane, object plane, or imaging lens are not parallel with each other. By introducing a series of tilts to the components, the Scheimpflug architecture rotates the near and far limits of the depth of field so that the sample is within these limits.

SNR could be improved by using a different light source. The current light source illuminates the condenser portion of the measurement system with a $6 \mathrm{~mm}$ diameter fiber. Light exiting the fiber diverges rapidly, and a significant amount of power is lost during the collimation process. Additionally, the LCTF transmits less than $50 \%$ of incident light, as it incorporates polarizers in its construction. Thus, a 
stronger light source with a fiber that produces less-divergent light could increase the SNR of the system.

An additional technique for improving SNR could be implementing a realtime feedback algorithm for controlling camera settings. Because of the linearity of the camera exposure time, a real-time feedback algorithm for controlling exposure time can be implemented. This algorithm would consist of an automated, real-time optimization of the exposure time for each of the six Stokes vector measurements. After image acquisition, the images can be scaled an amount corresponding to the exposure time, thus normalizing the images for subsequent data processing. This technique would utilize the dynamic range of the camera, and ideally would decrease measurement noise.

\subsection{Nanofibers}

Backscatter imaging measurements were also made on a nanofiber mat. FibeRio manufactured the nanofiber mat, which was $\approx 3 \mu \mathrm{m}$ thick and consisted of multiple layers of nanofibers with no preferred orientation. The mean diameter of the nanofibers was $\approx 237 \mathrm{~nm}$. 


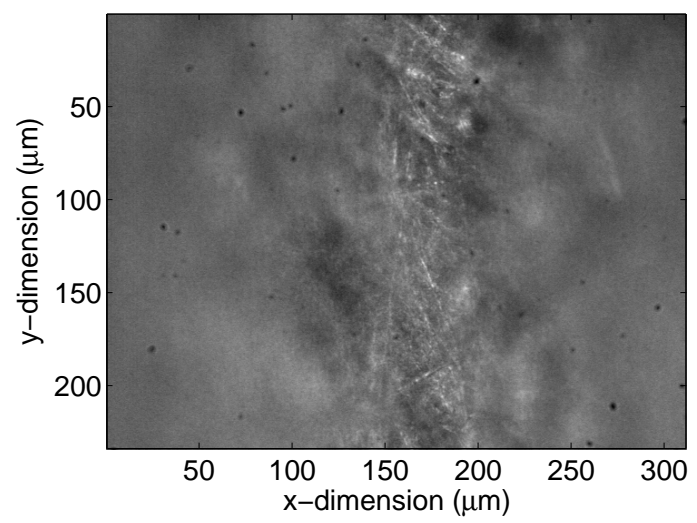

Figure 5.21: Raw intensity backscatter image of the nanofiber mat.

\section{Results}

Figure 5.21 shows the raw intensity backscatter image of the nanofibers. Unlike the butterfly wing scales, no cover slip was used on this sample, thus the slide containing the nanofibers was oriented such that the signal at an angle of $30^{\circ}$ was measured.

Figure 5.22 shows backscatter Mueller matrix images of the nanofibers for two wavelengths, $500 \mathrm{~nm}$ and $720 \mathrm{~nm}$. The backscattered signal from the nanfibers was higher than that of the wing scales, which allowed for measurements at shorter wavelengths.

\section{Discussion}

Similarly to the butterfly wing scale images, the nanofiber backscatter images show the effects of the polarimeter limitations. These effects worsen, however, when the sample consists of a series of point scatterers, which causes a further decrease in the 


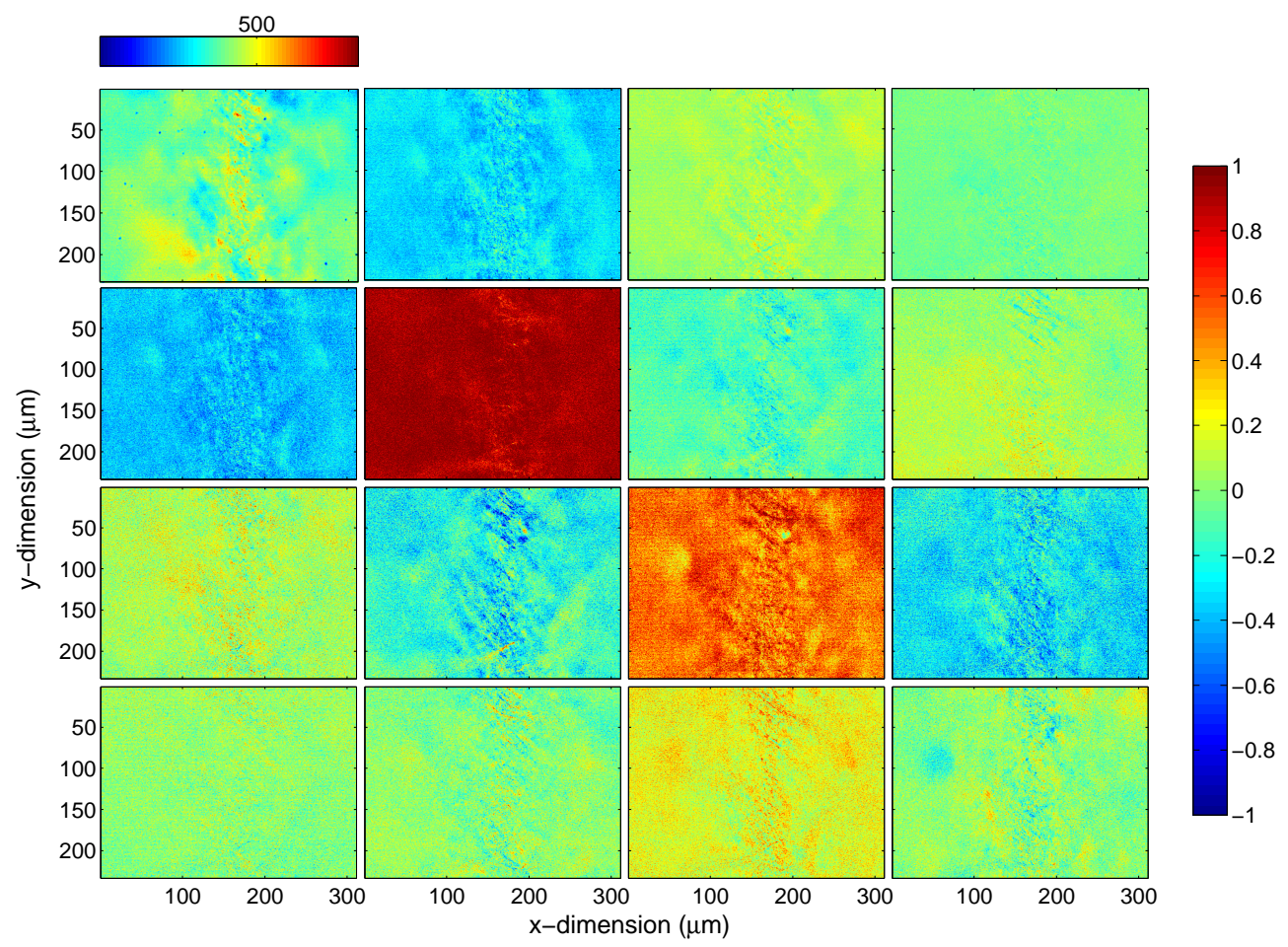

(a) $500 \mathrm{~nm}$

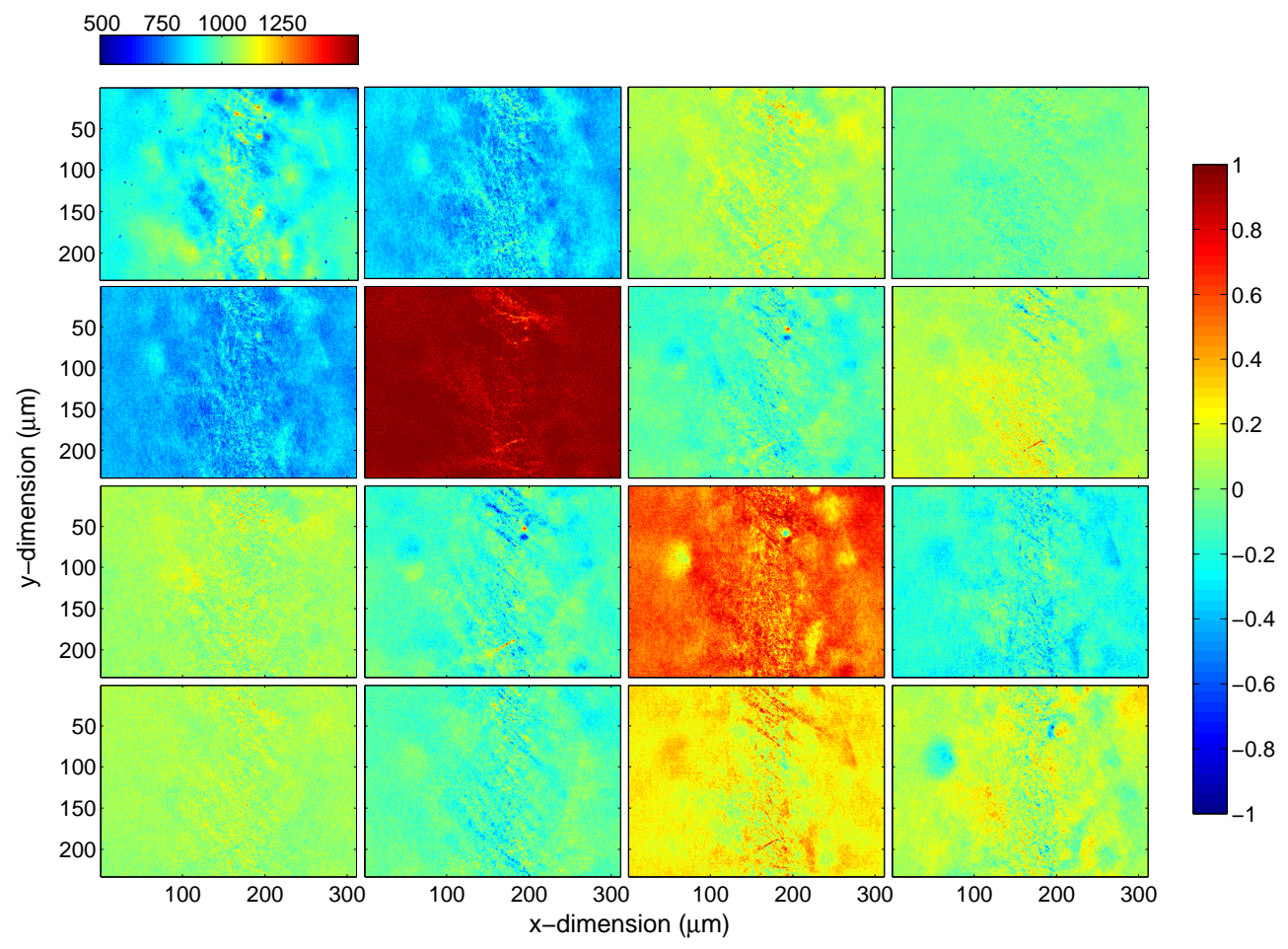

(b) $720 \mathrm{~nm}$

Figure 5.22: Mueller matrix backscatter images from the nanofibers at two wavelengths. The $(1,1)$ element is the raw intensity image with a separate colorbar. 
region of focus. Although the nanofibers produce a higher signal than the wing scales, the nanofiber signal still has a low SNR, precluding polarization assessment.

\section{Conclusions}

The nanofiber results emphasize the conclusions from the wing scale section. The blurred image regions could be compensated for with the Scheimpflug architecture [139], and the SNR could be improved with a different light source or a real-time feedback algorithm for controlling camera settings. 


\section{Conclusion and future work}

\subsection{Conclusion}

The motivation for this work was to use the properties of light, such as wavelength, polarization, and intensity, for inferring the structural organization of a medium. Probing a medium with polarized light can reveal structural organization features such as optical anisotropies, while the spectral aspect of polarization is sensitive to smallscale structure. Additionally, liquid crystal-based polarization devices allow for rapid, automated polarization measurements at multiple wavelengths. Thus, performing measurements with a liquid crystal-based spectral imaging polarimeter can reveal structural organization features by providing quantitative 2D maps of the complete spectral polarization response of a medium.

This dissertation described a methodology for elucidating structural organization, and consisted of a description of the conceptual framework, measurement system, and measurement interpretation. The conceptual framework included the Stokes/Mueller formalism, which describes the polarization response of a medium in

terms of optical anisotropies. For the measurement system, a complete account of 
system validation was given, where the validation was achieved through a series of calibrations, simulations, test measurements, and measurements on a rock thin section. Finally, measurement interpretation involved determining the validity of the results, and also relating the results to physical features of the sample. Several significant items were completed as part of this dissertation, and they were the following:

1. Developed a methodology for inferring structural organization. This consisted of the construction and validation of a multi-dimensional system, along with measurement interpretation. The system was the first of its kind to use four LCVRs and an LCTF, and could image the complete Mueller matrix as a function of wavelength. Most calibration and test measurements yielded less than $5 \%$ error for all Mueller matrix elements for a wavelength range of $500-720$ nm. Additionally, mineral structural organization was parameterized in terms of an effective birefringence and fast axis orientation.

2. Developed a new method for error analysis. Polarimetric measurement systems employing LCVRs are sensitive to alignment and temperature uncertainties. Through a series of simulations, a new error analysis method was developed that mapped specific LCVR uncertainties to error in specific Mueller matrix elements.

3. Characterized LCVR effects. Nematic liquid crystals inside the LCVRs exhibit a distinct structural organization, but also scatter a small amount of light. 
LCVR structural organization parameters were inferred from spectral scatter measurements. Additionally, these measurements served as a component of system performance analysis.

4. Optimized the measurement system. The simulation architecture from the error analysis method was also used for optimizing the system. Optimization was in terms of determining the proper tuning curve region for the LCVRs, along with the proper training set.

\subsection{Future work}

The spectral imaging goniometric polarimeter accurately functions in transmission mode, but has limitations in the backscatter mode. Addressing these limitations can serve as areas of future research. Furthermore, the system has the potential for various applications across multi-disciplinary fields.

1. Modify system to incorporate the Scheimpflug architecture. The shallow depth of field of the imaging objective (on the order of microns) results in a narrow region of focus when imaging at oblique angles. When the image has a surface that acts like a series of point scatterers, this effect worsens. The Scheimpflug architecture is expected to compensate for the narrow region of focus, as this architecture can be used in imaging systems in which either the 
object plane, image plane, or imaging lens are not parallel with each other. System modification would include incorporating pivoting optic mounts for either the objective lenses, the camera, or any combination of the three.

2. Implement a real-time feedback algorithm for controlling camera settings. For a given wavelength, the six Stokes vector measurements were all made at the same camera integration setting. This resulted in regions within the sample images that suffered from low SNR, caused by absorption, scattering, or both. However, due to the linearity of the camera exposure time, a real-time feedback algorithm for optimizing exposure time can be implemented. This algorithm would consist of real-time adjustment of the exposure time for each of the six Stokes vector measurements. Ultimately, the individual measurements would take advantage of the dynamic range of the camera, and measurements would be less susceptible to noise.

3. Measure spectral polarization response from other samples. The system can be used for making measurements on other types of media. This can include transmission or backscatter measurements on samples from the fields of biology, biomedicine, mineralogy, chemistry, and metrology. 


\subsection{Contributions}

The work in this dissertation has contributed to the polarimetry community through various conference presentations, proceedings, and posters, along with two journal publications. In total, ten different works have been produced as a result of this research. Below is a full list of these contributions.

\section{Journal publications}

1. J. C. Gladish and D. D. Duncan, "Alignment and temperature effects in liquid crystal-based active polarimetry," Applied Optics, vol. 53, no. 18, pp. 39823992, June 2014.

2. J. C. Gladish and D. D. Duncan, "Parameterizing LCVR structural organization with a fractal-Born approximation model," to be submitted to the Journal of the Optical Society of America A, May 2015.

\section{Conference proceedings and presentations}

3. J. C. Gladish and D. D. Duncan, "Liquid crystal-based spectral imaging goniometric polarimeter for sample characterization," Proceedings of SPIE Vol. 8942, Dynamics and Fluctuations in Biomedical Photonics XI, 894206 (2014).

4. J. C. Gladish and D. D. Duncan, "Spectral imaging polarimeter based on liquid 
crystal technology," Proceedings of SPIE Vol. 8222, Dynamics and Fluctuations in Biomedical Photonics IX, 82220X (2012).

5. J. C. Gladish and D. D. Duncan, "LCVR-based polarimetry: calibration issues," Proceedings of SPIE Vol. 7566, Optics in Tissue Engineering and Regenerative Medicine IV, 756609 (2010).

6. J. C. Gladish and D. D. Duncan, "Spectral polarimetry for assessing cell alignment in cultured tissues," Proceedings of SPIE Vol. 7179, Optics in Tissue Engineering and Regenerative Medicine III, 717909 (2009).

7. D. D. Duncan, J. C. Gladish, and B. D. Markway, "Tissue morphology from spectral polarimetry," Proceedings of SPIE Vol. 6858, Optics in Tissue Engineering and Regenerative Medicine II, 685809 (2008).

\section{Conference presentations}

8. J. C. Gladish and D. D. Duncan, "Scatter characteristics of liquid crystal variable retarders," 8580-9 in Dynamics and Fluctuations in Biomedical Photonics VII, (2013).

9. J. C. Gladish and D. D. Duncan, "Optimum polarimeter training sets for liquid crystal variable retarders," 8580-37 in Dynamics and Fluctuations in Biomedical Photonics VII, (2013). 


\section{Conference posters}

10. J. C. Gladish and D. D. Duncan, "Characterization of liquid crystal variable retarder scatter," 8222-39 in Dynamics and Fluctuations in Biomedical Photonics IX (2012). 


\section{References}

[1] H. van de Hulst, Light scattering by small particles. New York: Dover Publications, Inc., 1957.

[2] J. S. Tyo, D. L. Goldstein, D. B. Chenault, and J. A. Shaw, "Review of passive imaging polarimetry for remote sensing applications," Appl. Opt., vol. 45, no. 22, pp. 5453-5469, Aug 2006.

[3] H. Fang, M. Ollero, E. Vitkin, L. Kimerer, P. B. Cipolloni, M. Zaman, S. Freedman, I. Bigio, I. Itzkan, E. B. Hanlon, and L. T. Perelman, "Noninvasive sizing of subcellular organelles with light scattering spectroscopy," Selected Topics in Quantum Electronics, IEEE Journal of, vol. 9, no. 2, pp. 267-276, March 2003.

[4] D. D. Duncan and M. E. Thomas, "Particle shape as revealed by spectral depolarization," Appl. Opt., vol. 46, no. 24, pp. 6185-6191, Aug 2007.

[5] S. Asano, "Light scattering properties of spheroidal particles," Appl. Opt., vol. 18, no. 5, pp. 712-723, Mar 1979.

[6] S. Asano and M. Sato, "Light scattering by randomly oriented spheroidal particles," Appl. Opt., vol. 19, no. 6, pp. 962-974, Mar 1980.

[7] M. I. Mishchenko and J. W. Hovenier, "Depolarization of light backscattered by randomly oriented nonspherical particles," Opt. Lett., vol. 20, no. 12, pp. 1356-1358, Jun 1995.

[8] J. D. Keener, K. J. Chalut, J. W. Pyhtila, and A. Wax, "Application of Mie theory to determine the structure of spheroidal scatterers in biological materials," Opt. Lett., vol. 32, no. 10, pp. 1326-1328, May 2007.

[9] J. D. Wilson and T. H. Foster, "Mie theory interpretations of light scattering from intact cells," Opt. Lett., vol. 30, no. 18, pp. 2442-2444, Sep 2005.

[10] J. S. Baba, J.-R. Chung, A. H. DeLaughter, B. D. Cameron, and G. L. Cote, "Development and calibration of an automated Mueller matrix polarization imaging system," Journal of Biomedical Optics, vol. 7, no. 3, pp. 341-349, 2002 . 
[11] P. Ghassemi, P. Lemaillet, T. A. Germer, J. W. Shupp, S. S. Venna, M. E. Boisvert, K. E. Flanagan, M. H. Jordan, and J. C. Ramella-Roman, "Out-ofplane Stokes imaging polarimeter for early skin cancer diagnosis," Journal of Biomedical Optics, vol. 17, no. 7, pp. 076 014-1-076014-9, 2012.

[12] Y. Zhao, L. Zhang, and Q. Pan, "Spectropolarimetric imaging for pathological analysis of skin," Appl. Opt., vol. 48, no. 10, pp. D236-D246, Apr 2009.

[13] A. Pierangelo, S. Manhas, A. Benali, C. Fallet, J.-L. Totobenazara, M.-R. Antonelli, T. Novikova, B. Gayet, A. De Martino, and P. Validire, "Multispectral Mueller polarimetric imaging detecting residual cancer and cancer regression after neoadjuvant treatment for colorectal carcinomas," Journal of Biomedical Optics, vol. 18, no. 4, pp. $046014-1-046014-9,2013$.

[14] J. S. Tyo and T. S. Turner, "Variable-retardance, fourier-transform imaging spectropolarimeters for visible spectrum remote sensing," Appl. Opt., vol. 40, no. 9, pp. 1450-1458, Mar 2001.

[15] N. J. Pust and J. A. Shaw, "Dual-field imaging polarimeter using liquid crystal variable retarders," Appl. Opt., vol. 45, no. 22, pp. 5470-5478, Aug 2006.

[16] N. A. Beaudry, Y. Zhao, and R. Chipman, "Dielectric tensor measurement from a single Mueller matrix image," J. Opt. Soc. Am. A, vol. 24, no. 3, pp. 814-824, Mar 2007.

[17] S. Ben Hatit, M. Foldyna, A. De Martino, and B. Drvillon, "Angle-resolved Mueller polarimeter using a microscope objective," physica status solidi (a), vol. 205, no. 4, pp. 743-747, 2008.

[18] I. S. Nerbø, M. Kildemo, S. L. Roy, I. Simonsen, E. Søndergård, L. Holt, and J. C. Walmsley, "Characterization of nanostructured GaSb: comparison between large-area optical and local direct microscopic techniques," Appl. Opt., vol. 47, no. 28, pp. 5130-5139, Oct 2008.

[19] R. Chipman, "Chapter 22, Polarimetry," in Handbook of Optics, Volume II. New York, McGraw-Hill, Inc., 1995.

[20] J. W. Goodman, Statistical Optics. New York: John Wiley and Sons, Inc., 2000 .

[21] J. Beeckman, K. Neyts, and P. J. M. Vanbrabant, "Liquid-crystal photonic applications," Optical Engineering, vol. 50, no. 8, pp. 0812 021-08 120 217, 2011.

[22] M. Srinivasarao, "Nano-optics in the biological world: beetles, butterflies, birds, and moths," Chemical Reviews, vol. 99, no. 7, pp. 1935-1962, 1999. 
[23] K. Yu, T. Fan, S. Lou, and D. Zhang, "Biomimetic optical materials: Integration of natures design for manipulation of light," Progress in Materials Science, vol. 58, no. 6, pp. $825-873,2013$.

[24] P. Vukusic, "Structural colour: elusive iridescence strategies brought to light," Current Biology, vol. 21, no. 5, pp. R187-R189, 2011.

[25] R. W. Hart and R. A. Farrell, "Light scattering in the cornea," Journal of the Optical Society of America, vol. 59, pp. 766-774, June 1969.

[26] P. Vukusic, R. Sambles, C. Lawrence, and G. Wakely, "Sculpted-multilayer optical effects in two species of papilio butterfly," Appl. Opt., vol. 40, no. 7, pp. 1116-1125, Mar 2001.

[27] C. Knupp, C. Pinali, P. N. Lewis, G. J. Parfitt, R. D. Young, K. M. Meek, and A. J. Quantock, "The architecture of the cornea and structural basis of its transparency," in Advances in Protein Chemistry and Structural Biology, ser. Advances in Protein Chemistry and Structural Biology, A. McPherson, Ed. Academic Press, 2009, vol. 78, pp. 25 - 49.

[28] T. B. Smith, "Modeling corneal transparency," American Journal of Physics, vol. 75 , no. $7,2007$.

[29] N.-I. International, "Hybrid cornea," May 2014. [Online]. Available: http://www.hybridcornea.org/aboutcornea.htm

[30] L. Blog, "Lasik blog: All about lasik eye surgery in simple words," May 2014. [Online]. Available: http://lasikblog.net/photo-video/

[31] Olympus, "Optical birefringence," April 2015. [Online]. Available: http: //www.olympusmicro.com/primer/lightandcolor/birefringence.html

[32] Y. Chen, J. Gu, S. Zhu, T. Fan, D. Zhang, and Q. Guo, "Iridescent large-area $\mathrm{ZrO} 2$ photonic crystals using butterfly as templates," Applied Physics Letters, vol. 94, no. 5, pp. $0539011-0539013,2009$.

[33] Huang, WangWang, and Z. L. Wang, "Controlled replication of butterfly wings for achieving tunable photonic properties," Nano Letters, vol. 6, no. 10, pp. $2325-2331,2006$.

[34] J. Hwang, M. H. Song, B. Park, S. Nishimura, T. Toyooka, J. W. Wu, Y. Takanishi, K. Ishikawa, and H. Takezoe, "Electro-tunable optical diode based on photonic bandgap liquid-crystal heterojunctions," Nature Materials, vol. 4, no. 5, pp. 383-387, 2005. 
[35] G. Pfaff and P. Reynders, "Angle-dependent optical effects deriving from submicron structures of films and pigments," Chemical Reviews, vol. 99, no. 7, pp. 1963-1982, 1999.

[36] C. Palmer, Diffraction Grating Handbook. Thermo RGL, New York, 2002.

[37] J. Dobrowolski, "Chapter 42, Optical Properties of Films and Coatings," in Handbook of Optics, Volume I. New York, McGraw-Hill, Inc., 1995.

[38] J. Bennett, "Chapter 3, Polarizers," in Handbook of Optics, Volume II. New York, McGraw-Hill, Inc., 1995.

[39] C. Brosseau, Fundamentals of polarized light, A statistical optics approach. New York: John Wiley and Sons, Inc., 1998.

[40] D. Goldstein, Polarized Light. New York: Marcel Dekker, Inc., 2003.

[41] J. Schott, Fundamentals of Polarimetric Remote Sensing. Bellingham, Washington: Society for Photo-Optical Instrumentation Engineers, 2009.

[42] T. J. H. William J. Tropf, Michael E. Thomas, "Chapter 33, Properties of Crystals and Glasses," in Handbook of Optics, Volume II. New York, McGrawHill, Inc., 1995.

[43] D. B. Murphy, Fundamentals of light microscopy and electronic imaging, 1st ed. Wiley-Liss, 2001.

[44] J. Stover, Optical Scattering: Measurement and Analysis. New York: McGrawHill, Inc., 1990.

[45] E. Garcia-Caurel, A. D. Martino, J.-P. Gaston, and L. Yan, "Application of spectroscopic ellipsometry and Mueller ellipsometry to optical characterization," Appl. Spectrosc., vol. 67, no. 1, pp. 1-21, Jan 2013.

[46] M. Losurdo, M. Bergmair, G. Bruno, D. Cattelan, C. Cobet, A. de Martino, K. Fleischer, Z. Dohcevic-Mitrovic, N. Esser, M. Galliet, R. Gajic, D. Hemzal, K. Hingerl, J. Humlicek, R. Ossikovski, Z. Popovic, and O. Saxl, "Spectroscopic ellipsometry and polarimetry for materials and systems analysis at the nanometer scale: state-of-the-art, potential, and perspectives," Journal of Nanoparticle Research, vol. 11, no. 7, pp. 1521-1554, 2009.

[47] C. Deumié, H. Giovannini, and C. Amra, "Angle-resolved ellipsometry of light scattering: discrimination of surface and bulk effects in substrates and optical coatings," Appl. Opt., vol. 41, no. 16, pp. 3362-3369, Jun 2002. 
[48] T. A. Germer, "Angular dependence and polarization of out-of-plane optical scattering from particulate contamination, subsurface defects, and surface microroughness," Appl. Opt., vol. 36, no. 33, pp. 8798-8805, Nov 1997.

[49] T. A. Germer and C. C. Asmail, "Polarization of light scattered by microrough surfaces and subsurface defects," J. Opt. Soc. Am. A, vol. 16, no. 6, pp. 13261332, Jun 1999.

[50] T. A. Germer and E. Marx, "Ray model of light scattering by flake pigments or rough surfaces with smooth transparent coatings," Appl. Opt., vol. 43, no. 6, pp. 1266-1274, Feb 2004.

[51] P. Terrier, V. Devlaminck, and J. M. Charbois, "Segmentation of rough surfaces using a polarization imaging system," J. Opt. Soc. Am. A, vol. 25, no. 2, pp. 423-430, Feb 2008.

[52] J. Shen, D. Deng, W. Kong, S. Liu, Z. Shen, C. Wei, H. He, J. Shao, and Z. Fan, "Extended bidirectional reflectance distribution function for polarized light scattering from subsurface defects under a smooth surface," J. Opt. Soc. Am. A, vol. 23, no. 11, pp. 2810-2816, Nov 2006.

[53] I. G. E. Renhorn, T. Hallberg, D. Bergström, and G. D. Boreman, "Fourparameter model for polarization-resolved rough-surface BRDF," Opt. Express, vol. 19, no. 2, pp. 1027-1036, Jan 2011.

[54] D. D. Duncan, D. V. Hahn, and M. E. Thomas, "Physics-based polarimetric BRDF models," Proc. SPIE 5192, Optical Diagnostic Methods for Inorganic Materials III, pp. 129-140, 2003.

[55] J. E. Harvey, A. Krywonos, and C. L. Vernold, "Modified Beckmann-Kirchhoff scattering model for rough surfaces with large incident and scattering angles," Optical Engineering, vol. 46, no. 7, pp. 078 002-1-078002-10, 2007.

[56] M. E. McKnight, T. V. Vorburger, E. Marx, M. E. Nadal, P. Y. Barnes, and M. A. Galler, "Measurements and predictions of light scattering by clear coatings," Appl. Opt., vol. 40, no. 13, pp. 2159-2168, May 2001.

[57] A. Ferrero, A. M. Rabal, J. Campos, A. Pons, and M. L. Hernanz, "Variables separation of the spectral BRDF for better understanding color variation in special effect pigment coatings," J. Opt. Soc. Am. A, vol. 29, no. 6, pp. 842847, Jun 2012.

[58] A. Krywonos, J. E. Harvey, and N. Choi, "Linear systems formulation of scattering theory for rough surfaces with arbitrary incident and scattering angles," J. Opt. Soc. Am. A, vol. 28, no. 6, pp. 1121-1138, Jun 2011. 
[59] A. Laskarakis, S. Logothetidis, E. Pavlopoulou, and M. Gioti, "Mueller matrix spectroscopic ellipsometry: formulation and application," Thin Solid Films, vol. 455, pp. 43 - 49, 2004, the 3rd International Conference on Spectroscopic Ellipsometry.

[60] B. H. Ibrahim, S. B. Hatit, and A. D. Martino, "Angle resolved Mueller polarimetry with a high numerical aperture and characterization of transparent biaxial samples," Appl. Opt., vol. 48, no. 27, pp. 5025-5034, Sep 2009.

[61] T. Novikova, A. D. Martino, S. B. Hatit, and B. Drévillon, "Application of Mueller polarimetry in conical diffraction for critical dimension measurements in microelectronics," Appl. Opt., vol. 45, no. 16, pp. 3688-3697, Jun 2006.

[62] K. M. Twietmeyer, R. A. Chipman, A. E. Elsner, Y. Zhao, and D. VanNasdale, "Mueller matrix retinal imager with optimized polarization conditions," Opt. Express, vol. 16, no. 26, pp. 21 339-21 354, Dec 2008.

[63] J. S. Tyo, "Design of optimal polarimeters: maximization of signal-to-noise ratio and minimization of systematic error," Appl. Opt., vol. 41, no. 4, pp. 619-630, Feb 2002.

[64] J. S. Tyo, M. P. Rowe, E. N. Pugh, and N. Engheta, "Target detection in optically scattering media by polarization-difference imaging," Appl. Opt., vol. 35, no. 11, pp. 1855-1870, Apr 1996.

[65] R. W. Collins and Y. T. Kim, "Ellipsometry for thin-film and surface analysis," Analytical Chemistry, vol. 62, no. 17, pp. 887A-890A, 1990.

[66] M. H. Smith, J. B. Woodruff, and J. D. Howe, "Beam wander considerations in imaging polarimetry," Proc. SPIE 3754, Polarization: Measurement, Analysis, and Remote Sensing II, pp. 50-54, 1999.

[67] M. H. Smith, J. D. Howe, J. B. Woodruff, M. A. Miller, G. R. Ax, Jr., T. E. Petty, and E. A. Sornsin, "Multispectral infrared Stokes imaging polarimeter," Proc. SPIE 3754, Polarization: Measurement, Analysis, and Remote Sensing II, pp. 137-143, 1999.

[68] M. H. Smith, "Optimization of a dual-rotating-retarder Mueller matrix polarimeter," Appl. Opt., vol. 41, no. 13, pp. 2488-2493, May 2002.

[69] J. C. Ramella-Roman and D. D. Duncan, "A new approach to Mueller matrix reconstruction of skin cancer lesions using a dual rotating retarder polarimeter," Proc. SPIE 6080, Advanced Biomedical and Clinical Diagnostic Systems IV, pp. 60 800M-1-60 800M-7, 2006. 
[70] D. S. Sabatke, M. R. Descour, E. L. Dereniak, W. C. Sweatt, S. A. Kemme, and G. S. Phipps, "Optimization of retardance for a complete Stokes polarimeter," Opt. Lett., vol. 25, no. 11, pp. 802-804, Jun 2000.

[71] D. H. Goldstein, "Mueller matrix dual-rotating retarder polarimeter," Appl. Opt., vol. 31, no. 31, pp. 6676-6683, Nov 1992.

[72] G. Martnez-Ponce, C. Solano, and C. Prez-Barrios, "Hybrid complete Mueller polarimeter based on phase modulators," Optics and Lasers in Engineering, vol. 49, no. 6, pp. $723-728,2011$, research in Optics and Photonics at CIO, Mexico.

[73] X. Guo, M. F. G. Wood, and I. A. Vitkin, "Angular measurements of light scattered by turbid chiral media using linear Stokes polarimeter," Journal of Biomedical Optics, vol. 11, no. 4, pp. 041 105-1-041 105-10, 2006.

[74] O. Arteaga, J. Freudenthal, B. Wang, and B. Kahr, "Mueller matrix polarimetry with four photoelastic modulators: theory and calibration," Appl. Opt., vol. 51, no. 28, pp. 6805-6817, Oct 2012.

[75] S. Nichols, J. Freudenthal, O. Arteaga, and B. Kahr, "Imaging with photoelastic modulators," Proc. SPIE 9099, Polarization: Measurement, Analysis, and Remote Sensing XI, pp. 909 912-1-909 912-10, 2014.

[76] S. Alali, T. Yang, and I. A. Vitkin, "Rapid time-gated polarimetric Stokes imaging using photoelastic modulators," Opt. Lett., vol. 38, no. 16, pp. 29973000, Aug 2013.

[77] S. Alali and I. A. Vitkin, "Optimization of rapid Mueller matrix imaging of turbid media using four photoelastic modulators without mechanically moving parts," Optical Engineering, vol. 52, no. 10, pp. 103114-1-103114-8, 2013.

[78] K. Oka and T. Kaneko, "Compact complete imaging polarimeter using birefringent wedge prisms," Opt. Express, vol. 11, no. 13, pp. 1510-1519, Jun 2003.

[79] Q. Cao, C. Zhang, and E. DeHoog, "Snapshot imaging polarimeter using modified Savart polariscopes," Appl. Opt., vol. 51, no. 24, pp. 5791-5796, Aug 2012.

[80] T. Mu, C. Zhang, and B. Zhao, "Principle and analysis of a polarization imaging spectrometer," Appl. Opt., vol. 48, no. 12, pp. 2333-2339, Apr 2009.

[81] C. Zhang, H. Wu, and J. Li, "Fourier transform hyperspectral imaging polarimeter for remote sensing," Optical Engineering, vol. 50, no. 6, pp. $066201-$ 1-066 201-5, 2011. 
[82] J. Craven-Jones, M. W. Kudenov, M. G. Stapelbroek, and E. L. Dereniak, "Infrared hyperspectral imaging polarimeter using birefringent prisms," Appl. Opt., vol. 50, no. 8, pp. 1170-1185, Mar 2011.

[83] C. Oh and M. J. Escuti, "Achromatic diffraction from polarization gratings with high efficiency," Opt. Lett., vol. 33, no. 20, pp. 2287-2289, Oct 2008.

[84] M. W. Kudenov, M. J. Escuti, E. L. Dereniak, and K. Oka, "White-light channeled imaging polarimeter using broadband polarization gratings," Appl. Opt., vol. 50, no. 15, pp. 2283-2293, May 2011.

[85] M. W. Kudenov, M. J. Escuti, N. Hagen, E. L. Dereniak, and K. Oka, "Snapshot imaging Mueller matrix polarimeter using polarization gratings," Opt. Lett., vol. 37, no. 8, pp. 1367-1369, Apr 2012.

[86] F. Goudail, P. Terrier, Y. Takakura, L. Bigué, F. Galland, and V. DeVlaminck, "Target detection with a liquid-crystal-based passive Stokes polarimeter," Appl. Opt., vol. 43, no. 2, pp. 274-282, Jan 2004.

[87] E. Garcia-Caurel, A. D. Martino, and B. Drvillon, "Spectroscopic Mueller polarimeter based on liquid crystal devices," Thin Solid Films, vol. 455, pp. 120 - 123, 2004, the 3rd International Conference on Spectroscopic Ellipsometry.

[88] B. Laude-Boulesteix, A. D. Martino, B. Drévillon, and L. Schwartz, "Mueller polarimetric imaging system with liquid crystals," Appl. Opt., vol. 43, no. 14, pp. 2824-2832, May 2004.

[89] A. Peinado, A. Lizana, and J. Campos, "Optimization and tolerance analysis of a polarimeter with ferroelectric liquid crystals," Appl. Opt., vol. 52, no. 23, pp. 5748-5757, Aug 2013.

[90] A. Peinado, A. Lizana, J. Vidal, C. Iemmi, and J. Campos, "Optimization and performance criteria of a Stokes polarimeter based on two variable retarders," Opt. Express, vol. 18, no. 10, pp. 9815-9830, May 2010.

[91] A. D. Martino, Y.-K. Kim, E. Garcia-Caurel, B. Laude, and B. Drévillon, "Optimized Mueller polarimeter with liquid crystals," Opt. Lett., vol. 28, no. 8, pp. 616-618, Apr 2003.

[92] J. C. Ramella-Roman, A. Nayak, and S. A. Prahl, "Spectroscopic sensitive polarimeter for biomedical applications," Journal of Biomedical Optics, vol. 16, no. 4, pp. $047001-1-047001-7,2011$.

[93] B. Boulbry, J. C. Ramella-Roman, and T. A. Germer, "Improved method for calibrating a Stokes polarimeter," Appl. Opt., vol. 46, no. 35, pp. 8533-8541, Dec 2007. 
[94] Y. Zhang, H. Zhao, and N. Li, "Polarization calibration with large apertures in full field of view for a full Stokes imaging polarimeter based on liquid-crystal variable retarders," Appl. Opt., vol. 52, no. 6, pp. 1284-1292, Feb 2013.

[95] J. S. Tyo, "Noise equalization in Stokes parameter images obtained by use of variable-retardance polarimeters," Opt. Lett., vol. 25, no. 16, pp. 1198-1200, Aug 2000.

[96] J. M. Bueno, "Polarimetry using liquid-crystal variable retarders: theory and calibration," Journal of Optics A: Pure and Applied Optics, vol. 2, no. 3, pp. 216-222, 2000.

[97] W. A. Woźniak, P. Kurzynowski, and S. Drobczyński, "Adjustment method of an imaging Stokes polarimeter based on liquid crystal variable retarders," Appl. Opt., vol. 50, no. 2, pp. 203-212, Jan 2011.

[98] K. M. Twietmeyer and R. A. Chipman, "Optimization of Mueller matrix polarimeters in the presence of error sources," Opt. Express, vol. 16, no. 15, pp. 11 589-11603, Jul 2008.

[99] M. Francon and S. Mallick, Polarization Interferometers. Belgium: John Wiley and Sons Ltd., 1971.

[100] M. W. Kudenov, M. E. L. Jungwirth, E. L. Dereniak, and G. R. Gerhart, "White light sagnac interferometer for snapshot linear polarimetric imaging," Opt. Express, vol. 17, no. 25, pp. 22 520-22 534, Dec 2009.

[101] A. Martínez, N. Beaudoin, I. Moreno, M. del Mar Sánchez-López, and P. Velásquez, "Optimization of the contrast ratio of a ferroelectric liquid crystal optical modulator," Journal of Optics A: Pure and Applied Optics, vol. 8, no. 11, pp. 1013-1018, 2006.

[102] E. Compain, S. Poirier, and B. Drevillon, "General and self-consistent method for the calibration of polarization modulators, polarimeters, and Mueller-matrix ellipsometers," Appl. Opt., vol. 38, no. 16, pp. 3490-3502, Jun 1999.

[103] A. Ambirajan and D. C. Look, Jr., "Optimum angles for a polarimeter: part I," Optical Engineering, vol. 34, no. 6, pp. 1651-1655, 1995.

[104] — - "Optimum angles for a polarimeter: part II," Optical Engineering, vol. 34, no. 6, pp. 1656-1658, 1995.

[105] T. K. Moon and W. C. Stirling, Mathematical methods and algorithms for signal processing. Upper Saddle River, New Jersey: Prentice Hall, 2000.

[106] G. H. Golub and C. F. V. Loan, Matrix Computations, Second Edition. Johns Hopkins U. Press, 1989. 
[107] M. J. Stephen and J. P. Straley, "Physics of liquid crystals," Rev. Mod. Phys., vol. 46, pp. 617-704, Oct 1974.

[108] P. J. Collings, Liquid Crystals: Nature's Delicate Phase of Matter. Princeton: Princeton University Press, 2002.

[109] D3040 Quad Cell Nematic Liquid Crystal Digital Interface User's Manual, Meadowlark Optics, 2004.

[110] P. G. deGennes and J. Prost, The physics of liquid crystals, second edition. New York: Oxford University Press, 1993.

[111] X. Xiao and D. G. Voelz, "Liquid crystal variable retarder modeling of incident angle response with experimental verification," Optical Engineering, vol. 47, no. 5, pp. 054002-1-054002-8, 2008.

[112] CRI, "Technical note: FAQs and principles of operation," Woburn, MA: Cambridge Research and Instrumentation, Inc., Tech. Rep.

[113] VariSpec Liquid Crystal Tunable Filters User's Manual, Woburn, MA: Cambridge Research and Instrumentation, Inc., 2004.

[114] J. Mertz, Introduction to Optical Microscopy. Greenwood Village, Colorado: Roberts and Company Publishers, 2010.

[115] S.-T. Wu, U. Efron, and L. D. Hess, "Birefringence measurements of liquid crystals," Appl. Opt., vol. 23, no. 21, pp. 3911-3915, Nov 1984.

[116] N. Uribe-Patarroyo, A. Alvarez-Herrero, and V. M. Pillet, "Preflight calibration of the imaging magnetograph experiment polarization modulation package based on liquid-crystal variable retarders," Appl. Opt., vol. 51, no. 21, pp. 49544970, Jul 2012.

[117] X. Xiao, D. G. Voelz, and H. Sugiura, "Field-of-view characteristics of a liquid crystal variable retarder," Proc. SPIE 5158, Polarization Science and Remote Sensing, pp. 142-150, 2003.

[118] E. W. Weisstein, "Sphere point picking," From MathWorld-A Wolfram Web Resource, created by Eric W. Weisstein, January 2013, http://mathworld. wolfram.com/SpherePointPicking.html.

[119] T. Rowland, "Spherical distance," From MathWorld-A Wolfram Web Resource, created by Eric W. Weisstein, January 2013, http://mathworld.wolfram.com/ SphericalDistance.html.

[120] J. W. Goodman, Introduction to Fourier Optics, 3rd ed. Englewood, Colorado: Roberts and Company, 2005. 
[121] Meadowlark Optics catalog, Meadowlark Optics, 2008.

[122] D. Cote and I. A. Vitkin, "Balanced detection for low-noise precision polarimetric measurements of optically active, multiply scattering tissue phantoms," Journal of Biomedical Optics, vol. 9, no. 1, pp. 213-220, 2004.

[123] R. J. McNichols and G. L. Cote, "Optical glucose sensing in biological fluids: an overview," Journal of Biomedical Optics, vol. 5, no. 1, pp. 5-16, 2000.

[124] C. A. Browne and F. W. Zerban, Physical and Chemical Methods of Sugar Analysis, 3rd edition. John Wiley and Sons, Inc., New York, 1941.

[125] R. R. Ansari, S. Bockle, and L. Rovati, "New optical scheme for a polarimetricbased glucose sensor," Journal of Biomedical Optics, vol. 9, no. 1, pp. 103-115, 2004 .

[126] W. H. Carter, "Chapter 4, Coherence Theory," in Handbook of Optics, Volume I. New York, McGraw-Hill, Inc., 1995.

[127] E. Wolf, Introduction to the theory of coherence and polarization of light. Cambridge: University Press, 2007.

[128] J. C. Gladish and D. D. Duncan, "Alignment and temperature effects in liquid crystal-based active polarimetry," Applied Optics, vol. 53, pp. 3982-3992, June 2014.

[129] M. Born and E. Wolf, Principles of Optics. New York: Cambridge University Press, 1999.

[130] V. I. Tatarski, Wave Propagation in a Turbulent Medium. New York: Dover Publications, Inc., 1961.

[131] M. Hunter, V. Backman, G. Popescu, M. Kalashnikov, C. W. Boone, A. Wax, V. Gopal, K. Badizadegan, G. D. Stoner, and M. S. Feld, "Tissue self-affinity and polarized light scattering in the Born approximation: A new model for precancer detection," Phys. Rev. Lett., vol. 97, pp. 138102-1-138102-4, Sep 2006.

[132] P. Banerjee, J. Soni, H. Purwar, N. Ghosh, and T. K. Sengupta, "Probing the fractal pattern and organization of bacillus thuringiensis bacteria colonies growing under different conditions using quantitative spectral light scattering polarimetry," Journal of Biomedical Optics, vol. 18, no. 3, pp. 035 003-1-035 00310, 2013.

[133] R. F. Voss, "Random Fractal Forgeries," in Fundamental Algorithms for Computer Graphics, R. Earnshaw, Ed. Springer-Verlag, Berlin, 1985. 
[134] J. Goldstein, Scanning electron microscopy and X-ray microanalysis, Second edition. New York: Plenum Press, 1992.

[135] P. F. Kerr, Optical Mineralogy. New York: McGraw-Hill Book Company, Inc., 1959.

[136] J. W. Anthony and R. A. Bideaux, Handbook of Mineralogy, Volume 2, Silica, Silicates. Arizona: Mineral Data Publishing, 1995.

[137] W. S. MacKenzie and A. E. Adams, A Color Atlas of Rocks and Minerals in Thin Sections. New York: John Wiley and Sons, Inc., 1997.

[138] J. W. Anthony and R. A. Bideaux, Handbook of Mineralogy, Volume 4, Arsenates, Phosphates, Vanadates. Arizona: Mineral Data Publishing, 1995.

[139] R. Drews, "Depth of field in slit lamp photography," Ophthalmologica, vol. 148, pp. 143-150, 1964.

[140] E. L. O’Neill, Introduction to Statistical Optics. Reading, Massachusetts: Addison-Wesley Publishing Company, 1963.

[141] A. M. Title and W. J. Rosenberg, "Tunable birefringent filters," Optical Engineering, vol. 20, no. 6, pp. 815-823, 1981.

[142] L. Mandel and E. Wolf, Optical coherence and quantum optics. New York: Cambridge University Press, 1995.

[143] P. J. Shull, Nondestructive Evaluation: Theory, Techniques, and Applications. New York: Marcel Dekker, Inc., 2002.

[144] D. G. Fischer, "Methods of inverse scattering for random media," Ph.D. dissertation, University of Rochester, 1996. 


\section{Appendix A Relating the Jones and Stokes/Mueller formalisms}

The Jones and Stokes/Mueller formalisms are two distinct, yet related, methods for describing the polarization state of an electromagnetic wave. Although they both describe polarization phenomena, they do so in different ways. The Jones formalism assumes the coherent addition of waves, and characterizes the field in terms of amplitude and phase. This formalism expresses the field and medium with complex vectors and matrices, respectively, and represents only fully polarized light and nondepolarizing systems. On the other hand, the Stokes/Mueller formalism assumes the incoherent addition of waves, and characterizes the field in terms of intensity. The field and medium are described by real vectors and matrices, respectively, and can represent any polarization state and depolarizing systems $[39,40]$. Consequently, the Jones formalism cannot account for depolarization and the Stokes/Mueller formalism cannot account for absolute phase [19]. Ultimately, this project uses the Mueller formalism, as this formalism employs an easily measurable parameter (intensity) and accounts for depolarization. This appendix begins by defining the Jones formalism, and then shows the relationship between the Jones formalism and the Stokes/Mueller formalism through use of the coherency matrix. 
The Jones formalism is a matrix method that describes how a medium linearly transforms an incident field into an output field. This transformation is in terms of complex vectors and matrices, where $2 \times 1$ vectors describe the fields and a $2 \times 2$ matrix represents the medium. The Jones vector $\varepsilon$ describes the incident field,

$$
\boldsymbol{\varepsilon}=\left[\begin{array}{c}
E_{x} \\
E_{y}
\end{array}\right],
$$

where $E_{x}$ and $E_{y}$ are the two orthogonal field components [140].

The Jones matrix $\mathbf{L}$ linearly transforms the incident field to an output field $\boldsymbol{\varepsilon}^{\prime}$,

$$
\varepsilon^{\prime}=\mathbf{L} \varepsilon
$$

where $\mathbf{L}$ describes the effects of an optical element or medium on the field,

$$
\mathbf{L}=\left[\begin{array}{ll}
l_{11}(\lambda) & l_{12}(\lambda) \\
l_{21}(\lambda) & l_{22}(\lambda)
\end{array}\right]
$$

When the system is comprised of multiple elements, $\mathbf{L}$ is the product of the Jones matrix representations of the individual elements, but multiplied in the reverse order that they appear in the system. For $N$ system components, $\mathbf{L}$ is expressed as

$$
\mathbf{L}=\mathbf{L}_{N} \mathbf{L}_{N-1} \ldots \mathbf{L}_{2} \mathbf{L}_{1} .
$$


Next, the coherency matrix can be defined. This matrix converts the field representation into a statistical representation. The coherency matrix $\mathbf{J}$ for a field is defined as

$$
\mathbf{J}=\left\langle\varepsilon \times \varepsilon^{\dagger}\right\rangle=\left[\begin{array}{cc}
\left\langle E_{x} E_{x}^{*}\right\rangle & \left\langle E_{x} E_{y}^{*}\right\rangle \\
\left\langle E_{y} E_{x}^{*}\right\rangle & \left\langle E_{y} E_{y}^{*}\right\rangle
\end{array}\right]=\left[\begin{array}{cc}
J_{x x} & J_{x y} \\
J_{y x} & J_{y y}
\end{array}\right],
$$

where $\times$ signifies the Kronecker product $[105],\langle\cdots\rangle$ represents the ensemble average, and $\boldsymbol{\varepsilon}^{\dagger}$ is the conjugate transpose [140],

$$
\varepsilon^{\dagger}=\left[\begin{array}{ll}
E_{x}^{*} & E_{y}^{*}
\end{array}\right]
$$

For the system output $\boldsymbol{\varepsilon}^{\prime}$, equations (A.2) and (A.5) can be used for calculating the coherency matrix $\mathbf{J}^{\prime}$ for the output, which simplifies to

$$
\mathbf{J}^{\prime}=\left\langle\varepsilon^{\prime} \times \varepsilon^{\prime \dagger}\right\rangle=\left\langle\mathbf{L} \varepsilon \times \varepsilon^{\dagger} \mathbf{L}^{\dagger}\right\rangle=\mathbf{L} \mathbf{J} \mathbf{L}^{\dagger} .
$$

The intensity of the field is simply the trace of the coherency matrix. For the outgoing field, the intensity $I^{\prime}$ is the sum of the $\mathbf{J}^{\prime}$ diagonal components [140],

$$
I^{\prime}=\operatorname{Tr} \mathbf{J}^{\prime}=J_{x x}^{\prime}+J_{y y}^{\prime}
$$

The coherency matrix can be used to relate the Jones formalism to the 
Stokes/Mueller formalism. The first step in establishing this relationship entails defining a column vector $\mathfrak{I}$ from the coherency matrix

$$
\mathfrak{I}=\left\langle\varepsilon \times \varepsilon^{*}\right\rangle=\left[\begin{array}{c}
J_{x x} \\
J_{x y} \\
J_{y x} \\
J_{y y}
\end{array}\right],
$$

where $*$ denotes the complex conjugate. The relationship linking the formalisms is

$$
\mathbf{S}=\mathbf{T} \mathfrak{I}
$$

which can also be expressed as

$$
\left[\begin{array}{l}
S_{1} \\
S_{2} \\
S_{3} \\
S_{4}
\end{array}\right]=\frac{1}{\sqrt{2}}\left[\begin{array}{cccc}
1 & 0 & 0 & 1 \\
1 & 0 & 0 & -1 \\
0 & 1 & 1 & 0 \\
0 & -i & i & 0
\end{array}\right]\left[\begin{array}{c}
J_{x x} \\
J_{x y} \\
J_{y x} \\
J_{y y}
\end{array}\right]
$$

This provides the transformation from the coherency matrix to the Stokes vector.

To find the output Stokes vector from an optical system, recall equations (A.2) and (A.7) such that

$$
\mathfrak{I}^{\prime}=\left\langle\varepsilon^{\prime} \times \varepsilon^{\prime *}\right\rangle=\left\langle\mathbf{L} \varepsilon \times \mathbf{L}^{*} \varepsilon^{*}\right\rangle .
$$


Using the relationship $\left(\mathbf{A A}^{\prime} \times \mathbf{B B}^{\prime}\right)=(\mathbf{A} \times \mathbf{B})\left(\mathbf{A}^{\prime} \times \mathbf{B}^{\prime}\right)$, equation (A.12) becomes

$$
\mathfrak{I}^{\prime}=\left\langle\left(\mathbf{L} \times \mathbf{L}^{*}\right)\left(\varepsilon \times \varepsilon^{*}\right)\right\rangle=\left\langle\mathbf{L} \times \mathbf{L}^{*}\right\rangle \mathfrak{I} .
$$

Now, using equation (A.10), it is straightforward to see that

$$
\mathbf{S}^{\prime}=\mathbf{T} \mathfrak{I}^{\prime}=\mathbf{T}\left(\mathbf{L} \times \mathbf{L}^{*}\right) \mathbf{T}^{-1} \mathbf{T} \mathfrak{I}=\left[\mathbf{T}\left(\mathbf{L} \times \mathbf{L}^{*}\right) \mathbf{T}^{-1}\right] \mathbf{S},
$$

and the Mueller matrix takes the form [140]

$$
\mathbf{M}=\mathbf{T}\left(\mathbf{L} \times \mathbf{L}^{*}\right) \mathbf{T}^{-1} .
$$

Thus, the output Stokes vector is given by

$$
\mathbf{S}^{\prime}=\left[\mathbf{T}\left(\mathbf{L} \times \mathbf{L}^{*}\right) \mathbf{T}^{-1}\right] \mathbf{S}=\mathbf{M S} .
$$

This derivation shows that equation (A.15) transforms a Jones matrix into a Mueller matrix. All Jones matrices can be mapped to a corresponding Mueller matrix. However, because phase is not preserved, the mapping is not invertible. It also should be noted that only non-depolarizing Mueller matrices have corresponding Jones matrices [19]. There is also a straightforward relationship between individual Mueller matrix elements and individual Jones matrix elements, given in [19]. Finally, 
in Appendix B are common Jones matrices and their Mueller matrix counterparts. 


\section{Appendix B Common Jones and Mueller matrices}

Jones and Mueller matrices describe the polarization-altering properties of an optical component or medium. This appendix describes how the Jones and Mueller matrices account for both polarization and orientation effects. First, Jones matrices are given, which are then subsequently converted to Mueller matrices using the transformations shown in Appendix A.

The waveplate, or retarder, is a commonly-used optical component that introduces a phase shift between the orthogonal components of the incident beam. The Jones matrix for a waveplate with its fast axis parallel to the $x$-axis of the laboratory reference frame is given by

$$
\mathbf{J}_{w}(\delta)=\left[\begin{array}{cc}
e^{i \delta / 2} & 0 \\
0 & e^{-i \delta / 2}
\end{array}\right],
$$

where $\delta$ is the phase shift (or retardance) between the $x$ - and $y$-components of the incident field.

Waveplates also produce polarization effects through a change in orientation. 


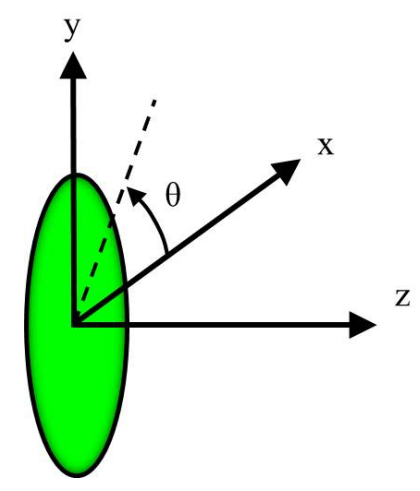

Figure B.1: Schematic representation of the rotation of an optical component through an angle $\theta$ about the $z$-axis.

Waveplate orientation refers to the angle between the fast axis and the $x$-axis of the laboratory reference frame. A waveplate, or any optical component, can be oriented at a specific angle $\theta$ relative to the $x$-axis of the laboratory reference frame (see Fig. B.1). The rotation can be accounted for in the Jones calculus with a construct called the rotator [140]. The rotator is a function of the orientation angle $\theta$, where $\theta$ describes a rotation about the $z$-axis (the propagation axis) in the $x$ - $y$ plane. Therefore, the component of interest needs to be rotated by the rotator within the Jones calculus.

The Jones matrix corresponding to rotation through an angle $\theta$ by a rotator is

$$
\mathbf{J}_{r o t}(\theta)=\left[\begin{array}{cc}
\cos \theta & \sin \theta \\
-\sin \theta & \cos \theta
\end{array}\right] \text {. }
$$

The new Jones matrix $\mathbf{J}(\theta)$ for a component $\mathbf{J}$ rotated through an angle $\theta$ is

$$
\mathbf{J}(\theta)=\mathbf{J}_{\text {rot }}(-\theta) \mathbf{J J}_{\text {rot }}(\theta)
$$


The general form of the Jones matrix for a waveplate at angle $\theta$ and retardance $\delta$ is found through use of equation (B.3),

$$
\mathbf{J}_{w}(\theta, \delta)=\mathbf{J}_{\text {rot }}(-\theta) \mathbf{J}_{w}(\delta) \mathbf{J}_{\text {rot }}(\theta),
$$

with the final result taking the form

$$
\mathbf{J}_{w}(\theta, \delta)=\left[\begin{array}{cc}
\cos \left(\frac{\delta}{2}\right)+i \sin \left(\frac{\delta}{2}\right) \cos 2 \theta & i \sin \left(\frac{\delta}{2}\right) \sin 2 \theta \\
i \sin \left(\frac{\delta}{2}\right) \sin 2 \theta & \cos \left(\frac{\delta}{2}\right)-i \sin \left(\frac{\delta}{2}\right) \cos 2 \theta
\end{array}\right]
$$

Another commonly employed element is the polarizer. The Jones matrix for a linear polarizer is,

$$
\mathbf{J}_{p}\left(k_{1}, k_{2}\right)=\left[\begin{array}{ll}
k_{1} & 0 \\
0 & k_{2}
\end{array}\right]
$$

where $k_{1}$ and $k_{2}$ are the principal field transmittances in the $x$ - and $y$-directions, respectively. When this element is oriented at an angle $\theta$ relative to the $x$-axis, its Jones matrix becomes

$$
\mathbf{J}_{p}\left(k_{1}, k_{2}, \theta\right)=\left[\begin{array}{cc}
k_{1} \cos ^{2} \theta+k_{2} \sin ^{2} \theta & k_{1} \cos \theta \sin \theta-k_{2} \cos \theta \sin \theta \\
k_{1} \cos \theta \sin \theta-k_{2} \cos \theta \sin \theta & k_{1} \sin ^{2} \theta+k_{2} \cos ^{2} \theta
\end{array}\right] \text {, }
$$


For an ideal linear polarizer, $k_{1}=1, k_{2}=0$, and the Jones matrix is [140]

$$
\mathbf{J}_{p}(\theta)=\left[\begin{array}{cc}
\cos ^{2} \theta & \cos \theta \sin \theta \\
\cos \theta \sin \theta & \sin ^{2} \theta
\end{array}\right] .
$$

The aforementioned Jones matrices can be converted to Mueller matrices using the transformation from Appendix A. For example, the rotator Jones matrix in equation (B.2) is expressed as the Mueller matrix

$$
\mathbf{M}_{r o t}(\theta)=\left[\begin{array}{cccc}
1 & 0 & 0 & 0 \\
0 & \cos 2 \theta & \sin 2 \theta & 0 \\
0 & -\sin 2 \theta & \cos 2 \theta & 0 \\
0 & 0 & 0 & 1
\end{array}\right] .
$$

Rotation in the Mueller calculus is described in the same manner as in the Jones calculus. Equation (B.3) accounts for rotation in the Mueller calculus, but with the Jones matrices replaced by the corresponding Mueller matrices.

In the Mueller calculus, a waveplate with its fast axis parallel to the $x$-axis is given by

$$
\mathbf{M}_{w}(\delta)=\left[\begin{array}{cccc}
1 & 0 & 0 & 0 \\
0 & 0 & 0 & 0 \\
0 & 0 & \cos \delta & -\sin \delta \\
0 & 0 & \sin \delta & \cos \delta
\end{array}\right],
$$


where $\delta$ is the phase shift between the $x$ - and $y$-components of the incident beam. When oriented at an angle $\theta$, the waveplate Mueller matrix takes the form

$$
\mathbf{M}_{w}(\theta, \delta)=\left[\begin{array}{cccc}
1 & 0 & 0 & 0 \\
0 & \cos ^{2} 2 \theta+\cos \delta \sin ^{2} 2 \theta & (1-\cos \delta) \sin 2 \theta \cos 2 \theta & -\sin \delta \sin 2 \theta \\
0 & (1-\cos \delta) \sin 2 \theta \cos 2 \theta & \sin ^{2} 2 \theta+\cos \delta \cos ^{2} 2 \theta & \sin \delta \cos 2 \theta \\
0 & \sin \delta \sin 2 \theta & -\sin \delta \cos 2 \theta & \cos \delta
\end{array}\right] .
$$

The Mueller matrix for a linear polarizer is

$$
\mathbf{M}_{p}\left(k_{1}, k_{2}\right)=\frac{1}{2}\left[\begin{array}{cccc}
k_{1}^{2}+k_{2}^{2} & k_{1}^{2}-k_{2}^{2} & 0 & 0 \\
k_{1}^{2}-k_{2}^{2} & k_{1}^{2}+k_{2}^{2} & 0 & 0 \\
0 & 0 & 2 k_{1} k_{2} & 0 \\
0 & 0 & 0 & 2 k_{1} k_{2}
\end{array}\right] .
$$

When this element is at an angle $\theta$ relative to the $x$-axis, its Mueller matrix becomes

$$
\mathbf{M}_{p}\left(k_{1}, k_{2}, \theta\right)=\frac{1}{2}\left[\begin{array}{cccc}
p & q \cos 2 \theta & q \sin 2 \theta & 0 \\
q \cos 2 \theta & p \cos ^{2} 2 \theta+2 r \sin ^{2} 2 \theta & s(p-r) & 0 \\
q \sin 2 \theta & s(p-r) & p \sin ^{2} 2 \theta+2 r \cos ^{2} 2 \theta & 0 \\
0 & 0 & 0 & 2 r
\end{array}\right],
$$


where

$$
\begin{aligned}
& p=k_{1}^{2}+k_{2}^{2}, \\
& q=k_{1}^{2}-k_{2}^{2}, \\
& r=k_{1} k_{2}, \\
& s=\cos 2 \theta \sin 2 \theta .
\end{aligned}
$$

For an ideal linear polarizer, $k_{1}=1$ and $k_{2}=0$, and the Mueller matrix takes the form $[19,40]$

$$
\mathbf{M}_{p}(\theta)=\frac{1}{2}\left[\begin{array}{cccc}
1 & \cos 2 \theta & \sin 2 \theta & 0 \\
\cos 2 \theta & \cos ^{2} 2 \theta & \cos 2 \theta \sin 2 \theta & 0 \\
\sin 2 \theta & \cos 2 \theta \sin 2 \theta & \sin ^{2} 2 \theta & 0 \\
0 & 0 & 0 & 0
\end{array}\right] .
$$

All of the aforementioned Mueller matrices represent optical components. However, if a particular medium exhibits properties similar to an optical component, then the component Mueller matrices can be used for representing the medium. For example, a medium can be modeled as a waveplate with a parameterization in terms of retardance and fast axis orientation. 


\section{Appendix C Singular value decomposition}

The singular value decomposition (SVD) is a matrix factorization method often used for solving ill-conditioned inverse problems. This technique determines a total leastsquares solution, and accounts for errors in the independent and dependent variables. Additionally, the SVD can be used to investigate the conditioning of a system of linear equations, where the conditioning refers to how the system propagates errors. The ensuing discourse concentrates on the derivation and implementation of the SVD.

Singular value decomposition factors any $m \times n$ matrix $\mathbf{A}$ into two orthonormal matrices and one diagonal matrix. In other words, the SVD determines two orthonormal bases that diagonalize $\mathbf{A}$ [105]. Consider a real matrix $\mathbf{A}$ of dimension $m \times n$. From this, the matrix $\mathbf{A}^{T} \mathbf{A}$ can be created and is represented by $\mathbf{M}$,

$$
\mathbf{M}=\mathbf{A}^{T} \mathbf{A},
$$

where $\mathbf{M}$ is $n \times n$. Because $\mathbf{M}$ is real and symmetric, it can be diagonalized using a 
similarity transform,

$$
\mathbf{V}^{-1} \mathbf{M V}=\boldsymbol{\Lambda}=\left[\begin{array}{lll}
\lambda_{1} & & 0 \\
& \ddots & \\
0 & & \lambda_{n}
\end{array}\right]
$$

where $\mathbf{V}$ contains the eigenvectors of $\mathbf{M}$, inserted column-wise, and $\boldsymbol{\Lambda}$ is a diagonal matrix containing the eigenvalues $\left(\lambda_{1}, \lambda_{2}, \ldots \lambda_{n}\right)$ of $\mathbf{M}$ [105]. Additionally, since the eigenvectors of a symmetric matrix are orthogonal, the columns of $\mathbf{V}$ constitute a basis set. The matrix $\mathbf{V}$ is also said to be unitary, meaning its transpose is equal to its inverse, $\mathbf{V}^{T}=\mathbf{V}^{-1}$. Thus, the column vectors in $\mathbf{V}$ form an orthonormal basis set.

Now create the matrix $\mathbf{A} \mathbf{A}^{T}$ from $\mathbf{A}$;

$$
\mathbf{P}=\mathbf{A} \mathbf{A}^{T}
$$

where $\mathbf{P}$ is $m \times m$. Once again, $\mathbf{P}$ is real and symmetric, but now with $m$ linearly independent eigenvectors. We see that

$$
\mathbf{P U}=\mathbf{U} \Lambda
$$

where $\mathbf{U}$ contains the eigenvectors from $\mathbf{P}, \boldsymbol{\Lambda}$ is the eigenvalue matrix containing the eigenvalues of $\mathbf{P}$ (which are the same eigenvalues as $\mathbf{M}$ ), and $\mathbf{U}$ is unitary, $\mathbf{U}^{T}=\mathbf{U}^{-1}$. 
It can be seen that $\mathbf{P}$ is diagonalized by $\mathbf{U}[105]$,

$$
\mathbf{U}^{T} \mathbf{P} \mathbf{U}=\boldsymbol{\Lambda}=\left[\begin{array}{lll}
\lambda_{1} & & 0 \\
& \ddots & \\
& & \lambda_{m}
\end{array}\right]
$$

where $\lambda_{1} \ldots \lambda_{m}$ are the eigenvalues of $\mathbf{P}$, and the columns of $\mathbf{U}$ constitute an orthonormal basis set.

Now consider a new basis set containing the eigenvectors $\mathbf{u}_{k}$, described by

$$
\mathbf{u}_{k}=\frac{\mathbf{A} \mathbf{v}_{k}}{\sqrt{\lambda_{k}}}
$$

This basis set is also orthonormal because $\mathbf{U}$ satisfies the relationship [105]

$$
\mathbf{U}^{T} \mathbf{U}=\mathbf{u}_{i}^{T} \mathbf{u}_{j}=\left(\frac{\mathbf{A} \mathbf{v}_{i}}{\sqrt{\lambda_{i}}}\right)^{T} \frac{\mathbf{A} \mathbf{v}_{j}}{\sqrt{\lambda_{j}}}=\frac{\mathbf{v}_{i}^{T} \mathbf{A}^{T} \mathbf{A} \mathbf{v}_{j}}{\sqrt{\lambda_{i}} \sqrt{\lambda_{j}}}=\frac{\mathbf{v}_{i}^{T} \mathbf{M} \mathbf{v}_{j}}{\sqrt{\lambda_{i}} \sqrt{\lambda_{j}}}=\frac{\boldsymbol{\delta}_{i, j} \lambda_{j}}{\sqrt{\lambda_{i}} \sqrt{\lambda_{j}}}=\boldsymbol{\delta}_{i, j}
$$

Additionally, because $\mathbf{A}^{T} \mathbf{A}$ and $\mathbf{A} \mathbf{A}^{T}$ are both real, symmetric matrices, they are also positive semi-definite with non-negative eigenvalues [105]. Thus, the eigenvalues can be used to define $\sigma_{k}$, the singular values of $\mathbf{A}$,

$$
\sigma_{k}=\sqrt{\lambda_{k}}
$$


With this definition, equation (C.6) can be written [105]

$$
\mathbf{A v}_{k}=\mathbf{u}_{k} \sigma_{k}
$$

This can be expressed in matrix notation,

$$
\mathbf{A}\left[\begin{array}{c}
\mathbf{v}_{1} \\
\mathbf{v}_{2} \\
\vdots \\
\mathbf{v}_{n}
\end{array}\right]=\left[\begin{array}{c}
\mathbf{u}_{1} \\
\mathbf{u}_{2} \\
\vdots \\
\mathbf{u}_{m}
\end{array}\right]\left[\begin{array}{llll}
\sigma_{1} & & & 0 \\
& & & \\
& & \ddots & \\
0 & & & \sigma_{p}
\end{array}\right]
$$

where $p=\min (n, m)$. Finally, the SVD of $\mathbf{A}$ is given by

$$
\mathbf{A}=\mathbf{U} \boldsymbol{\Sigma} \mathbf{V}^{T},
$$

where $\mathbf{U}$ and $\mathbf{V}$ are orthonormal matrices with sizes $m \times m$ and $n \times n$, respectively. The matrix $\boldsymbol{\Sigma}$ is an $m \times n$ diagonal matrix with the singular values of $\mathbf{A}$ as its elements, typically arranged in decreasing order.

Rearranging the previous equation gives

$$
\boldsymbol{\Sigma}=\mathbf{U}^{T} \mathbf{A V} .
$$


It follows immediately from the orthonormality of $\mathbf{U}$ and $\mathbf{V}$ that

$$
\boldsymbol{\Sigma} \boldsymbol{\Sigma}^{T}=\left(\mathbf{U}^{T} \mathbf{A V}\right)\left(\mathbf{U}^{T} \mathbf{A V}\right)^{T}=\mathbf{U}^{T} \mathbf{A} \mathbf{V} \mathbf{V}^{T} \mathbf{A}^{T} \mathbf{U}=\mathbf{U}^{T} \mathbf{A} \mathbf{A}^{T} \mathbf{U}
$$

and

$$
\boldsymbol{\Sigma}^{T} \boldsymbol{\Sigma}=\left(\mathbf{U}^{T} \mathbf{A} \mathbf{V}\right)^{T}\left(\mathbf{U}^{T} \mathbf{A V}\right)=\mathbf{V}^{T} \mathbf{A}^{T} \mathbf{A} \mathbf{U} \mathbf{U}^{T} \mathbf{A V}=\mathbf{V}^{T} \mathbf{A}^{T} \mathbf{A} \mathbf{V}
$$

The diagonal matrices $\boldsymbol{\Sigma} \boldsymbol{\Sigma}^{T}$ and $\boldsymbol{\Sigma}^{T} \boldsymbol{\Sigma}$ share the same non-zero eigenvalues, which are the eigenvalues of $\mathbf{A} \mathbf{A}^{T}$ and $\mathbf{A}^{T} \mathbf{A}$, respectively. From this, we see that $\mathbf{U}$ and $\mathbf{V}$ diagonalize $\mathbf{A} \mathbf{A}^{T}$ and $\mathbf{A}^{T} \mathbf{A}$, respectively, through a similarity transform. Also, the eigenvectors of $\mathbf{A A}^{T}$ are the column vectors of $\mathbf{U}$. The same relationship holds for $\mathbf{A}^{T} \mathbf{A}$ and $\mathbf{V}[105]$.

With $\mathbf{U}, \mathbf{V}$, and $\boldsymbol{\Sigma}$ established, the pseudoinverse $\mathbf{A}^{+}$can be calculated,

$$
\mathbf{A}^{+}=\left(\mathbf{U} \boldsymbol{\Sigma} \mathbf{V}^{T}\right)^{-1},
$$

which becomes

$$
\mathbf{A}^{+}=\mathbf{V} \boldsymbol{\Sigma}^{+} \mathbf{U}^{T}=\mathbf{V} \operatorname{diag}\left(1 / \sigma_{1}, \ldots, 1 / \sigma_{p}, 0, \ldots, 0\right) \mathbf{U}^{T},
$$

where $\mathbf{A}^{+}$is the pseudoinverse of $\mathbf{A}$ and is of dimension $n \times m, \mathbf{V}$ is $n \times n, \boldsymbol{\Sigma}^{+}$is the 
pseudoinverse of $\boldsymbol{\Sigma}$ and is of dimension $n \times m, \mathbf{U}^{T}$ is $m \times m$, and $p=\min (n, m)$.

Using the SVD for finding the pseudoinverse provides the option of truncation. Truncating the pseudoinverse implements a total-least squares solution that takes into account errors in the dependent and independent variables. This is accomplished by setting small, non-zero singular values to zero. These values represent noise and are outside of the system dimension. Thus, truncation ensures the desired dimensionality of the pseudoinverse, and it also minimizes the condition number of the given matrix [93].

The condition number is a matrix property that describes its nearness to singularity, and also how easily one can "invert" a series of equations. A minimum condition number indicates that the linear independence of rows and columns is maximized. The condition number also represents the sensitivity of a linear system of equations to errors, and how those errors propagate [105]. For example, in the linear equation $\mathbf{A x}=\mathbf{b}$, the condition number of $\mathbf{A}$ relates how much errors in $\mathbf{A}$ and/or $\mathbf{b}$ are magnified in the solution vector $\mathbf{x}$. The matrix $\mathbf{A}$ is said to be ill-conditioned (high condition number) if a small perturbation in $\mathbf{A}$ or $\mathbf{b}$ results in a large change in $\mathbf{x}$. Matrices with low condition numbers are said to be well-conditioned [105].

Finally, the SVD is a valuable tool for numerical analysis and signal processing. It can be used for overdetermined and underdetermined least-squares problems, investigating the conditioning of ill-conditioned systems, computing pseudoinverses, 
and matrix approximation. Specific applications of the SVD include spectral analysis, principal component analysis, image reconstruction, and noise reduction [105]. 


\section{Appendix D Lyot filter}

The LCTF used in the system configuration is based on the Lyot filter, which is a type of static bandpass optical filter. Lyot filters transmit a specific wavelength spectrum based on interference effects produced by a series of polarizing optics. The remainder of this appendix focuses on the construction and functionality of Lyot filters.

The fundamental optical component of a Lyot filter is a birefringent element sandwiched between a pair of polarizers, shown in the schematic of Fig. D.1. In general, these components make up a single stage in the Lyot filter. A single stage functions by employing an entrance polarizer for creating a linear polarization state incident on the ensuing birefringent element (often quartz or calcite). Next, the birefringent element, oriented at $45^{\circ}$ relative to the transmission axis of the entrance and exit polarizers, resolves the incident polarization state into orthogonal polarization components. The exit polarizer recombines the components, thus producing interfer-

ence effects analogous to a Michelson interferometer [141]. This creates a transmission 


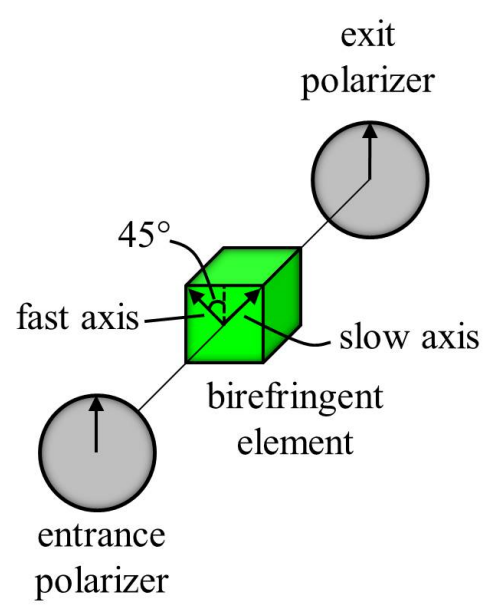

Figure D.1: Schematic representation of the fundamental components in a Lyot filter. These components typically make up a single stage inside the filter.

profile for a single stage that is described by $[21,141]$,

$$
T=\frac{1}{2} \cos ^{2}\left(\frac{2 \pi \Delta n d}{\lambda}\right),
$$

where $\Delta n$ is the birefringence, $d$ is the birefringent element thickness, and $\lambda$ is the wavelength of the incident light.

For a birefringent element with thickness $d$, the transmission profile is periodic, shown in Fig. D.2a. When the element thickness doubles to $2 d$, the transmission profile periodicity decreases, also shown in Fig. D.2a. Now consider a system composed of two stages, where the stages have birefringent elements with thicknesses $d$ and 2d. The overall transmission (see Fig. D.2b) is the product of the two individual transmission spectra from Fig. D.2a. Therefore, adding stages with specific element thicknesses provides a method for manipulating the overall system transmission. 


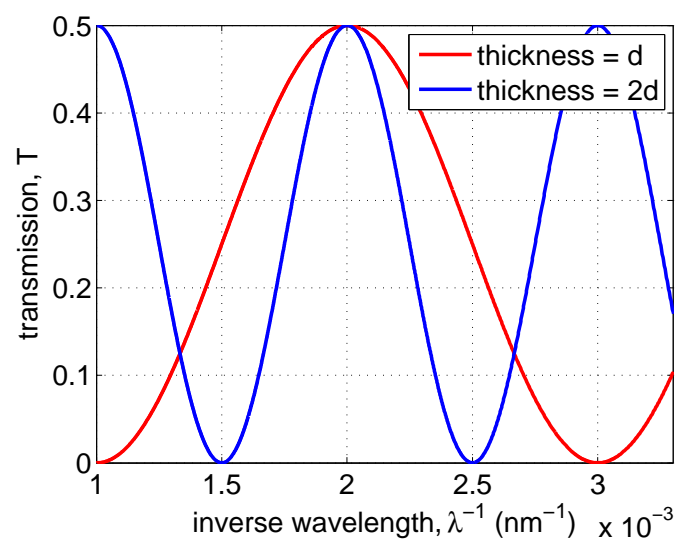

(a)

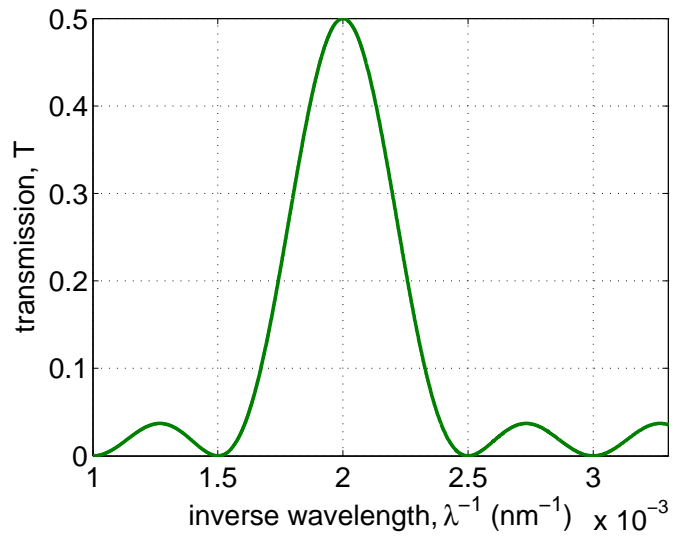

(b)

Figure D.2: a) Single-stage transmission profiles for two different birefringent element thicknesses, b) Two-stage transmission profile.

Lyot filters consist of a series of stages, shown in Fig. D.3, where each successive stage uses the exit polarizer of the previous stage as its entrance polarizer. The birefringent element thickness in each stage, however, doubles in size relative to the previous stage. Thus, the thickness $d$ of each element is given by [21]

$$
d_{i+1}=2 d_{i}=2^{i} d_{1}
$$

where $d_{1}$ is the thickness of the first birefringent element. Now recall equation (D.1), the transmission for a single stage. This equation can be written in terms of the retardance for the $i^{\text {th }}$ birefringent element $\Gamma_{i}$,

$$
T=\frac{1}{2} \cos ^{2}\left(\frac{2 \pi \Delta n d_{i}}{\lambda}\right)=\frac{1}{2} \cos ^{2}\left(\frac{\Gamma_{i}}{2}\right)
$$




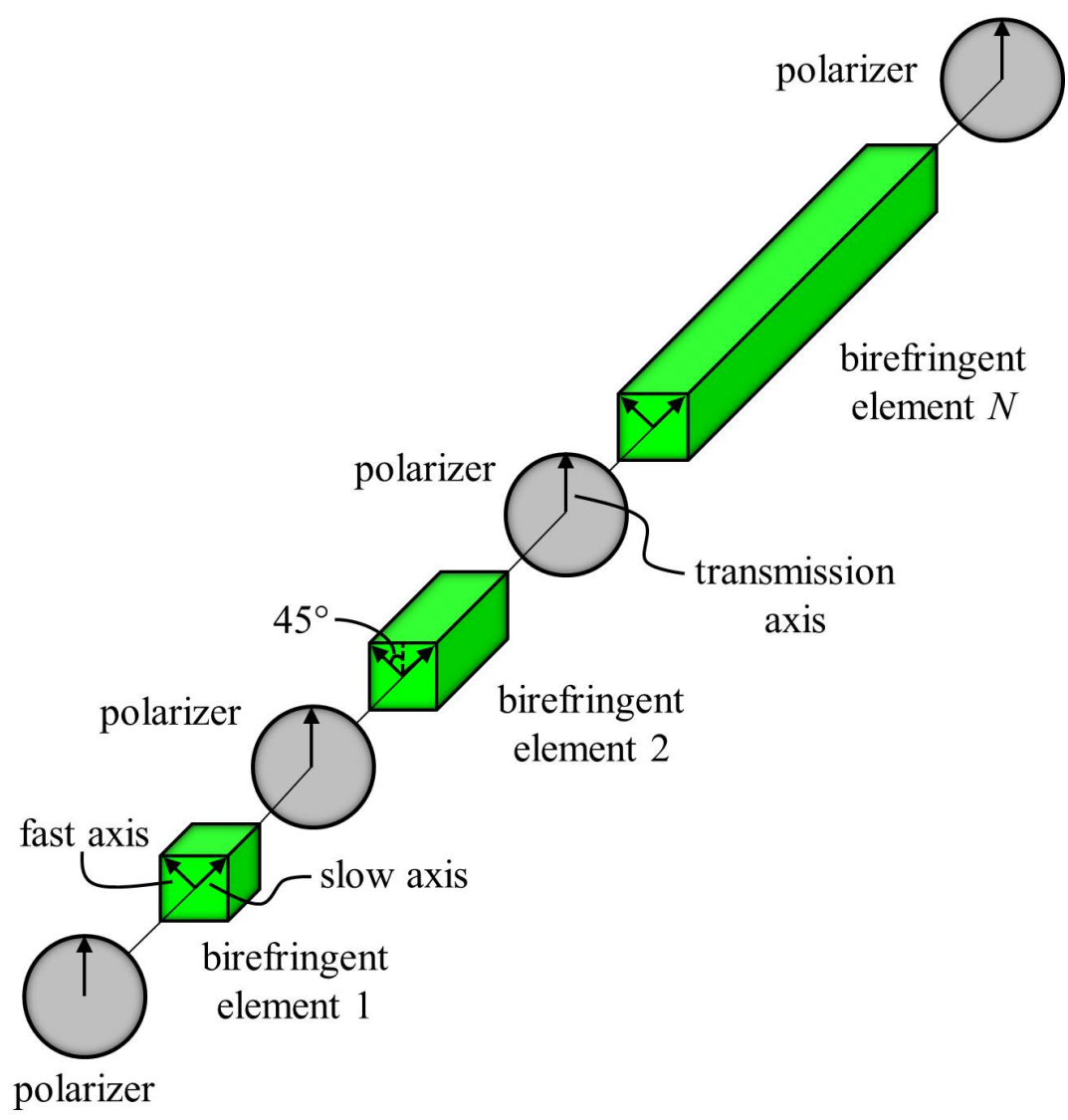

Figure D.3: Schematic representation of an $N$ stage Lyot filter.

For a series of $N$ stages, the overall transmission profile for a Lyot filter is $[21,141]$

$$
T=\frac{1}{2} \prod_{i=1}^{N} \cos ^{2}\left(\frac{2^{i-1} \Gamma_{1}}{2}\right)=\frac{1}{2}\left[\frac{\sin \left(2^{N-1} \Gamma_{1}\right)}{2^{N} \sin \left(\Gamma_{1} / 2\right)}\right]^{2} .
$$

Figure D.4 shows the overall transmission profile for an $N=5$ stage Lyot filter. According to this figure, the Lyot filter produces a transmission spectrum that exhibits a central peak at a given wavelength and almost negligible transmission at others. Increasing the number of stages has a two-fold effect, as it decreases the width of the central peak passband and suppresses transmission at other wavelengths. These 


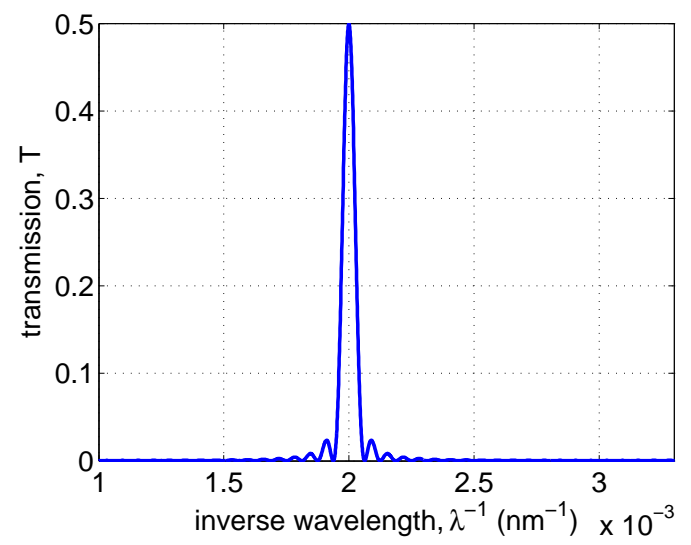

Figure D.4: Transmission profile for an $N=5$ stage Lyot filter.

results show the functionality of the conventional Lyot filter. This functionality has been modified such that the filter now provides spectral tuning ability.

Spectral tuning ability in Lyot filters can be implemented with three different techniques. The first technique requires adjustment of the birefringent optical path length by either temperature control or mechanical movement of a birefringent plate. For the mechanical movement technique, a pair of wedge-shaped plates is used, where one plate slides across the other. This movement changes the physical thickness and thus the birefringence. A second method introduces additional birefringent elements (i.e., half wave plates and quarter wave plates), and then requires the rotation of either the exit polarizer or one of the wave plates. The final technique, the electrooptical method, utilizes liquid crystal cells. For this technique, a liquid crystal cell and a fixed retarder replace the original birefringent element of each stage $[113,141]$.

Cambridge Reasearch and Instrumentation (CRI) employs the electro-optical method for manufacturing the LCTF used in this work [112,113]. The CRI design uses 
six tunable birefringent stages [112], which results in a spectral range of $\lambda=400-720$ $\mathrm{nm}$ with a $7 \mathrm{~nm}$ bandwidth and a switching time of 50 milliseconds. 


\section{Appendix E Coherence functions}

Coherence means correlation. If two fields are correlated, then they are said to be coherent. Coherence is the correlation between fields at two different points in time or space. Describing the correlation in this manner distinguishes two types of coherence, temporal and spatial. This description is based on the statistical properties of the waveform fluctuations in time and space. Temporal coherence represents the ability of a field to interfere with a time-delayed version of itself. This can also be thought of as the correlation between two points in space separated along the direction of propagation. Spatial coherence describes the ability of a field to interfere with a spatially shifted version of itself. Another interpretation of spatial coherence is the correlation between two points in a plane transverse to the propagation direction.

Many metrics parameterize temporal and spatial coherence, most commonly as second-order averages called coherence functions. Second-order coherence phenomena are a function of either position and time, or position and frequency. The rest of this appendix describes temporal and spatial coherence, and expresses them in terms of the aforementioned second-order averages. 


\section{Temporal coherence}

Temporal coherence can be described in terms of a quasi-monochromatic field. A real, quasi-monochromatic signal (i.e., a field disturbance) is represented as [20]

$$
u^{(r)}(t)=A(t) \cos \left(2 \pi \nu_{0} t-\phi(t)\right),
$$

where $A(t)$ is the time-dependent field amplitude (which varies slowly with respect to $\left.\nu_{0}\right), \nu_{0}$ is the frequency, and $\phi(t)$ is a time-dependent phase. The analytic representation of this is

$$
u(t)=A(t) e^{i \phi(t)} e^{-i 2 \pi \nu_{0} t},
$$

where $A(t) e^{i \phi(t)}$ is the time-varying phasor, or complex envelope function. This representation also can be a function of position $\mathbf{r}$, where $\mathbf{r}=(x, y, z)$ is a point in space. Quasi-monochromatic light is defined such that its effective bandwidth $\Delta \nu$ is much smaller than its center frequency $\nu_{0}, \Delta \nu / \nu_{0} \ll 1[20]$.

Because the signal has a finite bandwidth $\Delta \nu$, the amplitude and phase of the envelope function are slowly varying in time with respect to $e^{-i 2 \pi \nu_{0} t}$. In other words, the bandwidth determines the rate at which the phasor changes. For a given time $\tau$, the envelope remains relatively constant provided that $\tau \sim 1 / \Delta \nu[20]$. This suggests a high degree of correlation between two waves delayed by a time $\tau$. Therefore, the 
bandwidth serves as an indicator of the coherence time $\tau_{c}$ of two fields,

$$
\tau_{c}=\frac{1}{\Delta \nu} .
$$

The coherence length is the distance the wave travels within the coherence time,

$$
l_{c}=c \tau_{c} .
$$

The coherence length can also be expressed as

$$
l_{c}=\frac{\overline{\lambda^{2}}}{\Delta \lambda},
$$

where $\bar{\lambda}=c / \bar{\nu}$ is the mean wavelength for the field, and $\Delta \lambda$ is the optical bandwidth [142]. As can be seen from equation (E.5), a narrow bandwidth results in a large coherence length. Thus, temporal coherence refers to the spectral purity of the source, and it is not an intrinsic property of the source. It is possible to introduce a degree of temporal coherence by using narrowband wavelength filters.

\section{Spatial coherence}

While temporal coherence relies on spectral purity, spatial coherence depends on the apparent physical size of the source. Spatial coherence refers to the degree to which a source looks like a geometrical point. A simple method for relating source 


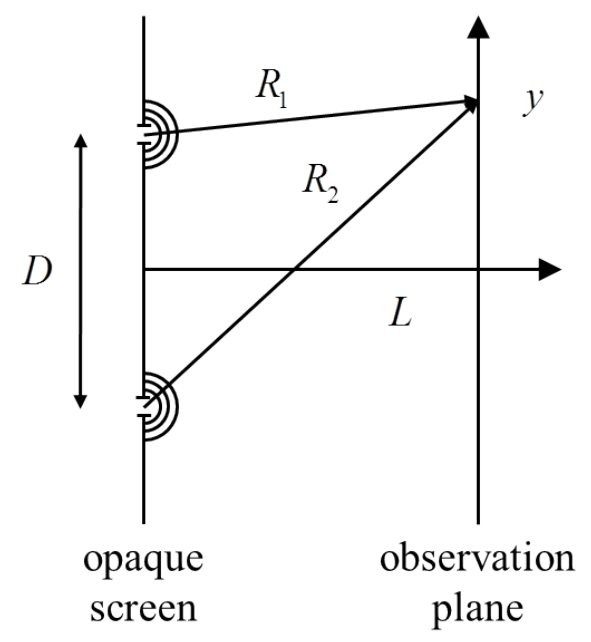

Figure E.1: Schematic of Young's interference experiment.

size and spatial coherence uses Young's interference experiment (see Fig. E.1). In this experiment, an opaque screen with two pinholes is illuminated with a quasimonochromatic source. The two pinholes are treated as equivalent secondary sources.

The field $U$ in the observation plane at point $y$ due to the point sources is [143]

$$
U(y, L)=\frac{e^{i k R_{1}}}{R_{1}}+\frac{e^{i k R_{2}}}{R_{2}},
$$

where $k=2 \pi / \lambda$ is the wavenumber, the exponential terms represent the spherical wavefronts emanating from each pinhole, and the distances are

$$
\begin{aligned}
& R_{1}=\sqrt{(y-D / 2)^{2}+L^{2}} \\
& R_{2}=\sqrt{(y+D / 2)^{2}+L^{2}} .
\end{aligned}
$$


Under the paraxial approximation, the observation distance $L$ is much greater than $D$ and $y$. Using this approximation, along with the first two terms of the Taylor series expansion, allows one to write

$$
\begin{aligned}
& R_{1} \approx L+\frac{(y-D / 2)^{2}}{2 L} \\
& R_{2} \approx L+\frac{(y+D / 2)^{2}}{2 L} .
\end{aligned}
$$

A final approximation gives

$$
R_{1} \approx R_{2} \approx L
$$

The corresponding intensity for these fields at point $y$ is [143]

$$
\begin{aligned}
I(y, L) & =|U(y, L)|^{2}=\left|\frac{e^{i k R_{1}}}{L}+\frac{e^{i k R_{2}}}{L}\right|^{2} \\
& =\frac{1}{L^{2}}\left[2+2 \cos k\left(R_{1}-R_{2}\right)\right] \\
& \approx \frac{1}{L^{2}}\left[1+\cos \left(\frac{k y D}{L}\right)\right]
\end{aligned}
$$

According to the last equation above, a fringe pattern will be visible in the observation plane with spatial frequency

$$
T=\frac{\lambda L}{D} .
$$

The equations above assume that the slits are very small compared to the wavelength of light. Thus, the slits behave like point sources, and they produce interference 
effects in the observation plane.

Now assume there is a second, identical quasi-monochromatic source behind the opaque screen. The second source also will produce fringes of period $T=\lambda L / D$ in the observation plane. If the second source is displaced at an angle $\alpha$ relative to the original quasi-monochromatic source, the fringe pattern of the second source will be displaced accordingly. For small angular displacements, the total fringe pattern will have high visibility. However, if the angular displacement is large enough, it will cause the peak of one fringe pattern to correspond to the minimum of the other, reducing the visibility. The threshold angular displacement that causes this specific overlap in the fringe pattern is the case where the displacement $\alpha L$ is equal to half of the fringe period,

$$
\begin{gathered}
\alpha L=\frac{\lambda L}{2 D}, \\
\alpha=\frac{\lambda}{2 D} .
\end{gathered}
$$

Equation (E.12) also shows that the distance $D$ between the pinholes impacts fringe visibility. The fringes will have good visibility if the fields in the pinholes are coherent. Thus, there is a distance $D$ where fringes of high visibility can be observed,

$$
D<\frac{\lambda}{2 \alpha}
$$


This can be thought of as the coherence diameter within the plane of the apertures,

$$
D_{c}<\frac{\lambda}{2 \alpha},
$$

where $2 \alpha$ is the apparent angular subtense of the source. Decreasing the angular subtense, or apparent size of the source, increases the coherence diameter. Spatial coherence is not an intrinsic property of a field, but a degree of spatial coherence can be introduced in a field by limiting the apparent size of the source.

\section{Coherence as a function of space and time}

Temporal and spatial coherence are described in terms of the mutual coherence function, which is a correlation function as a function of space and time. The mutual coherence function is defined as

$$
\Gamma\left(\mathbf{r}_{1}, \mathbf{r}_{2}, \tau\right)=\left\langle u\left(\mathbf{r}_{1}, t+\tau\right) u^{*}\left(\mathbf{r}_{2}, t\right)\right\rangle,
$$

where the brackets denote an ensemble average, $*$ denotes the complex conjugate, and $\tau=t_{2}-t_{1}$. Equation (E.15) depends on the time difference $\tau$ when the field fluctuations are stationary, at least in the wide sense [144]. This expression is a crosscorrelation function at positions $\mathbf{r}_{1}$ and $\mathbf{r}_{2}$ as a function of time delay $\tau$, and describes the spatial correlation at a given $\tau[20]$.

The normalized version of the mutual coherence function is the complex degree 
of coherence,

$$
\gamma\left(\mathbf{r}_{1}, \mathbf{r}_{2}, \tau\right)=\frac{\left\langle u\left(\mathbf{r}_{1}, t+\tau\right) u^{*}\left(\mathbf{r}_{2}, t\right)\right\rangle}{\left[\left\langle u\left(\mathbf{r}_{1}, t\right) u^{*}\left(\mathbf{r}_{1}, t\right)\right\rangle\left\langle u\left(\mathbf{r}_{2}, t\right) u^{*}\left(\mathbf{r}_{2}, t\right)\right\rangle\right]^{\frac{1}{2}}}=\frac{\Gamma_{12}(\tau)}{\left[\Gamma_{11}(0) \Gamma_{22}(0)\right]^{\frac{1}{2}}},
$$

which provides a measure of the statistical similarity between the fluctuations at $\mathbf{r}_{1}$ and $\mathbf{r}_{2}$ for the given time delay [20]. The absolute value of the complex degree of coherence has values such that

$$
\left|\gamma\left(\mathbf{r}_{1}, \mathbf{r}_{2}, \tau\right)\right| \leq 1
$$

Typically, temporal and spatial coherence cannot be treated independently, due to the differential equations governing the propagation of mutual coherence [20]. However, when concentrating on spatial coherence, it is often convenient to set the time delay $\tau$ in equation (E.15) equal to zero. This is a commonly accepted practice when symmetry exists in the experimental set-up or when one is dealing with imaging near the axis of a centered optical system [127]. The mutual coherence function at $\tau=0$ is defined as the mutual intensity,

$$
J\left(\mathbf{r}_{1}, \mathbf{r}_{2}\right)=\Gamma\left(\mathbf{r}_{1}, \mathbf{r}_{2}, 0\right) .
$$


The normalized version of the mutual intensity $J$ is the complex coherence factor $j$,

$$
j\left(\mathbf{r}_{1}, \mathbf{r}_{2}\right)=\gamma\left(\mathbf{r}_{1}, \mathbf{r}_{2}, 0\right)=\frac{J\left(\mathbf{r}_{1}, \mathbf{r}_{2}\right)}{\left[J\left(\mathbf{r}_{1}, \mathbf{r}_{1}\right) J\left(\mathbf{r}_{2}, \mathbf{r}_{2}\right)\right]^{\frac{1}{2}}}=\frac{J\left(\mathbf{r}_{1}, \mathbf{r}_{2}\right)}{\left[I\left(\mathbf{r}_{1}\right) I\left(\mathbf{r}_{2}\right)\right]^{\frac{1}{2}}},
$$

where $I$ represents the intensity at the given point and $j$ has values of

$$
\left|j\left(\mathbf{r}_{1}, \mathbf{r}_{2}\right)\right| \leq 1
$$

\section{Coherence as a function of space and frequency}

The mutual coherence function forms a Fourier transform pair with the cross-spectral density function $W\left(\mathbf{r}_{1}, \mathbf{r}_{2}, \nu\right)$. The function $W\left(\mathbf{r}_{1}, \mathbf{r}_{2}, \nu\right)$ describes the spatial correlation at two points $\mathbf{r}_{1}$ and $\mathbf{r}_{2}$ for a given frequency $\nu$ and is [126]

$$
W\left(\mathbf{r}_{1}, \mathbf{r}_{2}, \nu\right)=\frac{1}{2 \pi} \int_{-\infty}^{\infty} \Gamma\left(\mathbf{r}_{1}, \mathbf{r}_{2}, \tau\right) e^{i \nu \tau} \mathrm{d} \tau .
$$

The other half of the transform pair is

$$
\Gamma\left(\mathbf{r}_{1}, \mathbf{r}_{2}, \tau\right)=\int_{0}^{\infty} W\left(\mathbf{r}_{1}, \mathbf{r}_{2}, \nu\right) e^{-i \nu \tau} \mathrm{d} \nu .
$$

This relationship is known as the Wiener-Khintchine theorem [127].

The normalized version of the cross-spectral density function is the complex 
degree of spectral coherence,

$$
\mu\left(\mathbf{r}_{1}, \mathbf{r}_{2}, \nu\right)=\frac{W\left(\mathbf{r}_{1}, \mathbf{r}_{2}, \nu\right)}{\left[W\left(\mathbf{r}_{1}, \mathbf{r}_{1}, \nu\right) W\left(\mathbf{r}_{2}, \mathbf{r}_{2}, \nu\right)\right]^{\frac{1}{2}}},
$$

which is a measure of the degree of spatial coherence at a given frequency [126]. This parameter satisfies the inequality

$$
\left|\mu\left(\mathbf{r}_{1}, \mathbf{r}_{2}, \nu\right)\right| \leq 1
$$

In closing this section, various concepts and functions were introduced that serve as fundamental tools for studying coherence. The functions presented here also act as the foundation for an important propagation law called the van Cittert-Zernike theorem, which is discussed in Chapter 4. 


\section{Appendix F Köhler illumination}

Köhler illumination provides specific illumination characteristics by utilizing an optical configuration based on variable-diameter irises. This configuration controls the lateral extent and homogeneity of the illumination pattern, and the spatial coherence at the sample plane [114]. In terms of the optical layout, Köhler illumination consists of a series of lenses and variable-diameter irises, which are placed at specific positions along the optical axis. The subsequent discussion explains the configuration and concepts governing Köhler illumination.

Figure F.1 shows a schematic of the Köhler illumination configuration. In this configuration, a light source is placed in the back focal plane of lens 1 . In the front focal plane of lens 1 is a variable-diameter iris, which serves as the field stop. This field stop is also positioned in the back focal plane of lens 2. Next, the front focal plane of lens 2 contains a second variable-diameter iris, and this iris acts as the aperture stop. Additionally, the aperture stop location coincides with the back focal

plane of lens 3. Finally, the sample plane is in the front focal plane of lens 3. Because of the specific positions of the field and aperture stops, there are Fourier transform 


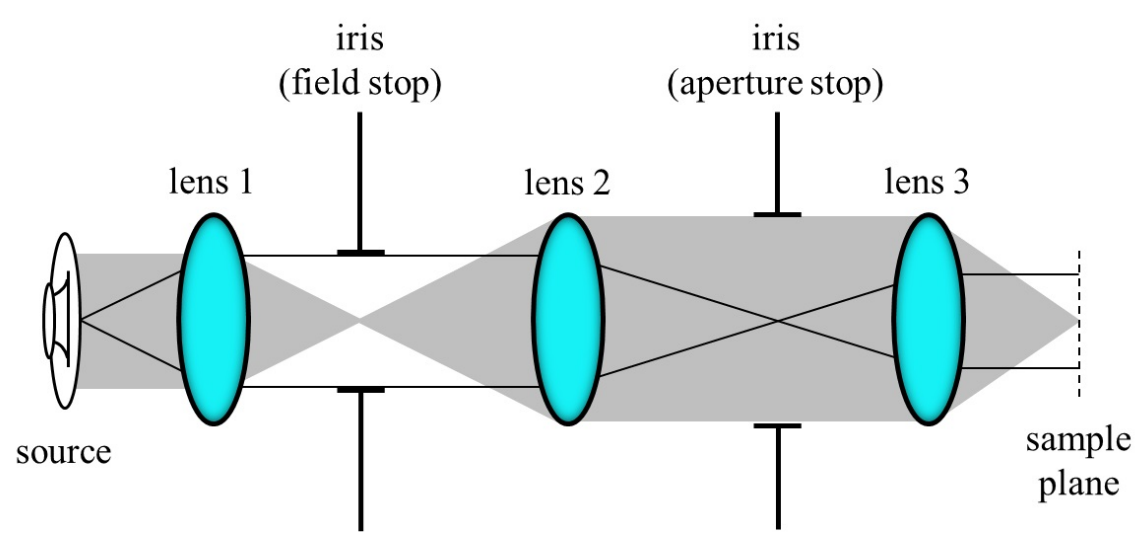

Figure F.1: Schematic of the Köhler illumination configuration.

relationships between certain planes, which will be discussed next.

The field and aperture stops have specific roles in the imaging system, as they dictate conjugate planes and control illumination characteristics. In terms of conjugate planes, two planes are conjugate if the intensity distribution of one plane is imaged to the other plane [120]. Conjugate planes arise in Köhler illumination because of both the Fourier transforming property of lenses and the spatial relationships between the lenses. For example, if an object is placed in the back focal plane of a lens, the Fourier transform (FT) (i.e., the 2D spatial frequency distribution) of the object appears in the front focal plane of the lens. Placing a second lens a focal length away from the FT plane produces an image of the object in the front focal plane of the second lens, making the object plane and image plane a conjugate pair. For the Köhler configuration, FT relationships exist between the source and the field stop, the field stop and the aperture stop, and the aperture stop and the sample plane (see Fig. F.2). Thus, the source plane is imaged to the aperture stop plane, making these 


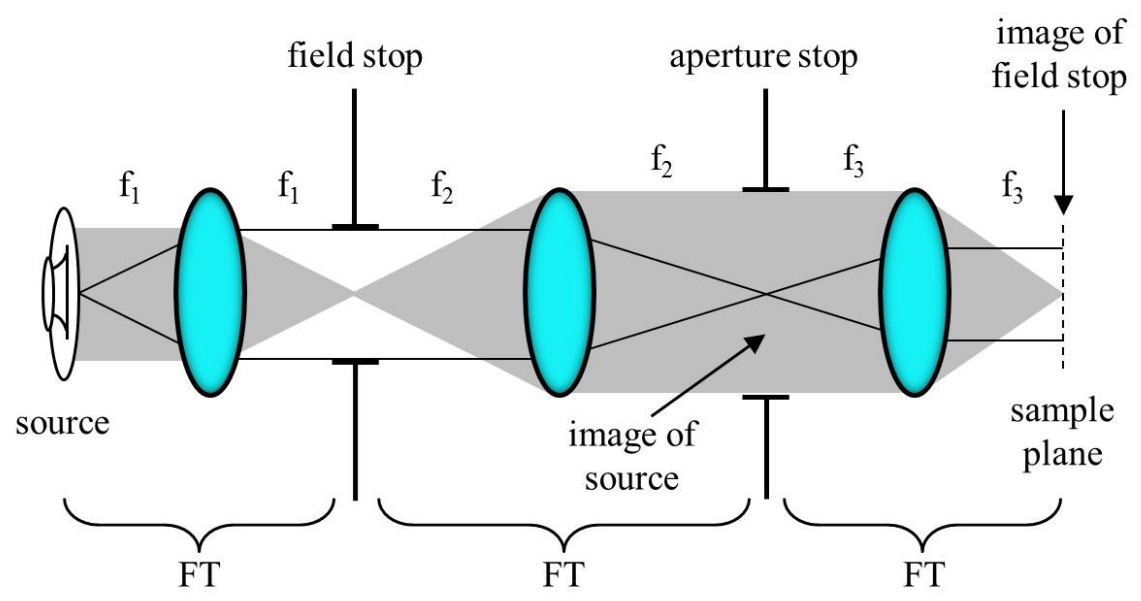

Figure F.2: Köhler illumination schematic showing the FT relationships.

two planes conjugate. Additionally, the field stop is imaged at the sample plane.

The field and aperture stops also control illumination characteristics. Because the field stop is imaged at the sample plane, it sets the illumination field. In other words, the field stop limits the field of view of the imaging system, and specifies the size of the illumination pattern at the sample plane. On the other hand, the aperture stop controls brightness and spatial coherence. Opening the aperture stop increases the brightness at the sample plane, and it also increases the angular extent of the source. Thus, the aperture stop specifies the size of the illumination cone (i.e., the spatial coherence) at the sample plane. Closing down the aperture stop provides more directional illumination (high spatial coherence), while opening the stop increases the cone angle and decreases the spatial coherence [114].

In summary, this appendix described the characteristics of Köhler illumination. The Köhler configuration provides optimal sample illumination by controlling the size 
of both the illumination pattern and the illumination cone at the sample plane. Thus, it is also possible to control illumination coherence. Furthermore, because the field stop is imaged to the sample plane, Köhler illumination delivers a uniform, structurefree pattern to the sample plane. 


\section{Appendix G Polarizer characterization}

Polarizer characterization quantifies the spectral dependence of the principal transmittances $\left(k_{1}^{2}\right.$ and $\left.k_{2}^{2}\right)$ of the element. Characterization involves making transmission measurements and performing a regression analysis for the two unknowns, $k_{1}^{2}$ and $k_{2}^{2}$. This appendix describes the entire procedure for polarizer characterization, which includes the measurement configuration and the relevant theory. The discussion begins with the measurement configuration, as it provides a reference point for the subsequent theoretical development.

\section{Measurement configuration}

Measurements for polarizer characterization consisted of illuminating a pair of identical polarizers with a broadband source, and measuring the orientation-dependent transmission with a spectrometer. A schematic of the measurement configuration is shown in Fig. G.1. In this configuration, a broadband light source was roughly collimated by a lens. Following the lens were the pair of polarizers to be characterized, an integrating sphere (Labsphere, 6-inch diameter), and an Ocean Optics QE65000 

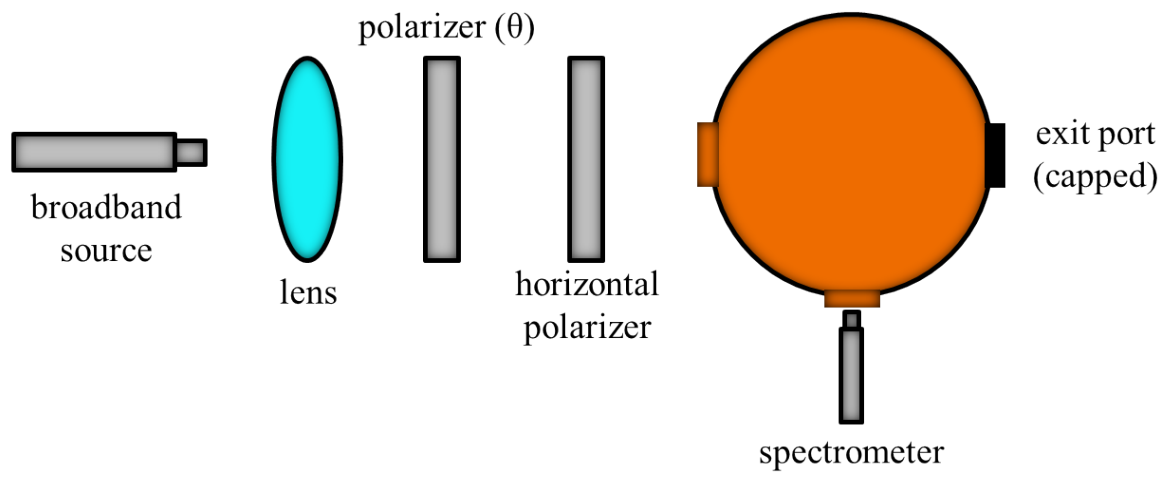

Figure G.1: Schematic of the measurement configuration for determining the polarizer principal transmittances.

spectrometer (16 bit). The polarizers are two Newport linear sheet polarizers (10LPVIS-B). Since the polarizers were identical, there were only two unknowns, $k_{1}^{2}$ and $k_{2}^{2}$, which apply to both polarizers.

The transmission measurements on the polarizer pair consisted of three individual intensity measurements. These three measurements were the intensity transmitted by the polarizers $I(\lambda)$, a background measurement with the light source blocked $I_{\mathrm{bg}}(\lambda)$, and a measurement without the components $I_{0}(\lambda)$. These were then related to the polarizer transmittance $T(\lambda)$ as follows,

$$
T(\lambda)=\frac{I(\lambda)-I_{\mathrm{bg}}(\lambda)}{I_{0}(\lambda)-I_{\mathrm{bg}}(\lambda)} .
$$

For the measurements, the first polarizer was rotated through an angle $\theta$ from $0^{\circ}-90^{\circ}$ in $10^{\circ}$ increments. The second polarizer was at a constant angle of $0^{\circ}$ with respect to the $x$-axis of the laboratory reference frame (i.e., horizontally polarized). 
Transmission measurements were made at each angle $\theta$.

\section{Parameterization with the Jones calculus}

In reference to Appendix B, the Jones matrix representing a linear polarizer $\mathbf{L}_{H}$ is,

$$
\mathbf{L}_{H}=\left[\begin{array}{ll}
k_{1} & 0 \\
0 & k_{2}
\end{array}\right]
$$

where $k_{1}$ and $k_{2}$ are the spectrally-dependent polarizer principal field transmittances (the wavelength dependence is dropped for simplicity). The Jones matrix for a linear polarizer at an angle $\theta$ with respect to the $x$-axis of the laboratory reference frame is

$$
\mathbf{L}_{\text {pol }}=\left[\begin{array}{cc}
k_{1} \cos ^{2} \theta+k_{2} \sin ^{2} \theta & k_{1} \cos \theta \sin \theta-k_{2} \cos \theta \sin \theta \\
k_{1} \cos \theta \sin \theta-k_{2} \cos \theta \sin \theta & k_{1} \sin ^{2} \theta+k_{2} \cos ^{2} \theta
\end{array}\right] .
$$

The composite Jones matrix for the two-polarizer system is found by multiplying equations (G.2) and (G.3). Applying the coherency matrix formalism to the composite Jones matrix consisting of $\mathbf{L}_{H}$ and $\mathbf{L}_{\text {pol }}$ results in the following expression for the transmitted intensity,

$$
T=\frac{c}{2}\left[k_{1}^{4} \cos ^{2} \theta+k_{2}^{4} \cos ^{2} \theta+2 k_{1}^{2} k_{2}^{2} \sin ^{2} \theta\right],
$$


where $c$ is an unknown scaling constant dependent on the measurement system.

Equation (G.4) has two unknowns, $k_{1}^{2}$ and $k_{2}^{2}$ (the parameter $c$ is ignored), thus the equation cannot be solved in the current form. A regression analysis is necessary, as this type of analysis is used for exploring the relationship between dependent and independent variables when unknown parameters are involved. Regression analysis allows for the estimation of independent parameters, such as $k_{1}^{2}$ and $k_{2}^{2}$. Additionally, because equation (G.4) contains the two parameters both separately and in product, the transmission expression must be diagonalized for the regression analysis. The next section describes details of the diagonalization and data processing.

\section{Similarity transformation}

A similarity transformation is a tool that is used for converting a relationship (i.e., equation (G.4)) into a form that is suitable for regression analysis. In equation (G.4), the independent variable is $\theta$, the dependent variable is $T$, and the unknown parameters are $k_{1}$ and $k_{2}$. The initial step for implementing a similarity transformation is to convert equation (G.4) to matrix notation. The matrix notation is as follows,

$$
\mathbf{2} \mathbf{T}=\mathbf{k}^{T} \mathbf{A} \mathbf{k}=\left[\begin{array}{ll}
k_{1}^{2} & k_{2}^{2}
\end{array}\right]\left[\begin{array}{ll}
\cos ^{2} \theta & \sin ^{2} \theta \\
\sin ^{2} \theta & \cos ^{2} \theta
\end{array}\right]\left[\begin{array}{l}
k_{1}^{2} \\
k_{2}^{2}
\end{array}\right],
$$

where the factor of two is from equation (G.4) and $\mathbf{A}$ is a transformation matrix representing the characteristics of the equation to be analyzed. 
With equation (G.5) established, a similarity transformation can now be performed. Matrix A is expressed in terms of its eigenvectors and eigenvalues,

$$
\mathrm{GA}=\Lambda \mathrm{G}
$$

where $\mathbf{G}$ is a matrix of eigenvectors, assembled column-wise, and $\boldsymbol{\Lambda}$ is a diagonal matrix with eigenvalues on the diagonal. The eigenvalues are determined by the equation

$$
\operatorname{det}(\mathbf{A}-\lambda \mathbf{I})=0
$$

where $\mathbf{A}$ was defined in equation (G.5), $\lambda$ are the eigenvalues, and $\mathbf{I}$ is the identity matrix. Solving the linear algebra to find the eigenvalues gives

$$
\boldsymbol{\Lambda}=\left[\begin{array}{cc}
1 & 0 \\
0 & \cos 2 \theta
\end{array}\right]
$$

Using equation (G.6) and the eigenvalues from equation (G.8), the transformation matrix $\mathbf{G}$ can be found,

$$
\mathbf{G}=\frac{1}{\sqrt{2}}\left[\begin{array}{cc}
1 & 1 \\
-1 & 1
\end{array}\right]
$$

Because of the linear independence of the columns in $\mathbf{G}$, the matrix $\mathbf{G}$ is 
invertible and equation (G.6) takes the form

$$
\mathrm{A}=\mathrm{G}^{-1} \Lambda \mathrm{G}
$$

Placing this relationship for A back into equation (G.5) gives

$$
\mathbf{2 T}=\mathbf{k}^{T} \mathbf{G}^{-1} \Lambda \mathbf{G} \mathbf{k}
$$

This can be taken a step further by making the substitutions $\mathbf{z}^{T}=\mathbf{k}^{T} \mathbf{G}^{-1}$ and $\mathbf{z}=\mathbf{G k}$, now giving

$$
\mathbf{2} \mathbf{T}=\mathbf{z}^{T} \boldsymbol{\Lambda} \mathbf{z}=\lambda_{1} \mathbf{z}_{1}^{2}+\lambda_{2} \mathbf{z}_{2}^{2},
$$

where $\lambda_{1}$ and $\lambda_{2}$ are the eigenvalues. At this point, multiple regression analysis can be carried out to solve for $\mathbf{z}_{1}$ and $\mathbf{z}_{2}$, and then $\mathbf{z}=\mathbf{G k}$ can be solved in order to find the original unknowns.

Equation (G.12) and the eigenvalues can now be used to make the transformation from an expression in terms of $k$ to an expression in terms of $z$. This gives the equation for the linear regression,

$$
2 T=z_{1}^{2}+(\cos 2 \theta) z_{2}^{2}
$$

Multiple regression analysis is particularly effective for components that have an 
orientation-dependent transmission, such as a polarizer. A least-squares fitting routine calculates the unknowns based on the transmission measurements at various component orientations.

After expressing the transmittance equation in the appropriate form for regression analysis, solving for the unknown parameters involves implementing a leastsquares fit of the observations (transmittance measurements, $T$ ) on the regressors (eigenvalues that incorporate transmission angle, 1 and $\cos 2 \theta$ ). This is seen in the following equation,

$$
\left[\begin{array}{c}
2 T_{1} \\
2 T_{2} \\
\vdots \\
2 T_{N}
\end{array}\right]=\left[\begin{array}{cc}
1 & \cos 2 \theta_{1} \\
1 & \cos 2 \theta_{2} \\
\vdots & \vdots \\
1 & \cos 2 \theta_{N}
\end{array}\right]\left[\begin{array}{c}
z_{1}^{2} \\
z_{2}^{2}
\end{array}\right] .
$$

With $\mathbf{G}$ previously established, the transformation between $z$ and $k$ can be made. After solving for $z_{1}^{2}$ and $z_{2}^{2}$ using the least-squares fit and taking the square root, the transformation relationship $\mathbf{z}=\mathbf{G k}$ can be used for expressing $z_{1}$ and $z_{2}$ in terms of $k_{1}^{2}$ and $k_{2}^{2}$,

$$
z_{1}=\frac{k_{1}^{2}+k_{2}^{2}}{\sqrt{2}} \quad \text { and } \quad z_{2}=\frac{k_{1}^{2}-k_{2}^{2}}{\sqrt{2}}
$$

Rearranging provides the relationship between the least-squares fit results $\left(z_{1}\right.$ and $\left.z_{2}\right)$ 
and the unknowns $\left(k_{1}^{2}\right.$ and $\left.k_{2}^{2}\right)$,

$$
k_{1}^{2}=\frac{\sqrt{2}}{2}\left(z_{1}+z_{2}\right) \quad \text { and } \quad k_{2}^{2}=\frac{\sqrt{2}}{2}\left(z_{1}-z_{2}\right) .
$$

The least-squares fit routine produced estimates for $z_{1}^{2}$ and $z_{2}^{2}$, so the square root of these two parameters must be taken to solve for the $k$ values. This results in a positive and negative root that require a specific combination in order to get the correct results. Guidelines for determining the specific combination are that $k_{1}^{2}$ is expected to be close to unity and $k_{2}^{2}$ is expected to be a small, nonzero value. 


\section{Appendix H Incidence angle effects}

The Stokes/Mueller formalism typically assumes normal illumination, which is not the case with the current measurement system. Because the system is in an imaging configuration with an objective lens, the sample plane is illuminated with a cone of light. This type of illumination increases the pathlength through the sample. Thus, when measuring birefringent samples, a correction factor compensating for the extra pathlength must be included in the sample model. The correction factor modifies the pathlength by using an effective illumination angle. The ensuing section describes incidence angle effects in terms of the numerical aperture of the illuminating lens, and this parameter is used for estimating an effective illumination angle.

\section{Numerical aperture}

The numerical aperture (NA) of a lens describes the range of angles over which it can deliver or collect light. Thus, the NA fixes the solid angle of illumination, and the maximum angle of incidence. This effect is illustrated in Fig. H.1a, as NA and 
incidence angle are related by the expression

$$
\mathrm{NA}=n \sin \psi,
$$

where $n$ is the refractive index of the medium in which the lens is operating and $\alpha$ is the maximum acceptance angle. For the current system, $n=1$ for air and NA = 0.28 , resulting in a maximum acceptance angle of $\psi=16.26^{\circ}$.

The solid angle subtended by the lens, as viewed from the sample plane (see Fig. H.1b), is

$$
\Omega=\int_{0}^{2 \pi} d \phi \int_{0}^{\psi} \sin \theta d \theta=2 \pi\left(1-\sqrt{1-\mathrm{NA}^{2}}\right),
$$

where $\theta$ is the zenith angle and $\phi$ is the azimuth angle. A numerical aperture of $\mathrm{NA}=0.28$ gives a solid angle of $\Omega=0.251$ radians.

Each illumination angle within the illumination cone contributes a specific amount of light to the sample, which is described by the corresponding solid angle. In other words, for a specific angular window between $\psi_{1}$ and $\psi_{2}$ (see Fig. H.2), the

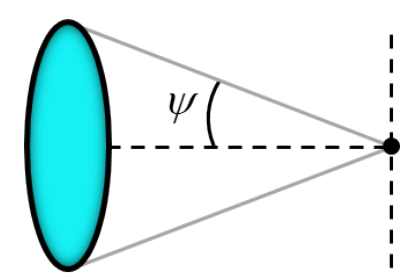

(a)

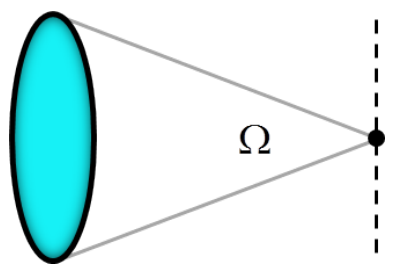

(b)

Figure H.1: Schematic illustrating a) the maximum incidence angle of a lens, b) the solid angle subtended by a lens. 


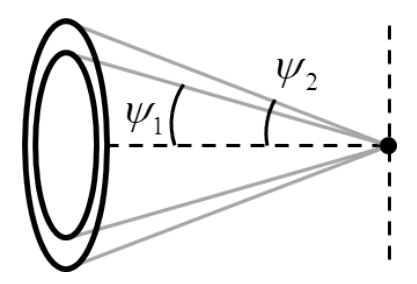

Figure H.2: Geometry for calculating the solid angle between two angles $\psi_{1}$ and $\psi_{2}$.

solid angle can be calculated from

$$
\Omega_{\text {window }}=\int_{0}^{2 \pi} d \phi \int_{\psi_{1}}^{\psi_{2}} \sin \theta d \theta=2 \pi\left(\cos \psi_{1}-\cos \psi_{2}\right) .
$$

For example, the solid angle for the angular window of $\psi_{1}=0^{\circ}$ to $\psi_{2}=1^{\circ}$ is $\Omega_{\text {window }}=9.57 \times 10^{-4}$ radians. As another example, the solid angle for the window of $\psi_{1}=15.26^{\circ}$ to $\psi_{2}=16.26^{\circ}$ is $\Omega_{\text {window }}=0.030$ radians. These two calculations show that the inner degree of illumination $\left(\psi=0^{\circ}-1^{\circ}\right)$ contributes $0.38 \%$ to the overall solid angle of illumination, while the outer degree $\left(\psi=15.26^{\circ}-16.26^{\circ}\right)$ contributes 11.9\%. In other words, the edge rays contribute more than the central rays. Thus, it is necessary to calculate a mean solid angle for representing the illumination cone, from which an effective illumination angle can be computed. The mean solid angle is $\bar{\Omega}=0.084$ radians, which corresponds to an effective illumination angle of $\psi_{\text {eff }}=9.38^{\circ}$.

\section{Pathlength modification}

Figure H.3 shows a schematic of the lens illuminating a sample of thickness $t$. In this schematic, the lens focuses the cone of light to a region in the middle of the sample. 
The maximum pathlength $p$ (the red line in Fig. H.3) a ray of light travels to reach the center of the sample is

$$
p=\frac{t}{2 \cos \psi},
$$

and the total distance through the sample is

$$
p_{\text {total }}=\frac{t}{\cos \psi} .
$$

Now consider a birefringent medium of thickness $t$. This type of medium causes a relative phase delay between the orthogonal components of the incident field. The phase delay, or retardance $\delta$, is a function of thickness. Thus, when the birefringent sample is illuminated with a cone of light, the retardance now includes the cosine term to compensate for the longer pathlength, and the angle is the effective illumination angle $\psi_{\text {eff }}$

$$
\delta=\frac{2 \pi t \Delta n}{\lambda \cos \psi_{\mathrm{eff}}},
$$

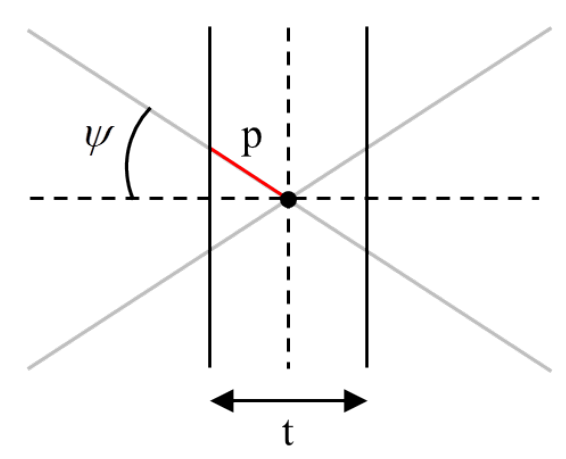

Figure H.3: Schematic illustrating the maximum pathlength a ray of light travels through a sample of thickness $t$. 
where $\Delta n$ is the birefringence and $\lambda$ is the illumination wavelength. This correction factor will be used for modeling the effects from subsequent measurements on birefringent media, such as wave plates and rock samples containing birefringent minerals. 